\author{
Universidade De SÃo PaUlo \\ Instituto de Física de SÃo CARlos \\ Departamento de Física TeÓRICA
}

\title{
Aglomerados Abertos: Determinação de Parâmetros Cinemáticos e Fundamentais
}

\author{
Victória Flório Pires de Andrade
}

Dissertação apresentada ao Instituto de Física de São Carlos, Universidade de São Paulo, para obtenção do Título de Mestre em Ciências: Física Básica

Área de Concentração:Astronomia ORIEntador:Prof. Dr. Wilton da Silva Dias

SÃo CARLOS 
$|F S C-S B|$

CLASS

CUTTER

TOMBO.. I. I..........

Andrade, Victória Flório Pires de

Aglomerados abertos: determinação de parâmetros cinemáticos e fundamentais / Victória Flório Pires de; orientador Wilson da Silva Dias.- São Carlos, 2007.

$108 \mathrm{p}$.

Dissertação (Mestrado em Física Básica - Área de concentração: Astronomia) - Instituto de Física de Sảo Carlos da Universidade de Säo Paulo.

1. Aglomerados abertos. 2. Movimentos próprios e probabilídades de pertinência. 3. Paråmetros fundamentais. I. Título. 
MEMBROS DA COMISSÃO JULGADORA DA DISSERTAÇĀO DE MESTRADO DE VICTÓRIA FLÓRIO PIRES DE ANDRADE APRESENTADA AO INSTITUTO DE FISICA DE SÃO CARLOS, UNIVERSIDADE DE SÃO PAULO, EM 27/04/2007.

COMISSÃO JULGADORA:

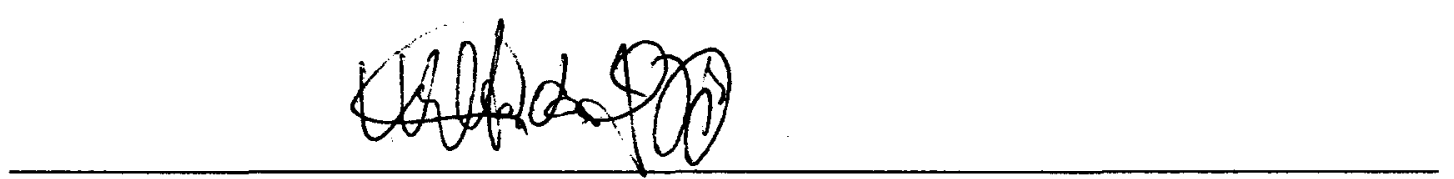

Prof(a). Dr(a). Wilton da Silva Rias (Orientador e Presidente) - UNIFEI

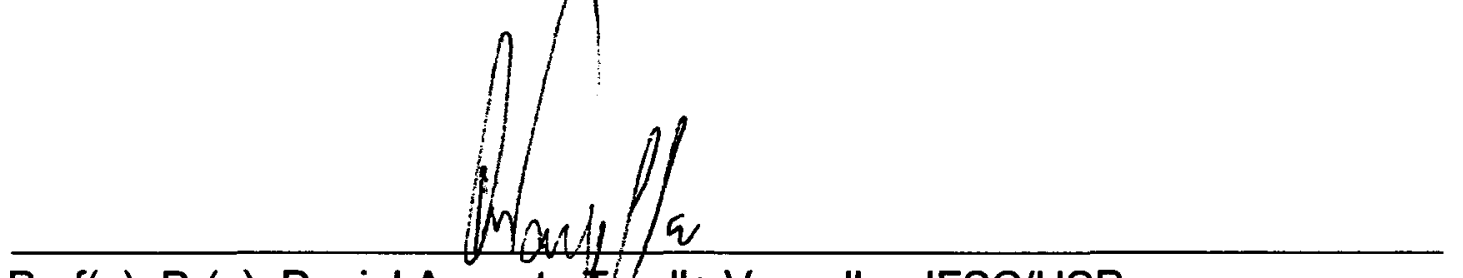

Prof(a). Dr(a). Daniel Augusto /urolla Vanzella - IFSC/USP

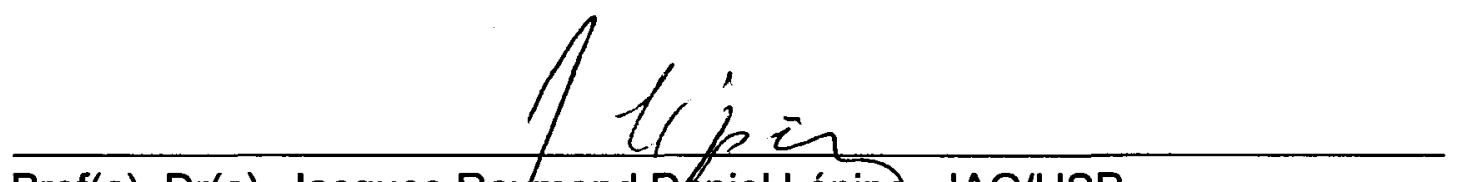

Prof(a). Dr(a). Jacques Raymond Daniel Lépine- IAG/USP 
Aos meus avós, especialmente à vó Ruth e ao vô Mário. 
Agradeço o apoio da Fundação de Amparo à Pesquisa do Estado de São Paulo (FAPESP). 


\section{Agradecimentos}

Este trabalho não teria se realizado se não tivessem participado dele comigo minha família e amigos. Família: Maurício, Vera, Cidinha, Júlia, Laurinha, minha madrinha Valéria que durante a graduação preparava malinhas de guloseimas pra eu trazer pra São Carlos, tia Silvana e tio Marquim que preparam pizzas incríveis, Ícaro e meus quatro avós. Ao vô Mário especialmente, que não sabia direito o que eu fazia em São Carlos, mas de seu jeito sempre perguntava se eu 'tinha precisão de alguma coisa', que saudades sinto dele! Meus amigos também, são tão importantes em minha vida.. são tantos e tão queridos, não sei o que seria de mim sem eles! Agradeço à minha mãe, pai e tia Cida em especial, que durante suas vidas lutaram para que eu e minhas irmãs tivéssemos uma boa educação, isso para mim significa muito. Também agradeço em especial à minha irmã Laura (Lupin), e à Júlia (Booo - hehe). Agradeço aos meus parentes e bons amigos aqui em São Carlos : Estela e João, que sempre me acolheram e ajudaram em diversos momentos.

Tenho muito que agradecer ao professor Wilton da Silva Dias e sua esposa Bete, e ao professor Válter Líbero. Ao Wilton agradeço por ter me aceitado como orientanda, por ter me confiado seu projeto, por ter me acompanhado nas dificuldades de uma orientação à distância e porque durante sua estada em São Carlos foi um bom amigo. Ao professor Válter Luiz Líbero devo muito, por ter me me recebido como orientanda perante à FAPESP sem exitar, num determinado período do projeto. Agradeço à ele por ter me recebido sempre com extremas simpatia, cordialidade e presteza, por ter aceitado nosso projeto como se fosse seu, em termos de cuidado e atenção além de ter revisado comigo o texto da dissertação. Devo expressar minha profunda admiração pela profissão de professor, e na figura desses dois agradeço a todos aqueles que participaram de minha formação desde o princípio.

Não posso deixar de citar de forma alguma o grande amigo Arthur Gustavo, que ajudou com as ferramentas computacionais, foi sempre prestativo com minhas dúvidas infernais na área de programação e por ter desenvolvido o programa Stax, tão útil no dia a dia do meu trabalho. 
Ao amigo Fabiano (God Tex) agradeço por ter me passado seus conhecimentos sobre o latex, ferramenta com a qual escrevi o texto da dissertação. Também agradeço ao solidário amigo Daniel por me emprestar sua dissertação de mestrado para dar uma olhadela, muito embora fosse na área de Teoria do Funcional Densidade (hehehe).

Não me esqueço das companheiras de república (Ana Carla (belezinha), Marcelle, Suelene, Cris e Sami), dos grandes amigos e vizinhos da família Solar dos Engenheiros: P.H., Eric, Alessandro, Morgado, Tica, Bachur, Ingrid, Júlia, Presidente, João Vítor, Castanha, Flash, Garçom, Japonês (afilhado), Thiagão e Guu..., do pessoal do corredor da física teórica aqui do IFSC : os Fávaro Ana Paula e Guiga, Marcos Felipe..., e meus companheiros de senzala: Cassiii, Poliii e Blaniii, do pessoal do prédio da teoria: Bruninha(amigona), Ednei..., do pessoal que trabalha na biblioteca (as meninas da biblioteca são sempre simpáticas e dispostas a ajudar: Betânia, Lú, Mara, Neuza, Paula, Jú e Edileuza), do pessoal da portaria : seu Zé, Elisa e D. Idalina, Ioná..., da Cristina Ligo secretária do grupo de teoria, sempre me ouvindo, ajudando a resolver os pepinos, dando idéias..., dos amigos do prédio do labirinto: Edmir (afilhado), Pipa, Daniel, Jorge (el mexicano famoso), Jonathas (Fujão), Luís, Dri, Robin, Rivas, Polina..., aos companheiros de mestrado, CAFÉ, discussões..., à Vaninha da cantina, ao pessoal que trabalha na Comissão de Pós-Graduação por seu atendimento : Wladerez e Sílvio, agradeço muito também a Edvane da graduação.

Aos grandes amigos Paulo Eduardo, que está se divertindo muito com Teoria de Campos em Londres, João Vítor que está se divertindo muito como professor de física e Afonso, que realmente está se divertindo muito, estudando a mente dos físicos na psicologia. Um muito obrigada especial vai a todos os meus colegas de graduação em física, não pude citá-los todos, apesar de ainda continuar no convívio de muitos deles, são pessoas muito especiais de quem vou me lembrar para sempre! Agradeço aos grandes amigos da cidade natal de Catalão : Tanhonha, Rosa, Aldão, Karencita, Marden, Chica, Joyce, Tasso (trufas), Faustim, Andréia, Saura, Sabrina, Isabel (árvore), Alessandra (aia boa), Lívia, Fred's, Paulista... são muitos, àqueles que organizaram uma vaquinha pra me presentear com a assinatura da Super Interessante num passado remoto, obrigada a todos. Agradeço também às minhas colegas de iniciação científica Barb's e Perescíola, foi bom nosso convívio e sei que nossa amizade vai perdurar. 
Obrigada à FAPESP pelo apoio financeiro durante o mestrado, e ao IFSC por ter sido sede da realização do sonho de me tornar uma bacharel em física. São muitas as coisas que nos levam a ser quem somos, são muitas as pessoas que participam de nossas vidas todos os dias, que nos estimulam, que nom cumprimentam, que passam por nós, que nos fazem sorrir e nos sorriem também, cada uma tem sua importância, por isso são muitas as reticências necessárias em meus agradecimentos, porque são muitas as pessoas que desempenharam um papel especial no meu caminhar por entre a gente. 


\section{Resumo}

Nosso grupo tem dedicado atenção especial em manter o Novo Catálogo de Aglomerados Abertos e Candidatos Opticamente Visíveis (Dias et.al. [1] - denominado comumente DAML02), que vem sendo continuamente atualizado com novos resultados vindos da literatura. Além de manter o catálogo, nosso grupo está constantemente produzindo novos resultados como movimentos próprios médios e determinação de probabilidades de pertinência de estrelas aos aglomerados estudados (Dias et. al. [2] [3] [4]), também descobrindo novos aglomerados abertos (Alessi et. al. [5]) contribuindo para o conhecimento da amostra de aglomerados abertos conhecida na nossa Galáxia.

Neste trabalho nós apresentamos os primeiros resultados de um estudo cinemático e fotométrico de uma amostra de aglomerados abertos com distância e idade desconhecidas. Trata-se de uma amostra de 850 aglomerados originalmente listados na versão 2.3 do catálogo DAML02. As probabilidades de pertinência das estrelas na região de cada aglomerado foram determinadas através de métodos estatísticos conhecidamente eficazes como (Vasilevskis e Rach [6], Sanders [7] e Zhao e He [8] aplicados aos movimentos próprios individuais UCAC2 (Zacharias et.al.[9]). Consequentemente, os movimentos próprios médios dos aglomerados também foram estimados. Utilizando apenas as estrelas membro e dados fotométricos 2MASS (Skrutskie et.al.[10]) construimos os diagramas cor-magnitude para cada aglomerado que nos permitiram estimar parâmetros fundamentais dos aglomerados como distâncias, excessos de cor nas bandas do infravermelho e idades. A determinação desses parâmetros envolve o ajuste de isócronas a sequência principal do aglomerado e a partir de valores iniciais ajustamos essas curvas para a obtenção dos valores de distâncias, excessos de cor e idades. Desenvolvemos com o objetivo de fornecer esses valores preliminares, um programa que usa apenas as estrelas com Tipo Espectral identificado no SIMBAD.

Portanto, neste trabalho determinamos movimentos próprios e probabilidades de pertinência para 319 aglomerados. Desses, 32 apresentaram solução para os parâmetros fundamentais (distância, excesso de cor e idade) e segundo nossas análises para os casos inéditos, 11 aglomerados encontram-se na vizinhança solar. Os erros envolvidos estimados são totalmente coerentes com os erros do catálogo DAML02.

Palavras chave: 1. Aglomerados abertos. 2. Movimentos próprios e probabilidades de pertinência. 3. Parâmetros fundamentais. 


\begin{abstract}
Our group has dedicate a special attention to maintain the New Catalogue of Optically Visible Open Clusters and Candidates (Dias et al.[1] - hereinafter DAML02) that is being continuously updated with new results from the literature. Besides maintaining the catalogue, our group is actively producing new results such as the mean absolute proper motion and membership determination (Dias et. al. [2] [3] [4]) and discovering new open clusters (Alessi et al.[5]) contributing to complete the knowledge of the hole sample of open clusters in the Galaxy.

In this work we present the first results of the kinematic and photometric study of the open clusters with unknown distance and age in DAML02. The membership probablities of the stars in the region of each cluster were determined applying the statistical method of Zhao e He [8] using the individual stellar UCAC2 (Zacharias et.al.[9]) proper motions. Consequently, the mean absolute proper motion of the clusters were also estimated. Using the investigated stars we were able to construct the colourmagnitude diagrams that allowed us to derive preliminary estimates of the fundamental parameters of the clusters such as reddening, distance and ages where the 2MASS
\end{abstract} (Strutskie et.al.[10]) photometric data were considered.

Keywords: 1. Open clusters. 2. Proper motion and membership. 3. Fundamental parameters. 


\section{Sumário}

1 Introdução 1

1.1 Os Aglomerados Abertos e seu papel na Astronomia . . . . . . . . . . . 1

1.2 Aglomerados Abertos e Perspectivas Históricas . . . . . . . . . . . 4

1.3 Catálogos de Aglomerados Abertos e o Catálogo DAML02 . . . . . . 5

2 Movimentos Próprios e probabilidades de pertinência 10

2.1 Determinação de Movimentos Próprios e probabilidades de pertinência: A evolução do Método . . . . . . . . . . . . . . . . . . . . 10

2.2 O Método para o Cálculo de Movimentos Próprios e probabilidades de pertinência . . . . . . . . . . . . . . . . . . 12

2.3 Dados utilizados e análise dos resultados fornecidos pelo programa . . . 17

2.4 Confiabilidade do Método . . . . . . . . . . . . . . . . 19

3 Parâmetros Fundamentais $\quad 24$

3.1 Distâncias e Idades . . . . . . . . . . . . . . . . . . . . . 24

3.2 O projeto $2 M A S S$ e as Isócronas de Padova . . . . . . . . . . . . . 27

3.3 Cálculo de distâncias e idades : O Método . . . . . . . . . . . 28

4 Resultados - Parâmetros Cinemáticos $\quad 31$

4.1 Resultados de Movimentos Próprios e probabilidades de pertinência para os aglomerados estudados . . . . . . . . . . . . . . . . 31

4.1.1 Comparações com resultados obtidos na literatura . . . . . . . . 33

4.1.2 Comparação com os resultados disponíveis no DAML02 . . . . . 33

4.1.3 Comparação com os resultados fornecidos por Kharchenko et. al.

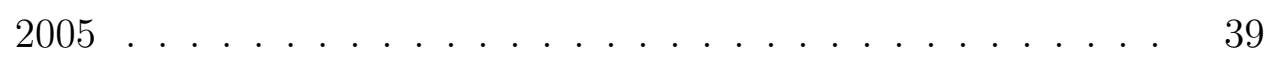

4.1.4 Casos sem solução . . . . . . . . . . . . . . . . . . 41 
5 Resultados - Parâmetros Fundamentais

5.1 Resultados de Parâmetros Fundamentais para os aglomerados estudados 45

5.1.1 Casos que apresentam resultados catalogados . . . . . . . . 46

5.1 .2 Soluções Inéditas . . . . . . . . . . . . . . . . 50

5.2 Estimativa de erros para os valores de distância, idades e excessos de cor 54

5.3 Valores iniciais fornecidos para o ajuste das isócronas . . . . . . . 61

5.4 Casos de difícil solução . . . . . . . . . . . . . . . . . . 64

6 Conclusões e Perspectivas $\quad 67$

7 Apêndices $\quad \mathbf{7 0}$

7.1 Diagramas CMD dos aglomerados estudados . . . . . . . . . . 72

7.1.1 Casos estudados no projeto . . . . . . . . . . . 73

7.1.2 Casos em comum com a amostra de Pauzen e Netopil . . . . . . 73

8 Tabelas $\quad 84$

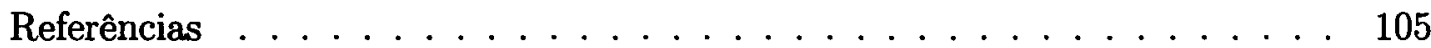




\section{Lista de Figuras}

1.1 A figura traz uma representação da Galáxia, mostrando regiões como o halo, o disco e o núcleo galáctico. Os aglomerados abertos se localizam no disco galáctico enquanto que os globulares no halo. Também é apontada a posição do Sol na Galáxia. Considera-se que a Galáxia possua em toda sua extensão aproximadamente $50 \mathrm{Kpc}$. A imagem foi extraída na página disponível em http://casswww.ucsd.edu/public/tutorial/MW.html 23/02/2007. . . . . . . . . . . . . . . . .

1.2 A figura traz fotos dos aglomerados M5 (aglomerado globular) e NGC 4755 - Caixa de Jóias (aglomerado aberto). Como se pode ver, nos aglomerados globulares as estrelas possuem uma localização angular aparente bem próxima, diferente dos aglomerados abertos. . . . . . . . . . .

1.3 No diagrama são ilustrados os principais braços (Perseus, local e SagitariusCarina) obtidos a partir da localização de aglomerados abertos. O sol se situa no centro do diagrama $(x=0, y=0)$. São utilizados cerca de 109 aglomerdos abertos com idades inferiores a 10 milhões de anos. As coordenadas equatoriais dos aglomerados foram convertidas para coordenadas galácticas e depois para x,y galácticas considerando a distância. 
2.1 Diagramas VPD (à esquerda) e CMD (à direita) para o aglomerado Skiff $J 0619+18.5$, ferramentas de análise do programa utilizado para o cálculo de movimentos próprios médios dos aglomerados. Em vermelho as estrelas com probabilidades de pertinência maiores que 51\%. O diagrama VPD mostra pontos referentes aos valores de movimentos próprios das estrelas nas duas coordenadas $\mu \alpha \cos \delta$ e $\mu \delta$ em mas/yr, enaquanto que o diagrama CMD mostra os pontos referentes a magnitude das estrelas nas bandas J-H X J do infravermelho próximo. . . . . . . . . . . .

2.2 Diagrama de probabilidades de pertinência das estrelas ao aglomerado Skiff J0619+18.5. Uma das ferramentas mais importantes na análise dos dados de saída do programa, o diagrama mostra as probabilidades de pertinência das estrelas do campo ao aglomerado. . . . . . . . . . . .

2.3 Comparação dos nossos resultados para movimentos próprios médios com aqueles fornecidos por Baumgardt et. al. (2000); Dias et. al. (2001,2002); Beshenov e Loktin(2004), Kharchenko et. al. (2003)(BDW, DLA, BL, KPP, respectivamente). As linhas de $45^{\circ}$ representam movimentos próprios iguais. À direita temos a distribuição das diferenças em movimentos próprios para cada comparação, levando em consideração componentes de ascenção reta e declinação pesados pelos erros formais (equação 2.10); eles indicam que não há distinção entre os movimentos próprios comparados dentro dos erros estimados. . . . . . . . . . . .

2.4 Diferenças em movimento próprio médio absoluto (UCAC2-Dias et. al. 2001, 2002)[2] em $\mu \alpha \cos \delta$ e $\mu \delta$. Os círculos mostram as regiões de desvio padrão correspondentes a um, dois e três $\sigma$ obtidas neste estudo. . . .

4.1 Apresentamos a comparação dos movimentos próprios médios obtidos neste trabalho na coordenada $\mu \alpha \cos \delta$ com outros disponíveis na literatura compilados no nosso catálogo de aglomerados abertos (DAML02). No painel à esquerda não temos um ajuste, mas sim a reta de $45^{\circ}$ que representa regiões de movimento próprio idêntico. O painel à direita representa a distribuição das diferenças em movimentos próprios médios e os símbolos $\langle\Delta\rangle$ e $\sigma$ denotam respectivamente o valor médio das diferenças na coordenada $\mu \alpha \cos \delta$ e o valor do desvio padrão. . . . . . . 
4.2 Apresentamos a comparação dos movimentos próprios médios obtidos neste trabalho na coordenada $\mu \delta$ com outros disponíveis na literatura compilados no nosso catálogo de aglomerados abertos (DAML02). No painel à esquerda não temos um ajuste, mas sim a reta de $45^{\circ}$ que representa regiões de movimento próprio idêntico. O painel à direita representa a distribuição das diferenças em movimentos próprios médios e os símbolos $\langle\Delta\rangle$ e $\sigma$ denotam respectivamente o valor médio das diferenças na coordenada $\mu \delta$ e o valor do desvio padrão. . . . . . . . . .

4.3 O gráfico apresenta diferenças em movimento próprio médio nas coordenadas $\mu \alpha \cos \delta$ e $\mu \delta$. Os círculos mostram as regiões de desvio padrão nas coordenadas, sendo iguais a 1, 2 e 3 vezes o valor de $\sigma$. Alguns dos aglomerados fora do raio de $3 \sigma$ são discutidos no texto. . . . . . . . .

4.4 Diagramas VPD e de probabilidades para os aglomerados Alessi-Teutsch 9, Alessi 22 e Loden 46. Percebemos em todos os casos a existência de muitas estrelas no campo de observação. Consideramos como membros as estrelas que possuem uma probabilidade de pertinência maior que 51\%. 37

4.5 Diagramas VPD para os aglomerados NGC 6738 e Collinder 333, e diagramas de probabilidades de pertinência para as estrelas do aglomerado. Os diagramas mostram a boa separação entre a população de membros e intrusas no campo do aglomerado. Lembramos que consideramos como membros as estrelas que possuem uma probabilidade de pertinência maior que 51\%, no diagrama VPD os membros estão representados pela cor vermelha. . . . . . . . . . . . . . . .

4.6 Comparação do movimento próprio médio em $\mu \alpha \cos \delta$ obtidos neste trabalho com os valores fornecidos por K05 compilados no nosso catálogo de aglomerados abertos (DAML02). No painel da esquerda a reta de $45^{\circ}$ representa regiões de movimento próprio idêntico. O painel à direita mostra a distribuição das diferenças em movimentos próprios e os símbolos $\langle\Delta\rangle$ e $\sigma$ denotam respectivamente o valor médio das diferenças na coordenada $\mu \alpha \cos \delta$ e o valor do desvio padrão. . . . . . . . . . . 
4.7 Comparação do movimento próprio na coordenada $\mu \delta$ obtidos neste trabalho com os obtidos por K05. A reta na figura do painel à esquerda não é um ajuste, a reta de $45^{\circ}$ representa regiões de movimento próprio idêntico. No painel à direita temos a distribuição das diferenças em movimentos próprios e os símbolos $\langle\Delta\rangle$ e $\sigma$ denotam respectivamente o valor médio das diferenças na coordenada $\mu \delta$ e o valor do desvio padrão.

4.8 O gráfico apresenta diferenças em movimento próprio médio nas coordenadas $\mu \alpha \cos \delta$ e $\mu \delta$. Os círculos mostram as regiões de desvio padrão nas coordenadas, sendo iguais a 1, 2 e 3 vezes o valor de $\sigma$. Os aglomerados fora do raio de $3 \sigma$ são os mesmo aglomerados dicutidos na comparação inserida na seção 4.1.2. . . . . . . . . . . . . . . . . . . . . .

4.9 Diagramas $V P D$ e $C M D$ para o aglomerado ESO 245-09, ferramenta de análise do programa utilizado para o cálculo de movimentos próprios médios dos aglomerados. Em vermelho as estrelas com probabilidades de pertinência maiores que $50 \%$. . . . . . . . . . . . .

4.10 Imagens tiradas do Digitized Sky Survey dos aglomerados cujos movimentos próprios não puderam ser determinados. Os tamanho das imagens dos aglomerados Herschel 1, ESO 429-13, ESO 493-03, ESO 245-09, Dutra-Bica 5 e ESO 456-72 são respectivamente 43.2, 4, 7, 14, 0.4 e 1.4 todos em minutos de arco. Diâmetros e coordenadas foram extraídos do catálogo DAML02. . . . . . . . . . . . . . . . .

5.1 Diagramas cor-magnitude dos aglomerados Alessi 34, Alessi-Teutsch 3, Loden 189 e Teutsch 35, nas bandas $J-K \times J$ do infravemelho para os aglomerados cujas soluções de parâmetros fundamentais concordam com os resultados do DAML02. Nos diagramas dos aglomerados temos o ajuste das isócronas à sequência principal. Em vermelho e preto as isócronas cujo ajuste foi feito neste trabalho e em verde e rosa as isócronas ajustadas com os valores do DAML02. As isócronas ajustadas possuem metalicidade solar de acordo com os modelos de Padova. . . . . . . . . 
5.2 Diagramas cor-magnitude dos aglomerados Loden 694, Loden 2313 e Loden 807 , nas bandas $J-K \times J$ do infravemelho para os objetos cujas soluções de parâmetros fundamentais discordam em distância e excesso de cor do DAML02. Nos diagramas dos aglomerados temos o ajuste das isócronas à sequência principal. Em vermelho e preto as isócronas cujo ajuste foi feito neste trabalho e em verde e rosa as isócronas ajustadas com os valores do DAML02. Apresentamos também os diagramas de cada aglomerado considerando apenas estrelas membros com raio menor.

5.3 Diagramas cor-magnitude dos aglomerados inéditos Harvard 16 e Skiff $\mathrm{J} 0619+18.5$, nas bandas $J-K \times J$ do infravemelho. Nos diagramas dos aglomerados temos o ajuste das isócronas à sequência principal. Em vermelho e preto as isócronas cujo ajuste foi feito neste trabalho. Apresentamos também os diagramas de cada aglomerado considerando apenas estrelas membros com raio menor. Todas as isócronas ajustadas possuem metalicidade solar de acordo com os modelos de evolução de Padova. .

5.4 Diagramas CMD dos aglomerados Alessi 62, Collinder 62 e Collinder 416, nas bandas $J-K \times J$ do infravemelho para os objetos cujas soluções de parâmetros fundamentais são inéditas. Nos diagramas dos aglomerados temos o ajuste das isócronas à sequência principal. Em vermelho e preto as isócronas cujo ajuste foi feito neste trabalho. Apresentamos também os diagramas de cada aglomerado considerando apenas estrelas membros com raio menor. Todas as isócronas ajustadas possuem metalicidade solar de acordo com os modelos de evolução de Padova. . . . . . . . . .

5.5 Diagramas CMD dos aglomerados, nas bandas $J-K \times J$ do infravemelho para os objetos cujas soluções de parâmetros fundamentais discordam em distância e excesso de cor do DAML02. Nos diagramas dos aglomerados temos o ajuste das isócronas à sequência principal. Em vermelho e preto as isócronas cujo ajuste foi feito neste trabalho. Apresentamos também os diagramas de cada aglomerado considerando apenas estrelas membros com raio menor. Todas as isócronas ajustadas possuem metalicidade solar $(\mathrm{z} 0)$. 
5.6 O gráfico apresenta diferenças em movimento próprio médio nas coordenadas $\mu \alpha \cos \delta$ e $\mu \delta$. Os círculos referem-se as regiões 1,2 e 3 vezes o valor de $\sigma$. Nenhum aglomerado ficou fora da região de $3 \sigma$. . . . . . .

5.7 Na figura estão os gráficos comparativos entre o ajuste e os resultados obtidos por Pauzen e Netopil para 20 aglomerados abertos. Os valores

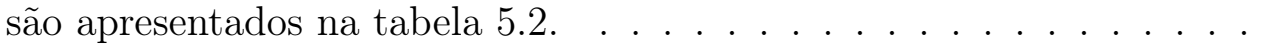

5.8 Diagramas cor-magnitude para os aglomerados Bochum 10 e NGC 6405. As isócronas foram ajustadas à sequência principal dos aglomerados no intuito de determinar distâncias, excessos de cor e idades. Em vermelho e preto as isócronas referentes ao valores do nosso ajuste e em verde e rosa referentes aos valores publicados por Pauzen e Netopil. À direita apresentamos um diagrama cor-magnitude exibindo erros nas bandas do infravermelho $J$ e $H$ extraídos do catálogo 2MASS juntamente com o ajuste dos valores de parâmetros fundamentais do nosso ajuste às isócronas (verde e rosa). Nem todas as estrelas do aglomerado apresentam erros $J$ e $H$ publicados no 2MASS. Todas as isócronas ajustadas possuem metalicidade solar de acordo com os modelos de Padova. . . . . . . .

5.9 Diagramas cor-magnitude para os aglomerados Stock 14 e Trumpler 10. As isócronas foram ajustadas à sequência principal dos aglomerados no intuito de determinar distâncias, excessos de cor e idades. Em vermelho e preto as isócronas referentes ao valores do nosso ajuste e em verde e rosa referentes aos valores publicados por Pauzen e Netopil. À direita apresentamos um diagrama cor-magnitude exibindo erros nas bandas do infravermelho $J$ e $H$ extraídos do catálogo 2MASS juntamente com o ajuste dos valores de parâmetros fundamentais do nossso ajuste às isócronas (verde e rosa). Nem todas as estrelas do aglomerado apresentam erros $J$ e $H$ publicados no 2MASS. Todas as isócronas ajustadas possuem metalicidade solar de acordo com os modelos de Padova. . . . . . . . . 
5.10 Diagramas CMD J-HxJ e J-KxJ, para o aglomerado NGC 1647. Em vermelho as estrelas com probabilidades de pertinência maiores que 50\%, as isócronas ajustadas com nossos valores estão em vermelho e preto, as ajustadas com os valores publicados por Pauzen e Netopil em verde e rosa. Apresentamos também os valores numéricos referentes aos ajustes dos parâmetros fundamentais. As isócronas ajustadas possuem metalicidade solar de acordo com os modelos de Padova. . . . . . . . . . . .

5.11 Comparação da distância, excesso de cor $\mathrm{E}(\mathrm{J}-\mathrm{H})$ e idade dos valores obtidos com o nosso ajuste e DAML02. . . . . . . . . . . .

5.12 Diagramas CMD dos aglomerados NGC 2364, NGC 2306, Dolidze 11 e Loden 153, nas bandas $J-H \times J$ do infravemelho para os objetos cujas soluções de parâmetros fundamentais não foi obtida. . . . . . . . . . .

7.1 O gráfico apresenta diferenças em movimento próprio médio nas coordenadas $\mu \alpha \cos \delta$ e $\mu \delta$. Os círculos mostram as regiões de desvio padrão nas coordenadas, sendo iguais a 1,2 e 3 vezes o valor de $\sigma$. . . . .

7.2 Diagramas CMD dos aglomerados Loden 694, Loden 46, NGC 1896, Collinder 416, Teutsch 35 e Skiff J0619+18.5 nas bandas $J-K$ x $J$ do infravermelho. Nos diagramas dos aglomerados temos o ajuste das isócronas à sequência principal. Em vermelho e preto as isócronas cujo ajuste foi feito neste trabalho e em verde e rosa as isócronas ajustadas com os valores do DAML02 para os casos que possuem valores catalogados na literatura. As isócronas ajustadas possuem metalicidade solar de acordo com os modelos de Padova. . . . . . . . . . . . . . . .

7.3 Diagramas CMD dos aglomerados Loden 2313, Loden 306, Loden 189, Harvard 16, Eso 332-08 e Collinder 62 nas bandas $J-K \times J$ do infravermelho. Nos diagramas dos aglomerados temos o ajuste das isócronas à sequência principal. Em vermelho e preto as isócronas cujo ajuste foi feito neste trabalho e em verde e rosa as isócronas ajustadas com os valores do DAML02 para os casos que possuem valores catalogados na literatura. As isócronas ajustadas possuem metalicidade solar de acordo com os modelos de Padova. . . . . . . . . . . . . . . . . . . . 
7.4 Diagramas CMD dos aglomerados Collinder 121, Alessi-Teutsch 3, Antalova 3, Alessi-Teutsch 8, Alessi 62 e Alessi 37 nas bandas $J-K \times J$ do infravermelho. Nos diagramas dos aglomerados temos o ajuste das isócronas à sequência principal. Em vermelho e preto as isócronas cujo ajuste foi feito neste trabalho e em verde e rosa as isócronas ajustadas com os valores do DAML02 para os casos que possuem valores catalogados na literatura. As isócronas ajustadas possuem metalicidade solar de acordo com os modelos de Padova. . . . . . . . . . . . . . .

7.5 Diagramas CMD dos aglomerados Alessi 34, Alessi 21, NGC 6847, NGC 2448, NGC 2260 e NGC 2252 nas bandas $J-K \times J$ do infravermelho. Nos diagramas dos aglomerados temos o ajuste das isócronas à sequência principal. Em vermelho e preto as isócronas cujo ajuste foi feito neste trabalho e em verde e rosa as isócronas ajustadas com os valores do DAML02 para os casos que possuem valores catalogados na literatura. As isócronas ajustadas possuem metalicidade solar de acordo com os modelos de Padova. . . . . . . . . . . . . . . . . .

7.6 Diagramas CMD dos aglomerados Loden 28, Loden 915, Loden 821, Loden 807 e Loden 481 nas bandas $J-K \times J$ do infravermelho. Nos diagramas dos aglomerados temos o ajuste das isócronas à sequência principal. Em vermelho e preto as isócronas cujo ajuste foi feito neste trabalho e em verde e rosa as isócronas ajustadas com os valores do DAML02 para os casos que possuem valores catalogados na literatura. As isócronas ajustadas possuem metalicidade solar de acordo com os modelos de Padova. . . . . . . . . . . . . . . . . . . . . .

7.7 Diagramas CMD dos aglomerados Loden 624 e Loden 1373 nas bandas $J-K \times J$ do infravermelho. Nos diagramas dos aglomerados temos o ajuste das isócronas à sequência principal. Em vermelho e preto as isócronas cujo ajuste foi feito neste trabalho. As isócronas ajustadas possuem metalicidade solar de acordo com os modelos de Padova. . . 
7.8 Casos em comum com Pauzen - Diagramas CMD dos aglomerados NGC 1245, NGC 1647, NGC 2395, NGC 2477, NGC 2527 e NGC 2682 nas bandas $J-K \times J$ do infravermelho. Nos diagramas dos aglomerados temos o ajuste das isócronas à sequência principal. Em vermelho e preto as isócronas cujo ajuste foi feito neste trabalho e em verde e rosa as isócronas ajustadas com os valores do Pauzen e Netopil[34] para os casos que possuem valores catalogados na literatura. As isócronas ajustadas possuem metalicidade solar de acordo com os modelos de Padova. . . .

7.9 Casos em comum com Pauzen - Diagramas CMD dos aglomerados IC 4756, NGC 5662, NGC 5822, NGC 6025, NGC 6067 e NGC 6087 nas bandas $J-K \times J$ do infravermelho. Nos diagramas dos aglomerados temos o ajuste das isócronas à sequência principal. Em vermelho e preto as isócronas cujo ajuste foi feito neste trabalho e em verde e rosa as isócronas ajustadas com os valores do Pauzen e Netopil[34] para os casos que possuem valores catalogados na literatura. As isócronas ajustadas possuem metalicidade solar de acordo com os modelos de Padova. . . .

7.10 Casos em comum com Pauzen - Diagramas CMD dos aglomerados NGC 6250, NGC 6405, NGC 6834, NGC 6871, Bochum 10 e Collinder 394 nas bandas $J-K \times J$ do infravermelho. Nos diagramas dos aglomerados temos o ajuste das isócronas à sequência principal. Em vermelho e preto as isócronas cujo ajuste foi feito neste trabalho e em verde e rosa as isócronas ajustadas com os valores do Pauzen e Netopil[34] para os casos que possuem valores catalogados na literatura. As isócronas ajustadas possuem metalicidade solar de acordo com os modelos de Padova. . . .

7.11 Casos em comum com Pauzen - Diagramas CMD dos aglomerados Trumpler 10 e Stock 14 nas bandas $J-K$ x $J$ do infravermelho. Nos diagramas dos aglomerados temos o ajuste das isócronas à sequência principal. Em vermelho e preto as isócronas cujo ajuste foi feito neste trabalho e em verde e rosa as isócronas ajustadas com os valores do Pauzen e Netopil[34] para os casos que possuem valores catalogados na literatura. As isócronas ajustadas possuem metalicidade solar de acordo com os modelos de Padova. . . . . . . . . . . . . . . . . . . 


\section{Lista de Tabelas}

5.1 Apresentamos 4 aglomerados cujas soluções estão em bom acordo com os valores da literatura DAML02. Na tabela dados referentes aos valores de distância em parcecs, excesso de cor $E(J-H)$ e $\log (\mathrm{t})$ para o ajuste e para DAML02. . . . . . . . . . . . . . . . .

5.2 Tabela dos valores calculados para média, desvio padrão e erros da diferença entre nossos resultados de parâmetros fundamentais e os obtidos por Pauzen para uma amostra de 20 aglomerados abertos. . . . . . .

5.3 Aglomerados conhecidos utilizados no cálculo de parâmetros fundamentais: Bochum 10, NGC 6405, Stock 14 e Trumpler 10. São fornecidas posições J2000 extraídas do DAML02, sendo $\alpha$ dada em hhmmss e $\delta$ dada em $\left(o^{\prime \prime \prime}\right)$. São dados valores do nossso ajuste para parâmetros fundamentais (distância dada em parcecs, $E(J-H)$ e $\log (\mathrm{t})$ ) e também os resultados publicados por Pauzen [34] para os mesmos aglomerados.

7.1 Objetos com parâmetros conhecidos cujos movimentos próprios foram investigados. Mostramos na tabela a comparação entre os valores obtidos com o nosso ajuste e os publicados em um artigo do grupo [4] para os movimentos próprios dos aglomerados nas duas coordenadas $\mu \alpha \cos \delta$ e $\mu \delta$ em mas/yr. O erro mostrado é calculado de acordo com a relação: erro = $\frac{\sigma}{\sqrt{n_{c}-1}}, N_{c}$ e $N_{a}$ denotam o número de estrelas membro consideradas em nossa análise e no artigo respectivamente. . . . . . . . . . . . 
8.1 Tabela A - Resultados das análises de movimentos próprios, onde $N_{e}$ é o número de estrelas do aglomerado, $N_{i}$ o número de estrelas intrusas ao aglomerado, $\mu \alpha \cos \delta$ e $\mu \delta$ são as componentes do movimento próprio em milisegundos de arco, $\sigma$ é a dispersão do movimento próprio das estrelas do aglomerado (UCAC2), $\sigma_{\mu \alpha \cos \delta}$ e $\sigma_{\mu \delta}$ são a dispersão das componentes de movimento próprio das estrelas de campo (DAML02). . . . . . . .

8.2 Tabela B - Comparação entre os resultados UCAC2 e resultados extraídos da literatura DAML02. $N_{e}$ é o número de estrelas do aglomerado, $N_{d}$ o número de estrelas do aglomerado catalogadas no DAML02, $\mu \alpha \cos \delta$ e $\mu \delta$ são as componentes do movimento próprio em milisegundos de arco, $\sigma$ é a dispersão do movimento próprio das estrelas do aglomerado (UCAC2), $\sigma_{\mu \alpha \cos \delta}$ e $\sigma_{\mu \delta}$ são a dispersão das componentes de movimento próprio das estrelas de campo (DAML02).

8.3 Tabela C - Valores dos parâmetros fundamentais (distâncias, excessos de cor nas bandas do infravermelho e idades) para o nosso ajuste e os valores publicados por Pauzen e Netopil. . . . . . . . . . . . .

8.4 Tabela D - Tabela dos 11 aglomerados conhecidos utilizados no cálculo de parâmetros fundamentais. São listadas algumas das estrelas extraídas do SIMBAD utilizadas nos cálculos e suas respectivas probabilidades de pertinência, bem como os ST de cada uma delas. Também são apresentados os resultados de saída do programa Stax; $E(J-H)$ e d são o excesso de cor, obtidos a partir do cálculo no infravermelho; e $E(J-H)$ e d também obtidos a partir do cálculo no visível. Por fim os resultados para $E(J-H)$ e d obtidos no ajuste junto aos diagramas cor-magnitude. 96 
8.5 Tabela E - Apresentamos o nome das estrelas e seus ST's extraídos do SIMBAD bem como suas probabilidades de pertinência fornecidas através do Método descrito na seção 2.2 para o cálculo de movimentos próprios. As estrelas foram usadas nos cálculos dos valores iniciais de distâncias e excessos de cor feitos pelo programa Stax. Os parâmetros fundamentais estão dispostos nas colunas, sendo que exibimos distância, excesso de cor nas bandas do infravermelho $\mathrm{E}(\mathrm{J}-\mathrm{H})$. Nas colunas temos resultados de saída do programa, obtidos a partir da nossa análise, vide seção 3.3 e por fim os valores catalogados no DAML02. Os valores originalmente obtidos do DAML02 para excessos de cor estão nas bandas do visível, aqui utilizamos a relação de conversão: $\frac{E(J-H)}{E(J-K)}=0,309$. Os símbolos "*"indicam os aglomerados para os quais obtivemos como resultados de saída do programa apenas o excesso de cor $\mathrm{E}(\mathrm{J}-\mathrm{H})$ calculado nas bandas do visível ou $\mathrm{E}(\mathrm{J}-\mathrm{H})$ calculado nas bandas do infravermelho, respectivamente para Alessi-Teutsch 8 e Loden 28, conforme foi explanado na seção $3.3 . \ldots \ldots \ldots \ldots \ldots \ldots \ldots$

8.6 Tabela F - Tabela dos 11 aglomerados inéditos cuja solução de parâmetros fundamentais foi obtida neste trabalho. Fornecemos posições (J2000), onde é dada em (hh mm ss - hora minuto segundo) e ( - grau minuto segundo). São listadas algumas das estrelas extraídas do SIMBAD utilizadas nos cálculos de valores preliminares e suas respectivas probabilidades de pertinência, bem como os ST de cada uma delas. E(J$\mathrm{H}$ ), d e logt denotam os resultados obtidos no ajuste junto aos diagramas cor-magnitude para os parâmetros fundamentais. . . . . . . . . . . . . 104 


\section{Capítulo 1}

\section{Introdução}

\subsection{Os Aglomerados Abertos e seu papel na Astrono- mia}

Existem dois tipos de aglomerados: os abertos (ou estelares) e os globulares. Os aglomerados abertos, como o próprio nome sugere, são em geral objetos de fácil resolução com o uso de um telescópio pequeno ou médio, ou seja, as estrelas que os compõe podem ser resolvidas indivualmente. Eles se situam no plano galáctico, e por isso podem também ser denominados de aglomerados galácticos, diferentemente dos aglomerados globulares que se situam no halo galáctico. A figura 1.1 traz um mapa da Galáxia indicando o disco e o halo galáctico, regiões que contém os aglomerados abertos e globulares respectivamente. As diferenças entre aglomerados abertos e globulares se relacionam aos objetos que os compões, sendo que os aglomerados abertos possuem estrelas jovens e quentes, enquanto que os globulares apresentam estrelas frias e velhas.

Os aglomerados abertos e globulares podem ser diferenciados visualmente, os primeiros apresentam estrelas afastadas umas das outras enquanto que nos aglomerados globulares as estrelas estão próximas como mostra a figura 1.2. 


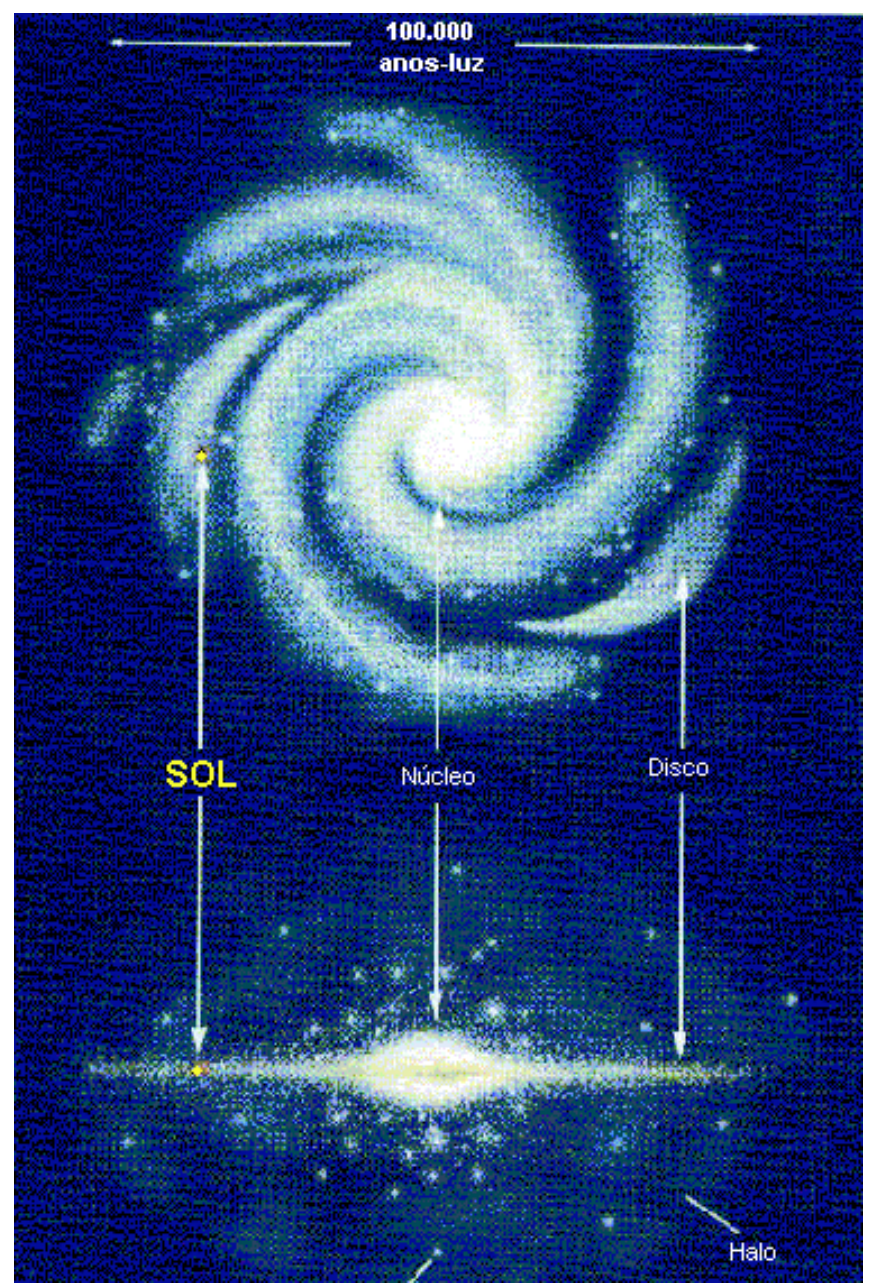

Figura 1.1: A figura traz uma representação da Galáxia, mostrando regiões como o halo, o disco e o núcleo galáctico. Os aglomerados abertos se localizam no disco galáctico enquanto que os globulares no halo. Também é apontada a posição do Sol na Galáxia. Considera-se que a Galáxia possua em toda sua extensão aproximadamente 50 Kpc. A imagem foi extraída na página disponível em http://casswww.ucsd.edu/public/tutorial/MW.html 23/02/2007.

Através do uso de telescópios de alta resolução, chapas fotográficas ou catálogos, podemos distinguir uma região candidata do céu a conter esses aglomerados, sendo que eles podem ser detectados através de um aumento da densidade de estrelas na região observada. Uma região que contém um aglomerado aberto é caracterizada por apresentar dezenas a cerca de um milhar de estrelas que podem ser consideradas a uma mesma distância de nós.

Há um importante fato a respeito dos aglomerados abertos no tocante a formação de seus membros. Acreditamos que todas as estrelas membro de um aglomerado 

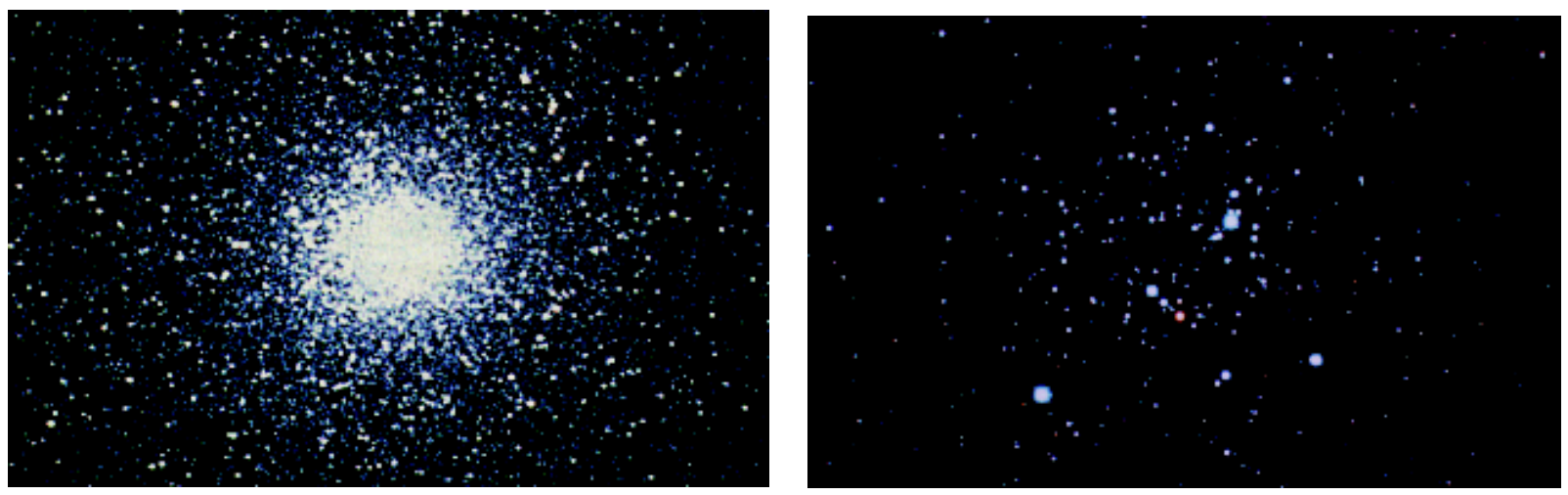

Figura 1.2: A figura traz fotos dos aglomerados M5 (aglomerado globular) e NGC 4755 - Caixa de Jóias (aglomerado aberto). Como se pode ver, nos aglomerados globulares as estrelas possuem uma localização angular aparente bem próxima, diferente dos aglomerados abertos.

aberto tenham se formado juntas, na mesma região do meio interestelar, originárias da mesma nuvem de gás, desse modo possuem também uma mesma composição química e uma mesma idade.

Dessa maneira, o papel dos aglomerados abertos em astronomia é crucial, uma vez que podem ser usados como traçadores eficientes da estrutura espiral da Galáxia, o que tem sido feito há algumas décadas. No entanto, há uma série de perguntas referentes à estrutura espiral que ainda não foram respondidas de forma satisfatória, como por exemplo, a localização dos principais braços, a dependência da taxa de formação estelar nos braços em função da distância ao centro, etc. Uma outra questão re-discutida se refere à existência de um braço espiral na região onde o Sol está localizado [[11],[12]]. A determinação da localização do raio de corrotação também é abordada em trabalhos recentes [[13],[14]] e ainda requer estudos detalhados e principalmente que utilizem maior quantidade de dados cinemáticos.

Na figura 1.3 temos um diagrama extraído do trabalho de Dias [14] que ilustra a importância dos aglomerados abertos como estruturas traçadoras. 


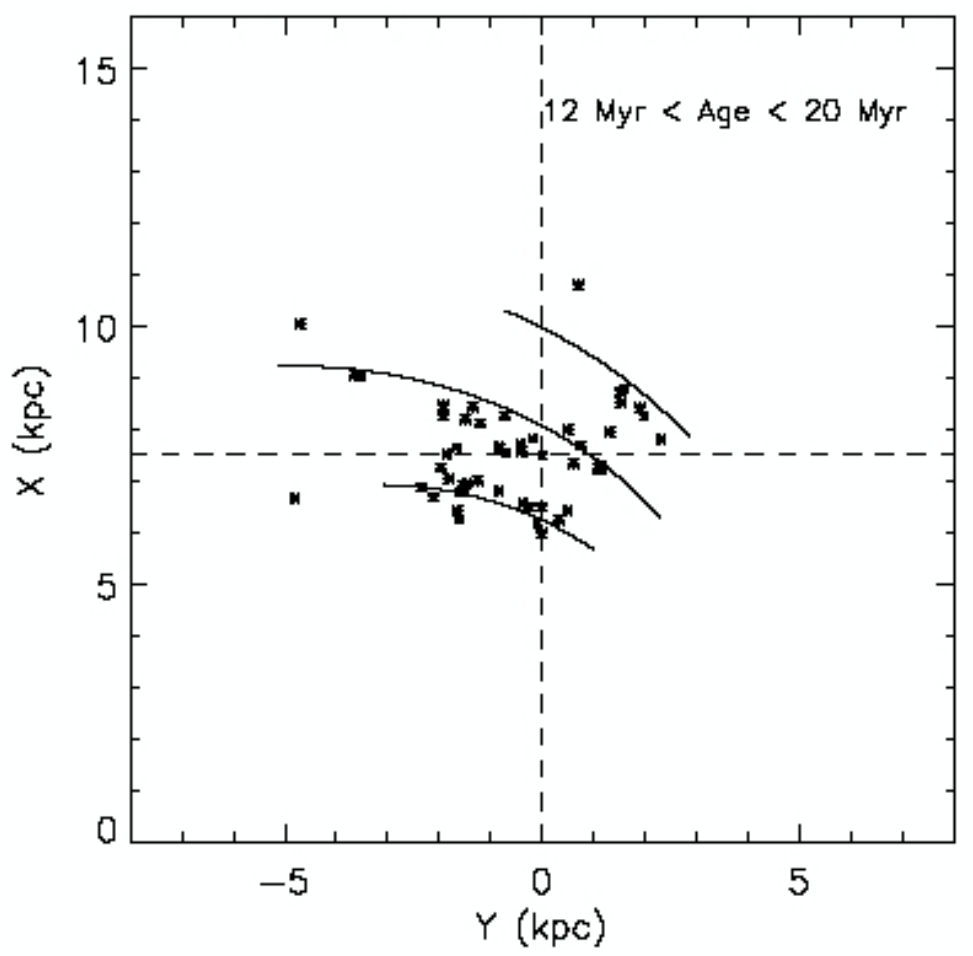

Figura 1.3: No diagrama são ilustrados os principais braços (Perseus, local e Sagitarius-Carina) obtidos a partir da localização de aglomerados abertos. $\mathrm{O}$ sol se situa no centro do diagrama $(\mathrm{x}=0, \mathrm{y}=0)$. São utilizados cerca de 109 aglomerdos abertos com idades inferiores a 10 milhões de anos. As coordenadas equatoriais dos aglomerados foram convertidas para coordenadas galácticas e depois para x,y galácticas considerando a distância.

\subsection{Aglomerados Abertos e Perspectivas Históricas}

Muito provavelmente os aglomerados abertos foram os primeiros a serem observados por estarem situados a uma pequena distância de nós, quando comparados aos aglomerados globulares. Alguns aglomerados como Plêiades (M45), Híades e Beehive (M44) são conhecidos desde a antiguidade. Homero (800 a. C.) mencionou Plêiades e Híades ${ }^{1}$ em sua obra "Ilíada". Plêiades que é um dos aglomerados abertos mais próximos da Terra, provavelmente o mais conhecido é tambem o mais visível a olho nu. Talvez por esse motivo tenha sido citado em textos na antiguidade. O conhecimento apurado sobre esse aglomerado e de importancia crucial em astronomia por possibilitar a determinaçao de distâncias a outros corpos celestes

A história da astronomia é permeada por mitos e lendas, o mito das Plêi-

\footnotetext{
${ }^{1}$ Ambas se localizam na constelação zodiacal de touro
} 
ades, também chamadas de "Sete Irmãs", que eram as filhas de Atlas, suicidaram-se pesarosas do triste destino de seu pai (Atlas), obrigado a carregar o mundo nas costas. Zeus compadecendo-se delas transformou-as em estrelas.

O mais fraco objeto já observado na antiguidade teria sido M41 (NGC 2287), por Aristóteles em 325 a.C, o que o transforma na primeira gravação de um aglomerado aberto. Ptolomeu encontrou o próximo objeto do qual se tem notícia em 138 d.C., o aglomerado M7 (NGC 6745) em Scorpião. Além de M7, ele descobriu outros 3 aglomerados, incluindo Coma Berenices, hoje catalogado como Melotte 111. O aglomerado de mais difícil identificação a olho nu foi possivelmente descoberto por Messier, o chamado Beehive, catalogado mais tarde como M44 (NGC 2632).

Foi somente no século XVII, com o uso do telescópio por Galileu Galilei (1564-1642), que cresce a compreensão acerca das nebulosas não desvendadas. Entendese que elas são constituídas por muitas estrelas de pouco brilho, invisíveis a olho nu. Galileu foi provavelmente o primeiro a observar tais objetos com um telescópio, acreditando que cada nebulosa no céu poderia ser resolvida individualmente como um conjunto de estrelas. Ao longo do século XVII foram feitas algumas descobertas esporádicas de novos aglomerados abertos.

\subsection{Catálogos de Aglomerados Abertos e o Catálogo DAML02}

Desde a primeira lista de nebulosas, compilada por Edmond Halley em 1715, o primeiro catálogo de aglomerados abertos foi definitivamente o catálogo de Messier, e até os dias de hoje encontram-se referências a respeito desse catálogo. A primeira versão do catálogo (1774) contava com cerca de 45 objetos. Outras versões foram publicadas posteriormente, mas a versão final (1781) continha aproximadamente 103 objetos, de M1 até M103. O catálogo de Messier também instigou outros astrôno- 
mos em suas próprias buscas, como por exemplo William Hershel. As informações do catálogo de Messier foram compiladas no catálogo de William Hershel, que acrescentou cerca de dois mil e trezentos novos objetos à lista. Hershel chegou a descobrir cerca de 2300 novos objetos.

Conforme as informações acerca de aglomerados abertos aumentaram, classificálos de forma adequada tornou-se uma necessidade e nesse contexto surgem os modernos catálogos do século XX. Um dos primeiros sistemas de classificação foi introduzido em 1930 por Harlow Shapley, membro da Universidade de Harvard. O catálogo aparecia simplesmente com o nome "Aglomerados de Estrelas". O catálogo continha cerca de duzentos e ciquenta aglomerados listando apenas informações fundamentais, ele foi usado durante muitas décadas e atualmente é considerado obsoleto.

O próximo catálogo importante a ser publicado veio apenas em 1958 por Alter [15], tendo edições posteriores em 1966, 1970 e 1981. Muitas das informações contidas nesse catálogo estão hoje disponíveis no $\mathrm{ADS}^{2}$, tratando não só de aglomerados abertos, como também de aglomerados globulares e associações.

Um dos mais recentes catálogos publicados é o "5th Lund-Strasbourg Star Catalogue" ou Lund Catalogue, que foi primeiramente publicado pelo observatório Lund por Gosta Lynga. O catálogo contém uma lista de 1151 aglomerados sendo identificados pela sigla IAU ${ }^{3}$ seguida pela designação tradicional da descoberta e do aglomerado. O catálogo Lynga foi útil por conter uma série de parâmetros importantes acerca dos aglomerados abertos, como idades e tamanho do aglomerado, além de excessos de cor.

Outro catálogo disponível que se pode citar é o $W E B D A^{4}$. O catálogo foi publicado por Jean-Claude Mermilliod [17] sendo que atualmente é mantido por Ernest Pauzen. O WEBDA permite o acesso fácil aos dados disponíveis sobre aglomerados abertos. O catálogo que aparece no livro Willman-Bell "Star Clusters" 2004, é um

\footnotetext{
${ }^{2}$ Sigla usada para Astronomical Data Service

${ }^{3}$ Em inglês International Astronomical Union

${ }^{4}$ Disponível em (http://obswww.unige.ch/webda/)
} 
outro exemplo de catálogo útil disponível.

Um dos catálogos mais modernos disponíveis atualmente, é o catálogo de W.S. Dias et. al. "New Catalog of Optically Visible Open Clusters and Candidates"6. O catálogo foi originalmente publicado pela revista "Astronomy and Astrophysics" 2002 [1]. Ele compila e atualiza os catálogos anteriores de Lynga [16] e de Mermilliod [17] (base de dados $W E B D A$ ). Dessa forma, se caracteriza por ser o mais atual catálogo de aglomerados abertos disponível, fornecendo informações sobre parâmetros cinemáticos (movimentos próprios médios e velocidades radiais médias), além de parâmetros fundamentais (distâncias, avermelhamentos e idades) de centenas de aglomerados abertos [[2], [3]] o que viabiliza estudos acerca da estrutura da Galáxia como por exemplo sobre a velocidade de rotação dos braços espirais, questão recentemente abordada por Dias e Lépine [14].

Na versão mais recente do catálogo (versão 2.7) são 1756 aglomerados listados. São apresentadas estimativas de diâmetros aparentes, parâmetros cinemáticos (movimento próprio médio e velocidade radial média) e fundamentais (distâncias, avermelhamentos e idades). O catálogo já apresenta uma atualização, principalmente no tocante a dados cinemáticos, porém distâncias e idades são determinadas para uma pequena porcentagem dos objetos. A seguir reproduzimos as estatísticas do catálogo DAML02:

- Aglomerados com diâmetros $1753(99 \%)$

- Aglomerados com distâncias $1003(57 \%)$

- Aglomerados com avermelhamentos $.984(56 \%)$

- Aglomerados com idades $.864(48 \%)$

\footnotetext{
${ }^{5}$ Daqui em diante nos referimos ao catálogo usando a denominação DAML02(Dias, Alessi, Moitinho e Lepine - 2002)

${ }^{6}$ Disponível em http://www.astro.iag.usp.br/ wilton
} 
- Aglomerados com distâncias, avermelhamentos e idades $850(48 \%)$

- Aglomerados com movimentos próprios $889(50 \%)$

- Aglomerados com velocidades radiais. $358(20 \%)$

- Aglomerados com velocidades radiais e movimentos próprios $347(19 \%)$

- Aglomerados com distâncias, idades, movimentos próprios e velocidades radiais. $314(17 \%)$

- Aglomerados com metalicidade. $143(8 \%)$

De acordo com os dados acima, ainda é necessário esforço no sentido de completar as informações acerca dos aglomerados listados, uma vez que distâncias e idades são determinadas para uma pequena quantidade dos objetos. Os objetivos do nosso trabalho referem-se exatamente a completeza do catálogo DAML02, no sentido de fazer com que ele possua um maior número de informações acerca dos aglomerados listados e nunca antes estudados, no tocante a parâmetros cinemáticos e fundamentais.

Apresentamos uma análise do ponto de vista cinemático para 857 aglomerados abertos listados na versão 2.3 do catálogo DAML02, sendo que para 319 casos determinamos movimentos próprios médios, desvio padrão $(\sigma)$ nas coordendas de movimentos próprios $(\mu \alpha \cos \delta$ e $\mu \delta)$ e probabilidades de pertinência individuais para cada estrela presente no campo do aglomerado. Os dados referentes às estrelas na área dos aglomerados foram obtidos do catálogo astrométrico $U C A C 2$ ) [9] que fornece informações precisas sobre movimentos próprios além da fotometria nas bandas $\mathrm{J}, \mathrm{H}$ e K (do infravermelho próximo obtidas do catálogo $2 M A S S^{7}[10]$ ). Para obtenção dos valores de movimentos próprios médios, desvios padrão $(\sigma)$ e probabilidades individuais de pertinência das estrelas na região dos aglomerados utilizamos o conhecido método de Zhao e He [8].

\footnotetext{
${ }^{7}$ Em inglês Two Micron All Sky Survey
} 
A partir das estrelas selecionadas como membros ${ }^{8}$ mostramos ser possível determinar distâncias e idades dos objetos em questão. Para isso julgamos ser importante a determinação do excesso de cor individual das estrelas nas bandas do infravermelho $J$ e $H$, o $E(J-H)$, que é obtido a partir do tipo espectral de estrelas membros como valor inicial para as iterações no diagrama cor-magnitude, através do conhecido método do main sequence fitting. Para obter esses parâmetros inicias desenvolvemos um programa, que efetua cálculos para distâncias e excessos de cor preliminares. Foram obtidas 32 soluções satisfatórias para os parâmetros fundamentais, sendo que são 11 os casos inéditos.

No Capítulo 2 apresentamos uma discussão introdutória de alguns métodos existentes para determinação de movimentos próprios e probabilidades de pertinência utilizados em nossos cálculos: o método de Vasilevskis [6], a implementação do método introduzida por Sanders em 1971 [7] e o já mencionado método de Zhao e He introduzido em 1990. Expomos de forma sucinta, alguns aspectos que levaram a formulação de tais métodos e sua aplicabilidade em nosso trabalho. No próximo Capítulo, tratamos do método empregado por nós para a determnação dos parâmetros fundamentais (distâncias, excessos de cor e idades) abordando o método do main sequence fitting. Discutimos a aplicação e uso de tal método em nosso trabalho bem como suas limitações. Os Capítulos 4 e 5 dedicam-se respectivamente à apresentação dos resultados obtidos para movimentos próprios médios dos aglomerados, valores de sigma e probabilidades de pertinência das estrelas na região dos aglomerados estudados além dos parâmetros fundamentais. Fazemos em ambos capítulos uma comparação entre os nossos resultados e aqueles que se encontram disponíveis na literatura para os movimentos próprios, distâncias excessos de cor e idades. Por fim, procedemos com uma breve discussão para alguns dos casos sem solução.

\footnotetext{
${ }^{8}$ Selecionamos como membros as estrelas que apresentam probabilidade de pertinência superior ou igual a $51 \%$
} 


\section{Capítulo 2}

\section{Movimentos Próprios e probabilidades}

\section{de pertinência}

\subsection{Determinação de Movimentos Próprios e proba- bilidades de pertinência: A evolução do Método}

Ao observarmos uma determinada região do céu que contém um aglomerado aberto, percebemos que ali não existem apenas estrelas que o constituem, mas também estrelas intrusas, que não fazem parte do aglomerado e não mantém com seus membros nenhum tipo de vínculo. Dessa maneira, no estudo de aglomerados abertos é de suma importância separar as estrelas que os constituem de fato, daquelas que estão apenas em seu campo de visada (estrelas de fundo ou de frente, projetadas na região observada do aglomerado).

Embora pareça ser algo simples, a separação entre estrelas membros e intrusas no campo de um aglomerado aberto tem sido tratada como um problema clássico em astronomia. A presença dessas estrelas intrusas, prejudica seriamente a interpretação dos dados referentes a estes objetos, especialmente no que se refere à determinação 
de idades a partir da utilização do ponto de turn-off ${ }^{1}$ na sequência principal [18].

Durante os primeiros estudos acerca dos aglomerados abertos muito pouco se usou de métodos estatísticos na análise do movimento próprio ou do diagrama de movimentos próprios, conhecido como $\mathrm{VPD}^{2}$. De fato, o uso de critérios duvidosos, como por exemplo a posição da estrela no céu ou no diagrama $\mathrm{H}-\mathrm{R}^{3}$, colocavam em risco os dados sobre os membros do aglomerado. Os critérios utilizados anteriormente não faziam menção à óbvia presença das estrelas de campo.

Atualmente, vários são os critérios empregados a fim de se obter uma boa separação entre estrelas membro e intrusas no campo de um aglomerado aberto. A maior parte das análises se baseia em dados fotométricos ou cinemáticos (velocidades radiais e movimentos próprios respectivamente), sendo que a utilização deste último critério não inviabiliza a procura por estrelas peculiares no aglomerado [19].

O primeiro método desenvolvido com a finalidade de separar as duas populações de estrelas presentes no campo de visada de um aglomerado aberto usando movimentos próprios é da autoria de Vasilevskis e Rach em 1957 [6]. Foi utilizado um modelo simples no qual se assume uma distribuição homogênea dos movimentos próprios tanto do aglomerado como das estrelas intrusas no campo de observação. O método resultou em uma boa estimativa do número de estrelas pertencentes ao aglomerado e em um bom valor para o desvio padrão dos movimentos próprios do aglomerado. Posteriormente, Vasilevskis et. al. [20] introduziram um refinamento ao método considerando probabilidades individuais de pertinência das estrelas ao aglomerado. O modelo se baseia na superposição de uma função de freqüência binormal circular (movimentos próprios das estrelas do aglomerado) e uma função de distribuição de frequência binormal elíptica (movimentos próprios das estrelas intrusas). A probabilidade de pertinência era dada comparando-se o movimento próprio de cada estrela ao movimento próprio médio do

\footnotetext{
${ }^{1}$ Do inglês ponto de virada

${ }^{2}$ Do inglês Vector Proper Motion Diagram

${ }^{3}$ Diagrama Hertzprung-Hussel
} 
aglomerado.

Aproximadamente 13 anos depois, Sanders [7] publicou um estudo que propunha aliar ao método de Vasilevskis as facilidades da ferramenta computacional. A adaptação do método de Vasilevskis para um microcomputador permitia maior rigor estatístico ao ser aplicado o Princípio da Verossimilhança à distribuição e portanto fornecia valores consistentes para as probabilidades de pertinência das estrelas ao aglomerado. O método de Sanders foi posteriormente examinado por Slovak em 1977 [21], e por Dias em 2000 [22], principalmente no que diz respeito à estabilidade da solução. Em 1990 Zhao e He [8] introduziram uma informação adicional ao modelo ao considerar diferentes precisões para os movimentos próprios individuais das estrelas utilizadas. Ao ser aplicado o método, pode-se obter como resultados a probabilidade de cada estrela pertencer ao aglomerado, o movimento próprio médio do aglomerado e o movimento próprio médio da amostra de estrelas intrusas.

\subsection{O Método para o Cálculo de Movimentos Próprios e probabilidades de pertinência}

A partir da determinação do movimento próprio médio do aglomerado, é possível inferir a probabilidade de pertinência dos membros que compõe a amostra de estrelas, e assim separar as duas populações: estrelas membro e estrelas intrusas. Todas as análises feitas nesse trabalho foram baseadas no software desenvolvido por Dias, essencialmente adotamos o método de Sanders [7] mas para utilizar as precisões dos movimentos próprios individuais das estrelas o software utiliza o método estatístico de Zhao e He [8].

Dada uma amostra de $\mathrm{N}$ movimentos próprios $\mu_{x 1}, \mu_{y 1} ; \ldots ; \mu_{x n}, \mu_{y n}$, a função de Máxima Verossimilhança $L$ é dada pela Eq. 2.1, sendo que $\mu_{x 1}$ e $\mu_{x n}$ representam o movimento próprio na coordenada $x$ da primeira e da e-nésima estrela ; 
$\mu_{y 1}$ e $\mu_{y n}$ representam o movimento próprio na coordenada $y$ da primeira e da e-nésima estrela.

$$
L=\prod_{j=1}^{N} \phi\left(\mu_{x j}, \mu_{y j}\right) .
$$

Temos que o Princípio da Máxima Verossimilhança se aplica à mesma função de distribuição de movimentos próprios baseada no modelo de Vasilevskis et. al. [20] em que se somam a distribuição de movimentos próprios das estrelas intrusas (função normal elíptica) e membros do aglomerado (função normal circular):

$$
\begin{aligned}
\phi\left(\mu_{x j}, \mu_{y j}\right)= & \frac{n_{c}}{2 \pi \sigma^{2}} \exp \left\{-\frac{1}{2}\left[\left(\frac{\mu_{x j}-\bar{\mu}_{x c}}{\sigma}\right)^{2}+\left(\frac{\mu_{y j}-\bar{\mu}_{y c}}{\sigma}\right)^{2}\right]\right\}+ \\
& \frac{n_{i}}{2 \pi \sigma_{x} \sigma_{y}} \exp \left\{-\frac{1}{2}\left[\left(\frac{\mu_{x j}-\bar{\mu}_{x i}}{\sigma_{x}}\right)^{2}+\left(\frac{\mu_{y j}-\bar{\mu}_{y i}}{\sigma_{y}}\right)^{2}\right]\right\},
\end{aligned}
$$

onde:

$n_{i}$ e $n_{c}$ correspondem ao número de estrelas do aglomerado e intrusas respectivamente; $\sigma$ é o desvio padrão dos movimentos próprios das estrelas do aglomerado;

$\sigma_{x}$ e $\sigma_{y}$, o desvio padrão em x e y dos movimentos próprios das estrelas intrusas;

$\mu_{x j}$ e $\mu_{y j}$ representam o movimento próprio em x e y das estrelas da amostra;

$\bar{\mu}_{x c}$ e $\bar{\mu}_{y c}$ são o movimento próprio médio em x e y das estrelas dos aglomerado;

$\bar{\mu}_{x i}$ e $\bar{\mu}_{y i}$ representam o movimento próprio médio em x e y das estrelas intrusas.

Computacionalmente é conveniente normalizar a população de forma que:

$$
n_{c}+n_{i}=1
$$

De acordo com o modelo de Vasilevskis et. al. [20], $\sigma, \sigma_{x}$ e $\sigma_{y}$ são a variância dos movimentos próprios, que consistem das variâncias intrínsecas e erros 
observacionais. Desde que erros observacionais nas direções x e y são praticamente os mesmos [31], temos:

$$
\begin{gathered}
\sigma^{2}=\sigma_{o}^{2}+\epsilon^{2} \\
\sigma_{x}^{2}=\sigma_{x o}^{2}+\epsilon^{2} \\
\sigma_{y}^{2}=\sigma_{y o}^{2}+\epsilon^{2},
\end{gathered}
$$

onde o subscrito "o" denota a variância intrínseca e " $\epsilon$ " os desvios observados.

Se " $\epsilon$ " for diferente de estrela para estrela, $\sigma, \sigma_{x}$ e $\sigma_{y}$ não são constantes para todos os membros do aglomerado e as estrelas do campo respectivamente. Dessa maneira não é teoricamente rigoroso utilizar a Eq. $2.2 \mathrm{com} \sigma, \sigma_{x}$ e $\sigma_{y}$ desconhecidos para a determinação de movimentos próprios. De acordo com Zhao e He podemos resolver este problema ao inserir as Eq. 2.3 , 2.4 e 2.5 na Eq. 2.2 obtendo:

$$
\begin{gathered}
\phi\left(\mu_{x_{j}}, \mu_{y_{j}}\right)=\frac{n_{c}}{2 \pi\left(\sigma_{o}^{2}+\epsilon_{j}^{2}\right)} \exp \left\{-\frac{1}{2}\left[\frac{\left(\mu_{x_{j}}-\bar{\mu}_{x_{c}}\right)^{2}}{\sigma_{o}^{2}+\epsilon_{j}^{2}}+\frac{\left(\mu_{y_{j}}-\bar{\mu}_{y_{c}}\right)^{2}}{\sigma_{o}^{2}+\epsilon_{j}^{2}}\right]\right\}+ \\
\frac{1-n_{c}}{2 \pi\left(\sigma_{x o}^{2}+\epsilon_{j}^{2}\right)^{1 / 2}\left(\sigma_{y o}^{2}+\epsilon_{j}^{2}\right)^{1 / 2}} \exp \left\{-\frac{1}{2}\left[\frac{\left(\mu_{x_{j}}-\bar{\mu}_{x_{i}}\right)^{2}}{\sigma_{x o}^{2}+\epsilon_{j}^{2}}+\frac{\left(\mu_{y_{j}}-\bar{\mu}_{y_{i}}\right)^{2}}{\sigma_{y o}^{2}+\epsilon_{j}^{2}}\right]\right\} .
\end{gathered}
$$

Existem 8 parâmetros a serem determinados nesta equação: $n_{c}, \mu_{x i}, \mu_{y i}$, $\mu_{x c}, \mu_{y c}, \sigma_{x i}, \sigma_{y i}$ e $\sigma_{c}$. A diferença entre a equação 2.2 e 2.6 é que a variância intrínseca $\sigma_{o}^{2}$ e $\left(\sigma_{x o}^{2}, \sigma_{y o}^{2}\right)$ são constantes para o campo, sendo independentes para qualquer membro do aglomerado ou do campo. De acordo com o Princípio da Máxima Verossimilhança na Eq. 2.1 temos que:

$$
\frac{\partial \ln L}{\partial q_{j}}=\frac{\partial}{\partial q_{j}}\left(\sum \ln \phi_{j}\right)=0
$$

onde $q_{j}$ pode ser qualquer um dos oito parâmetros da Eq. 2.6 a serem derivados.

Aplicando a equação 2.7 à equação 2.6 a cada parâmetro $j$ temos o sistema 
de 8 equações não lineares a ser resolvido:

$$
\begin{aligned}
n_{c}: \sum \phi^{-1}\left[\alpha\left(\sigma_{x o}^{2}+\epsilon^{2}\right)^{-1 / 2}\left(\sigma_{y o}^{2}+\epsilon^{2}\right)^{-1 / 2}-\beta\left(\sigma_{o}^{2}+\epsilon^{2}\right)^{-1}\right] & =0 \\
\mu_{x i}: \sum \phi^{-1} \alpha\left(\mu_{x}-\mu_{x i}\right)\left(\sigma_{x o}^{2}+\epsilon^{2}\right)^{-3 / 2}\left(\sigma_{y o}^{2}+\epsilon^{2}\right)^{-1 / 2} & =0 \\
\mu_{y i}: \sum \phi^{-1} \alpha\left(\mu_{y}-\mu_{y i}\right)\left(\sigma_{y o}^{2}+\epsilon^{2}\right)^{-1 / 2}\left(\sigma_{y o}^{2}+\epsilon^{2}\right)^{-3 / 2} & =0 \\
\mu_{x c}: \sum \phi^{-1} \beta\left(\mu_{x}-\mu_{x c}\right)\left(\sigma_{o}^{2}+\epsilon^{2}\right)^{-2} & =0 \\
\mu_{y c}: \sum \phi^{-1} \beta\left(\mu_{y}-\mu_{y c}\right)\left(\sigma_{o}^{2}+\epsilon^{2}\right)^{-2} & =0 \\
\sigma_{x i}: \sum \phi^{-1} \alpha\left(\sigma_{x o}^{2}+\epsilon^{2}\right)^{-3 / 2}\left(\sigma_{y o}^{2}+\epsilon^{2}\right)^{-1 / 2}\left[\left(\mu_{x i}-\mu_{x}\right)^{2}\left(\sigma_{x o}^{2}+\epsilon^{2}\right)^{-1}-1\right] & =0 \\
\sigma_{y i}: \sum \phi^{-1} \alpha\left(\sigma_{x o}^{2}+\epsilon^{2}\right)^{-1 / 2}\left(\sigma_{y o}^{2}+\epsilon^{2}\right)^{-3 / 2}\left[\left(\mu_{y}-\mu_{y i}\right)^{2}\left(\sigma_{x o}^{2}+\epsilon^{2}\right)^{-1}-1\right] & =0 \\
\sigma_{c}: \sum \phi^{-1} \beta\left(\sigma_{o}^{2}+\epsilon^{2}\right)^{-2}\left\{\left[\left(\mu_{y}-\mu_{y c}\right)^{2}+\left(\mu_{x}-\mu_{x c}\right)^{2}\right]\left(\sigma_{o}^{2}+\epsilon^{2}\right)^{-1}-2\right\} & =0,
\end{aligned}
$$

onde:

$$
\begin{aligned}
& \beta_{j}=\exp \left\{-\frac{1}{2}\left[\frac{\left(\mu_{x_{j}}-\bar{\mu}_{x_{c}}\right)^{2}}{\sigma_{o}^{2}+\epsilon_{j}^{2}}+\frac{\left(\mu_{y_{j}}-\bar{\mu}_{y_{c}}\right)^{2}}{\sigma_{o}^{2}+\epsilon_{j}^{2}}\right]\right\} e \\
& \alpha_{j}=\exp \left\{-\frac{1}{2}\left[\frac{\left(\mu_{x_{j}}-\bar{\mu}_{x_{i}}\right)^{2}}{\sigma_{x o}^{2}+\epsilon_{j}^{2}}+\frac{\left(\mu_{y_{j}}-\bar{\mu}_{y_{i}}\right)^{2}}{\sigma_{y_{o}}^{2}+\epsilon_{j}^{2}}\right]\right\} .
\end{aligned}
$$

Para facilitar os sub-índices " $\hat{\jmath}$ " que se encontrariam nas quantidades $\phi_{j}, \alpha_{j}, \beta_{j}, \epsilon_{j}$ e $\mu_{x j}, \mu_{y j}$, devido a cada estrela, foram suprimidos; $\sum$ é a somatória sobre todas as estrelas, ou seja de $j=1$ até $N$.

Após terem sido iniciadas as iterações, o método nos exige que os eixos x e y sejam paralelos aos eixos principais da distribuição elíptica dos movimentos próprios. A rotação de eixos é necessária graças a existência de um termo cruzado que dificulta a aplicação do princípio da Máxima Verossimilhança á função de distribuição dos movimentos próprios. Assim antes de se aplicar as equações devemos proceder com uma 
rotação de eixos. Um método de se fazer tais rotações é proposto por Vasilevskis et. al. [23] e é aplicado nos códigos do software de Dias.

O processo de resolução do sistema de equações não lineares é o mesmo descrito por Sanders [7], ou seja, um processo iterativo. Valores iniciais aproximados são fornecidos para todos os parâmetros de acordo com o Método de Vasilevskis [6], e as equações iteradas passo a passo. Variam-se os parâmetros associados até que o valor que melhor satisfaça a equação seja obtido, esse então substitui seu valor inicial e o processo se repete para a próxima equação, onde um valor melhor para o parâmetro associado é encontrado e assim por diante. Quando o processo progrediu de forma que as oito equações tenham sido iteradas sem nenhuma mudança nos valores dos parâmetros, esses são por sua vez adotados como os valores que melhor se ajustam à distribuição, de acordo com o modelo [7]. O método de iteração utilizado é notavelmente estável como podemos verificar em [22].

A probabilidade de pertinência de cada estrela é definida de acordo com [8]:

$$
P_{j}=\frac{n_{c} \beta_{j}}{2 \pi\left(\sigma_{o}^{2}+\epsilon^{2}\right) \phi_{j}}
$$

Ao final do processo o programa fornece o movimento próprio médio do aglomerado, probabilidades de pertinência para cada estrela presente na amostra, o movimento próprio médio da amostra de estrelas intrusas e o valor do desvio padrão para o movimento próprio das estrelas do aglomerado e intrusas. 


\subsection{Dados utilizados e análise dos resultados forneci- dos pelo programa}

Como o programa calcula uma função de distribuição para os movimentos próprios das estrelas (do aglomerado mais intrusas), é necessário obtermos dados dos movimentos próprios de todas as estrelas na região do aglomerado analisado. Em nosso trabalho, as informações necessárias sobre as estrelas na área dos aglomerados foram extraídas do Catálogo UCAC2 [9].

O UCAC2 foi selecionado porque no total, são fornecidas posições e movimentos próprios (bem como informações nas bandas J,H e K do catálogo 2 MASS) para mais de 40 milhões de estrelas com magnitudes (banda R) até 16 . O catálogo cobre todo o céu até declinações $+40^{\circ}$, chegando a declinações $+52^{\circ}$ em certas regiões. Seus movimentos próprios encontram-se no sistema Hipparcos com erros nominais em torno de 1 a 3 mas $/ \mathrm{yr}^{4}$ para estrelas com magnitude 12, e por volta de 4 a 7 mas/yr para estrelas com magnitude entre 12 e 16. A boa precisão dos movimentos próprios das estrelas e o desvio sistemático esperado abaixo de 1 mas/yr [9], ou seja, praticamente desprezível bem como o fato de ser o mais completo e atual catálogo de movimentos próprios, torna o catálogo $U C A C 2$ uma fonte de informações fundamental para nossos estudos.

Em todos os casos utilizamos para a extração das estrelas numa determinada região as facilidades da ferramenta $V i z i e R^{5}$. É importante ressaltar que o raio adotado para extração das estrelas não seguiu uma regra uma vez que poderia haver necessidade de aumentar ou diminuir a contaminação de estrelas intrusas.

A análise dos resultados fornecidos pelo programa é feita a partir do desvio padrão $\sigma$ para os valores de $\mu \alpha \cos (\delta)$ e $\mu \delta$ que são dados de saída do programa. O erro

\footnotetext{
${ }^{4}$ mas/yr é o símbolo comumente usado como unidade de movimentos próprios e significa milisegundo de arco por ano.

${ }^{5}$ Disponível em http://vizier.u-strasbg.fr/viz-bin/VizieR-3
} 
associado ao valor de $\sigma$ é dado por

$$
\text { erro }=\frac{\sigma}{\sqrt{n_{c}-1}}
$$

O software também apresenta duas ferramentas que podem ser utilizadas interativamente de modo que os resultados obtidos para as pertinências das estrelas são apresentados no diagramas dos vetores movimentos próprios ( $V P D)$ e também num diagrama cor-magnitude $(C M D)^{6}$ a partir dos dados fotométricos do $2 M A S S$, além da distribuição das probabilidades de pertinência de cada estrela. É importante lembrar que damos maior peso à análise cinemática e por isso o VPD é fundamental. Em contrapartida, é possível muitas vezes utilizar o $C M D$ como ferramenta auxiliar na solução astrométrica. As figuras 2.1 e 2.2 dão uma clara idéia das ferramentas usadas na avaliação dos resultados de saída.
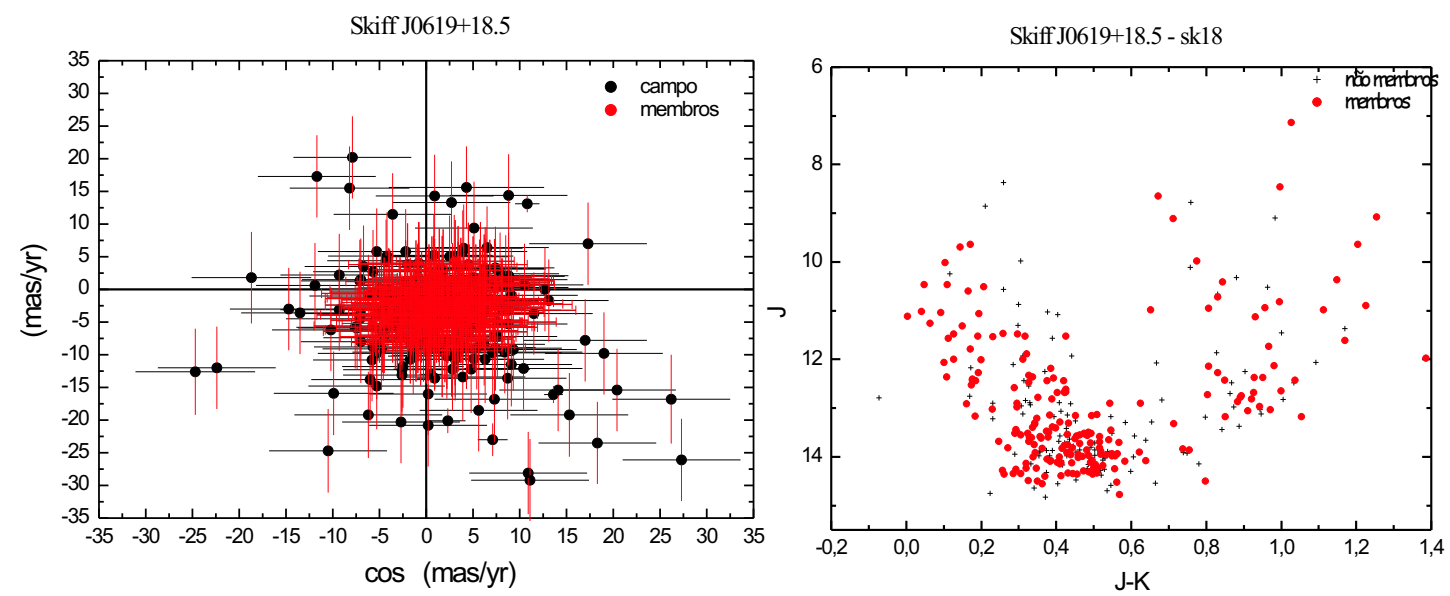

Figura 2.1: Diagramas VPD (à esquerda) e CMD (à direita) para o aglomerado Skiff J0619+18.5, ferramentas de análise do programa utilizado para o cálculo de movimentos próprios médios dos aglomerados. Em vermelho as estrelas com probabilidades de pertinência maiores que 51\%. O diagrama VPD mostra pontos referentes aos valores de movimentos próprios das estrelas nas duas coordenadas $\mu \alpha \cos \delta$ e $\mu \delta$ em mas/yr, enaquanto que o diagrama CMD mostra os pontos referentes a magnitude das estrelas nas bandas J-H X J do infravermelho próximo.

De acordo com o gráfico de probabilidades da figura 2.2 para o aglomerado Skiff J0619+18.5, podemos perceber a separação entre duas populações de estrelas: uma

\footnotetext{
${ }^{6}$ Do inglês Colour Magnitude Diagram
} 


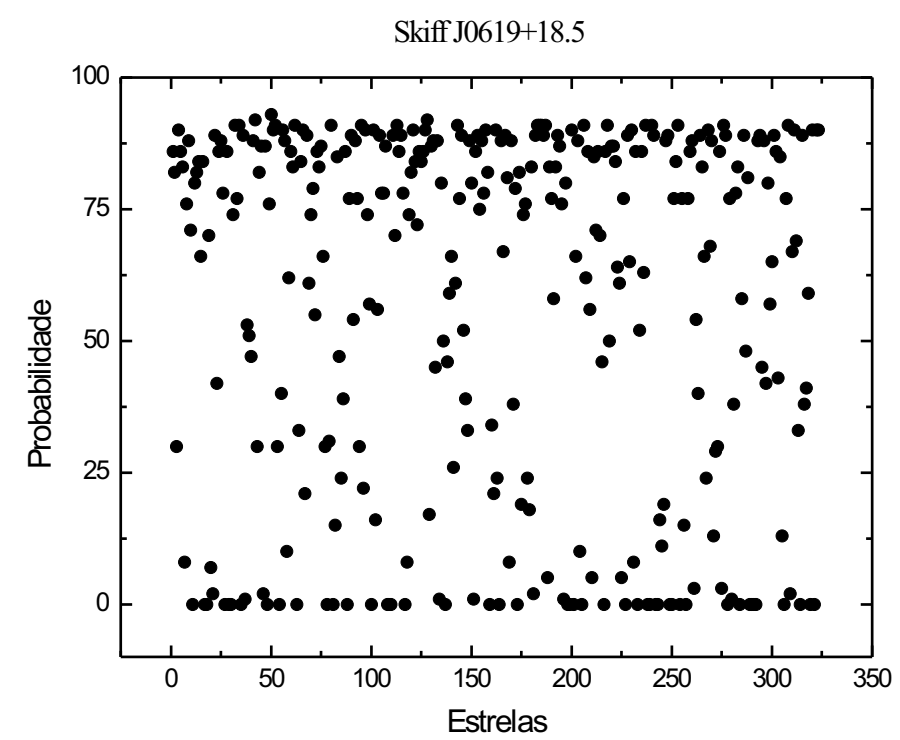

Figura 2.2: Diagrama de probabilidades de pertinência das estrelas ao aglomerado Skiff J0619+18.5. Uma das ferramentas mais importantes na análise dos dados de saída do programa, o diagrama mostra as probabilidades de pertinência das estrelas do campo ao aglomerado.

com probabilidades menores que $51 \%$ e próximas de zero, e outra com probabilidades superiores a $51 \%$ e mais próximas de $100 \%$.

\subsection{Confiabilidade do Método}

Os testes de confiabilidade do método podem ser melhor conferidos em um artigo recentemente publicado pelo nosso grupo [4] ${ }^{7}$. Nesse artigo, são apresentados os resultados de determinação de movimentos próprios médios e análise de probabilidades de pertinência para uma amostra de centenas de aglomerados com distâncias conhecidas em nosso catálogo. É importante ressaltar que a amostra estudada nessa seção trata de aglomerados diferentes da amostra estudada nesse projeto.

Todos os aglomerados com distâncias conhecidas e com $\delta \leq 52^{0}$ foram investigados, e os movimentos próprios médios absolutos foram obtidos para 428 aglo-

\footnotetext{
${ }^{7}$ Disponível em http://www.astro.iag.usp.br/ wilton
} 
merados, usando os dados provenientes do Catálogo UCAC2 e o método estatístico de Zhao \& He [8]. Para 75 aglomerados abertos, trata-se do primeiro resultado obtido, significando um incremento de $13 \%$ na amostra de aglomerados abertos com movimentos próprios médios determinados.

Os resultados em geral concordam com outras determinações de movimentos próprios médios. Em particular, nossos resultados concordam melhor com os resultados previamente obtidos em [[2] e [3]] baseados no Catálogo Tycho2 [24].

A partir da figura 2.3 a seguir podemos visualizar mellhor a comparação dos movimentos próprios médios obtidos pelo grupo com outros estudos. Para checar a qualidade dos nossos movimentos próprios obtidos, comparamos diferenças em componentes de ascenção reta e declinação para cada aglomerado, de acordo com os erros formais calculados de acordo com a relação:

$$
\begin{aligned}
& D=\frac{\left(\mu \alpha \cos \delta_{U C A C 2}-\mu \alpha \cos \delta_{\text {literatura }}\right)^{2}}{\left(\sigma_{\mu \alpha \cos \delta_{U C A C 2}}\right)^{2}+\left(\sigma_{\mu \alpha \cos \delta_{\text {literatura }}}\right)^{2}} \\
& +\frac{\left(\mu \delta_{U C A C 2}-\mu \delta_{\text {literatura }}\right)^{2}}{\left(\sigma_{\mu \delta_{U C A C 2}}\right)^{2}+\left(\sigma_{\mu \delta_{\text {literatura }}}\right)^{2}} .
\end{aligned}
$$

Em todos os casos, quase todos os valores de $(D)$ ficam entre 2.5 e 3.0. No entanto, podemos dizer seguramente que não há distinção entre os movimentos próprios comparados dentro dos erros estimados.

Essencialmente, os mesmos desvios padrão em movimentos próprios que são encontrados na literatura são reproduzidos aqui para esses aglomerados abertos, porém, com um número muito maior de estrelas membro identificadas. O número total de estrelas estudadas é de cerca de cento e cinquenta mil das quais quarenta e duas mil foram consideradas membros astrométricos. Podemos notar um avanço no uso do catálogo UCAC2 em detrimento do Catálogo Tycho2, no que se refere à quantidade de estrelas englobadas na análise, portanto um número de membros astrométricos muito 
maior, chegando a 42 mil estrelas com magnitudes até 16, contra apenas 7 mil estrelas com magnitudes até 12 no Tycho2. Os dois possuem a mesma dispersão típica de 3mas/yr em movimentos próprios.

Também podemos vizualizar uma comparação entre os resultados obtidos, no diagrama da figura 2.4 que mostra simultaneamente as diferenças em $\mu \alpha \cos \delta$ e $\mu \delta$. No caso dos aglomerados Harvard 10, IC 4665, Ruprecht 147 e Trumpler 10, parece que nossa análise foi incapaz de distinguir entre membros e estrelas de fundo, provavelmente pelo tamanho dos aglomerados ou por estarem muito próximos em distância, o que compromete a aplicação do método descrito na seção 2.2. Boas soluções para esses aglomerados são fornecidas por DLA ${ }^{8}$ usando dados do catálogo Tycho2.

O emprego do método descrito e o uso do Catálogo UCAC2 para determinação dos movimentos próprios médios e probabilidades de pertinência fica justificado graças ao que foi exposto nesta seção. O método foi aplicado a todos os casos em que não se encontram distâncias e idades no catálogo DAML02 e os resultados são apresentados no Capítulo 4.

\footnotetext{
${ }^{8}$ Designação usada para abreviar Dias et. al. (2001,2002).
} 

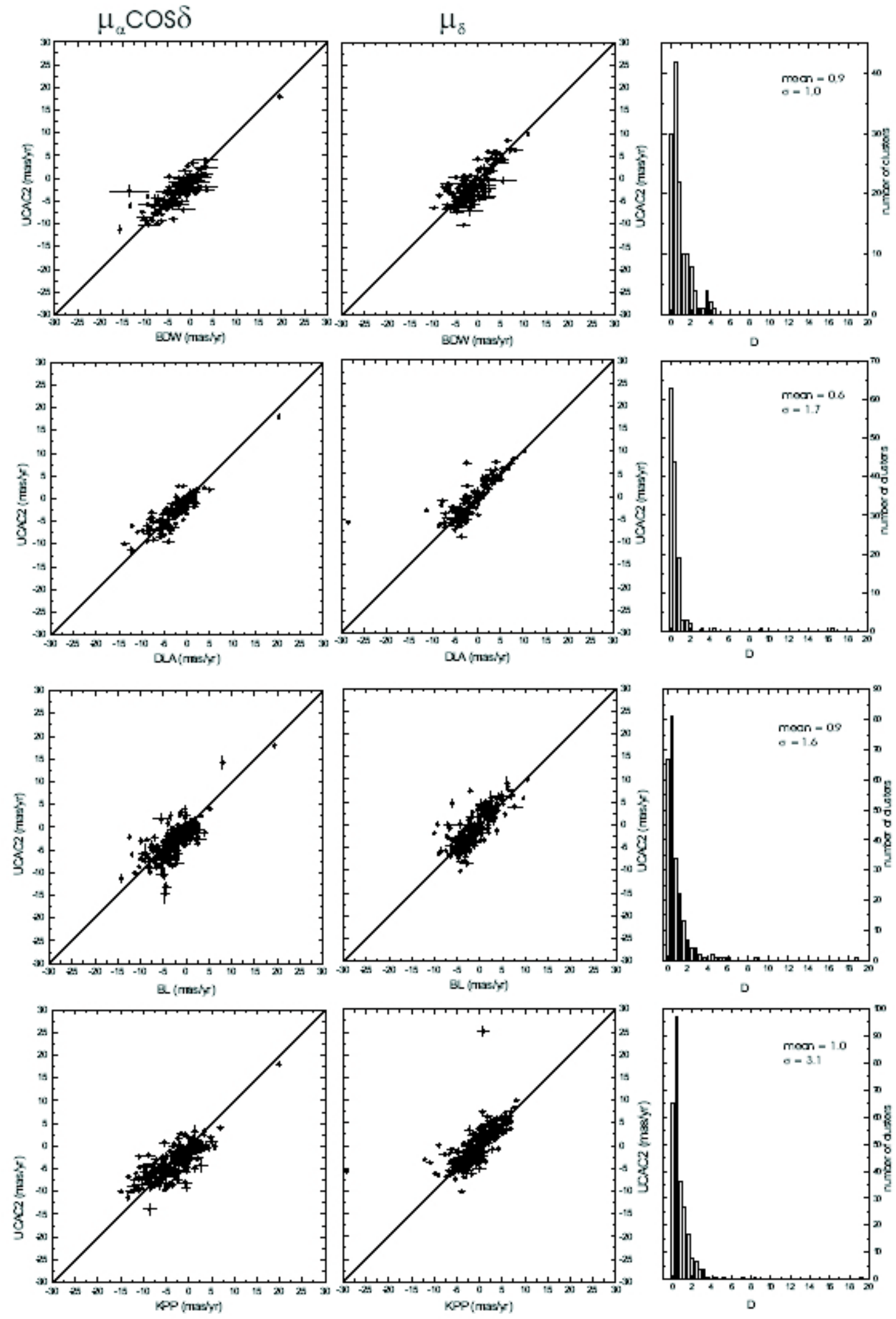

Figura 2.3: Comparação dos nossos resultados para movimentos próprios médios com aqueles fornecidos por Baumgardt et. al. (2000); Dias et. al. (2001,2002); Beshenov e Loktin(2004), Kharchenko et. al. (2003)(BDW, DLA, BL, KPP, respectivamente). As linhas de $45^{\circ}$ representam movimentos próprios iguais. À direita temos a distribuição das diferenças em movimentos próprios para cada comparação, levando em consideração componentes de ascenção reta e declinação pesados pelos erros formais (equação 2.10); eles indicam que não há distinção entre os movimentos próprios comparados dentro dos erros estimados. 


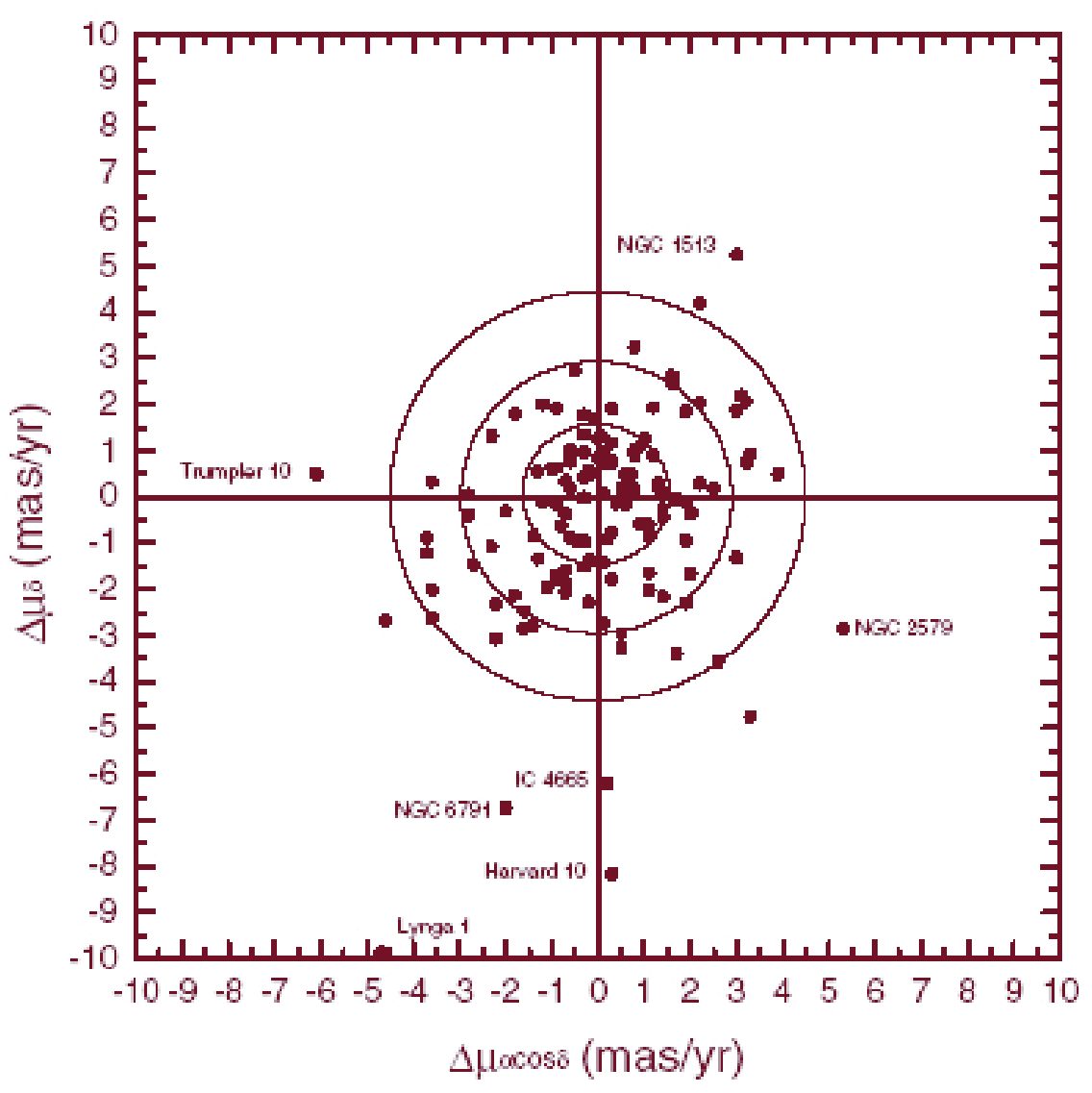

Figura 2.4: Diferenças em movimento próprio médio absoluto (UCAC2-Dias et. al. 2001, 2002)[2] em $\mu \alpha \cos \delta$ e $\mu \delta$. Os círculos mostram as regiões de desvio padrão correspondentes a um, dois e três $\sigma$ obtidas neste estudo. 


\section{Capítulo 3}

\section{Parâmetros Fundamentais}

\subsection{Distâncias e Idades}

Para a determinação de distâncias e idades é importante ter aglomerados com membros muito bem resolvidos para que possa ser adequadamente estudado e empregado como instrumento de valor em estudos dos mais variados ramos da astronomia. A determinação de distâncias e idades constitui a segunda etapa do projeto, uma vez que depende da atribuição das probabilidades de pertinência a cada estrela estudada em um aglomerado, a partir dos dados cinemáticos.

Após selecionarmos os membros de cada aglomerado, seleção essa feita com base no valor atribuído à probabilidade de pertinência individual de cada estrela ao aglomerado (consideramos pertinentes ao aglomerado somente estrelas com valores de probabilidade acima de 51\%), construimos para análise fotométrica, os diagramas cor-magnitude de cada aglomerado nas bandas $J, H$ e $K$ do infravermelho. Através de uma busca detalhada na base de dados $S I M B A D^{1}$ é possível extrair os tipos espectrais (denotaremos $T E{ }^{\prime}{ }^{2}$ ) de algumas estrelas de cada aglomerado, e com o uso desses TE's estimar as cores intrínsecas por interpolação linear com algumas tabelas (ver detalhes

\footnotetext{
${ }^{1}$ Disponível em http://simbad.u-strasbg.fr/sim-fid.pl

${ }^{2}$ De: tipo espectral
} 
em Bessell and Brett [25] e (J. Koornneef 1983) [26]. ) relacionando TE's e cores nas bandas $\mathrm{J}$ e $\mathrm{H}$ do infravermelho. Dos valores fornecidos para cada estrela nas bandas $\mathrm{J}$ e H do catálogo 2MASS [10], obtemos as cores observadas de cada estrela. Uma vez com as cores intrínsecas, obtemos o excesso de cor individual nessas bandas através da expressão:

$$
E(J-H)=(J-H)_{o b s}-(J-H)_{i n t},
$$

onde $(J-H)_{o b s}$ é a cor observada, $(J-H)_{i n t}$ é a cor intrínseca e $E(J-H)$ é o excesso de cor nas bandas do infravermelho $J$ e $H$. No visível temos uma expressão equivalente:

$$
E(B-V)=(B-V)_{o b s}-(B-V)_{i n t},
$$

sendo $(B-V)_{\text {obs }}$ a cor observada,$(B-V)_{\text {int }}$ a cor inrtínseca e $E(B-V)$ o excesso de cor no visível.

A distribuição dos excessos de cor pode então ser usada para deduzir o avermelhamento médio para cada aglomerado. Lembramos que essa média é feita apenas com algumas estrelas do aglomerado, uma vez que não se tem o $T E$ de todas as estrelas de cada aglomerado. O avermelhamento pode ser calculado através da bem conhecida expressão que relaciona excesso de cor $(E(B-V))$ com avermelhamento $\left(A_{v}\right)$ : de Mihalas [27].

$$
A_{v}=3.1 * E(B-V),
$$

e usando as relações derivadas das taxas de absorção [28]:

$$
\frac{E(J-H)}{E(B-V)}=0.309
$$




$$
\frac{E\left(J-K_{s}\right)}{E(B-V)}=0.488
$$

onde $E\left(J-K_{s}\right)$ é o excesso de cor nas bandas do infravermelho $J$ e $K$.

Dessa maneira obtemos uma relação direta para o avermelhamento usando os valores no infravermelho a partir das equações 3.4 e 3.3 :

$$
<A_{v}>=10 * E(J-H) .
$$

De uma maneira similar usamos os TE's para determinar as magnitudes absolutas, e com as magnitudes absolutas e aparentes determinar a respectiva distribuição do módulo de distância, das estrelas membros de um aglomerado:

$$
m-M=5 \log d-5+A_{v},
$$

onde $m$ e $M$ são as magnitudes aparente e absoluta respectivamente, $d$ é a distância e $A_{v}$ a extinção interestelar.

Uma vez traçado o $C M D$ para um dado aglomerado, obtemos a distância através do método do main sequence fitting. A partir dos diagramas ( $\mathrm{J}-\mathrm{H}$ x J) e (J-K x J) ajustando uma isócrona de idade zero $(Z A M S)$, é possível determinar o valor para a distância. É necessário ajustar os valores tanto de distância quanto de excesso de cor $E(J-H)$, de modo que a isócrona se ajuste às estrelas na seqüência principal do gráfico (adotamos isócronas de composição solar dos modelos de PADOVA, conforme detalhes na próxima seção). Essas isócronas são também usadas para a determinação das idades dos aglomerados, escolhendo-se o melhor ajuste aos dados. 


\section{$3.2 \quad \mathrm{O}$ projeto $2 M A S S$ e as Isócronas de Padova}

Em junho de 1997 começava a operar o mais recente projeto de deteç̧ão do céu na região das bandas do infravermelho, o $2 M A S S .{ }^{3}$ Ele foi desenvolvido como um projeto conjunto da University of Massachussetts e do Centro de Processamento e Análise em infravermelho do California Institute of Technology. O 2MASS se estabelece com o intuito de preencher a lacuna existente entre o conhecimento disponível a respeito do céu nas regiões do infravermelho e as tecnologias disponíveis para realizar essa tarefa. Graças ao avanço da tecnologia na detecção nas bandas do infravermelho, ele tem providenciado respostas imediatas para diversas questões acerca da estrutura da Galáxia e do Universo local, como também um novo cenário na interpretação de dados substituindo seu antecessor, o projeto TMSS (The Two Micron Sky Survey [29]).

O 2MASS escaneou de forma homogênea todo o céu em três bandas do infravermelho próximo, a fim de detectar e caracterizar fontes puntuais mais brilhantes que 1 mJy (mili Jansen) em cada banda. Isso significa uma implementação de aproximadamente 80.000 vezes em sensibilidade em relação aos projetos anteriores. O sistema fotométrico compila o Johnson $\mathrm{J}(1.25 \mu \mathrm{m})$ e $\mathrm{H}(1.65 \mu \mathrm{m})$ que são as bandas utilizadas adicionando a banda $K_{s}(2.17 \mu \mathrm{m})$, onde $K_{s}$ é o filtro ' $K$-short' que exclui comprimentos de onda maiores que $2.31 \mu \mathrm{m}$.

Os benefícios imediatos do 2MASS incluem entre outros uma visão sem precedentes da nossa Galáxia quase livre de efeitos de nuvens de poeira interestelar, promover um censo entre as galáxias incluindo resultados fotométricos em três comprimentos de onda e uma base estatística para a procura de objetos astrofísicos raros mas importantes, que são frios e extremamente vermelhos (estrelas de muito baixa luminosidade e anãs marrons) ou objetos obscurecidos em comprimentos de onda visíveis. O $2 M A S S$ tem contribuído fortemente na geração de um Atlas do céu, compilando aproximadamente 4 milhões de imagens de alta resolução, além de dois catálogos. Um

\footnotetext{
${ }^{3}$ Disponível em http://www.ipac.caltech.edu/2mass/releases/allsky/
} 
catálogo contendo informações precisas sobre posições e fluxos para aproximadamente 300 milhões de estrelas e outros objetos não resolvidos, e outro abrangendo mais de um milhão de galáxias e outras nebulosas.

Também são de suma importância em nosso trabalho as isócronas de Padova ${ }^{4}$. As isócronas são ferramentas excelentes para derivar parâmetros como idades, metalicidades função de massa e distâncias a sistemas de estrelas e por isso elas tem uma importância crucial em nosso trabalho. Recentemente Bonatto et. al. [33] propuseram uma comparação entre as isócronas existentes no sistema Johnson e as isócronas geradas a partir do $2 M A S S$ com $\mathrm{J}, \mathrm{H}$ e $K_{s}$. Bonatto et. al. constataram que os parâmetros das isócronas de Padova são os mais próximos à idade e metalicidade WEBDA além de serem mais convenientes para o estudo de aglomerados abertos com metalicidade próxima da solar. Por esse motivo as isócronas de Padova são adotadas em nosso trabalho em todas as determinações de distâncias e idades.

\subsection{Cálculo de distâncias e idades : O Método}

Através do método descrito na seção anterior pudemos desenvolver um programa em linguagem Java capaz de efetuar os cálculos para valores de distância (equação 3.7) e excesso de cor (equações 3.1 e 3.2 ) para cada estrela individualmente. Os resultados de saída do programa são usados como valores preliminares no ajuste de isócronas à sequência principal de cada aglomerado através do método do main sequence fitting.

O programa encontra todos os membros com TE identificados no SIMBAD e com probabilidade de pertinência maior que 51\%. Para tal, ele compara com uma precisão de 0.5 segundo de arco as posições $\alpha$ e $\delta$ J2000 de cada estrela, com as posições fornecidas no SIMBAD. O uso do programa para o cálculo desses parâmetros está

\footnotetext{
${ }^{4}$ As isócronas utilizadas nesse trabalho apresentam-se disponíveis em: http://pleiadi.pd.astro.it
} 
limitado ao fato de encontrar estrelas membro com TE conhecido no SIMBAD. De posse dos TE's fazemos uma interpolação com Tabelas que relacionam cores intrínsecas, magnitudes absolutas aos TE's nas bandas do visível $B, V$ e infravermelho $J, H$ (ver $[25],[26])$.

Com essas informações o programa efetua os cálculos de distância e excesso de cor nas bandas do infravermelho $J$ e $H$ e também nas bandas do visível $B$ e $V$. É muito importante ressaltar que quanto à classe de luminosidade ${ }^{5}$ (denotaremos $L C$ ) das estrelas com TE identificado no SIMBAD, utilizamos em nossos cálculos de distância apenas estrelas com $L C$ tipo $\mathrm{V}$ (anãs). Estrelas com $T E$ peculiares são descartadas (ex: $M 8 e, F 3 I V / V \ldots)$.

O programa efetua os cálculos de distância e excesso de cor para o aglomerado usando dois tipos de dados:

1. Usando dados na freqüência do visível $(B, V)$;

2. Usando dados na freqüência do infravermelho $(J, H)$.

Os resultados de saída do programa nas bandas do visível são os valores de $E(B-V)_{v i s}$ e distância no visível (denotamos $d_{v i s}$ ) calculados para cada estrela individualmente, só depois é tomada a média para o aglomerado. Para obter o valor de $<E(J-H)_{v i s}>$ basta converter o valor de $<E(B-V)_{\text {vis }}>$ a partir da equação 3.4. Assim, a Equação 3.3 para o avermelhamento fica totalmente obtida com dados na frequência do visível. A distância é obtida através da Equação 3.7.

O mesmo procedimento é adotado nas bandas do infravermelho, com a diferença de que são usados dados do Catálogo 2MASS, e as Equações 3.1 , 3.6. Para o cálculo do valor da distância é usada a equação 3.7, sendo que os resultados de saída são portanto $<$ dist $_{\text {inf }}>$, distância calculada no infravermelho e $<E(J-H)_{\text {inf }}>$, excesso de cor calculado no infravermelho, onde o subscrito “inf” denota que os cálculos foram

\footnotetext{
${ }^{5}$ Do inglês Luminosity Class
} 
efetuados usando apenas dados nas bandas do infravermelho próximo, e o subscrito "vis" denota que os cálculos foram efetuados apenas nas bandas do visível .

É importante ressaltar que o programa calcula os valores de distância, excessos de cor e avermelhamento para cada estrela, sendo que só depois é tomado o valor médio para o aglomerado. Os resultados de saída do programa são portanto $<d_{i s t} t_{\text {inf }}>$, $<E(J-H)_{\text {inf }}>,<$ dist $_{\text {vis }}>\mathrm{e}<E(J-H)_{\text {vis }}>$.

Depois de calculadas distâncias e excessos de cor preliminares são utilizados para o ajuste de isócronas à sequência principal dos algomerados. Primeiro ajustamos a Sequência Principal de Idade Zero ${ }^{6}$ à sequência principal do nosso aglomerado com os valores preliminares, a diferença entre a magnitude aparente das estrelas do nosso aglomerado e Magnitudes absolutas da curva da sequência principal teórica que ajustamos, chamamos módulo de distância, que é dado de acordo com a equação 3.7. Depois de ajustar a sequência principal de idade zero, ajustamos uma outra isócrona para encontrar a idade. Damos maior peso à presença de estrelas gigantes e ao ponto de virada da sequência principal.

\footnotetext{
${ }^{6}$ No inglês denominada ZAMS de zero age main sequence
} 


\section{Capítulo 4}

\section{Resultados - Parâmetros Cinemáticos}

Apresentamos neste Capítulo a análise e discussão dos resultados obtidos acerca de movimentos próprios e probabilidades de pertinência para os aglomerados abertos estudados neste projeto.

Para 247 aglomerados trata-se do primeiro resultado de movimentos próprios obtido, e para os casos que apresentam soluções já catalogadas na literatura, apresentamos além das soluções, comparações com os resultados disponíveis (especificamente DAML02 e Kharchenko et. al. [30]). No final do Capítulo fazemos uma breve discussão dos casos para os quais não foi possível obter solução.

\subsection{Resultados de Movimentos Próprios e probabili- dades de pertinência para os aglomerados estuda- $\operatorname{dos}$}

A primeira etapa do projeto consistiu inicialmente na seleção de uma amostra de aglomerados abertos listados na versão 2.3 do catálogo de Dias et. al.. Foram selecionados 852 objetos nunca antes estudados do ponto de vista cinemático ou distâncias determiandas, portanto não possuíam na literatura resultados disponíveis para 
comparação. Dando início às análises constatamos que 87 aglomerados não poderiam ter estrelas extraídas do catálogo UCAC2 uma vez que os aglomerados não apresentavam posições dentro do limite de declinação do catálogo ${ }^{1}$. Descartando esses casos a nossa amostra fica constituída por 765 aglomerados.

Foram analisados os movimentos próprios médios e probabilidades de pertinência das estrelas na região dos aglomerados em questão, sendo que nem todos apresentaram solução satisfatória. Os resultados podem ser resumidos da seguinte forma:

- Aglomerados que possuem solução satisfatória $319(41 \%)$

- Aglomerados que possuem solução com sigma muito alto ou divergem......266(35\%)

- O programa de busca VIZIER encontrou poucas estrelas ou não encontrou estrelas UCAC2 em torno da posição procurada $.150(20 \%)$

- Raio do aglomerado muito grande ou muito pequeno $.30(4 \%)$

Consideramos como satisfatória ${ }^{2}$ a solução que apresente: convergência na solução do sistema de equações não lineares ${ }^{3}$; valor de $\sigma$ em torno de 3 mas/yr; boa distribuição de probabilidades de pertinência (duas populaçõs bem separadas) ; um diagrama VPD onde seja possível distinguir as duas populações: membros e intrusas .

As regiões do céu analisadas para os 319 aglomerados abertos, cujas soluções foram obtidas, envolvem 83.563 estrelas, das quais 19.967 foram consideradas como membros astrométricos dos aglomerados e os resultados obtidos estão disponíveis na Tabela A em anexo no final do texto da dissertação.

A seguir prosseguiremos com as comparações dos nossos resultados com os resultados catalogados na literatura.

\footnotetext{
${ }^{1}$ Os limites de declinação do catálogo UCAC2 foram devidamente mencionados na seção 2.3.

${ }^{2}$ Uma solução satisfatória pode ser considerada dentro dos padrões descritos na seção 2.3

${ }^{3}$ Lembramos aqui que os cálculos de movimentos próprios são efetuados conforme descrito na seção 2.2
} 


\subsubsection{Comparações com resultados obtidos na literatura}

Alguns aglomerados da nossa amostra possuem publicados na literatura resultados de movimentos próprios médios. Ao se iniciar o projeto os aglomerados selecionados não apresentavam resultados disponíveis na literatura, mas durante o período de seu desenvolvimento foram publicados alguns resultados de parâmetros cinemáticos e fundamentais para vários objetos da nossa amostra. Os resultados foram fornecidos por Kharchenko et. al. [30].

Fazemos nas subseções 4.1.2 e 4.1.3 uma comparação dos nossos valores de movimentos próprios com resultados disponíveis na literatura (especificamente DAML02 e Kharchenko et. al. [30]). Lembramos que a comparação mostrada nas seções seguintes não é a mesma mostrada na seção 2.4 onde tratávamos da confiabilidade do método por nós empregado na determinação de movimentos próprios.

\subsubsection{Comparação com os resultados disponíveis no DAML02}

Dos 319 casos com solução satisfatória obtidos em nosso trabalho, 72 constam na versão 2.6 do catálogo DAML02. Os resultados obtidos por nós como os resultados catalogados no DAML02 estão sumarizados na Tabela B em anexo no final do texto da dissertação.

A seguir fazemos uma comparação dos resultados nas figuras 4.1 e 4.2 . Os gráficos exibem a comparação nas coordenadas $(\mu \alpha \cos \delta$ e $\mu \delta)$ entre os nossos resultados e os resultados publicados no DAML02.

Comparamos as duas amostras através do ajuste de uma gaussiana aos histogramas das diferenças em cada coordenada que forneceram uma média nas diferenças igual a $-0,31$ mas/yr para $\mu \alpha \cos \delta$ com desvio padrão de 1,92 e -0,55mas/yr para $\mu \delta$ com desvio padrão de 2,27 . 

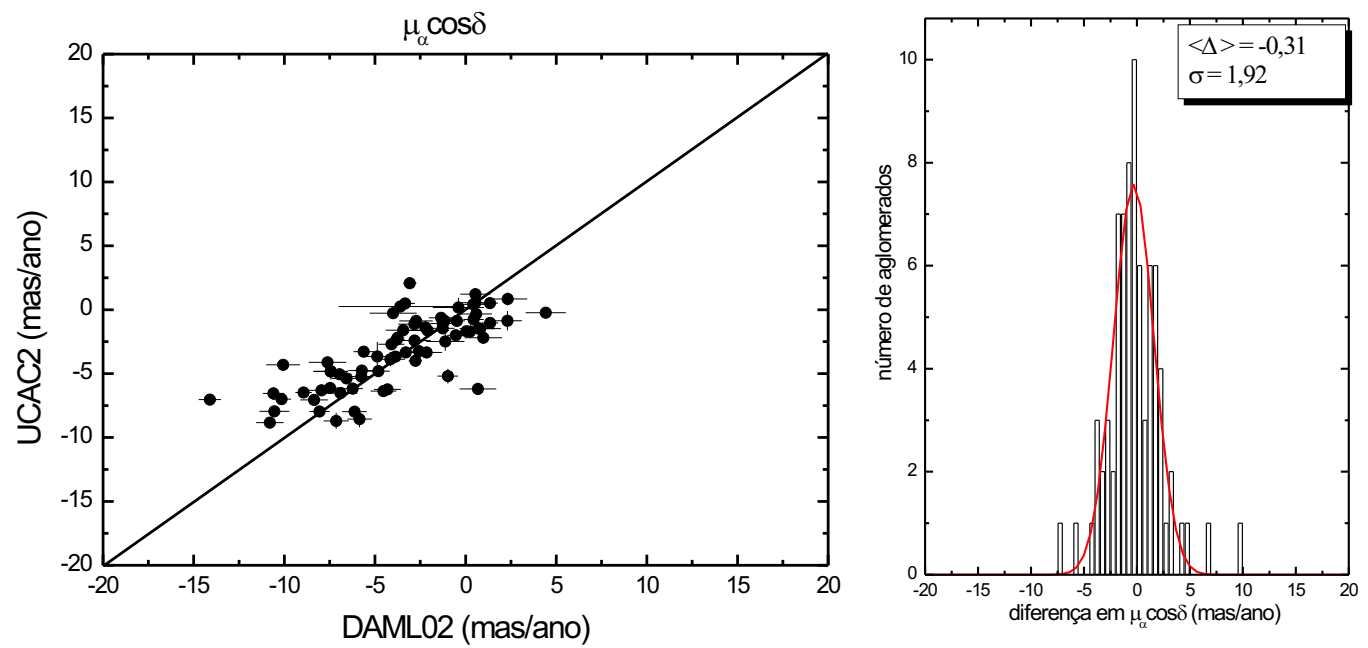

Figura 4.1: Apresentamos a comparação dos movimentos próprios médios obtidos neste trabalho na coordenada $\mu \alpha \cos \delta$ com outros disponíveis na literatura compilados no nosso catálogo de aglomerados abertos (DAML02). No painel à esquerda não temos um ajuste, mas sim a reta de $45^{\circ}$ que representa regiões de movimento próprio idêntico. O painel à direita representa a distribuição das diferenças em movimentos próprios médios e os símbolos $\langle\Delta\rangle$ e $\sigma$ denotam respectivamente o valor médio das diferenças na coordenada $\mu \alpha \cos \delta$ e o valor do desvio padrão.
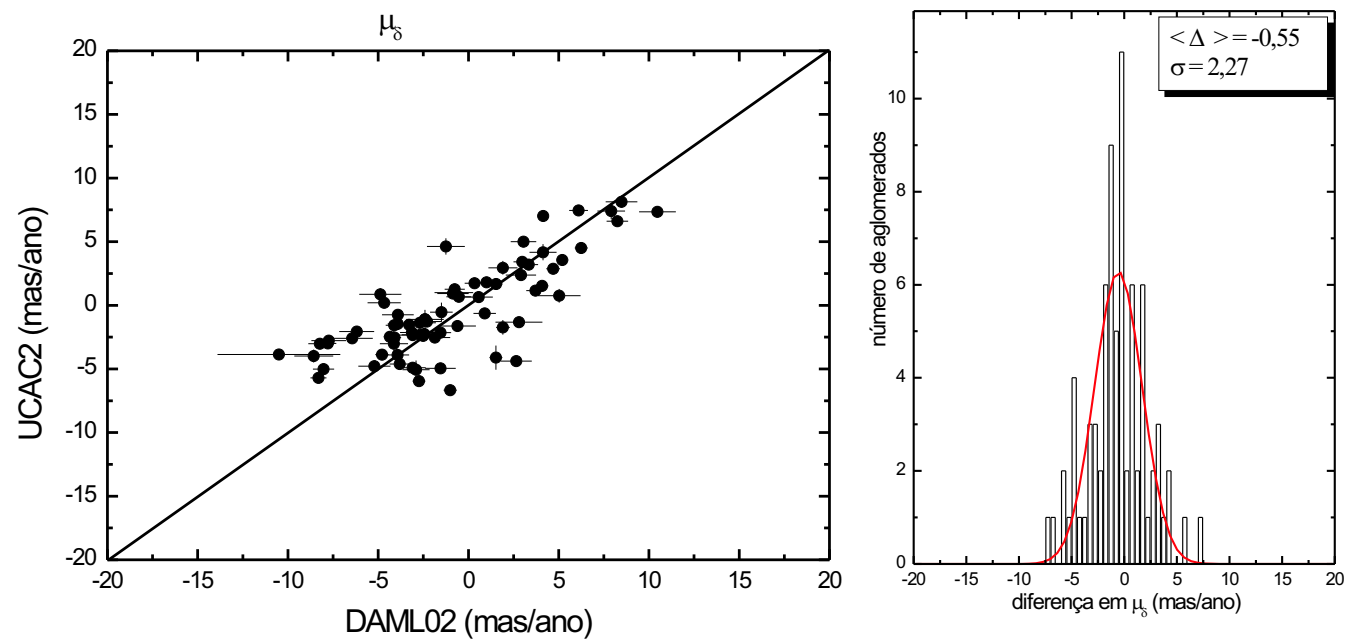

Figura 4.2: Apresentamos a comparação dos movimentos próprios médios obtidos neste trabalho na coordenada $\mu \delta$ com outros disponíveis na literatura compilados no nosso catálogo de aglomerados abertos (DAML02). No painel à esquerda não temos um ajuste, mas sim a reta de $45^{\circ}$ que representa regiões de movimento próprio idêntico. O painel à direita representa a distribuição das diferenças em movimentos próprios médios e os símbolos $\langle\Delta\rangle$ e $\sigma$ denotam respectivamente o valor médio das diferenças na coordenada $\mu \delta$ e o valor do desvio padrão.

As comparações mostram que as diferenças encontradas estão dentro da 
precisão de nossas medidas ${ }^{4}$. A média das diferenças mostra que não há tendências sistemáticas e o desvio padrão assegura que os dois conjuntos de valores estão em bom acordo. Alguns dos casos discrepantes serão discutidos posteriormente.

Apresentamos a última comparação entre os resultados UCAC2 e DAML02 no diagrama que mostra as diferenças em $\mu \alpha \cos \delta$ e $\mu \delta$ simultaneamente.

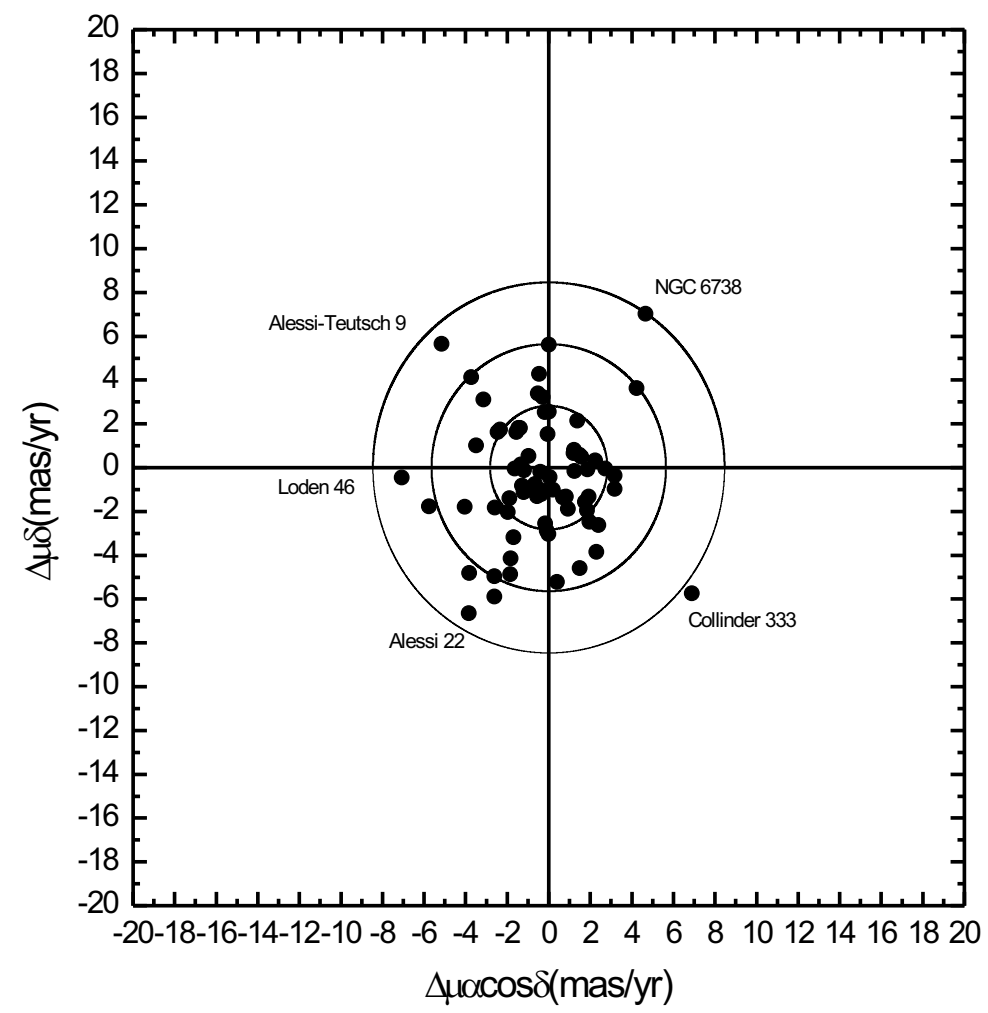

Figura 4.3: O gráfico apresenta diferenças em movimento próprio médio nas coordenadas $\mu \alpha \cos \delta$ e $\mu \delta$. Os círculos mostram as regiões de desvio padrão nas coordenadas, sendo iguais a 1, 2 e 3 vezes o valor de $\sigma$. Alguns dos aglomerados fora do raio de $3 \sigma$ são discutidos no texto.

De acordo com a figura 4.3 nenhum aglomerado está fora da margem de 3 vezes o desvio padrão dos resultados de movimentos próprios nas coordenadas $\mu \alpha \cos \delta$ e $\mu \delta$ à exceção do aglomerado Collinder 333.

Os pontos na região entre $2 \sigma$ e $3 \sigma$ ( 7 aglomerados) correspondem a $11 \%$

\footnotetext{
${ }^{4}$ Exploramos a precisão de nossas medidas na seção 2.4 ao comparar nossos resultados obtidos com o catálogo UCAC2 com outros resultados provenientes do catálogo Tycho2 [24].
} 
da amostra comparada. Os pontos relativos aos aglomerados: Alessi-Teutsch 9, Alessi 22, Loden 46 , NGC 6738 e Collinder 333, podem ser observados em todos os gráficos anteriormente apresentados como os pontos mais discrepantes em relação à amostra de resultados comparada.

No caso dos aglomerados Alessi-Teutsch 9 , Loden 46 e Alessi 22 que possuem um diâmetro aparente muito grande, nossa análise não foi capaz de distinguir membros e estrelas intrusas, e com isso obter uma boa determinação de movimentos próprios médios. Isso se deve ao fato dos aglomerados serem muito grandes ${ }^{5}$ e possuírem muitas estrelas no campo de observação. Como foram muitas estrelas consideradas como membros, isso aumenta a chance de ruído inserido na amostra, o que prejudica a determinação de parâmetros cinemáticos (movimento próprio médio e determinação dos membros do aglomerado). A figura 4.4 traz os CMD's e VPD's para Alessi-Teutsch 9 , Alessi 22 e Loden 46.

Como se pode perceber pelos diagramas da figura 4.4, os aglomerados Alessi-Teutsch 9, Alessi 22 e Loden 46 possuem muitas estrelas no campo, o que impede uma boa determinação de movimentos próprios.

Para os aglomerados NGC 6738 e Collinder 333, a diferença na determinação dos movimentos próprios pode ser justificada pelo número de estrelas empregadas no cálculo; o número encontrado por nós é maior que o número de estrelas usadas pelo autor do DAML02 para fazer o cálculo de movimentos próprios. Para Collinder 333 são consideradas 30 estrelas contra apenas 4 estrelas encontradas no DAML02. Para NGC 6738 são consideradas 51 estrelas contra apenas 4 estrelas encontradas no DAML02. Valores de erro encontrados em cada coordenada na literatura para esses aglomerados também são maiores que os valores determinados em nossa análise.

\footnotetext{
${ }^{5}$ Referimo-nos aqui ao diâmetro aparente dos objetos
} 

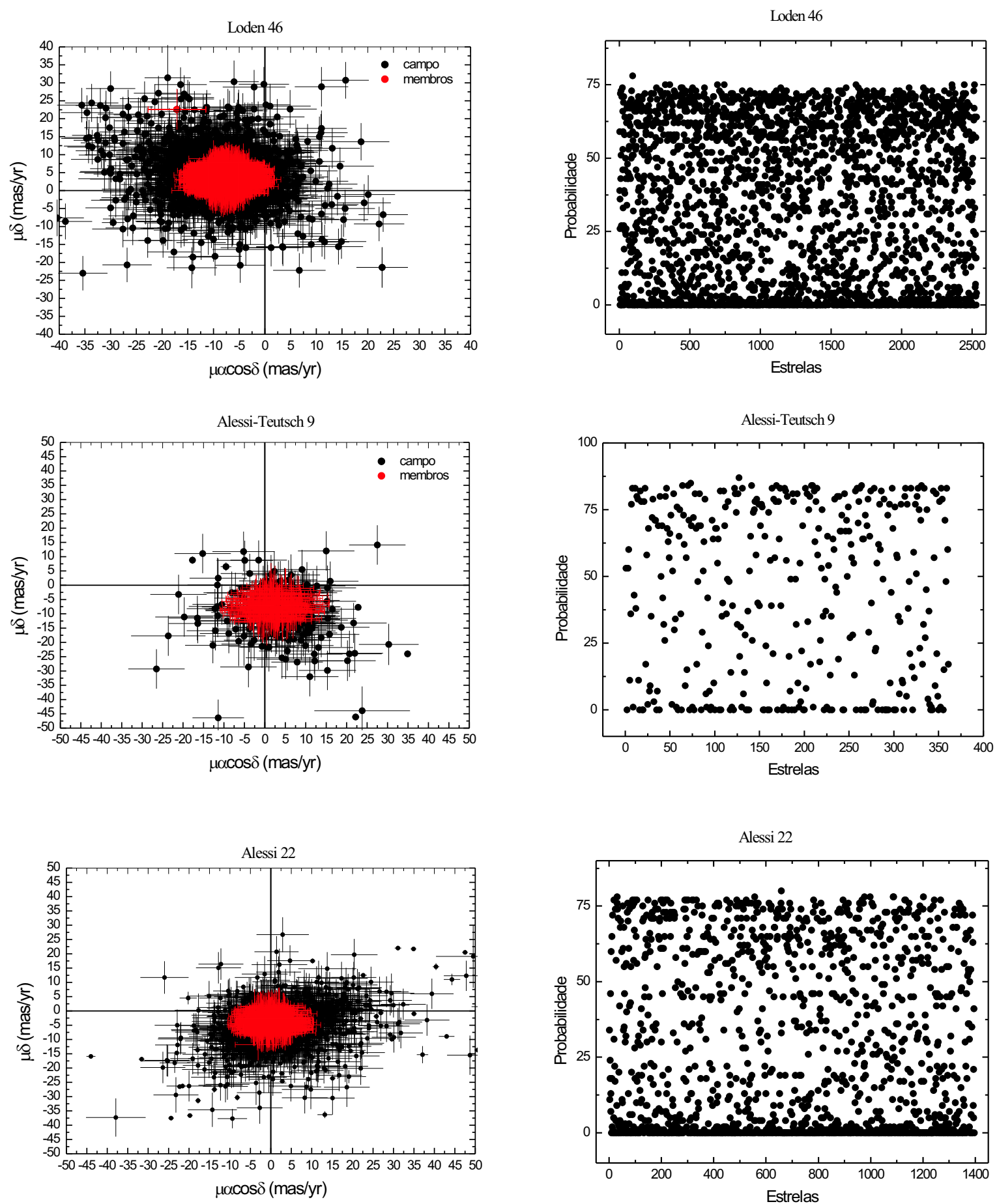

Figura 4.4: Diagramas VPD e de probabilidades para os aglomerados Alessi-Teutsch 9, Alessi 22 e Loden 46. Percebemos em todos os casos a existência de muitas estrelas no campo de observação. Consideramos como membros as estrelas que possuem uma probabilidade de pertinência maior que $51 \%$. 

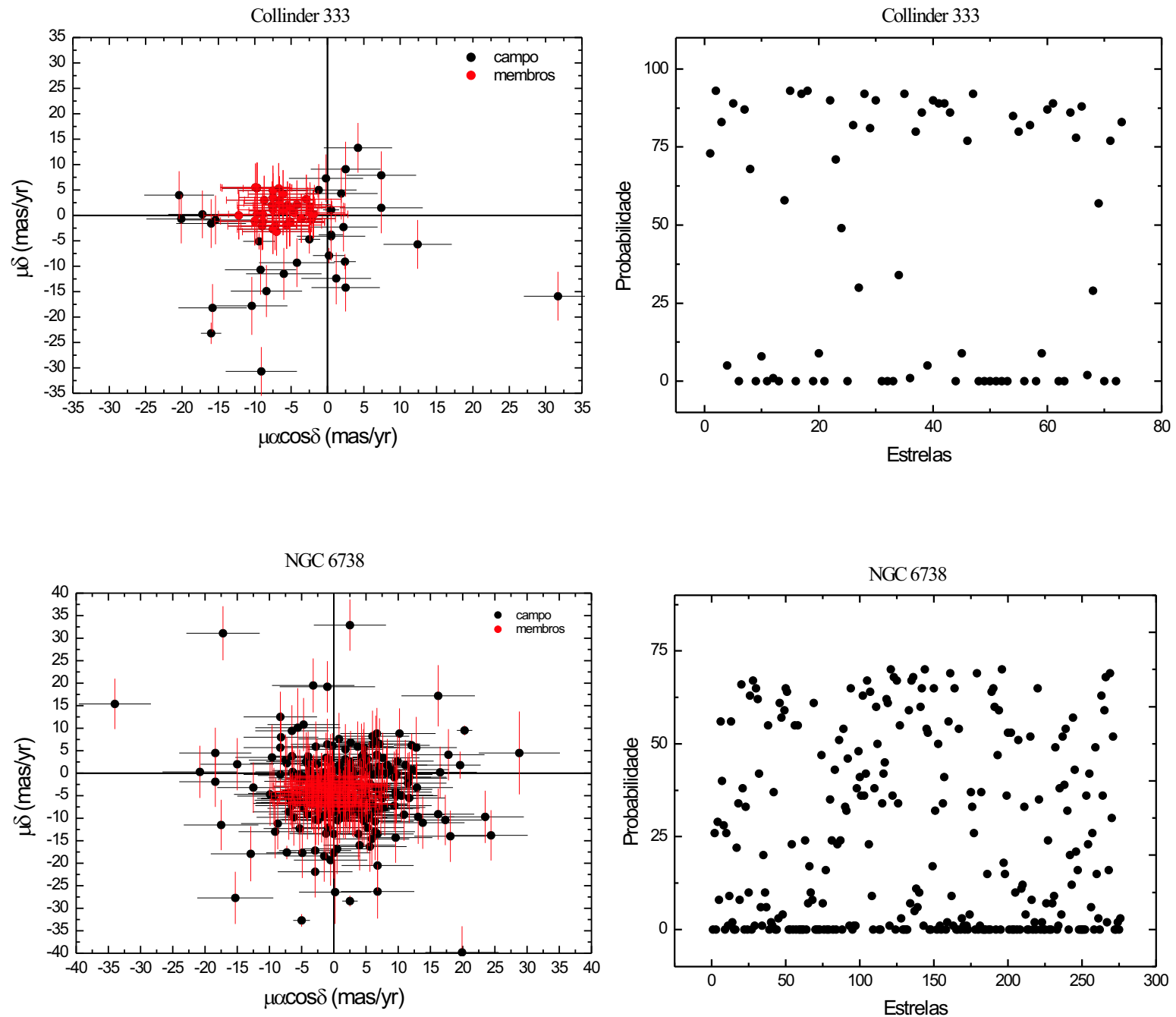

Figura 4.5: Diagramas VPD para os aglomerados NGC 6738 e Collinder 333, e diagramas de probabilidades de pertinência para as estrelas do aglomerado. Os diagramas mostram a boa separação entre a população de membros e intrusas no campo do aglomerado. Lembramos que consideramos como membros as estrelas que possuem uma probabilidade de pertinência maior que $51 \%$, no diagrama VPD os membros estão representados pela cor vermelha.

De acordo com a figura 4.5 nos diagramas VPD, percebemos duas populações bem separadas: membros do aglomerado (Probabilidades de pertinência maiores que 51\%) e estrelas intrusas (probabilidades de pertinência menores que 51\%). Como podemos ver nos diagramas de probabilidades existem duas populações distintas, uma com probabilidades de pertinência altas e outra com probabilidades de pertinências próximas ao zero, nos dois casos. 


\subsubsection{Comparação com os resultados fornecidos por Kharchenko}

\section{et. al. 2005}

Fazemos nesta seção uma comparação dos nossos resultados com os resultados obtidos por Kharchenko et. al. [30] ${ }^{6}$.

Nas figuras 4.6 e 4.7 a seguir apresentamos as comparações dos valores de movimentos próprios em cada coordenada $(\mu \alpha \cos \delta$ e $\mu \delta)$ para 48 aglomerados que possuem resultados calculados pelo nosso grupo e por K05.
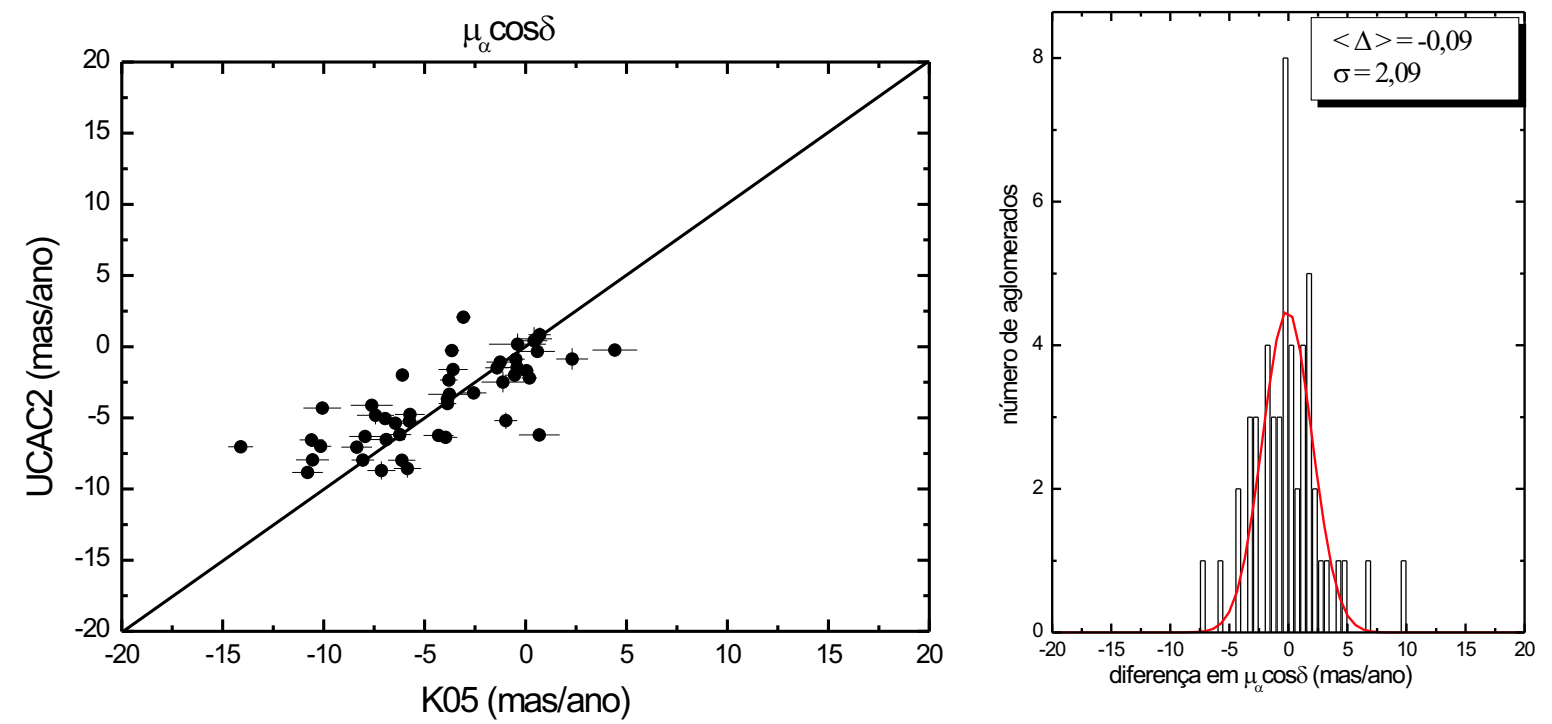

Figura 4.6: Comparação do movimento próprio médio em $\mu \alpha \cos \delta$ obtidos neste trabalho com os valores fornecidos por K05 compilados no nosso catálogo de aglomerados abertos (DAML02). No painel da esquerda a reta de $45^{\circ}$ representa regiões de movimento próprio idêntico. O painel à direita mostra a distribuição das diferenças em movimentos próprios e os símbolos $\langle\Delta>$ e $\sigma$ denotam respectivamente o valor médio das diferenças na coordenada $\mu \alpha \cos \delta$ e o valor do desvio padrão.

Comparamos as duas amostras através do ajuste de uma gaussiana aos histogramas das diferenças em cada coordenada que forneceram uma média igual a $-0,09$ mas/yr na coordenada $\mu \alpha \cos \delta$ e 2,09 para o valor do desvio padrão. $\mathrm{Na}$ coordenada $\mu \delta$ temos que a média das diferenças entre as duas amostras é igual a $-0,57$ mas/yr, sendo que o desvio padrão é 2,25 .

\footnotetext{
${ }^{6}$ Daqui em diante denominaremos K05
} 

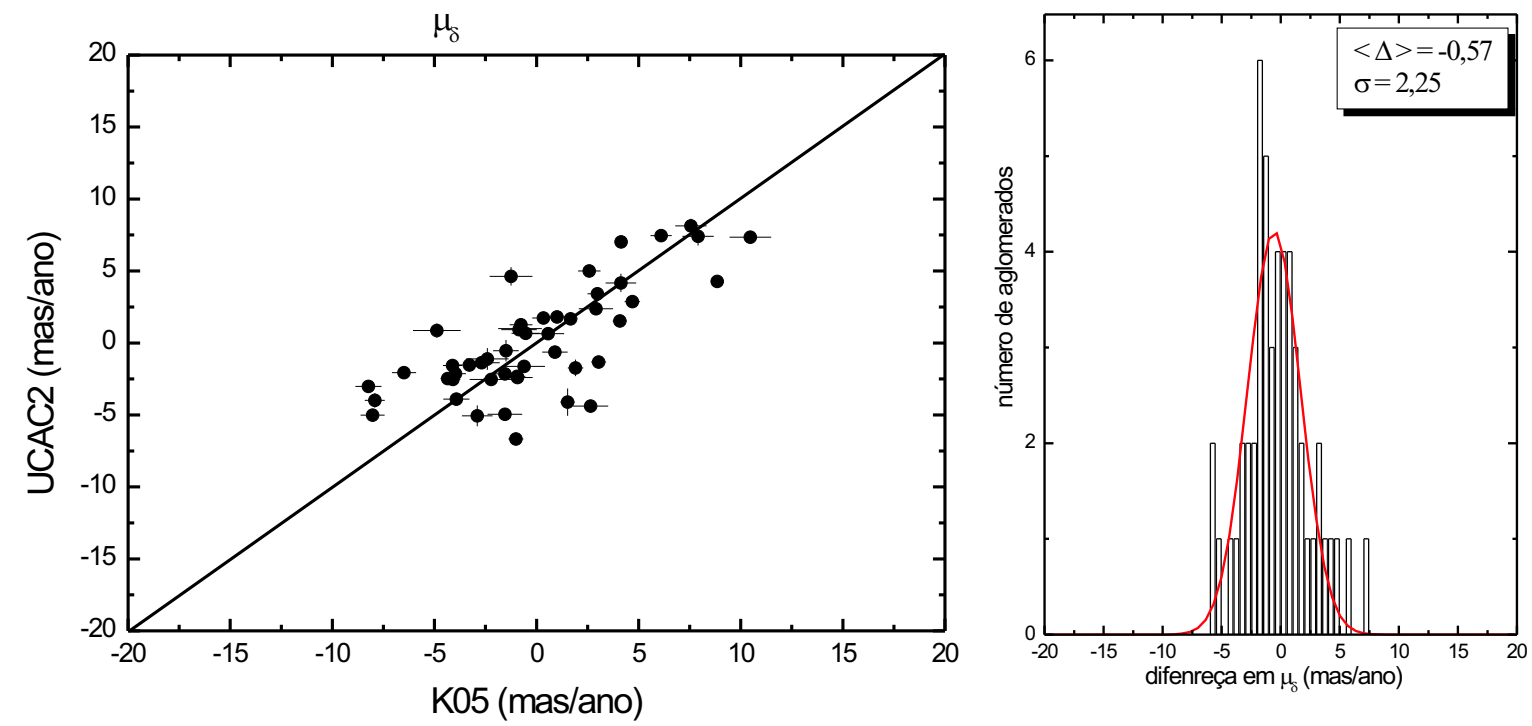

Figura 4.7: Comparação do movimento próprio na coordenada $\mu \delta$ obtidos neste trabalho com os obtidos por K05. A reta na figura do painel à esquerda não é um ajuste, a reta de $45^{\circ}$ representa regiões de movimento próprio idêntico. No painel à direita temos a distribuição das diferenças em movimentos próprios e os símbolos $<\Delta>$ e $\sigma$ denotam respectivamente o valor médio das diferenças na coordenada $\mu \delta$ e o valor do desvio padrão.

As comparações mostram que as diferenças encontradas estão dentro da precisão de nossas medidas. A média das diferenças mostra que não há tendências sistemáticas e o desvio padrão assegura que os dois conjuntos de valores estão em bom acordo, à exceção de alguns casos que serão discutidos.

São comparadas as diferenças entre nossos resultados e Kh05 em movimentos próprios nas duas coordenadas $\Delta \mu \alpha \cos \delta$ e $\Delta \mu \delta$. De acordo com a figura 4.8, nenhum aglomerado está fora da margem de 3 vezes o desvio padrão dos resultados de movimentos próprios nas coordenadas $\mu \alpha \cos \delta$ e $\mu \delta$. Até mesmo o aglomerado Collinder 333, que na comparação tratada na seção 4.1 .2 mostrava-se fora dessa margem de $3 \sigma$. Na seção 4.1.2 são discutidos os casos discrepantes Collinder 333, NGC 6738 , Loden 46 e Alessi-Teutsch 9. Todos esses casos tiveram seus movimentos próprios determinados por Kh05, e portanto uma discussão desses aglomerados pode ser acompanhada na seção mencionada. 


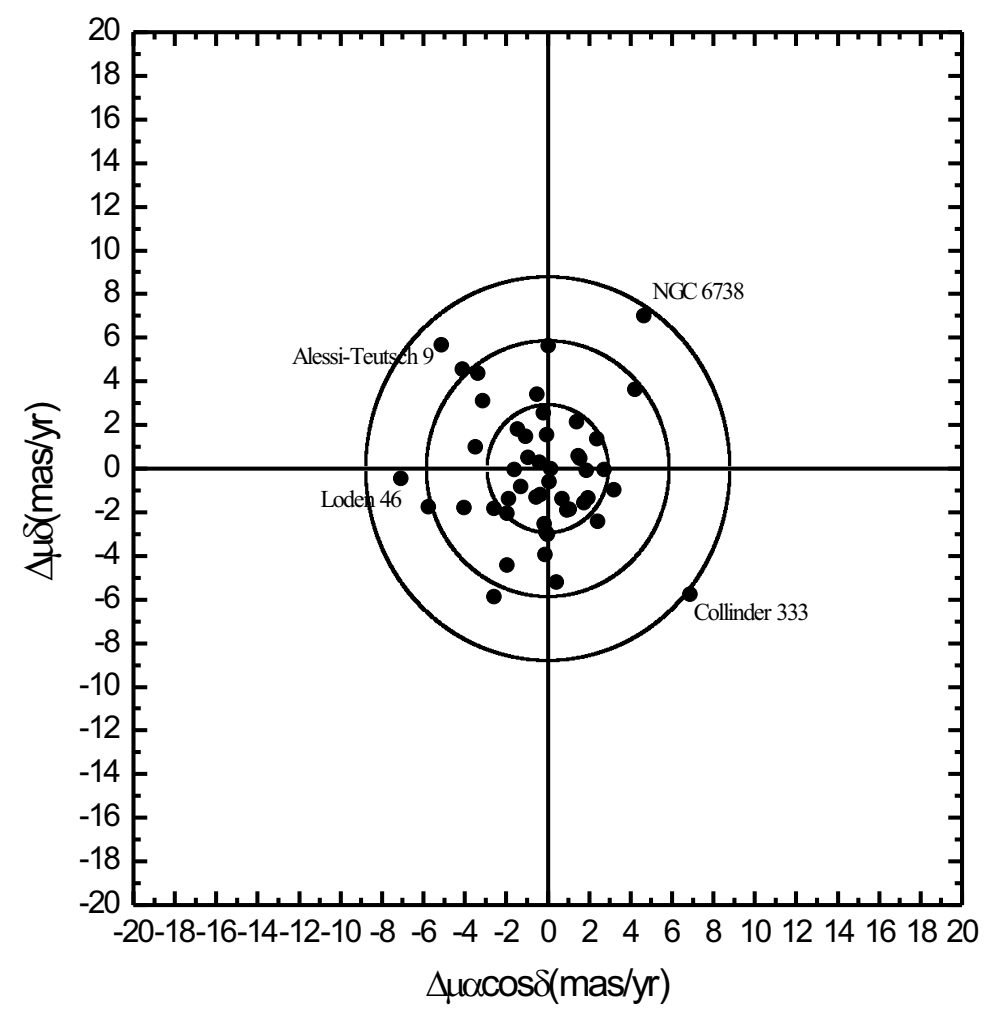

Figura 4.8: O gráfico apresenta diferenças em movimento próprio médio nas coordenadas $\mu \alpha \cos \delta$ e $\mu \delta$. Os círculos mostram as regiões de desvio padrão nas coordenadas, sendo iguais a 1,2 e 3 vezes o valor de $\sigma$. Os aglomerados fora do raio de $3 \sigma$ são os mesmo aglomerados dicutidos na comparação inserida na seção 4.1.2.

Os pontos na região entre $2 \sigma$ e $3 \sigma(13$ aglomerados ) correspondem a $27 \%$ da amostra comparada, enquanto que $58 \%$ da amostra encontra-se dentro da região de $1 \sigma$.

\subsubsection{Casos sem solução}

Conforme mencionamos na seção 1.3 do capítulo introdutório da dissertação, faremos aqui uma breve discussão de alguns casos para os quais não apresentamos movimentos próprios e probabilidades de pertinência aos aglomerados analisados.

Para 4\% da nossa amostra, cerca de 30 aglomerados, notamos que os mesmos apresentavam um diâmetro aparente muito grande ou muito pequeno. Podemos 
citar como exemplo de aglomerado que apresenta diâmetro aparente grande o Alessi 33, diâmetro 65 minutos de arco, já os casos que apresentam diâmetro aparente pequeno: ESO 456-72, diâmetro 1.4 e Dutra-Bica 5 , diâmetro 0.4, todos em minutos de arco. Pudemos identificar com a ajuda da ferramenta Vizier estrelas em torno da posição fornecida para extração, porém, o número de estrelas encontrado ou foi insuficiente, ou o campo era demasiado grande (apresentava muitas estrelas) para proceder com a análise proposta na seção 2.2. Tipicamente um campo com poucas estrelas possui da orden de dez ou menos estrelas identificadas pelo Vizier, já um campo que apresenta muitas estrelas possui cerca de duas mil ou mais estrelas.

Em outros 150 casos, 20\% da amostra, foram encontradas poucas estrelas ou nenhuma estrela UCAC2 em torno da posição procurada. Nesses últimos casos mencionados os aglomerados não apresentavam necessariamente um diâmetro aparente muito pequeno ou muito grande. Ressaltamos que estamos apresentando apenas alguns exemplos de aglomerados com poucas estrelas no campo analisado, e que foram tentados mais de um raio para a extração das mesmas sem sucesso, ou seja, o número de estrelas presentes no campo de observação continuava muito pequeno para se aplicar o método estatístico de Zhao \& He [8].

Para 266 casos, não apresentamos uma solução satisfatória de acordo com o método utilizado (as soluções possuem valor de $\sigma$ muito alto ou simplesmente não apresentaram uma solução convergente ${ }^{7}$ ). Esses aglomerados representam $35 \%$ da amostra de aglomerados que pretendíamos tratar no início do projeto. Isso ocorre em alguns casos pelo grande número de estrelas encontradas em torno da posição fornecida para

\footnotetext{
${ }^{7}$ Aqui lembramos a seção 2.2: "Valores iniciais aproximados são fornecidos para todos os parâmetros, e as equações iteradas uma de cada vez, variando o parâmetro associado até que o valor que melhor satisfaça a equação seja obtido. Este valor então substitui seu valor inicial e o processo se repete para a próxima equação onde um valor melhor para o parâmetro associado é encontrado e assim por diante. Quando o processo progrediu de forma que as oito equações tenham sido iteradas sem nenhuma mudança nos valores dos parâmetros, esses são por sua vez adotados como os valores que melhor se ajustam à distribuição de acordo com o modelo adotado". Consideramos que o sistema é não convergente quando não foi atingido durante a iteração um valor estável para os parâmetros (movimentos próprios e sigma).
} 
extração, pelo fato do aglomerado possuir um diâmetro aparente ou muito grande ou muito pequeno e até mesmo por estar muito próximo (distância pequena) o que compromete o uso do nosso programa descrito na seção 2.3. Citamos os casos onde ocorre a não convergência da solução: ESO 493-03, ESO 429-13, Herschel 1 e ESO 245-09.

Ressaltamos, por último, o caso do aglomerado ESO 245-09 para o qual obtivemos uma solução convergente, porém, com um valor de $\sigma$ muito alto, o que torna a solução pouco confiável. No caso desse último aglomerado pudemos verificar sua classificação no catálogo DAML02 e notamos que ele está classificado como um possível remanescente de aglomerado aberto, de acordo com Bica et. al. [32]. Como podemos perceber pela imagem DSS do aglomerado na figura 4.10, ele é formado por poucas estrelas o que justifica a má determinação de movimentos próprios pelo método empregado 2.3. Na figura 4.9 os gráficos CMD e VPD confirmam os maus resultados para os movimentos próprios do aglomerado. De acordo com o VPD não percebemos uma clara separação entre os vetores movimento próprio das estrelas membros e intrusas.
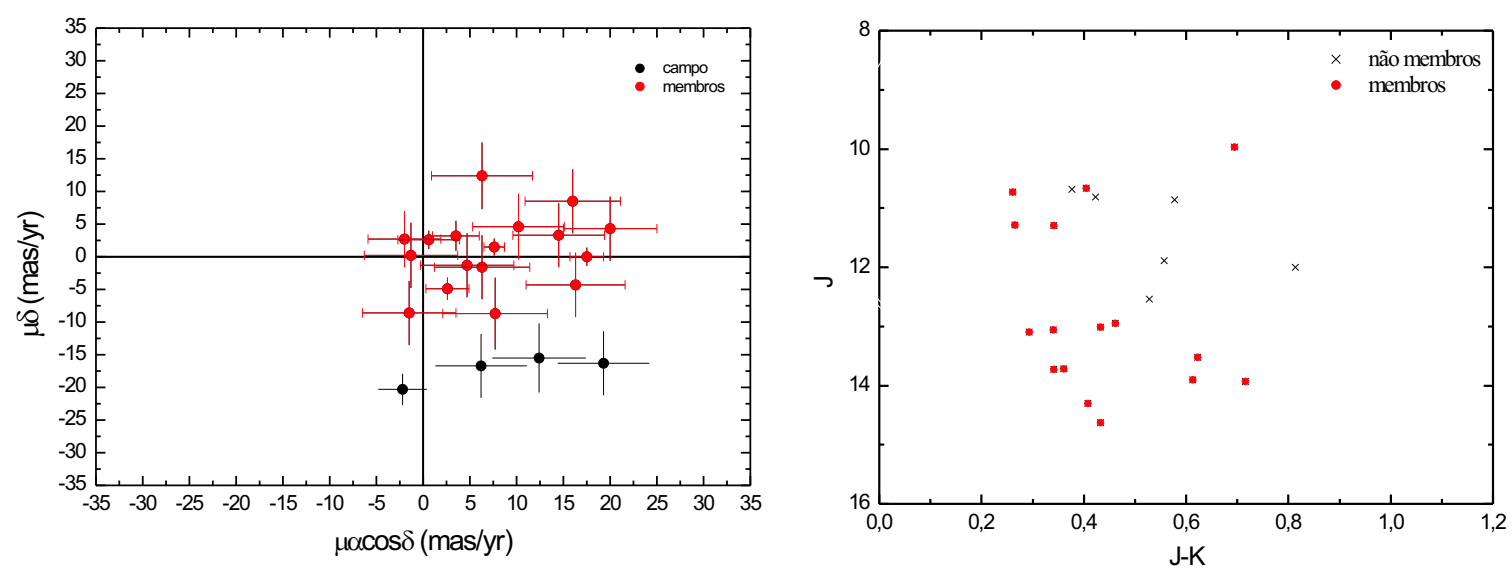

Figura 4.9: Diagramas $V P D$ e $C M D$ para o aglomerado ESO 245-09, ferramenta de análise do programa utilizado para o cálculo de movimentos próprios médios dos aglomerados. Em vermelho as estrelas com probabilidades de pertinência maiores que $50 \%$.

Na figura 4.10 estão as imagens DSS de alguns casos em que os movimentos próprios não foram determinados. 


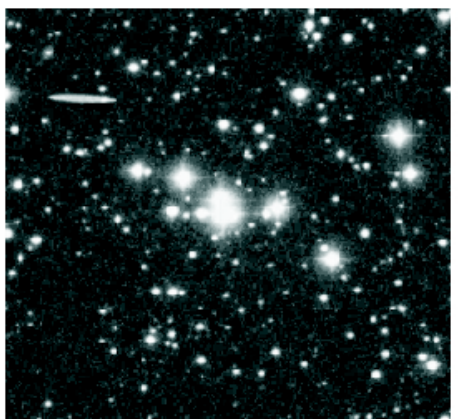

Herschel 1

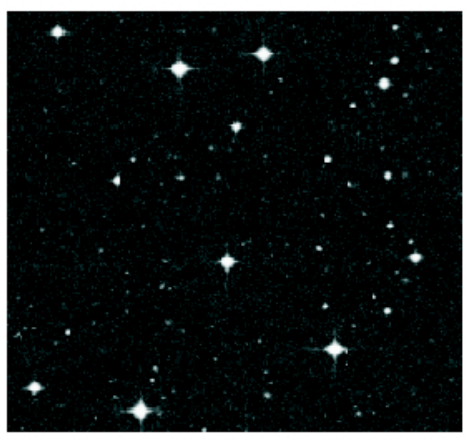

ESO 245-09

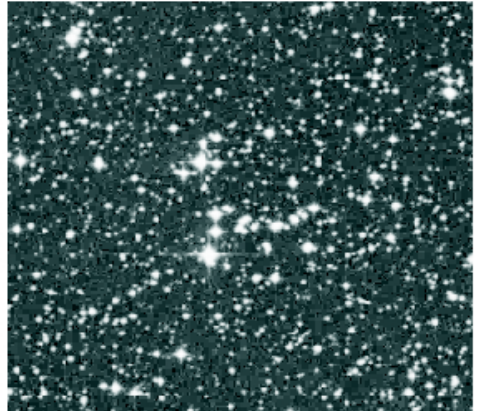

ESO $429-13$

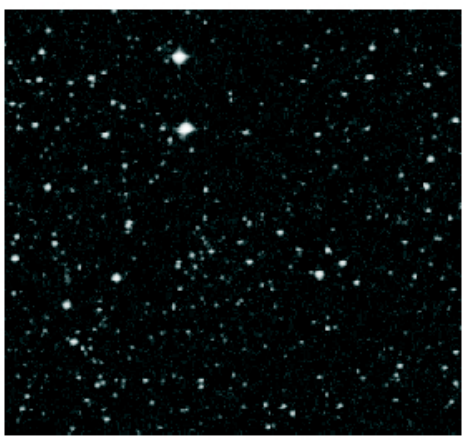

Dutra-Bica 5

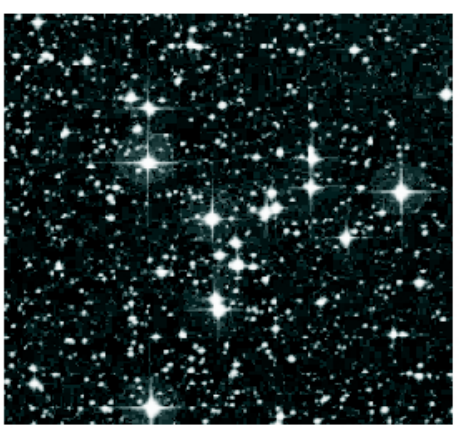

ESO $493-03$

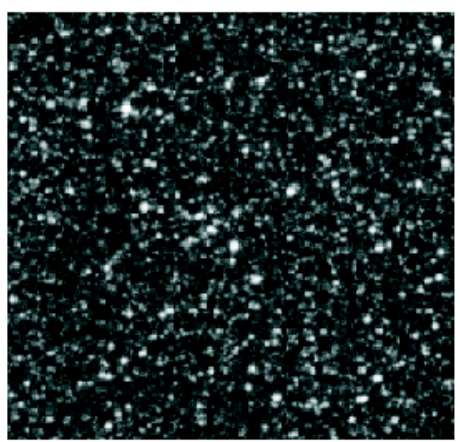

$E S O 456-72$

Figura 4.10: Imagens tiradas do Digitized Sky Survey dos aglomerados cujos movimentos próprios não puderam ser determinados. Os tamanho das imagens dos aglomerados Herschel 1, ESO 429-13, ESO 493-03, ESO 245-09, Dutra-Bica 5 e ESO 456-72 são respectivamente 43.2, 4, 7, 14, 0.4 e 1.4 todos em minutos de arco. Diâmetros e coordenadas foram extraídos do catálogo DAML02.

Determinamos movimentos próprios e probabilidades de pertinência para 319 aglomerados abertos, o que significa um incremento de cerca de $20 \%$ no total de aglomerados abertos catalogados no DAML02 e 41\% da nossa amostra. A etapa seguinte do projeto refere-se a obtenção de parâmetros fundamentais para a amostra inicialmente escolhida de objetos. Para que esses parâmetros possam ser corretamente determinados é preciso inicialmente segregar os membros do aglomerado, entre intrusas e membros. A partir da identificação dos membros dos aglomerados construímos diagramas cormagnitude que nos possibilitaram a determinação de distâncias, excessos de cor e idades. Dessa forma, analisaremos os 319 casos para os quais obtivemos soluções de movimentos próprios e probabilidades de pertinência. 


\section{Capítulo 5}

\section{Resultados - Parâmetros}

\section{Fundamentais}

Apresentamos neste capítulo a análise e discussão dos resultados dos parâmetros fundamentais determinados para os aglomerados abertos que tiveram seus movimentos próprios determinados no Capítulo 4.

Propomos a apresentação dos resultados referentes ao estudo de distâncias, excessos de cor e idades a partir dos diagramas cor-magnitude dos aglomerados conforme o método main sequence fitting descrito na Seção 3.3. Também são apresentadas comparações com resultados disponíveis na literatura e uma breve discussão para os casos em que não obtivemos soluções.

\subsection{Resultados de Parâmetros Fundamentais para os aglomerados estudados}

Para todos os casos com movimentos próprios determinados (319 casos) foram construídos diagramas cor-magnitude a partir das estrelas investigadas anteriormente na parte de determinação de movimentos próprios e probabilidades de perti- 
nência, vide Capítulo 4. Na análise dos diagramas foram consideradas principalmente estrelas com probabilidades maiores que 51\%, o que possibilitou a escolha de quais desses diagramas teriam melhores condições para se fazer uma boa determinação dos parâmetros fundamentais. Ressaltamos que nos baseamos principalmente na observação da forma da sequência principal no diagrama, e com menor peso, se o aglomerado possuía ou não Gigantes presentes na amostra de estrelas presentes no campo.

Foram selecionados 66 casos do total de 319, para determinação dos parâmetros fundamentais, distância, excesso de cor nas bandas do infravermelho e idades. Dos casos selecionados para se fazer essa determinação, 32 apresentam solução satisfatória, $49 \%$ do total, enquanto que os 34 aglomerados restantes não puderam ter suas soluções determinadas ou por não apresentarem membros com TE ${ }^{1}$ identificado no catálogo SIMBAD, ou por apresentarem apenas membros com TE peculiar. Esses casos que não apresentam estrelas membro com TE publicado podem ser analisados mas não faremos tal análise neste trabalho.

Os resultados numéricos para os parâmetros fundamentais podem ser visualizados em tabelas em anexo (Tabelas C, D, E e F), que apresentam não só resultados para os parâmetros fundamentais como também as estrelas usadas no cálculo dos valores preliminares de distância e $E(J-H)^{2}$. Apresentamos também em anexo 7.1 os diagramas CMD nas bandas do infravermelho próximo (bandas $J, H$ e $K$ ) para os aglomerados estudados.

\subsubsection{Casos que apresentam resultados catalogados}

Dos 32 casos que apresentam solução satisfatória para os parâmetros fundamentais, temos 21 que possuem solução catalogada no DAML02, portanto da nossa lista

\footnotetext{
${ }^{1}$ Tipo Espectral

${ }^{2}$ Conforme foi discutido na Seção 3.3, os valores dos parâmetros fundamentais são obtidos a partir do ajuste de isócronas à Sequência Principal do aglomerado usando o método do main sequence fitting. Valores preliminares de distância e $E(J-H)$ são usados como primeira aproximação.
} 


\begin{tabular}{|c||c|c|c||c|c|c|}
\hline \multicolumn{1}{|c||}{ Aglomerado } & \multicolumn{4}{c}{ Ajuste } & \multicolumn{3}{c|}{ DAML02 } \\
\hline \hline & distância $[\mathrm{pc}]$ & $E(J-H)$ & $\log (\mathrm{t})$ & distância $[\mathrm{pc}]$ & $E(J-H)$ & $\log (\mathrm{t})$ \\
\hline Alessi 34 & 900 & 0.04 & 8.05 & 1100 & 0.05 & 7.90 \\
\hline Alessi-Teutsch 3 & 850 & 0.07 & 8.90 & 800 & 0.03 & 8.60 \\
\hline Loden 189 & 600 & 0.02 & 8.70 & 720 & 0.04 & 8.64 \\
\hline Teutsch 35 & 720 & 0.01 & 8.25 & 700 & 0.00 & 8.37 \\
\hline
\end{tabular}

Tabela 5.1: Apresentamos 4 aglomerados cujas soluções estão em bom acordo com os valores da literatura DAML02. $\mathrm{Na}$ tabela dados referentes aos valores de distância em parcecs, excesso de cor $E(J-H)$ e $\log (\mathrm{t})$ para o ajuste e para DAML02.

original de aglomerados temos apenas 11 que são inéditos. Para os casos conhecidos (21 casos), já apresentamos uma comparação entre os valores obtidos para movimentos próprios e parâmetros fundamentais a partir deste estudo com valores do catálogo DAML02 na seção 4.1.2 do capítulo 4.

No caso dos valores dos parâmetros fundamentais obtidos, apresentamos na Tabela E em anexo: as estrelas catalogadas no SIMBAD com TE identificado na região do aglomerado bem como suas respectivas probabilidades de pertinência; os resultados de saída do programa Stax, usados como parâmetros inicias no ajuste das isócronas à sequência principal dos aglomerados no diagrama cor-magnitude, conforme foi descrito na Seção 3.3; os valores encontrados pelo ajuste e os valores catalogados no DAML02. Do conjunto de valores obtidos para distância notamos que 16 deles encontram-se na vizinhança solar, com distância igual ou menor que $1 \mathrm{Kpc}$. Estimamos valores de erros $\left(\right.$ erro $\left.=\frac{\sigma}{\sqrt{N}}\right)$ para as diferenças entre o ajuste e o valor catalogado no DAML02 para distância excesso de cor e idade respectivamente: erro $_{E(B-V)}=0.05$, erro $_{\text {dist }}=170 \mathrm{pc}$ e erro $_{\text {idade }}=150 \mathrm{Myr}$.

Citamos alguns casos onde a determinação dos parâmetros fundamentais está em bom acordo com os valores catalogados no DAML02. Apresentamos na tabela 5.1 alguns desses aglomerados, os resultados para os outros aglomerados estão na Tabela E, todos os diagramas cor-magnitude para aglomerados cujos resultados foram obtidos estão na seção 7.1 .

Os diagramas cor-magnitude dos 4 aglomerados apresentados na Tabela 5.1 
podem ser visualizados na figura 5.1.
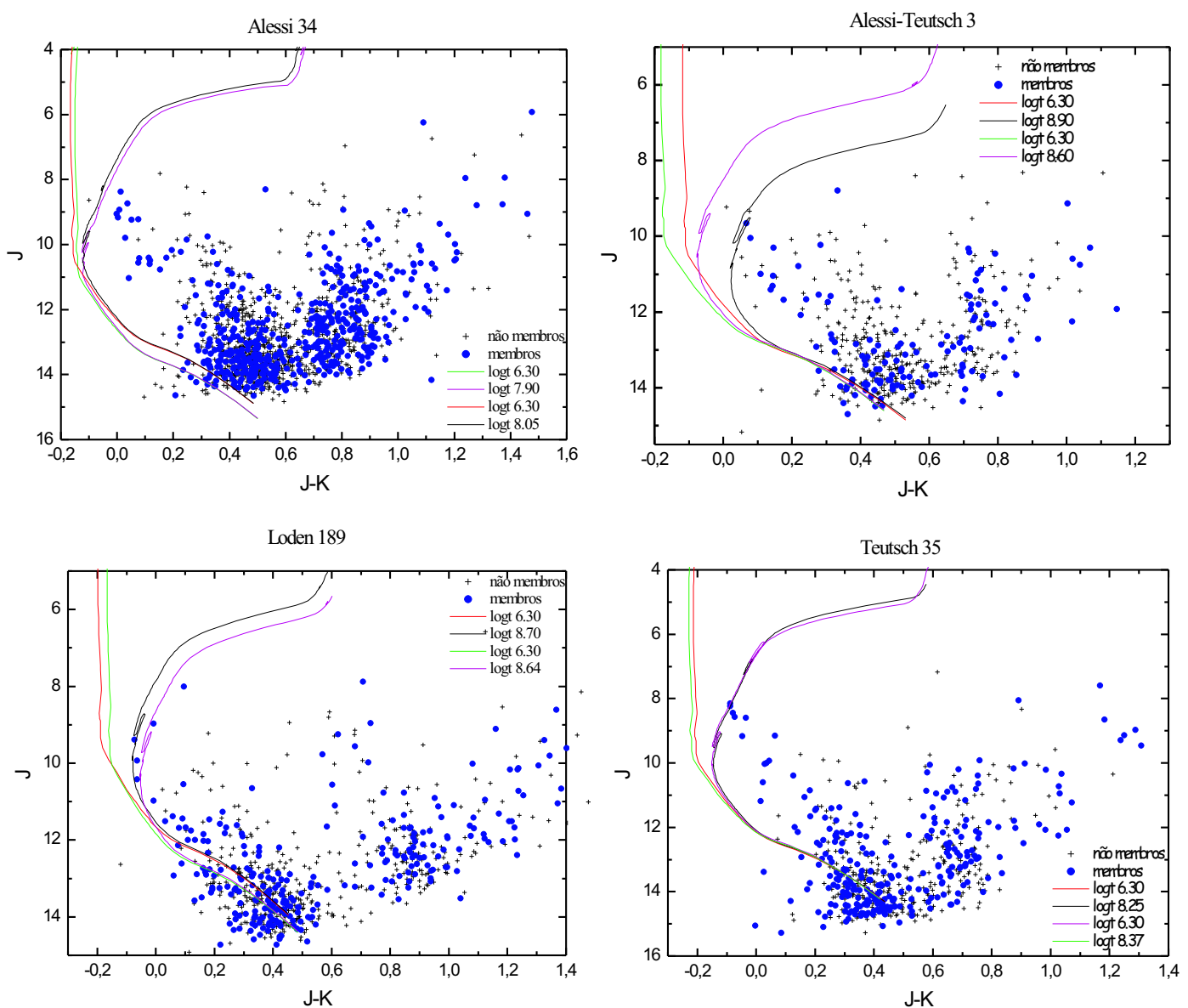

Figura 5.1: Diagramas cor-magnitude dos aglomerados Alessi 34, Alessi-Teutsch 3, Loden 189 e Teutsch 35, nas bandas $J-K \times J$ do infravemelho para os aglomerados cujas soluções de parâmetros fundamentais concordam com os resultados do DAML02. Nos diagramas dos aglomerados temos o ajuste das isócronas à sequência principal. Em vermelho e preto as isócronas cujo ajuste foi feito neste trabalho e em verde e rosa as isócronas ajustadas com os valores do DAML02. As isócronas ajustadas possuem metalicidade solar de acordo com os modelos de Padova.

Os aglomerados Loden 694, Loden 2313 e Loden 807 encontram-se com valores muito diferentes com relação aos valores catalogados em DAML02 para distâncias e excessos de cor; os valores estão na Tabela E em anexo. Os diagramas cor-magnitude para cada um desses aglomerados podem ser visualizados na figura 5.2.

A análise dos diagramas cor-magnitude na figura 5.2 possibilita ver que as isócronas que ajustam os valores do nosso ajuste se adequam melhor aos pontos na 

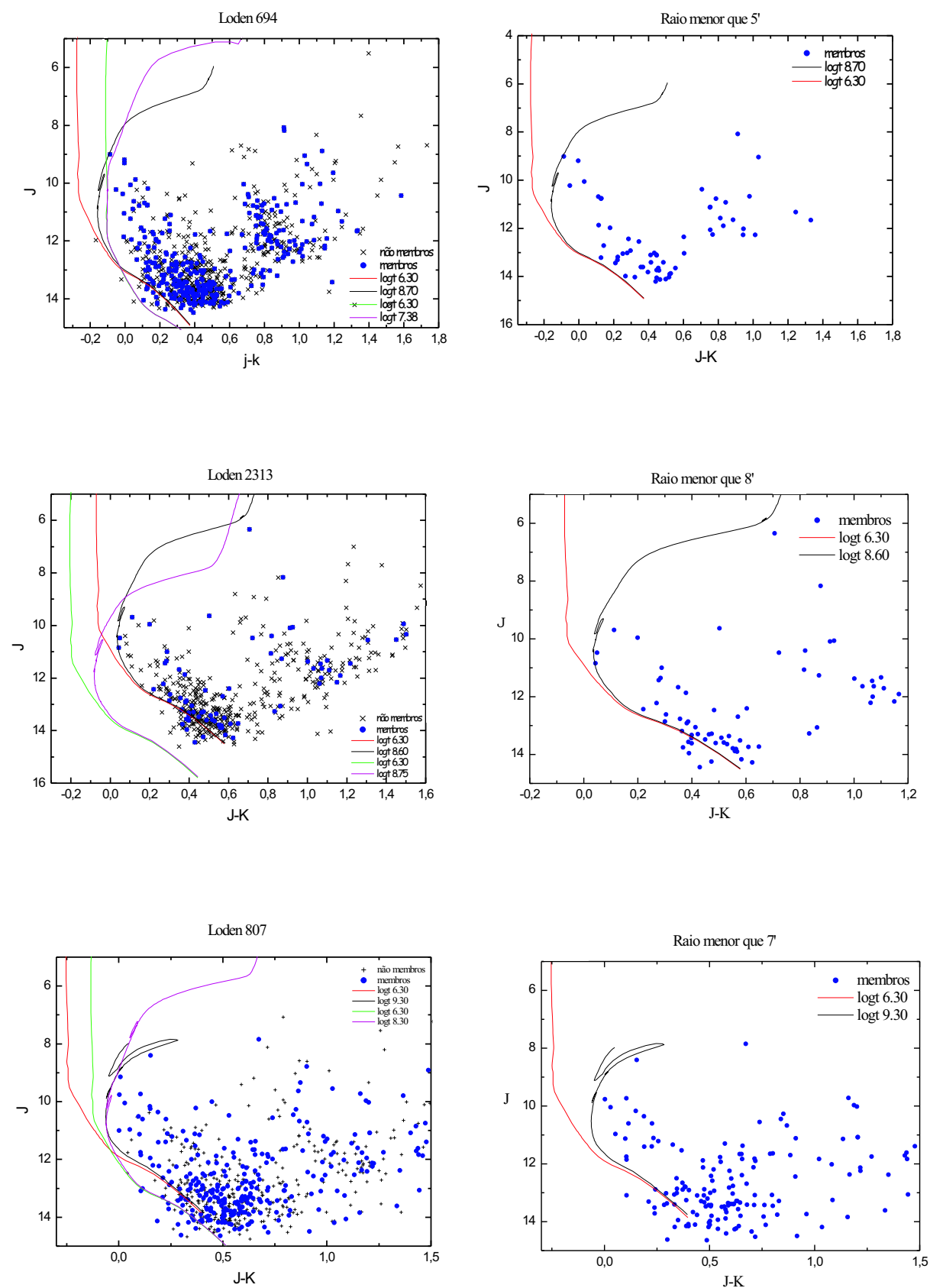

Figura 5.2: Diagramas cor-magnitude dos aglomerados Loden 694, Loden 2313 e Loden 807, nas bandas $J-K \times J$ do infravemelho para os objetos cujas soluções de parâmetros fundamentais discordam em distância e excesso de cor do DAML02. Nos diagramas dos aglomerados temos o ajuste das isócronas à sequência principal. Em vermelho e preto as isócronas cujo ajuste foi feito neste trabalho e em verde e rosa as isócronas ajustadas com os valores do DAML02. Apresentamos também os diagramas de cada aglomerado considerando apenas estrelas membros com raio menor.

sequência principal dos aglomerados. Podemos verificar esse fato também no diagrama ao lado de cada aglomerado, onde fazemos uma análise selecionando as estrelas membros que possuem um raio menor, ou seja estão mais próximas ao centro do aglomerado. Essas estrelas são provavelmente as estrelas que realmente constituem a sequência prin- 
cipal do aglomerado, tentamos ajustar as isócronas de acordo com esse diagrama e com as probabilidades de pertinência das estrelas do turn-off, também consideramos estrelas que possuem TE identificado no SIMBAD. Consideramos que a nossa determinação de parâmetros fundamentais para esses aglomerados possui valores melhores.

\subsubsection{Soluções Inéditas}

São 11 as soluções de parâmetros fundamentais dos aglomerados nunca antes estudados. Faremos aqui uma breve discussão para algumas dessas soluções, mostrando os casos bem sucedidos, todas as soluções são apresentadas na Tabela F em anexo. Empregamos no cálculo dos parâmetros o método descrito na Seção 3.3.

Os diagramas cor-magnitude dos aglomerados Harvard 16, Skiff J0619+18.5, Collinder 416, Alessi 62 e Collinder 62 estão nas figuras 5.3 e 5.4 . 

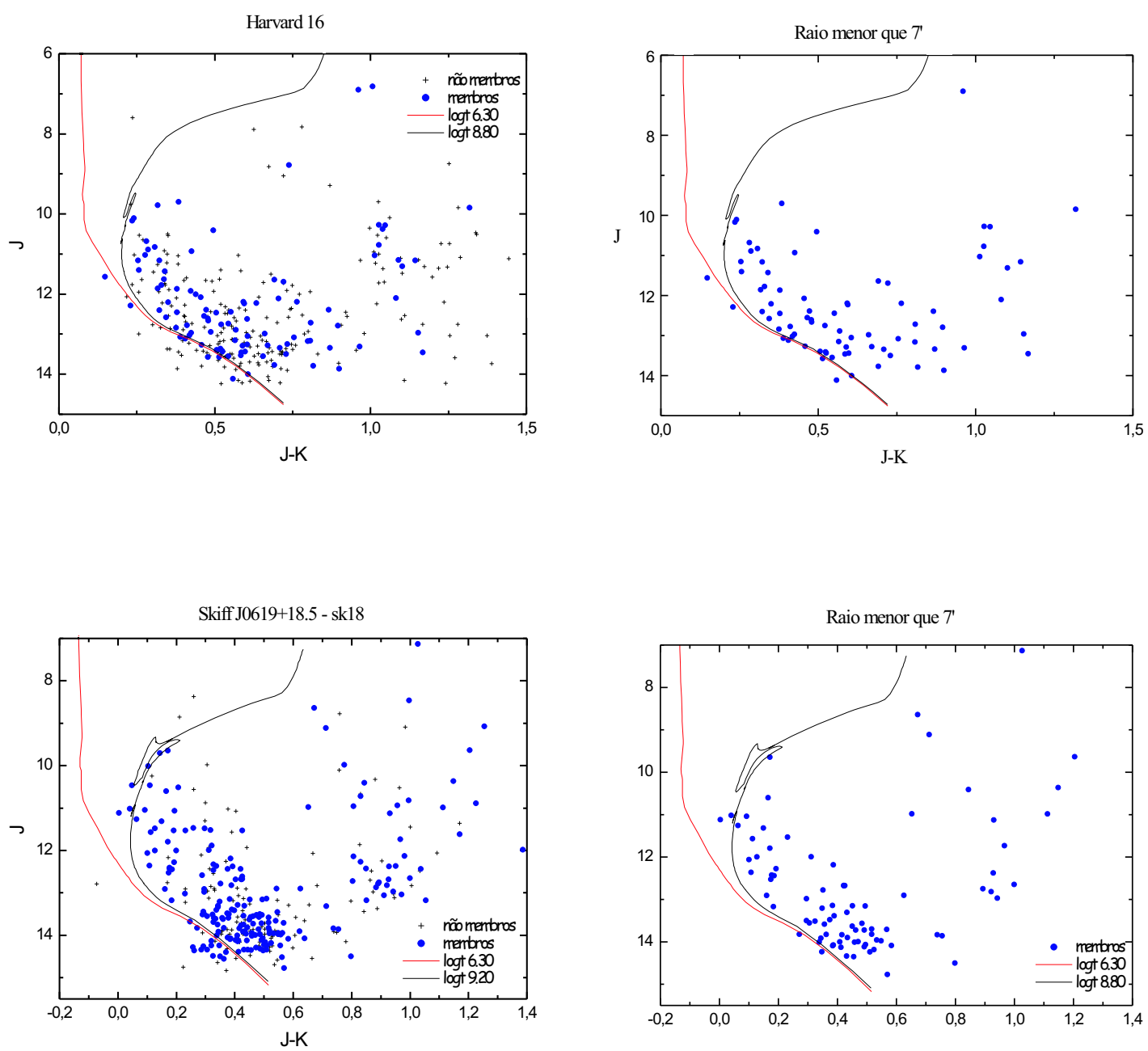

Figura 5.3: Diagramas cor-magnitude dos aglomerados inéditos Harvard 16 e Skiff J0619+18.5, nas bandas $J-K \times J$ do infravemelho. Nos diagramas dos aglomerados temos o ajuste das isócronas à sequência principal. Em vermelho e preto as isócronas cujo ajuste foi feito neste trabalho. Apresentamos também os diagramas de cada aglomerado considerando apenas estrelas membros com raio menor. Todas as isócronas ajustadas possuem metalicidade solar de acordo com os modelos de evolução de Padova.

Os vetores movimento próprio de todos os aglomerados citados possuem mesma direção como se pode perceber pelo diagrama VPD na figura 5.5. Esse é um bom indício para a determinação bem sucedida de idades e distâncias. O VPD do aglomerado Skiff J0619+18.5 pode ser visto na figura 2.1 do Capítulo 2 .

Como se pode perceber pelo ajuste no CMD, as isócronas se ajustam bem aos pontos (estrelas membro), cobrindo turn-off's e gigantes como é o caso dos aglomerados Skiff J0619+18.5, Harvard 16 e Alessi 62 . 

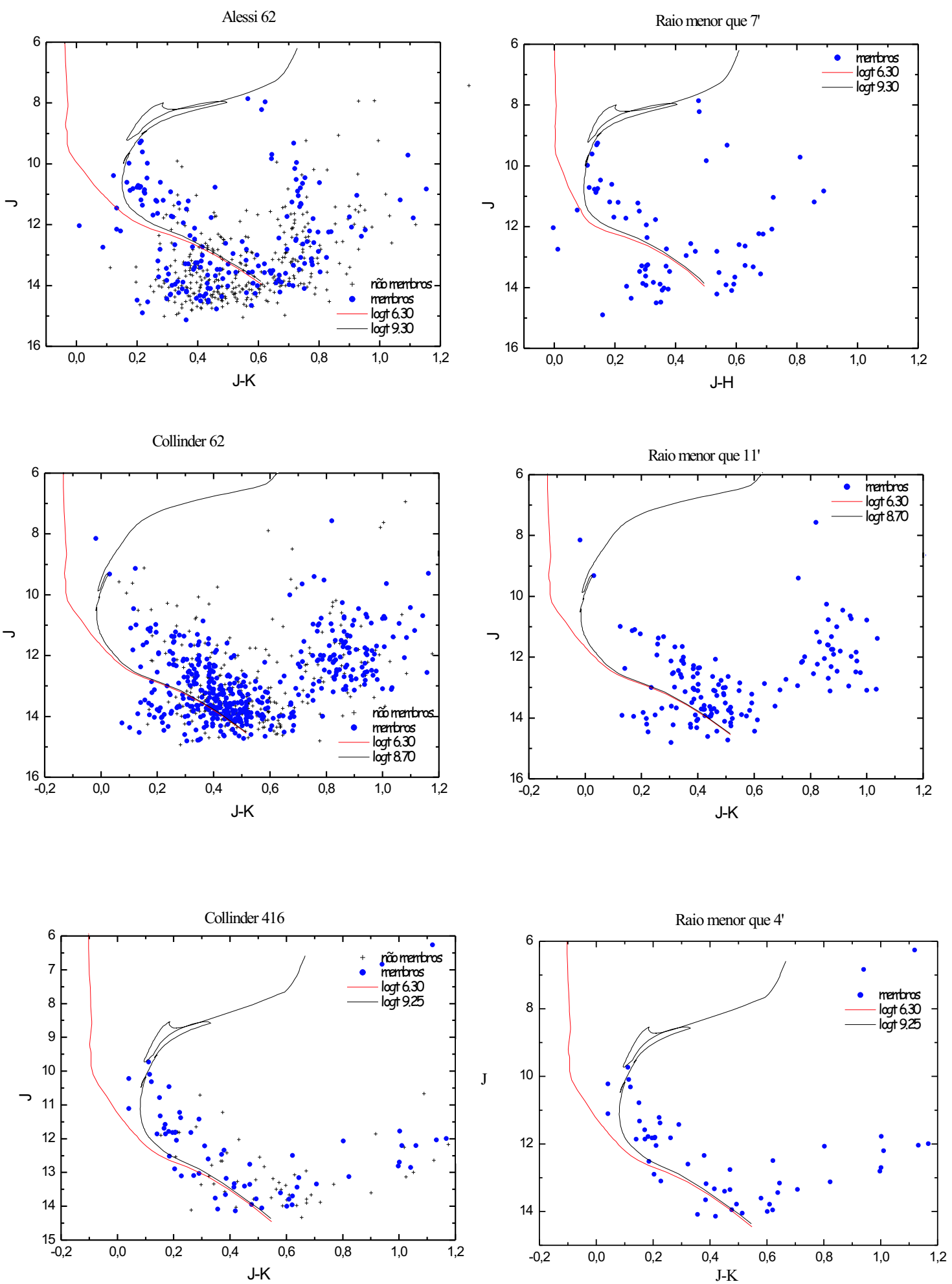

Figura 5.4: Diagramas CMD dos aglomerados Alessi 62, Collinder 62 e Collinder 416, nas bandas $J-K \times J$ do infravemelho para os objetos cujas soluções de parâmetros fundamentais são inéditas. Nos diagramas dos aglomerados temos o ajuste das isócronas à sequência principal. Em vermelho e preto as isócronas cujo ajuste foi feito neste trabalho. Apresentamos também os diagramas de cada aglomerado considerando apenas estrelas membros com raio menor. Todas as isócronas ajustadas possuem metalicidade solar de acordo com os modelos de evolução de Padova. 

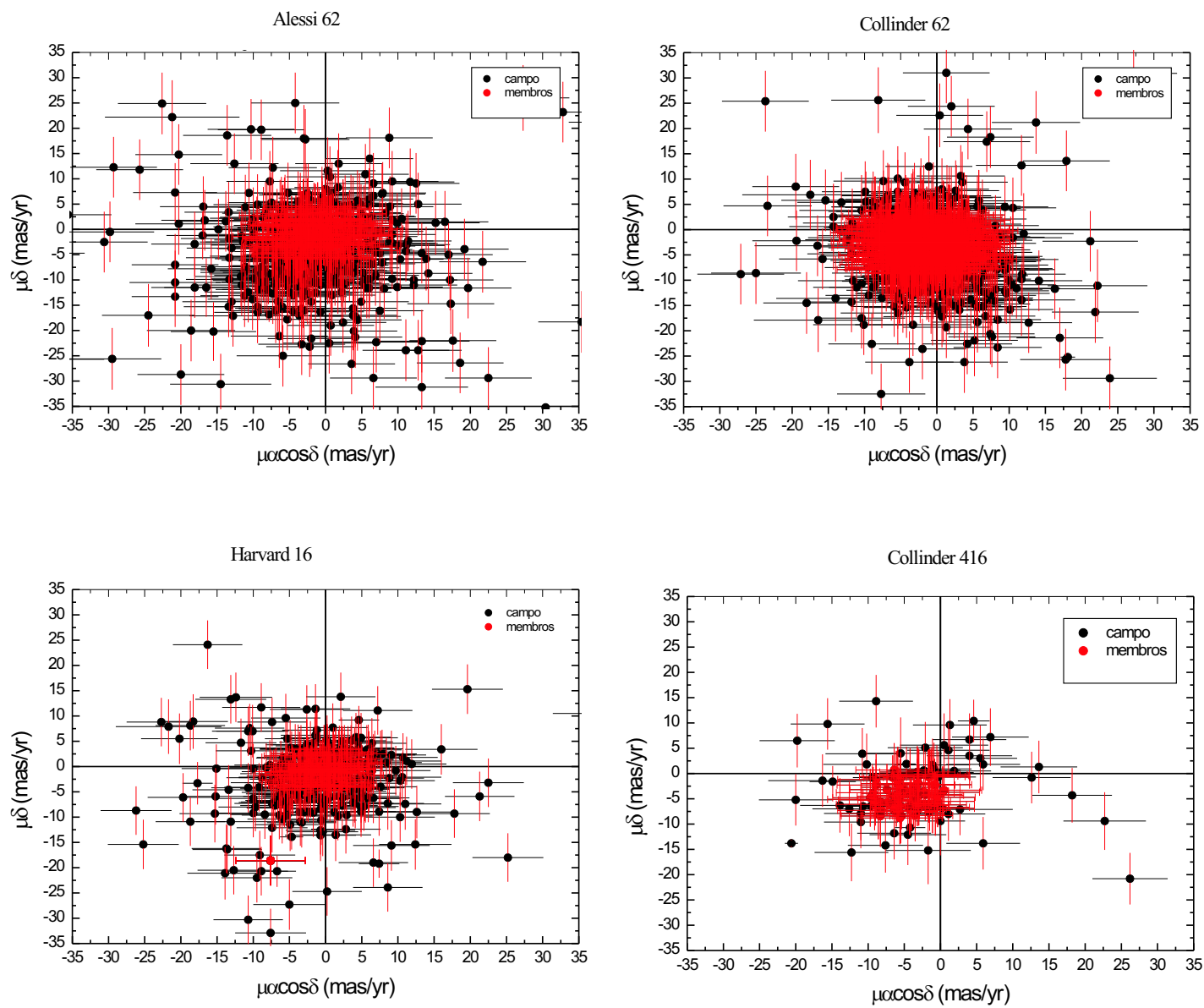

Figura 5.5: Diagramas CMD dos aglomerados, nas bandas $J-K \times J$ do infravemelho para os objetos cujas soluções de parâmetros fundamentais discordam em distância e excesso de cor do DAML02. Nos diagramas dos aglomerados temos o ajuste das isócronas à sequência principal. Em vermelho e preto as isócronas cujo ajuste foi feito neste trabalho. Apresentamos também os diagramas de cada aglomerado considerando apenas estrelas membros com raio menor. Todas as isócronas ajustadas possuem metalicidade solar (z0).

Todos os 11 aglomerados analisados apresentam valores de distância (vide tabela F) que os localizam na vizinhança solar (valores de distância menores ou iguais à $1 \mathrm{Kpc})$. 


\subsection{Estimativa de erros para os valores de distância, idades e excessos de cor}

Resolvemos alguns casos que possuem parâmetros fundamentais bem conhecidos na literatura a fim de estimar a diferença dos nossos valores com relação aos valores catalogados e usá-la como uma estimativa externa de erro em nossos cálculos de distâncias, excessos de cor e idades. Para tal, baseamo-nos nos resultados obtidos por E. Pauzen e M. Netopil [34] para uma dada amostra de aglomerados.

O trabalho citado tem como objetivo apurar a precisão dos parâmetros calculados para aglomerados abertos em trabalhos publicados e para DAML02. Ele faz uma comparação entre todos os valores dos parâmetros publicados na literatura para 72 aglomerados, sendo que para cada caso é feito um tratamento estatístico, calculando valores médios e desvios para todos os resultados publicados na literatura.

Ao todo obtivemos soluções para 20 casos da lista original de aglomerados listados no artigo de Pauzen e Netopil. Para 11 casos notamos problemas com a declinação na região dos aglomerados $\left(\delta>52^{\circ}\right)$. Para 26 casos não encontramos estrelas membros ou com TE identificado no programa SIMBAD e outros 14 casos apresentavam ouros problemas como raios muito grandes ou muito pequenos.

Para os 20 aglomerados, calculamos os movimentos próprios médios e desvios padrão em cada coordenada de acordo com o método de Zhao e He (1990)[8] descrito em detalhes na seção 2.2. Movimentos próprios na região dos aglomerados e dados fotométricos foram extraídos do catálogo UCAC2 e 2MASS respectivamente. Como o artigo de Pauzen [34] não faz o estudo para valores cinemáticos dos aglomerados, comparamos nossos valores de movimentos próprios com os catalogados no DAML02. As comparações podem ser visualizadas na figura 5.6 que mostra as diferenças entre literatura e nossos reultados nas coordenadas $\mu \alpha \cos \delta$ e $\mu \delta$ simultaneamente. 


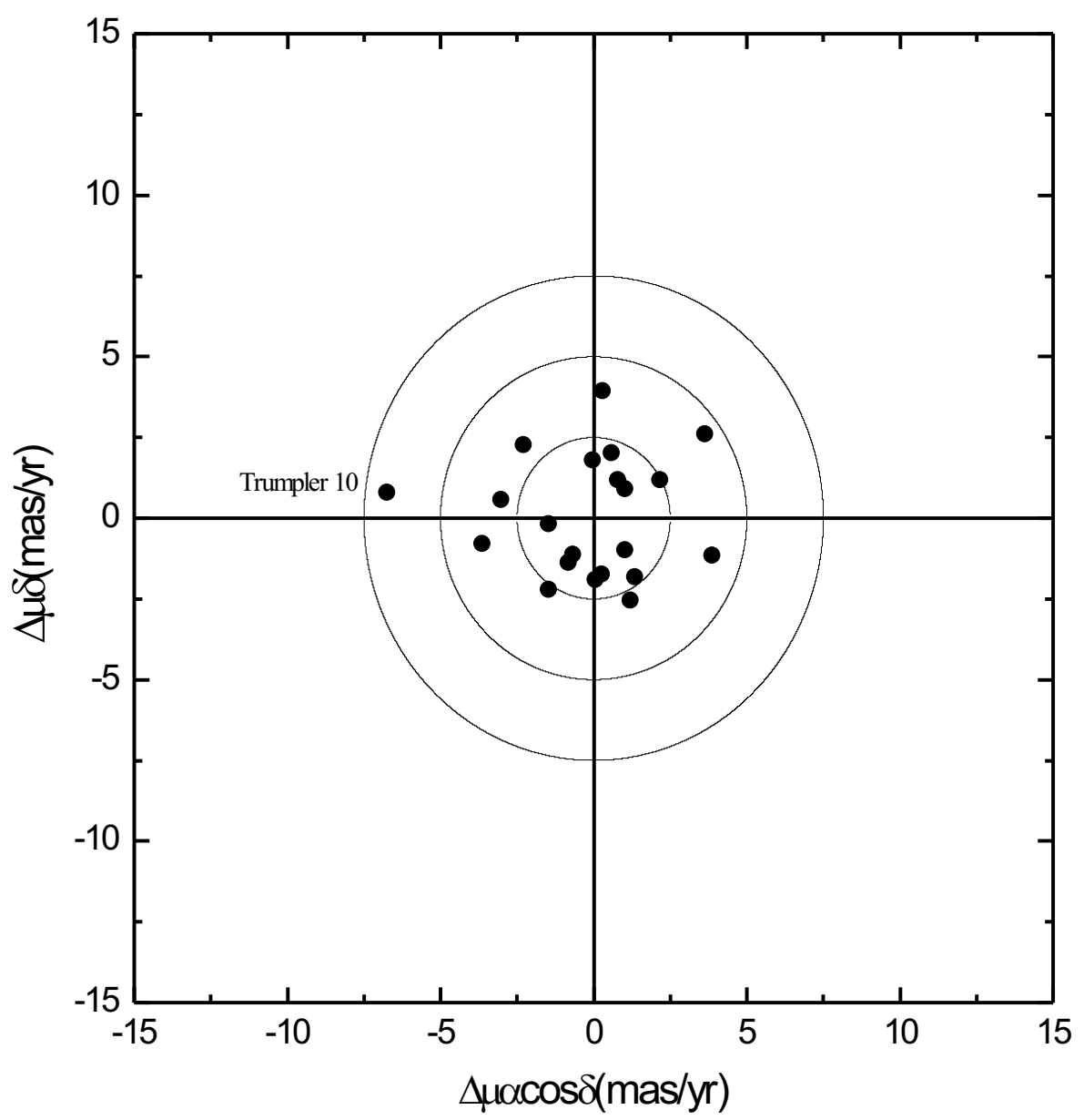

Figura 5.6: O gráfico apresenta diferenças em movimento próprio médio nas coordenadas $\mu \alpha \cos \delta$ e $\mu \delta$. Os círculos referem-se as regiões 1,2 e 3 vezes o valor de $\sigma$. Nenhum aglomerado ficou fora da região de $3 \sigma$.

De acordo com o gráfico da figura 5.6, cerca de $94 \%$ dos aglomerados estudados em comum com a amostra de Pauzen e Netopil encontram-se com movimentos próprios médios dentro da margem de $1 \sigma$; nenhum aglomerado encontra-se fora da margem de $3 \sigma$. Em todos os casos a determinação de movimentos próprios mostrou-se em boa concordância com os valores catalogados no DAML02, sendo que a média das diferenças na coordenada $\mu \alpha \cos \delta$ é -0,27 mas/yr com desvio de 2,46 e a média das 


\begin{tabular}{|c||c|c|c|}
\hline Parâmetro & Valor Médio & Desvio Padrão $(\sigma)$ & Erro $=\frac{\sigma}{\sqrt{N}}$ \\
\hline \hline distância $(\mathrm{pc})$ & 230 & 240 & 54 \\
\hline Excesso de Cor E(B-V) & $-0,05$ & 0,21 & 0,05 \\
\hline Idade (Myr) & -105 & 1160 & 260 \\
\hline
\end{tabular}

Tabela 5.2: Tabela dos valores calculados para média, desvio padrão e erros da diferença entre nossos resultados de parâmetros fundamentais e os obtidos por Pauzen para uma amostra de 20 aglomerados abertos.

diferenças na coordenada $\mu \delta$ é $0,21 \mathrm{mas} / \mathrm{yr}$ com desvio padrão de 1,77 . Os resultados indicam que temos bom material para a contrução dos diagramas cor-magnitude e determinação de distâncias, idades e excessos de cor.

Terminada a análise de movimentos próprios passamos para os resultados obtidos com os ajustes das isócronas de Padova aos diagramas cor-magnitude para obtenção de distâncias, idades e excessos de cor. Os resultados encontrados no ajuste dos parâmetros fundamentais e os calculados por Pauzen e Netopil [34], bem como os diagramas cor-magnitude podem ser visualizados na Tabela $\mathrm{C}$ e seção 7.1 .2 ambos em anexo.

A figura 5.7 traz os gráficos da comparação dos resultados para os parâmetros fundamentais. Admitimos que os resultados calculados por Pauzen e Netopil são corretos, já que foram calculados a partir de diversos valores da literatura. Dessa forma, consideramos como uma boa amostra para se inferir nossos possíveis erros no cálculo de parâmetros fundamentais. Comparando as duas amostras encontramos a média e desvio padrão das diferenças entre nossos valores e os de Pauzen e Netopilque são fornecidas na Tabela 5.2.

A partir dessa análise consideramos que às nossas determinações dos parâmetros fundamentais está associado um erro típico que assume os valores exibidos na tabela 5.2 para cada um dos parâmetros.

Usando os erros extraídos do catálogo 2MASS para cada valor de J, H e K nas bandas do infravermelho, construímos diagramas cor-magnitude para os aglomerados com os valores mais discrepantes de diferenças (diferenças em distância, excesso 

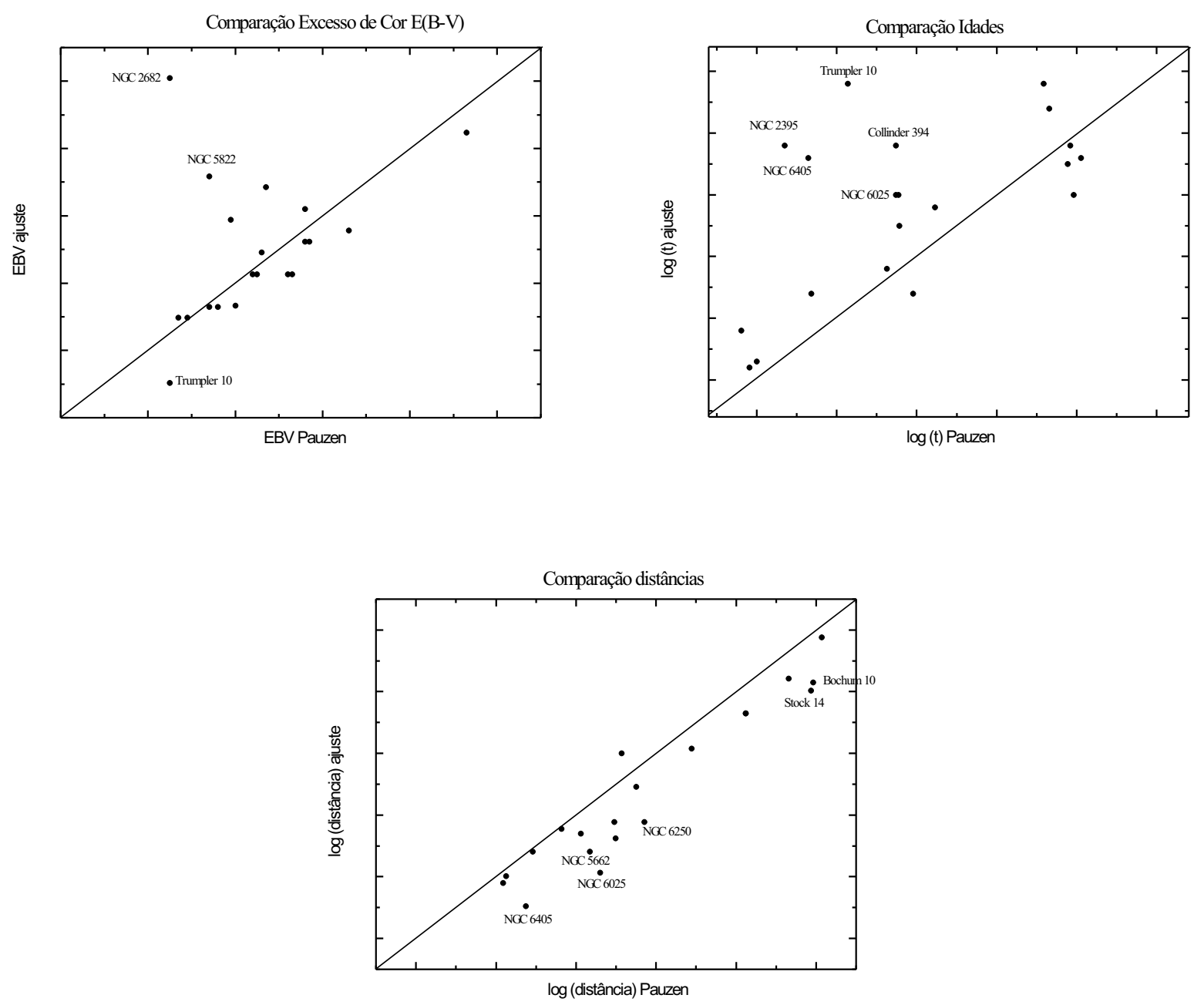

Figura 5.7: Na figura estão os gráficos comparativos entre o ajuste e os resultados obtidos por Pauzen e Netopil para 20 aglomerados abertos. Os valores são apresentados na tabela 5.2.

de cor $E(B-V)$ e idade) entre os nossos resultados e de Pauzen e Netopil. Na tabela 5.3 podemos conferir as posições de cada aglomerado extraídas do DAML02, bem como os resultados dos parâmetros fundamentais no nosso ajuste e os valores de Pauzen e Netopil.

Bochum 10 e Stock 14 apresentam maus resultados para distância, NGC 6405 e Trumpler 10 encontram-se com valores de idade discrepantes e Trumpler 10 também apresenta excesso de cor diferente do publicado por Pauzen.

As figuras 5.8 e 5.9 mostram o ajuste das isócronas aos aglomerados Bochum 10, NGC 6405, Stock 14 e Trumpler 10. Os diagrmas cor-magnitude das figuras 5.8 e 5.9 trazem os erros nas bandas $J$ e $H$ do infravermelho. 


\begin{tabular}{|c||c|c||ccc||ccc|}
\hline \multicolumn{1}{|c||}{ Nome } & \multicolumn{3}{c|}{ Posição } & \multicolumn{4}{c}{ Ajuste } & \multicolumn{3}{c}{ Pauzen } \\
\hline & $\alpha$ & $\delta$ & dist & $E(J-H)$ & logt & dist & $E(J-H)$ & logt \\
\hline \hline Bochum 10 & 104212 & -590800 & 1700 & 0.07 & 7.40 & 2472 & 0.10 & 6.90 \\
\hline NGC 6405 & 174020 & -321512 & 320 & 0.04 & 8.80 & 473 & 0.04 & 7.85 \\
\hline Stock 14 & 114348 & -623100 & 1600 & 0.07 & 7.15 & 2439 & 0.07 & 7.00 \\
\hline Trumpler 10 & 084754 & -422700 & 400 & -0.03 & 9.40 & 422 & 0.01 & 7.56 \\
\hline
\end{tabular}

Tabela 5.3: Aglomerados conhecidos utilizados no cálculo de parâmetros fundamentais: Bochum 10, NGC 6405, Stock 14 e Trumpler 10. São fornecidas posições J2000 extraídas do DAML02, sendo $\alpha$ dada em hhmmss e $\delta$ dada em $\left(o^{\prime \prime}{ }^{\prime \prime}\right)$. São dados valores do nossso ajuste para parâmetros fundamentais (distância dada em parcecs, $E(J-H)$ e $\log (\mathrm{t}))$ e também os resultados publicados por Pauzen [34] para os mesmos aglomerados.
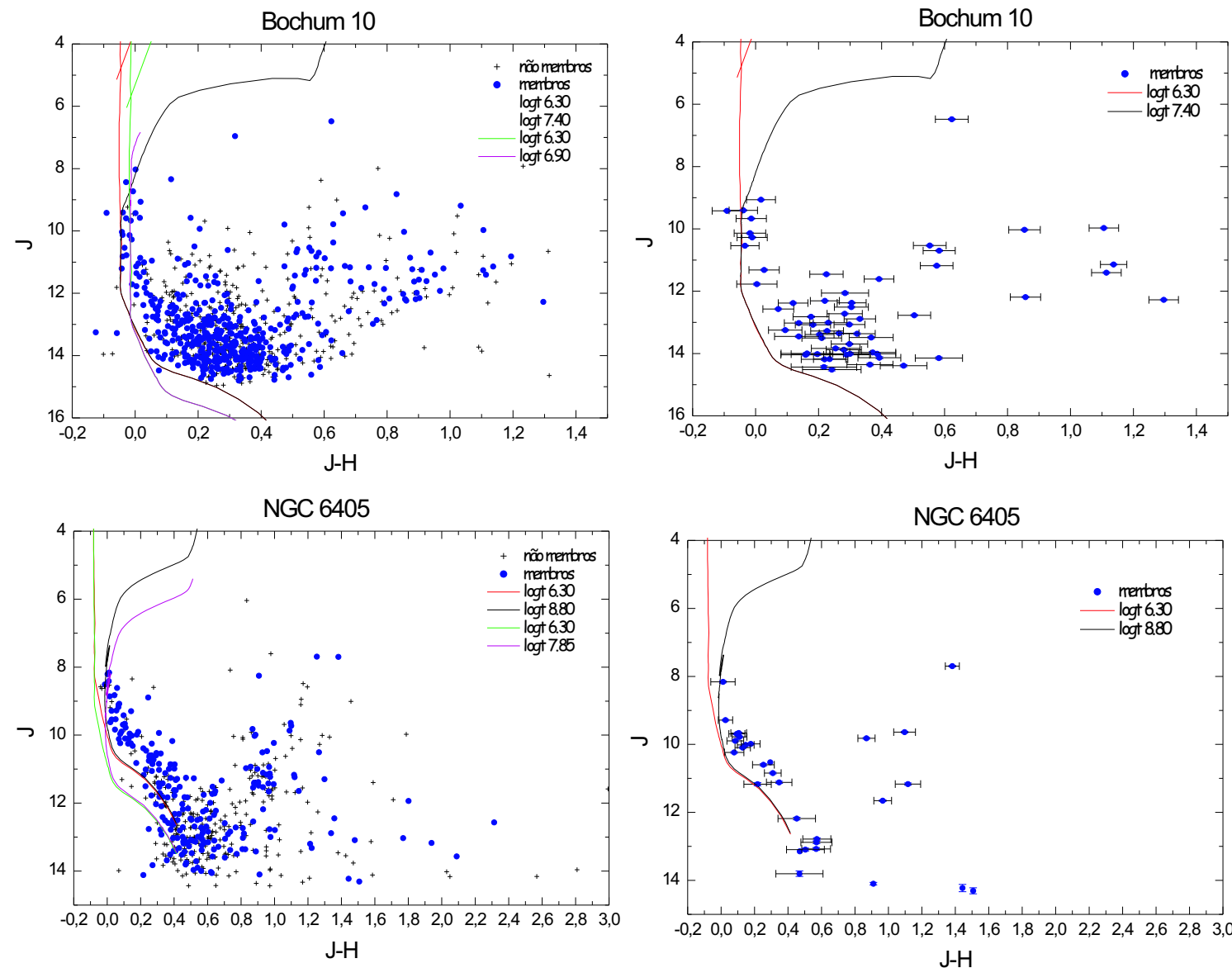

Figura 5.8: Diagramas cor-magnitude para os aglomerados Bochum 10 e NGC 6405. As isócronas foram ajustadas à sequência principal dos aglomerados no intuito de determinar distâncias, excessos de cor e idades. Em vermelho e preto as isócronas referentes ao valores do nosso ajuste e em verde e rosa referentes aos valores publicados por Pauzen e Netopil. À direita apresentamos um diagrama cor-magnitude exibindo erros nas bandas do infravermelho $J$ e $H$ extraídos do catálogo 2MASS juntamente com o ajuste dos valores de parâmetros fundamentais do nosso ajuste às isócronas (verde e rosa). Nem todas as estrelas do aglomerado apresentam erros $J$ e $H$ publicados no 2MASS. Todas as isócronas ajustadas possuem metalicidade solar de acordo com os modelos de Padova. 

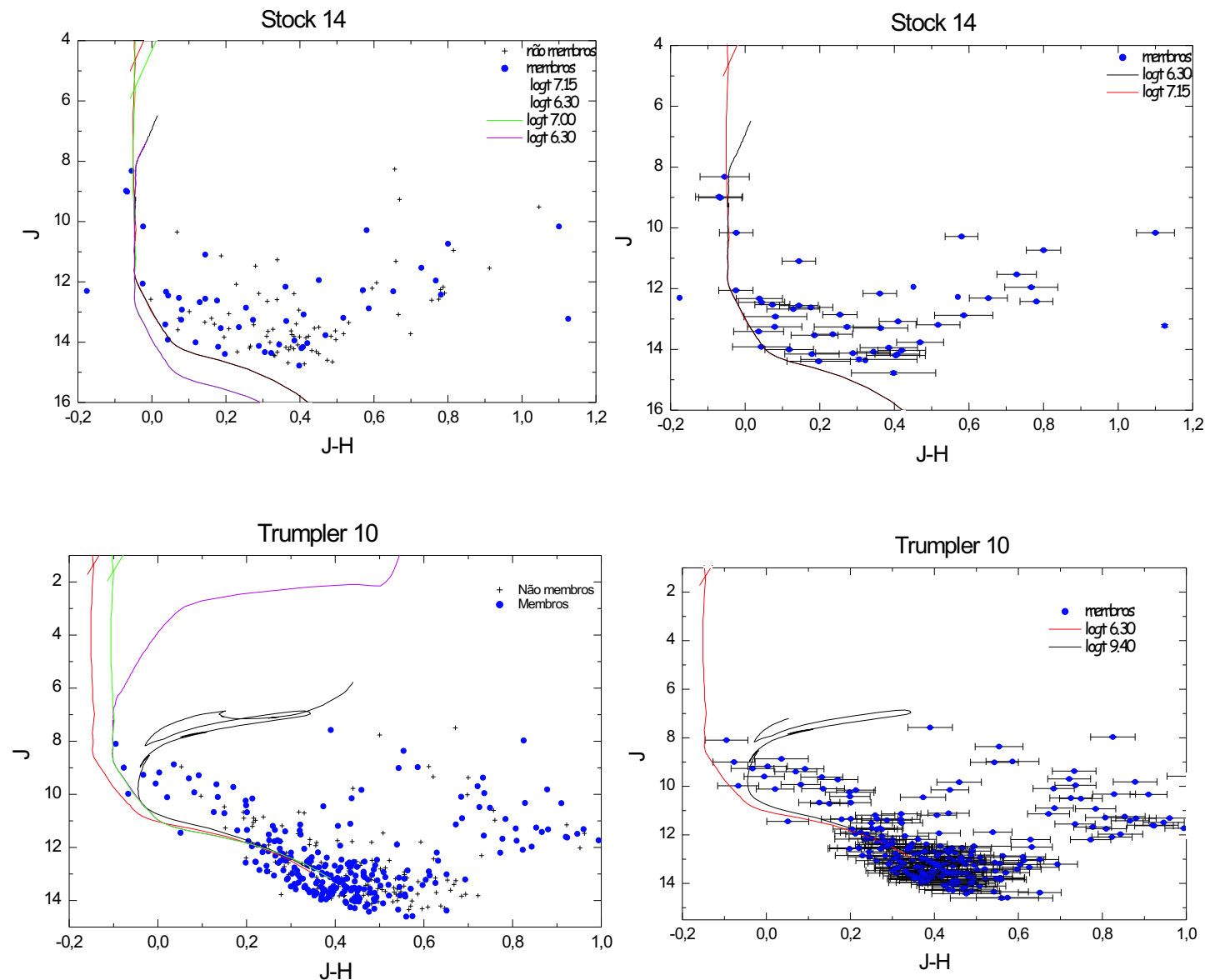

Figura 5.9: Diagramas cor-magnitude para os aglomerados Stock 14 e Trumpler 10. As isócronas foram ajustadas à sequência principal dos aglomerados no intuito de determinar distâncias, excessos de cor e idades. Em vermelho e preto as isócronas referentes ao valores do nosso ajuste e em verde e rosa referentes aos valores publicados por Pauzen e Netopil. À direita apresentamos um diagrama cor-magnitude exibindo erros nas bandas do infravermelho $J$ e $H$ extraídos do catálogo 2MASS juntamente com o ajuste dos valores de parâmetros fundamentais do nossso ajuste às isócronas (verde e rosa). Nem todas as estrelas do aglomerado apresentam erros $J$ e $H$ publicados no 2MASS. Todas as isócronas ajustadas possuem metalicidade solar de acordo com os modelos de Padova.

Um caso em que nossos resultados estão em boa concordância com os resultados de Pauzen e Netopil é o aglomerado NGC 1647. Na figura 5.10 estão os diagramas cor-magnitude do aglomerado nas bandas $J, H$ e $K$. 

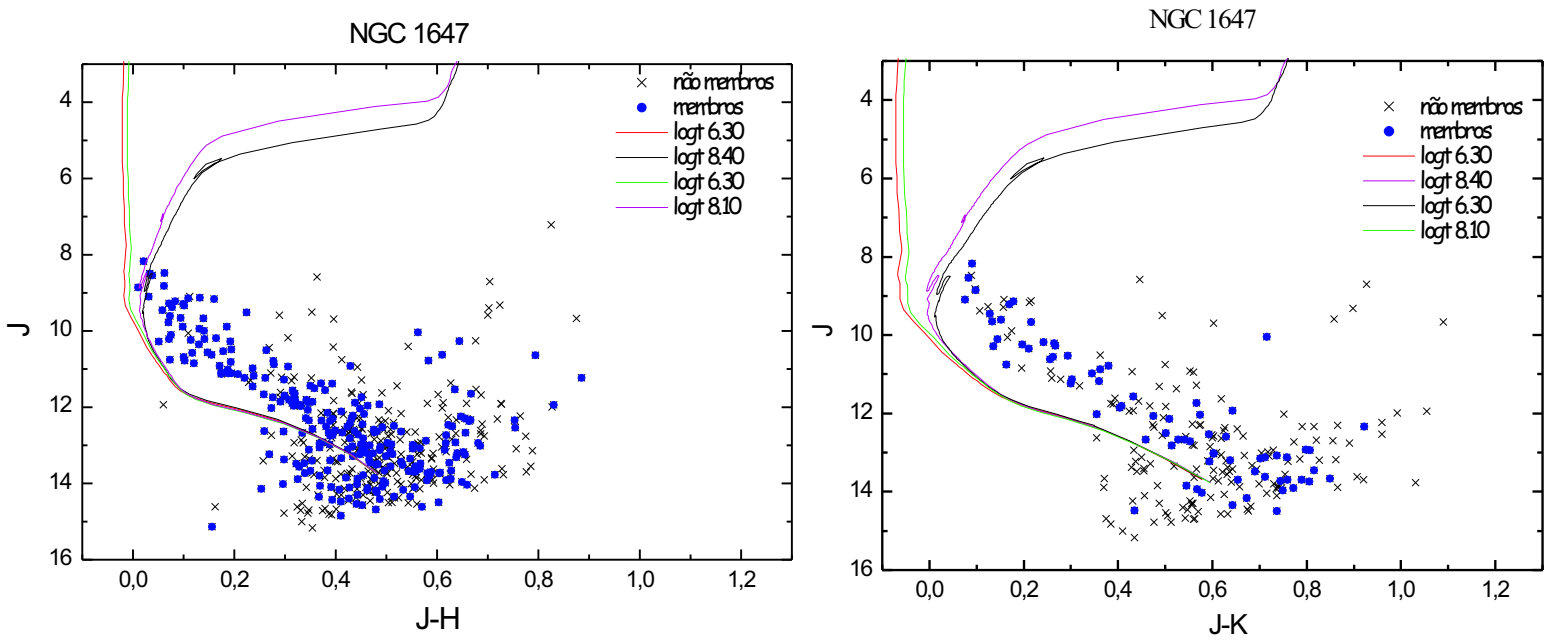

\begin{tabular}{lcccccc}
\hline Aglomerado & dist_ajuste & $E(J-H) \_a j u s t e$ & logt_ajuste & dist_Pauzen & $E(J-H) \_P a u z e n$ & logt_Pauzen \\
NGC 1647 & 480 & 0.10 & 8.40 & 492 & 0.11 & 8.11 \\
\hline
\end{tabular}

Figura 5.10: Diagramas CMD J-HxJ e J-KxJ, para o aglomerado NGC 1647. Em vermelho as estrelas com probabilidades de pertinência maiores que $50 \%$, as isócronas ajustadas com nossos valores estão em vermelho e preto, as ajustadas com os valores publicados por Pauzen e Netopil em verde e rosa. Apresentamos também os valores numéricos referentes aos ajustes dos parâmetros fundamentais. As isócronas ajustadas possuem metalicidade solar de acordo com os modelos de Padova.

A partir da figura 5.10 e da comparação dos valores dos parâmetros fundamentais, notamos que os valores dos parâmetros fundamentais para o aglomerado NGC 1647 encontram-se em boa concordância com os resultados publicados por Pauzen e Netopil.

Passamos na próxima Seção para a análise dos valores de saída do programa Stax, mencionado na Seção 3.3 do Capítulo 3. 


\subsection{Valores iniciais fornecidos para o ajuste das isó- cronas}

Conforme foi descrito desenvolvemos um programa em linguagem Java no intuito de obter valores preliminares para auxiliar o ajuste das isócronas à sequência principal dos aglomerados. Para checar o método empregado na determinação de distâncias, idades e excessos de cor, aplicamos o procedimento descrito na Seção 3.3 a uma amostra de aglomerados selecionada. Esses aglomerados foram selecionados no catálogo DAML02 de acordo com suas assinaturas no CMD, diferentes distâncias, idades e quantidades de estrelas, ou seja diferentes diâmetros aparentes.

Foi utilizada uma amostra de 11 aglomerados abertos com idades, avermelhamentos e distâncias conhecidas extraídos do DAML02. Para essa amostra de aglomerados com parâmetros bem conhecidos determinaram-se primeiramente movimentos próprios e probabilidades de pertinência, e em seguida idades, distâncias e excessos de cor. O objetivo é testar os valores iniciais calculados pelo programa Stax $^{3}$. Foram determinados movimentos próprios médios para cada um dos aglomerados em questão, bem como probabilidades de pertinência para cada estrela na região dos aglomerados conforme o método descrito na Seção 2.2. As comparações dos movimentos próprios obtidos para os 11 casos está no anexo 7 .

Usando as estrelas investigadas, especialmente aquelas que possuíam uma probabilidade de pertinência superior a 51\%, obtivemos os diagramas cor-magnitude. As soluções de idades e distâncias para esses 11 aglomerados foram obtidas a partir do método descrito na Seção 3.3. De acordo com o método main sequence fitting, isócronas de Padova foram adicionadas aos CMD e os valores iniciais para as iterações são os resultados de saída do Programa Stax. Obtiveramos soluções conforme a Tabela D.

\footnotetext{
${ }^{3} \mathrm{O}$ programa Stax foi descrito na Seção 3.3 juntamente com o método utilizado para o cálculo de distâncias e excessos de cor.
} 
Como mencionamos anteriormente a busca por tipos espectrais foi feita com auxílio do programa SIMBAD. No caso do aglomerado NGC 1907, encontramos apenas 1 estrela membro com TE no SIMBAD, com probabilidade baixa (59\%). O grau de confiabilidade do valor da determinação do programa quando se tem apenas uma estrela membro com TE identificado ou membros com probabilidade baixa é ruim, uma vez que a estrela com tipo espectral identificada pode ser considerada pelo método um membro cinemático do aglomerado mas pode não ser um membro real.

No caso do NGC 6834, temos apenas uma estrela com LC (classe de luminosidade) diferente de V. Calculamos então, apenas o excesso de cor utilizando dados do visível ${ }^{4}$.

No caso do aglomerado NGC 1750, onde temos muitas estrelas membro com TE identificado e com probabilidades muito altas $(\mathrm{P}>=89 \%)$, temos uma diferença menor que $10 \%$ entre o valor de saída do programa e o valor final do ajuste. Apresentamos gráficos comparativos para os valores de distância, excesso de cor $E(J-H)$ e $\log (\mathrm{t})$ na figura 5.11. A amostra de 11 aglomerados foi comparada com resultados catalogados no DAML02.

Notamos a partir da figura 5.11 que em geral os valores obtidos por nós encontram-se em boa concordância com os valores catalogados no DAML02. Obtivemos valores de diferenças médias de 50pc para distância e -0.02 para excesso de cor $E(J-$ $H)$. Também foram calculados os respectivos desvios padrão:

$$
\sigma_{\text {dif.E }(J-H)}=0.04 \text { e } \sigma_{\text {dif.distncia }}=190 p c .
$$

Esses valores tem como utilidade apenas fornecer uma medida do quanto nossos resultados se assemelham aos resultados do catálogo DAML02, e de acordo com eles consideramos que o nosso método é confiável para determinar distâncias, excessos de cor e idades para aglomerados abertos. Notamos ainda que os valores calculados de desvio padrão estão dentro da precisão calculada de nossas medidas, conforme expla-

\footnotetext{
${ }^{4}$ É importante lembrar aqui que ao usar LC nos referimos à classe de luminosidade da estrela e que para os cálculos de distância usamos apenas estrelas com LC V, conforme mencionamos na Seção 3.3
} 

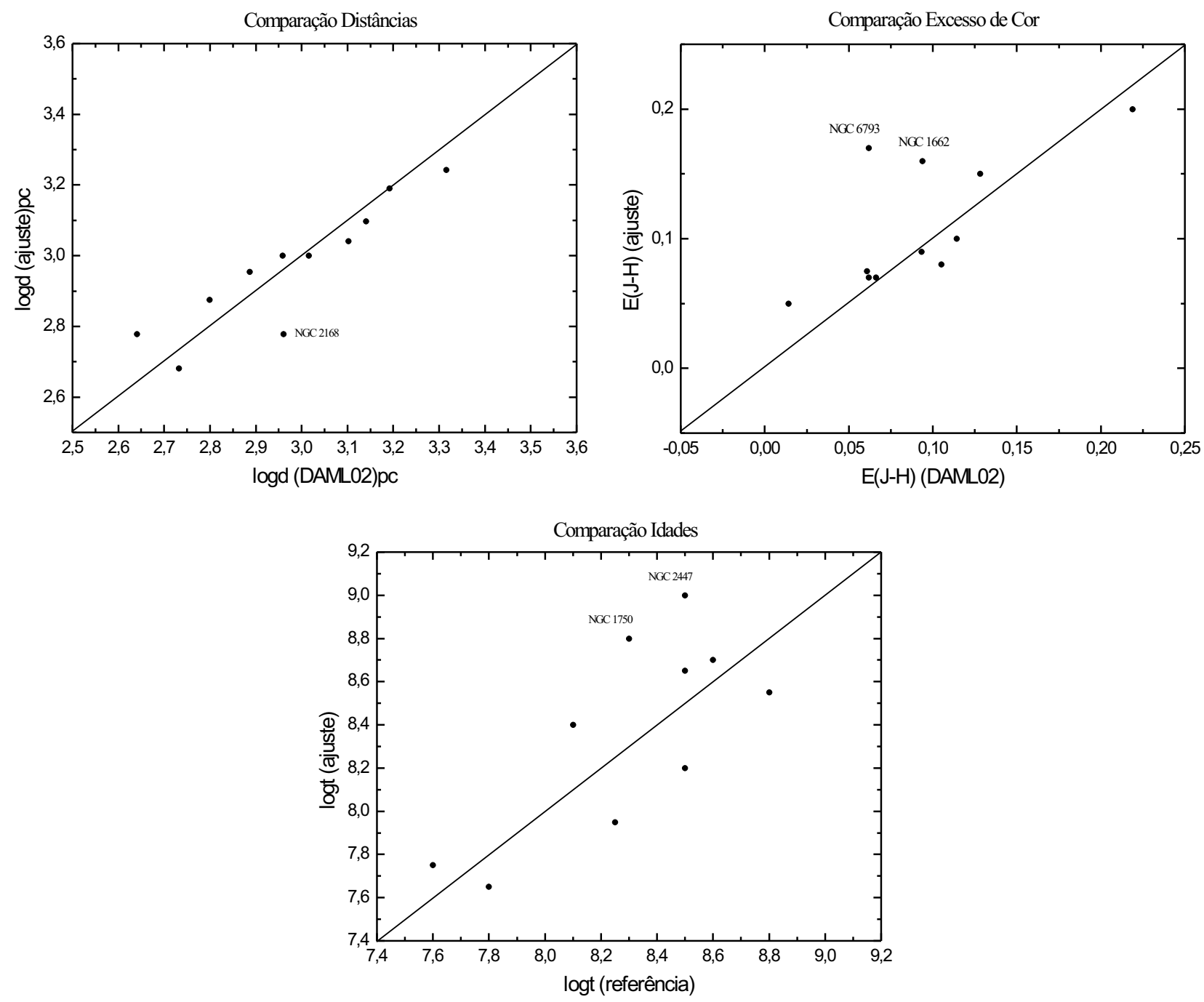

Figura 5.11: Comparação da distância, excesso de cor E(J-H) e idade dos valores obtidos com o nosso ajuste e DAML02.

namos na Seção 5.2.

Os resultados de saída do programa Stax 2 têm como finalidade apenas dar uma idéia de como ajustar as isócronas à sequência principal dos aglomerados. Nos casos onde o valor de saída do programa é muito diferente do valor do ajuste , podemos considerar que algumas das estrelas envolvidas no cálculo dos parâmetros com TE determinados podem ser membros cinemáticos do aglomerado, mas não membros de 
fato ${ }^{5}$. O fato de o aglomerado apresentar apenas uma estrela com TE identificado ou ainda se a determinação de TE for problemática no SIMBAD também compromete a análise feita pelo programa .

Na próxima Seção apresentamos os casos para os quais determinamos parâmetros fundamentais cuja solução não é inédita, ou seja os aglomerados apresentam resultados catalogados na literatura.

\subsection{Casos de difícil solução}

Não foi possível determinar a solução de parâmetros fundamentais em 34 casos. O método que descrevemos na Seção 3.3 exige que tenhamos para cada aglomerado pelo menos uma estrela com TE catalogado no SIMBAD para que seja possível o cálculo de valores iniciais de distância e excesso de cor $E(J-H)$ e além disso as estrelas não podem apresentar TE peculiar, como mencionamos na Seção 3.3.

Alguns casos oferecem maior dificuldade na determinação de parâmetros fundamentais graças a sua assinatura CMD, ou seja o diagrama cor-magniturde não apresenta uma sequência principal, turn-off ou um ramo das gigantes. Citamos os casos NGC 2364, NGC 2306, Dolidze 11 e Loden 153 como casos problemáticos para a solução graças à forma de seus diagramas cor-magnitude.

Outros aglomerados não tiveram suas soluções determinadas por não apresentarem catalogadas estrelas e TE no SIMBAD, o que impede que seja aplicado o método descrito na Seção 3.3, como já foi mencionado.

Como nenhum dos casos acima teve problemas na determinação de movimentos próprios, consultamos a identificação dos aglomerados fornecida no catálogo DAML02 e verificamos que no caso dos aglomerados cuja identificação é NGC, estão

\footnotetext{
${ }^{5}$ Isso acontece quando uma estrela de campo apresenta um movimento próprio muito com o do movimento próprio médio do aglomerado e não é membro deste. A probabilidade de pertinência dessa estrela pode ser acima de $51 \%$, o que faz dela um membro cinemático, mas na verdade trata-se de uma estrela intrusa.
} 

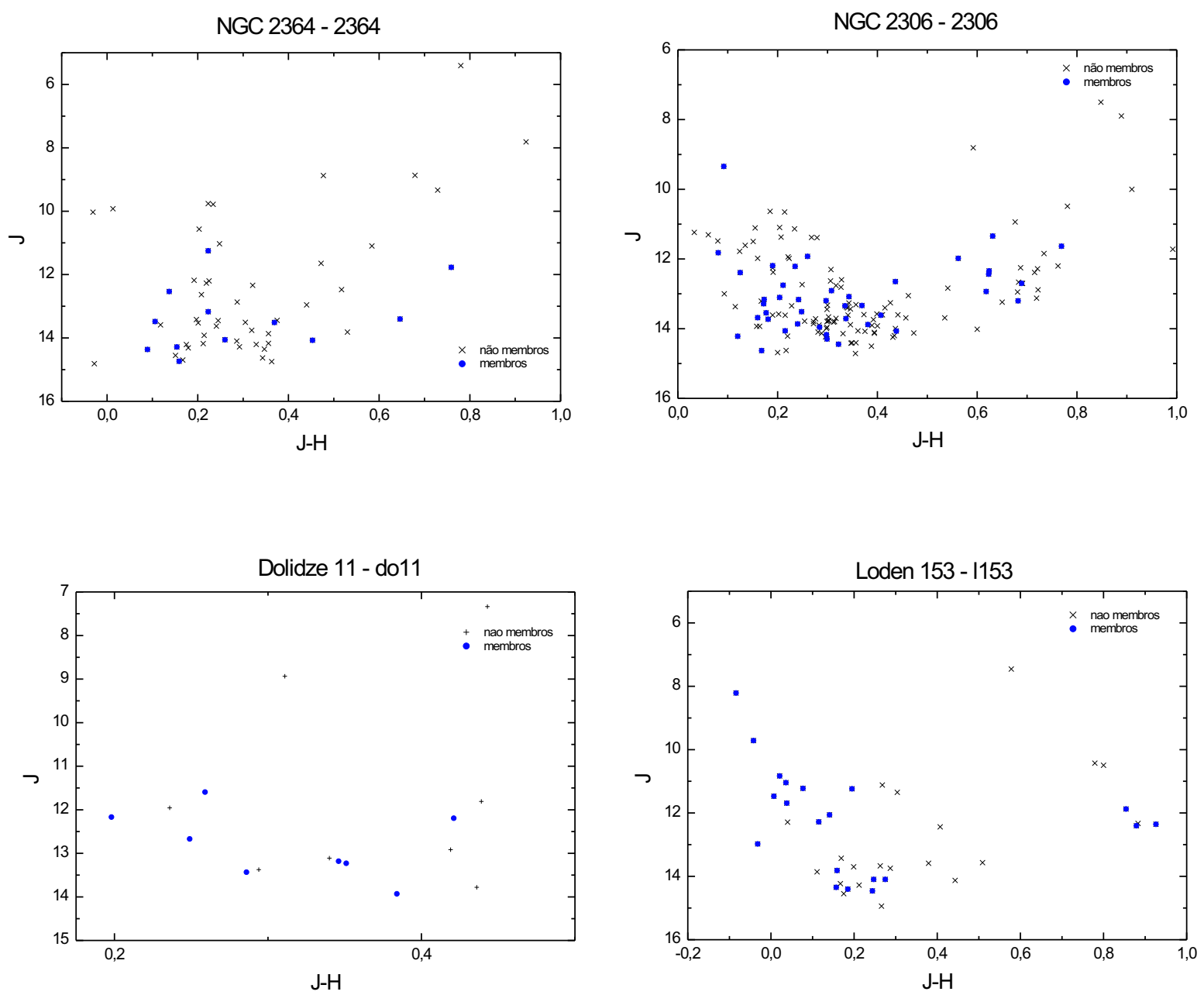

Figura 5.12: Diagramas CMD dos aglomerados NGC 2364, NGC 2306, Dolidze 11 e Loden 153, nas bandas $J-H \times J$ do infravemelho para os objetos cujas soluções de parâmetros fundamentais não foi obtida.

marcados como aglomerados não existentes. Já nos outros casos a indicação é de classificação é duvidosa. Os diagramas cor-magnitude dos aglomerados apresentam pequeno número de estrelas, o que dificulta a visualização de uma sequência principal e por cosneguinte compromete a determinação dos parâmetros fundamentais.

Propusemo-nos determinar parâmetros fundamentais para 65 casos da amostra de 319 aglomerados para os quais obtivemos soluções de parâmetros cinemáticos (moviemntos próprios). Analisamos os diagramas CMD de cada aglomerado e selecionamos 65 casos, dentre os quais obtivemos 32 soluções, sendo para 11 casos, cerca de 
17\% da amostra, tratam-se de valores inéditos. 


\section{Capítulo 6}

\section{Conclusões e Perspectivas}

Os objetivos do nosso trabalho referiram-se principalmente à determinação de parâmetros cinemáticos e fundamentais de uma amostra de aglomerados abertos nunca antes estudados na literatura, mais especificamente obtidos a partir do catálogo DAML02. Além de determinar esses parâmetros para uma série de objetos, contribuímos nesse estudo para a completeza do catálogo DAML02.

Foram investigados 850 aglomerados com $\delta<52^{0}$ e movimentos próprios médios obtidos para 319 aglomerados, utilizando dados cinemáticos obtidos do catálogo astrométrico UCAC2 e aplicando o método estatístico de Zhao e He para determinação de probabilidades de pertinência das estrelas da região estudada. Esse resultado representa um aumento de cerca de $20 \%$ na amostra de aglomerados abertos com movimentos próprios determinados no catálogo DAML02. A segurança das novas soluções cinemáticas se encontra no fato de a comparação com resultados publicados anteriormente, inclusive pelo nosso grupo, mostrar ótima concordância e sem tendências sistemáticas, conforme descrito no Capítulo 2. Foram obtidos erros nos movimentos próprios médios menores que $1 \mathrm{mas} / \mathrm{yr}$.

Da amostra com boa separação entre membros e não membros (317 ca- 
sos) foi possível determinar de forma inédita os parâmetros fundamentais (Excesso de cor, distância e idade) de 11 aglomerados. Esse número representa uma contribuição de $4 \%$ do total de aglomerados com parâmetros fundamentais conhecidos no catálogo DAML02. Além desses casos determinamos os parâmetros fundamentais de mais 21 casos, sendo que esses já tinham resultados apresentados na literatura. Dos 11 casos inéditos concluímos que todos encontram-se a uma distância menor ou igual a 1 kpc, ou seja, localizam-se na vizinhança solar.

Através de uma comparação com uma amostra de aglomerados detalhadamente investigada por Paunzen e Netopil [34], foi possível estimar (de forma externa) os erros de nossos resultados de parâmetros fundamentais. Basicamente chegamos à conclusão de que os resultados aqui apresentados têm incertezas semelhantes aos resultados do catálogo DAML02, ou seja: $\operatorname{erro} E(J-H)=0,05$, errodistância $=54 \mathrm{pc}$ e erroidade $=260 \mathrm{Myr}$.

Finalmente é importante ressaltar que para a determinação de distância através do main sequence fitting, empregado nesse trabalho, é fundamental inferir previamente o excesso de cor, caso contrário múltiplas soluções são posíveis através das análises dos diagramas cor-magnitude. Para a estimativa dos excessos de cor optamos por utilizar os tipos espectrais das estrelas selecionadas como membros na análise cinemática. Os tipos espectrais foram obtidos diretamente do SIMABD de forma que descartamos estrelas com tipos espectais peculiares em nossas análises. Esse procedimento explica o pequeno número de aglomerados com soluções para os parâmetros fundamentais, pois de forma geral o número de estrelas com tipo tipo espectral determinado ainda é muito pequeno quando comparado às milhares de estrelas que investigamos nesse trabalho.

Para o futuro esse trabalho contribuiu também selecionando aglomerados candidatos à serem observados no Survey Sul, em andamento no LNA. A contrapartida de membros cinemáticos pode e deve contribuir nas análises fotométricas para 
determinação de distâncias e idades. Além disso é importante lembrar que o trabalho continuará para $>52^{\circ}$ assim que o catálogo astrométrico UCAC3 for publicado. 


\section{Capítulo 7}

\section{Apêndices}

\section{I - Resultados Stax}

Com o intuito de checar o método empregado na determinação de distâncias, idades e excessos de cor, aplicamos o procedimento mencionado na seção 2.2 a uma amostra de 11 aglomerados extraída do DAML02. Para essa amostra de aglomerados com parâmetros bem conhecidos determinamos primeiramente movimentos próprios e probabilidades de pertinência, e usando as estrelas com probabilidades de pertinência maiores que 50\% construímos os diagramas cor-magnitude a fim de conhecer valores de distâncias, excessos de cor e idades.

Nesta Seção fazemos a análise dos movimentos próprios determinados para a amostra de aglomerados mencionada. A seguir a tabela 7.1 lista os aglomerados conhecidos utilizados nos cálculos, bem como a comparação dos valores obtidos para movimentos próprios com os valores de um artigo publicado pelo grupo [4].

O gráfico da figura 7.1 apresenta a diferença nas coordenadas de movimentos próprios $\mu \alpha \cos \delta$ e $\mu \delta$ para valores obtidos em nosso ajuste e os publicados no artigo.

A comparação das duas amostras foi feita através do cálculo das diferenças 


\begin{tabular}{|c||c|c|c|c||c|c|c|c|}
\hline \multicolumn{1}{|c||}{ Aglomerado } & \multicolumn{4}{c}{ Ajuste } & \multicolumn{6}{c|}{ Artigo } \\
\hline \hline & $N_{c}$ & $\mu \alpha \cos \delta$ & $\mu \delta$ & erro & $N_{a}$ & $\mu \alpha \cos \delta$ & $\mu \delta$ & erro \\
\hline \hline 2168 & 270 & 0,76 & $-3,09$ & 0,11 & 600 & 0,51 & $-3,2$ & 0,15 \\
\hline 6793 & 30 & 2,36 & $-1,93$ & 0,57 & 60 & 2,09 & $-3,37$ & 0,51 \\
\hline 6834 & 50 & $-1,21$ & $-3,09$ & 0,44 & 70 & $-1,17$ & $-3,56$ & 0,38 \\
\hline 6940 & 300 & $-3,06$ & $-6,07$ & 0,18 & 380 & $-3,39$ & $-6,43$ & 0,17 \\
\hline 1907 & 40 & $-2,56$ & $-3,29$ & 0,55 & 26 & $-2,77$ & $-4,82$ & 0,66 \\
\hline 2099 & 250 & 2,22 & $-6,01$ & 0,17 & 250 & 2,47 & $-6,65$ & 0,17 \\
\hline $\operatorname{tr} 21$ & 55 & $-6,63$ & $-3,37$ & 0,43 & 55 & $-7,29$ & $-3,91$ & 0,45 \\
\hline 2447 & 90 & $-5,12$ & 3,62 & 0,29 & 75 & $-5,12$ & 3,66 & 0,33 \\
\hline 1750 & 111 & $-2,12$ & $-2,67$ & 0,22 & 140 & $-1,93$ & $-3,29$ & 0,23 \\
\hline 1647 & 200 & $-0,28$ & $-1,84$ & 0,17 & 230 & $-0,54$ & $-2,64$ & 0,19 \\
\hline 1662 & 70 & $-0,74$ & $-1,87$ & 0,24 & 55 & $-1,37$ & $-2,37$ & 0,28 \\
\hline
\end{tabular}

Tabela 7.1: Objetos com parâmetros conhecidos cujos movimentos próprios foram investigados. Mostramos na tabela a comparação entre os valores obtidos com o nosso ajuste e os publicados em um artigo do grupo [4] para os movimentos próprios dos aglomerados nas duas coordenadas $\mu \alpha \cos \delta$ e $\mu \delta$ em mas/yr. O erro mostrado é calculado de acordo com a relação: erro $=\frac{\sigma}{\sqrt{n_{c}-1}}, N_{c}$ e $N_{a}$ denotam o número de estrelas membro consideradas em nossa análise e no artigo respectivamente.

em cada coordenada que forneceram uma média nas diferenças igual a -0,19mas/yr para $\mu \alpha \cos \delta$ com desvio padrão de 0,28 e -0,63 mas/yr para $\mu \delta$ com desvio padrão de 0,53 . De acordo com o gráfico acima nenhum aglomerado está fora da margem de 3 vezes o desvio padrão.

O caso do aglomerado NGC 6793 é o caso que apresenta maiores discrepâncias quanto à sua solução para movimentos próprios médios. Para esse caso sugerimos que o fato de terem sido adotadas menos estrelas como membros influenciou na determinação de movimentos próprios médios nas coordenadas. Também de acordo com a Tabela percebemos que o erro encontrado nessa determinação é maior, o que faz do valor encontrado no artigo um valor melhor. Para os outros casos os erros encontrados se assemelham. 


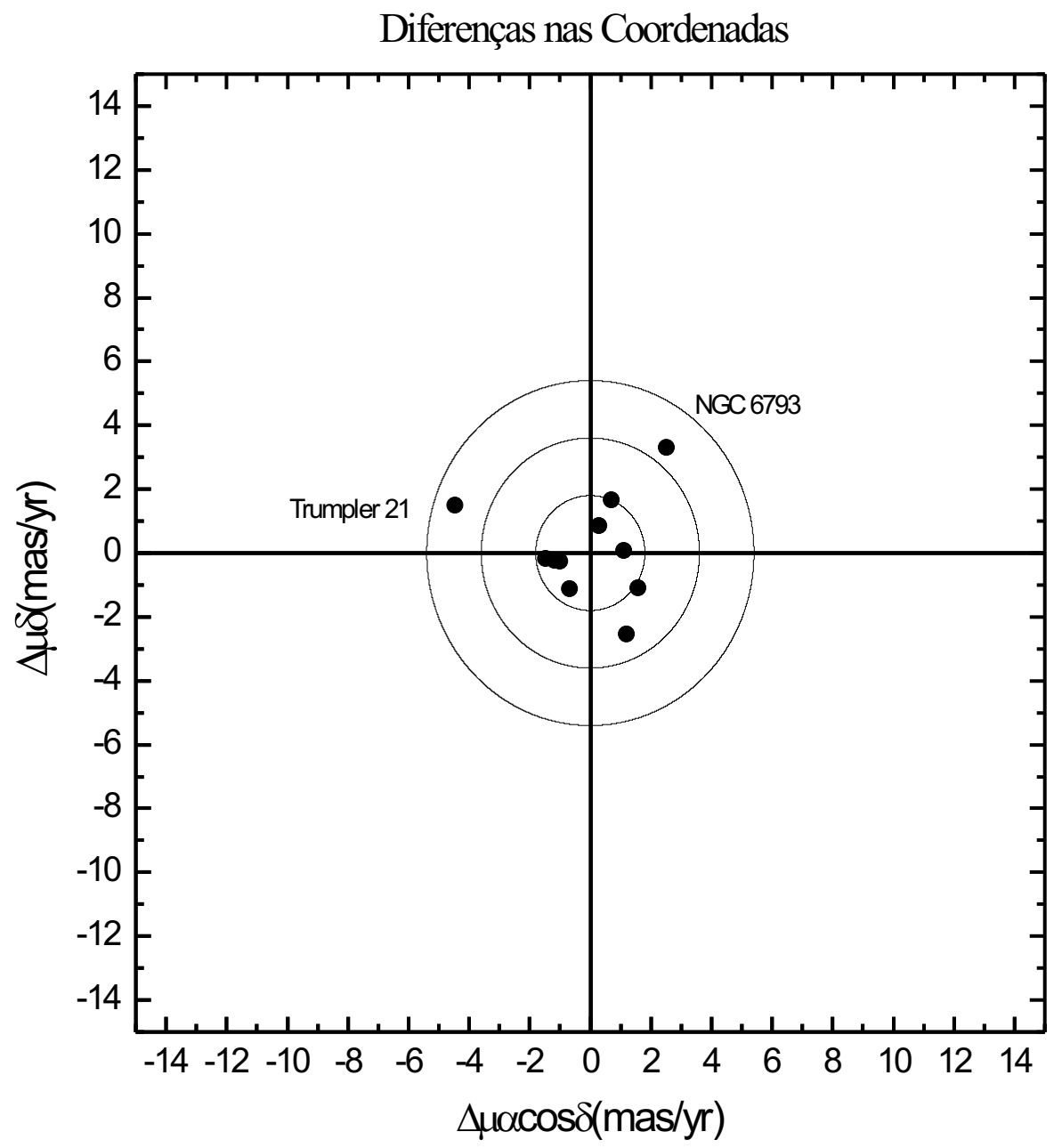

Figura 7.1: O gráfico apresenta diferenças em movimento próprio médio nas coordenadas $\mu \alpha \cos \delta$ e $\mu \delta$. Os círculos mostram as regiões de desvio padrão nas coordenadas, sendo iguais a 1,2 e 3 vezes o valor de $\sigma$.

\subsection{Diagramas CMD dos aglomerados estudados}

Apresentamos os diagramas CMD para os aglomerados abertos que tiveram seus parâmetros fundamentais investigados neste projeto. Junto aos diagramas estão as isócronas ajustadas para determinação de distâncias, excessos de cor e idades. 


\subsubsection{Casos estudados no projeto}

Nesta subseção apresentamos os diagramas CMD para os aglomerados cuja soluções foram obtidas por nós para a amostra selecionada de aglomerados na versão 2.3 do catálogo DAML02 e que tiveram seus movimentos próprios determinados a partir do método descrito na Seção 2.2. São 32 casos diagramas, os valores dos ajustes constam das Tabelas E e D.

Os aglomerados que apresentavam resultados catalogados na literatura contam com isócronas ajustadas de acordo com os valores dos parâmetros fundamentais DAML02.

\subsubsection{Casos em comum com a amostra de Pauzen e Netopil}

Apresentamos os diagrmas CMD para os 20 casos de aglomerados abertos para os quais estudamos em comum com a amostra selecionada de Pauzen e Netopil. Junto aos diagramas estão as isócronas ajustadas para determinação de distâncias, excessos de cor e idades. Os valores dos ajustes constam da Tabela $\mathrm{C}$ em anexo. 

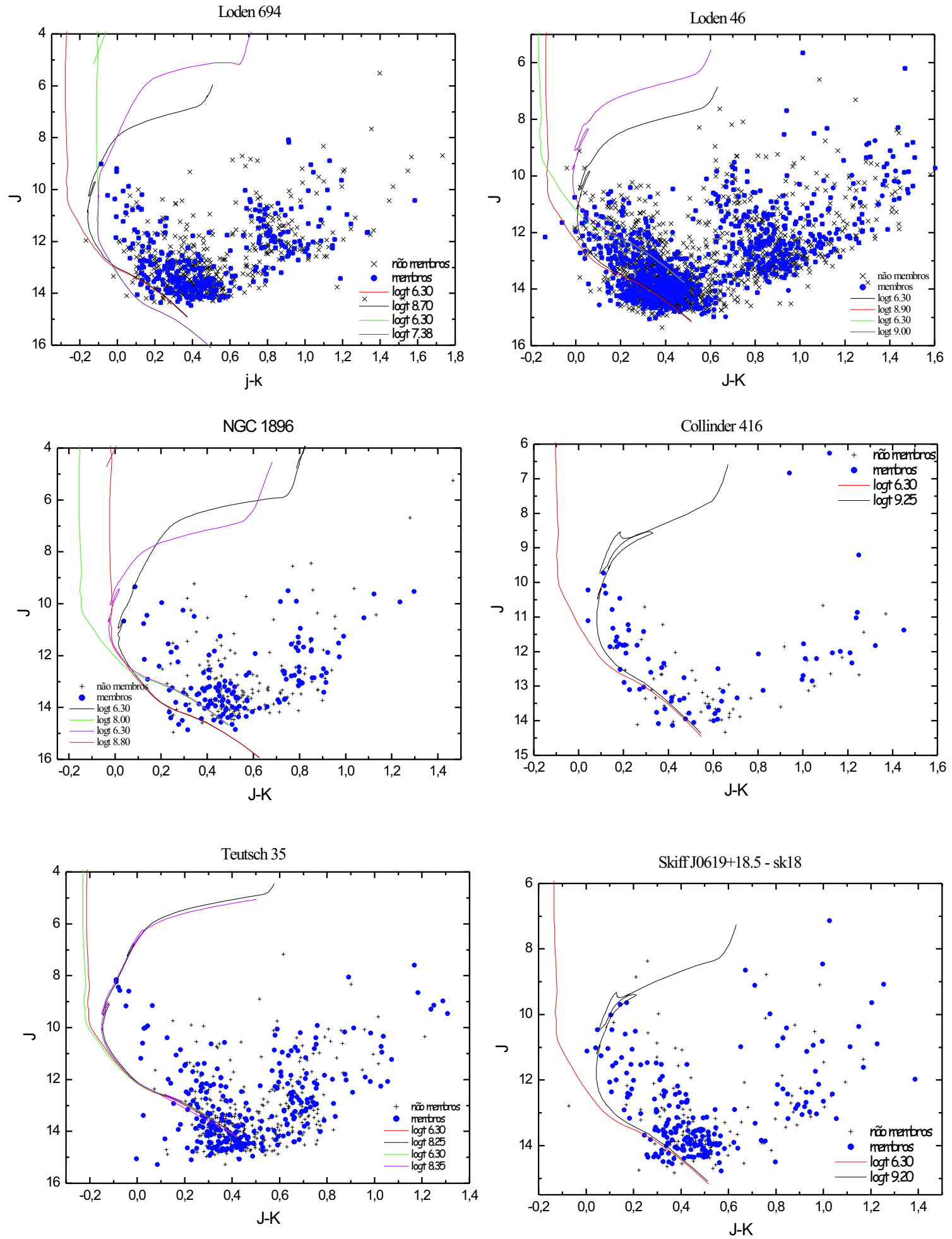

Figura 7.2: Diagramas CMD dos aglomerados Loden 694, Loden 46, NGC 1896, Collinder 416, Teutsch 35 e Skiff J0619+18.5 nas bandas $J-K \times J$ do infravermelho. Nos diagramas dos aglomerados temos o ajuste das isócronas à sequência principal. Em vermelho e preto as isócronas cujo ajuste foi feito neste trabalho e em verde e rosa as isócronas ajustadas com os valores do DAML02 para os casos que possuem valores catalogados na literatura. As isócronas ajustadas possuem metalicidade solar de acordo com os modelos de Padova. 


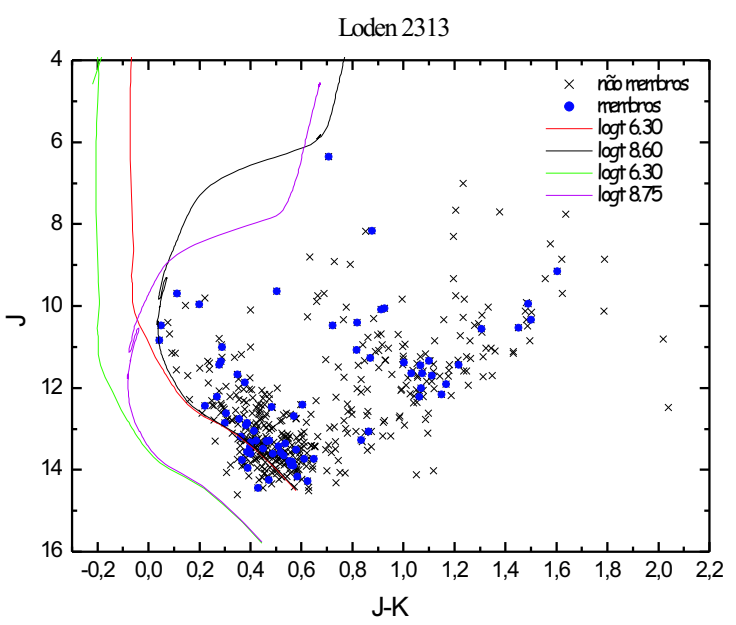

Loden 306

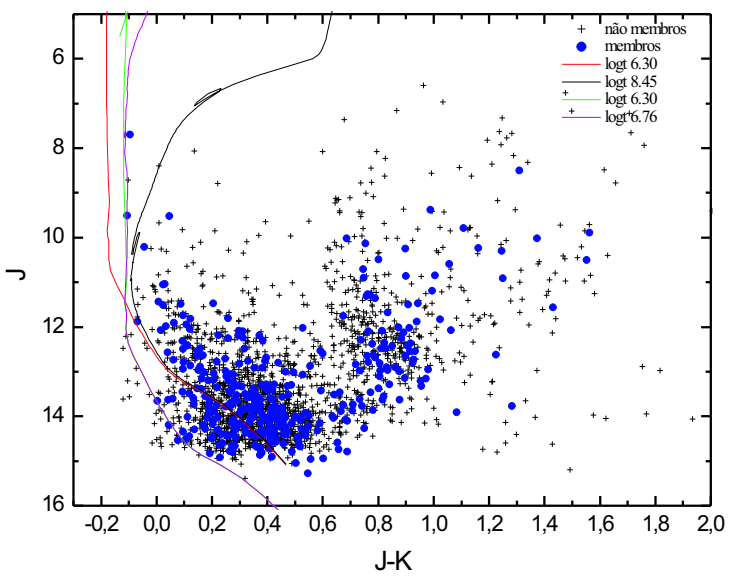

Loden 189
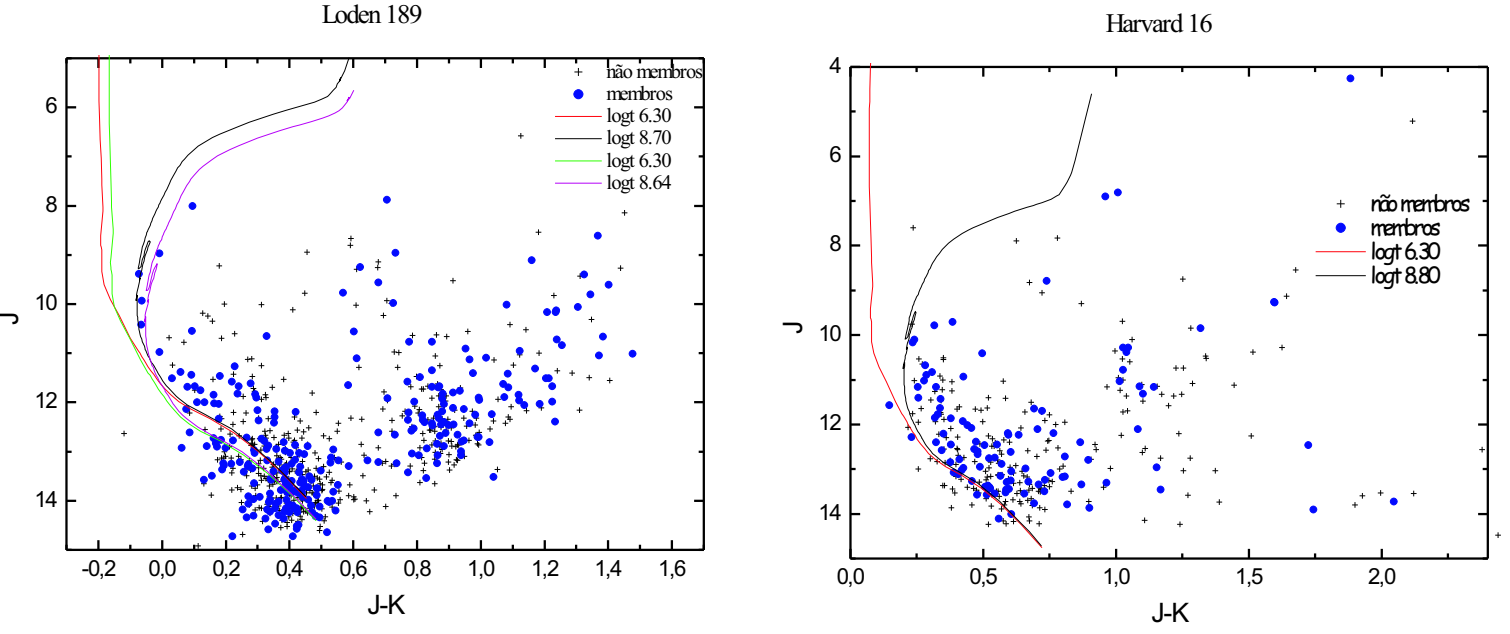

Eso $332-08$ - e332

Collinder 62
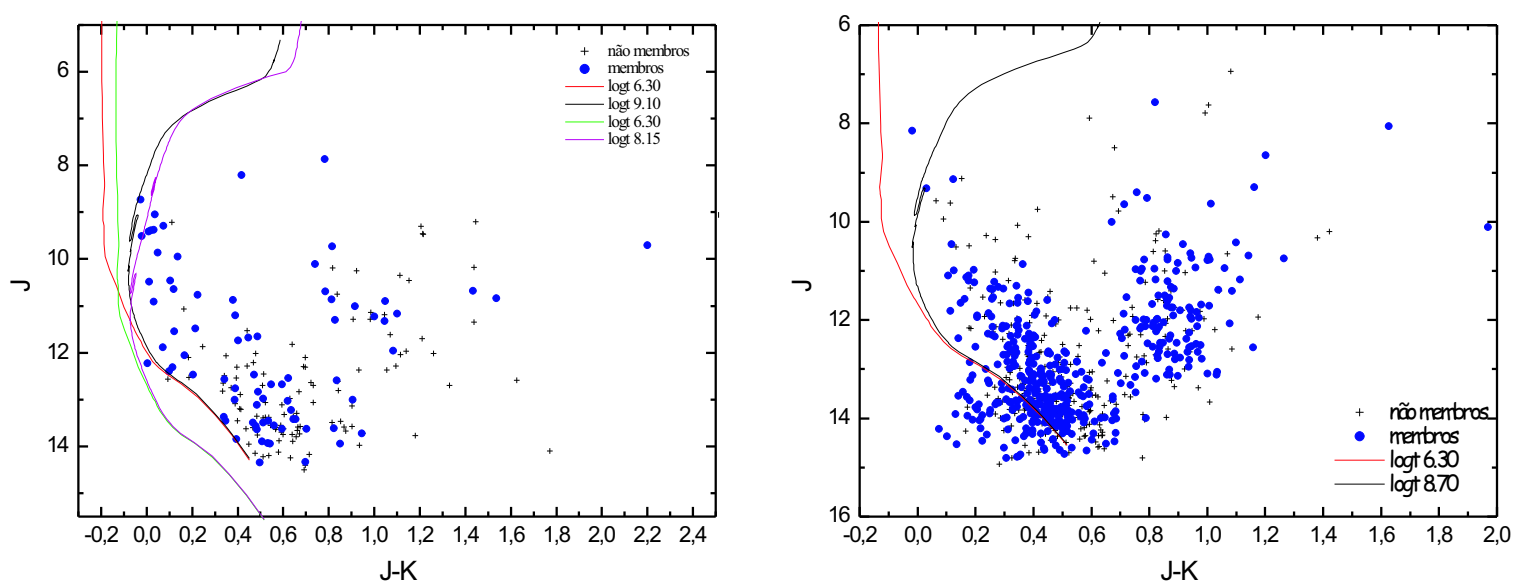

Figura 7.3: Diagramas CMD dos aglomerados Loden 2313, Loden 306, Loden 189, Harvard 16, Eso 332-08 e Collinder 62 nas bandas $J-K \times J$ do infravermelho. Nos diagramas dos aglomerados temos o ajuste das isócronas à sequência principal. Em vermelho e preto as isócronas cujo ajuste foi feito neste trabalho e em verde e rosa as isócronas ajustadas com os valores do DAML02 para os casos que possuem valores catalogados na literatura. As isócronas ajustadas possuem metalicidade solar de acordo com os modelos de Padova. 

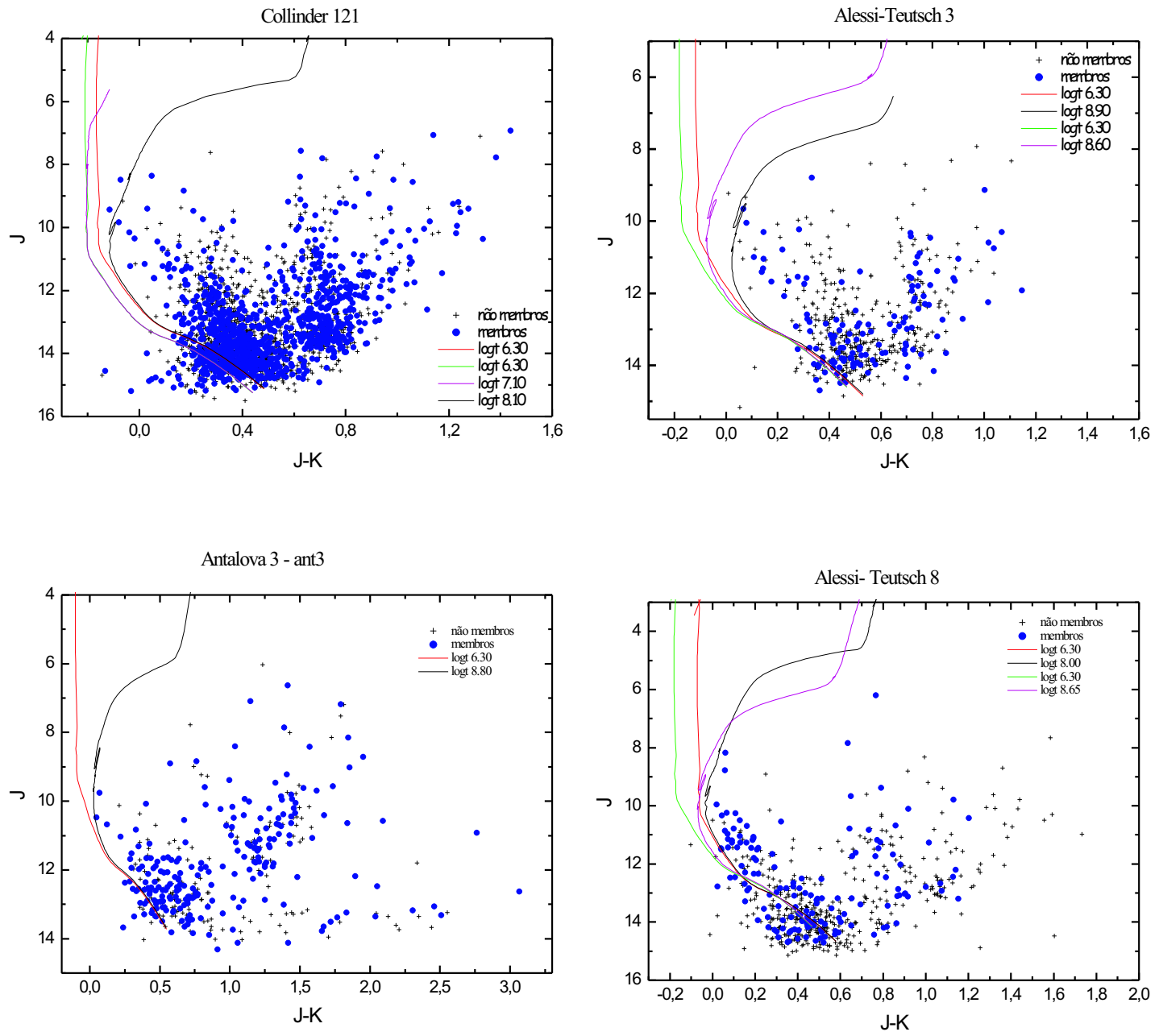

Alessi 37
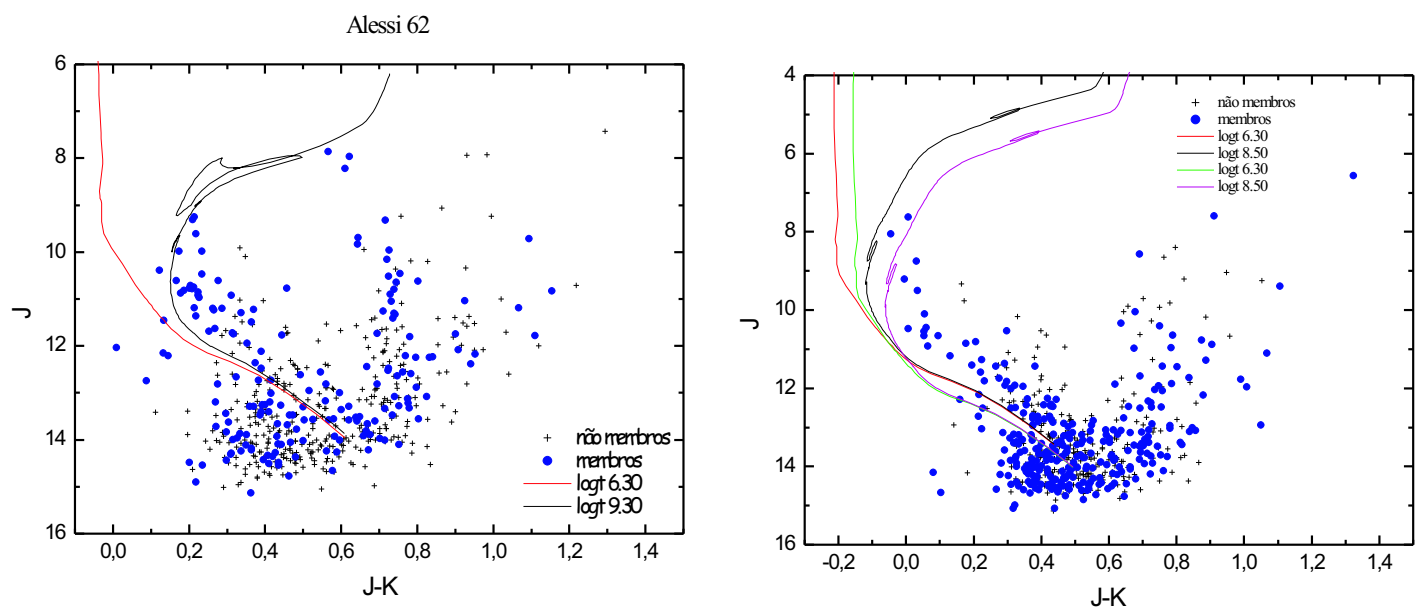

Figura 7.4: Diagramas CMD dos aglomerados Collinder 121, Alessi-Teutsch 3, Antalova 3, Alessi-Teutsch 8, Alessi 62 e Alessi 37 nas bandas $J-K \times J$ do infravermelho. Nos diagramas dos aglomerados temos o ajuste das isócronas à sequência principal. Em vermelho e preto as isócronas cujo ajuste foi feito neste trabalho e em verde e rosa as isócronas ajustadas com os valores do DAML02 para os casos que possuem valores catalogados na literatura. As isócronas ajustadas possuem metalicidade solar de acordo com os modelos de Padova. 

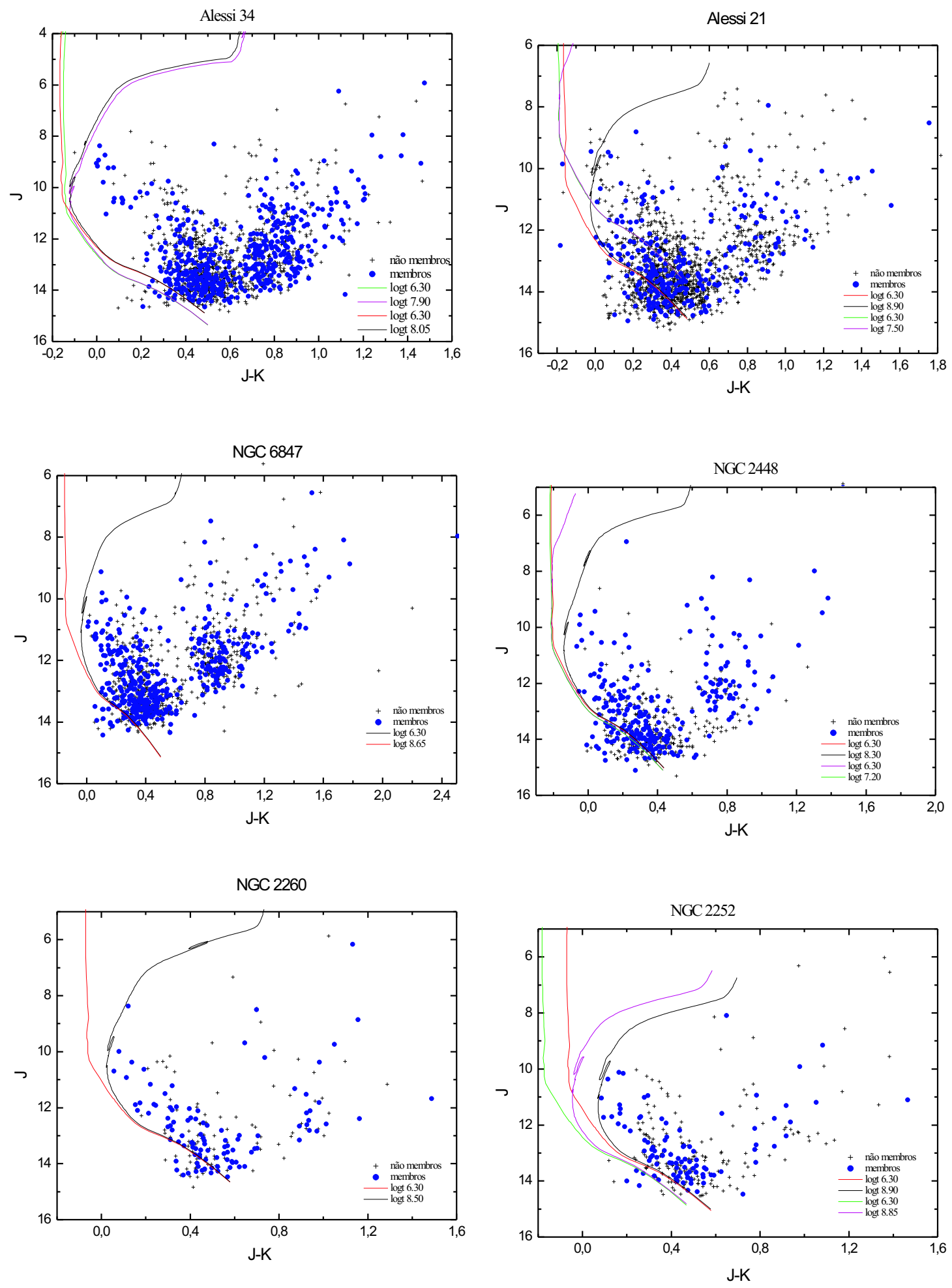

Figura 7.5: Diagramas CMD dos aglomerados Alessi 34, Alessi 21, NGC 6847, NGC 2448, NGC 2260 e NGC 2252 nas bandas $J-K \times J$ do infravermelho. Nos diagramas dos aglomerados temos o ajuste das isócronas à sequência principal. Em vermelho e preto as isócronas cujo ajuste foi feito neste trabalho e em verde e rosa as isócronas ajustadas com os valores do DAML02 para os casos que possuem valores catalogados na literatura. As isócronas ajustadas possuem metalicidade solar de acordo com os modelos de Padova. 

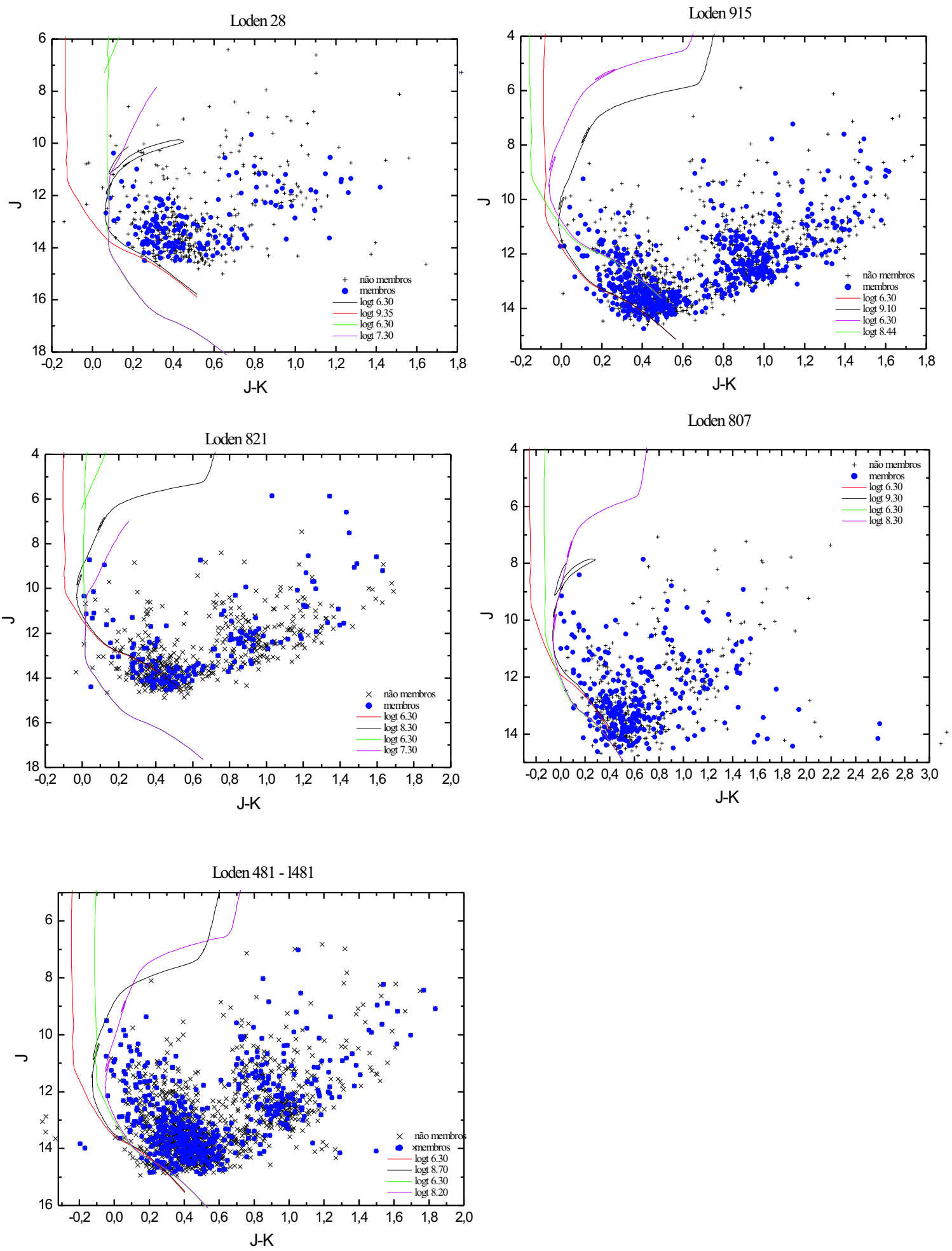

Figura 7.6: Diagramas CMD dos aglomerados Loden 28, Loden 915, Loden 821, Loden 807 e Loden 481 nas bandas $J-K \times J$ do infravermelho. Nos diagramas dos aglomerados temos o ajuste das isócronas à sequência principal. Em vermelho e preto as isócronas cujo ajuste foi feito neste trabalho e em verde e rosa as isócronas ajustadas com os valores do DAML02 para os casos que possuem valores catalogados na literatura. As isócronas ajustadas possuem metalicidade solar de acordo com os modelos de Padova. 

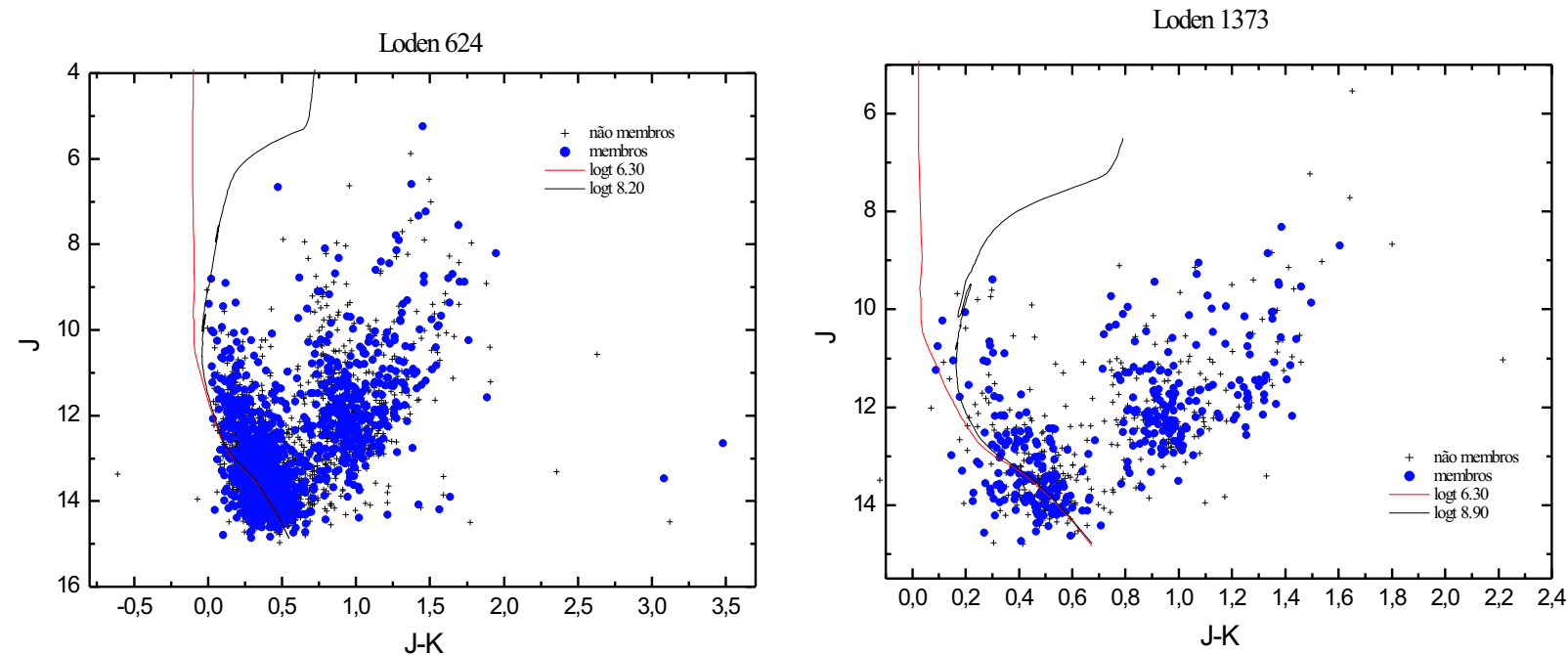

Figura 7.7: Diagramas CMD dos aglomerados Loden 624 e Loden 1373 nas bandas $J-K$ x $J$ do infravermelho. Nos diagramas dos aglomerados temos o ajuste das isócronas à sequência principal. Em vermelho e preto as isócronas cujo ajuste foi feito neste trabalho. As isócronas ajustadas possuem metalicidade solar de acordo com os modelos de Padova. 

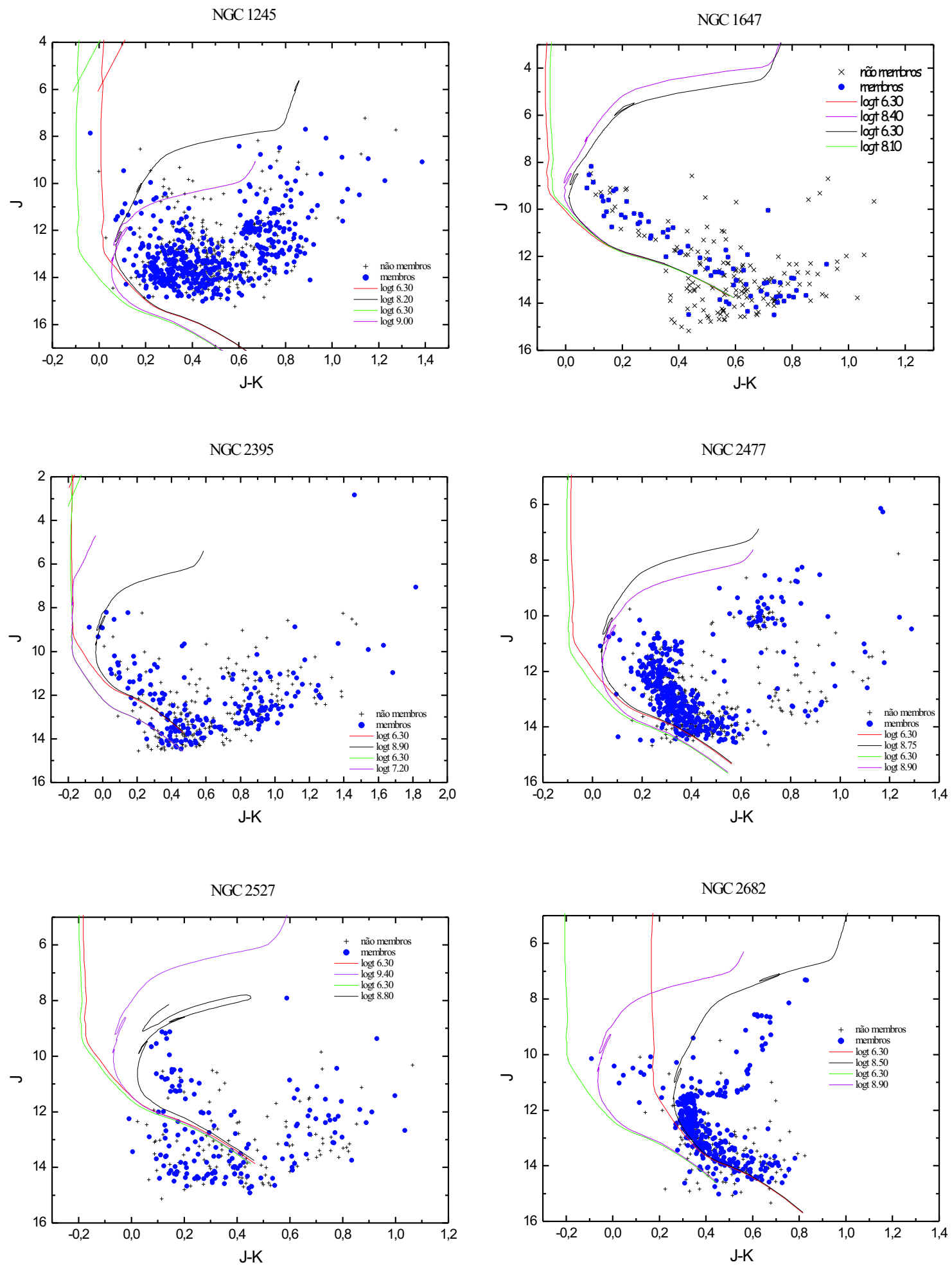

Figura 7.8: Casos em comum com Pauzen - Diagramas CMD dos aglomerados NGC 1245, NGC 1647, NGC 2395, NGC 2477, NGC 2527 e NGC 2682 nas bandas $J-K \times J$ do infravermelho. Nos diagramas dos aglomerados temos o ajuste das isócronas à sequência principal. Em vermelho e preto as isócronas cujo ajuste foi feito neste trabalho e em verde e rosa as isócronas ajustadas com os valores do Pauzen e Netopil[34] para os casos que possuem valores catalogados na literatura. As isócronas ajustadas possuem metalicidade solar de acordo com os modelos de Padova. 

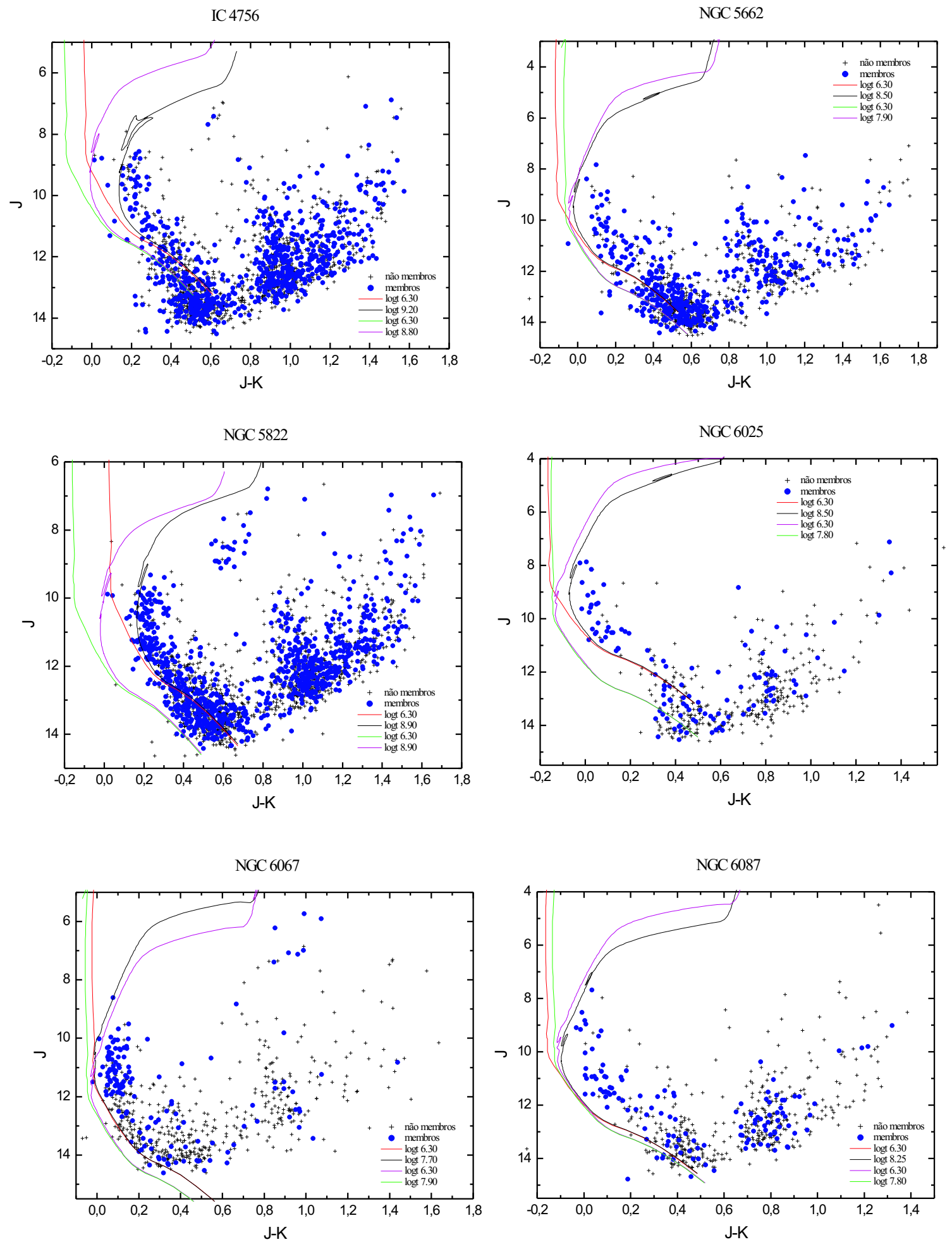

Figura 7.9: Casos em comum com Pauzen - Diagramas CMD dos aglomerados IC 4756, NGC 5662, NGC 5822, NGC 6025, NGC 6067 e NGC 6087 nas bandas $J-K \times J$ do infravermelho. Nos diagramas dos aglomerados temos o ajuste das isócronas à sequência principal. Em vermelho e preto as isócronas cujo ajuste foi feito neste trabalho e em verde e rosa as isócronas ajustadas com os valores do Pauzen e Netopil[34] para os casos que possuem valores catalogados na literatura. As isócronas ajustadas possuem metalicidade solar de acordo com os modelos de Padova. 
NGC 6250
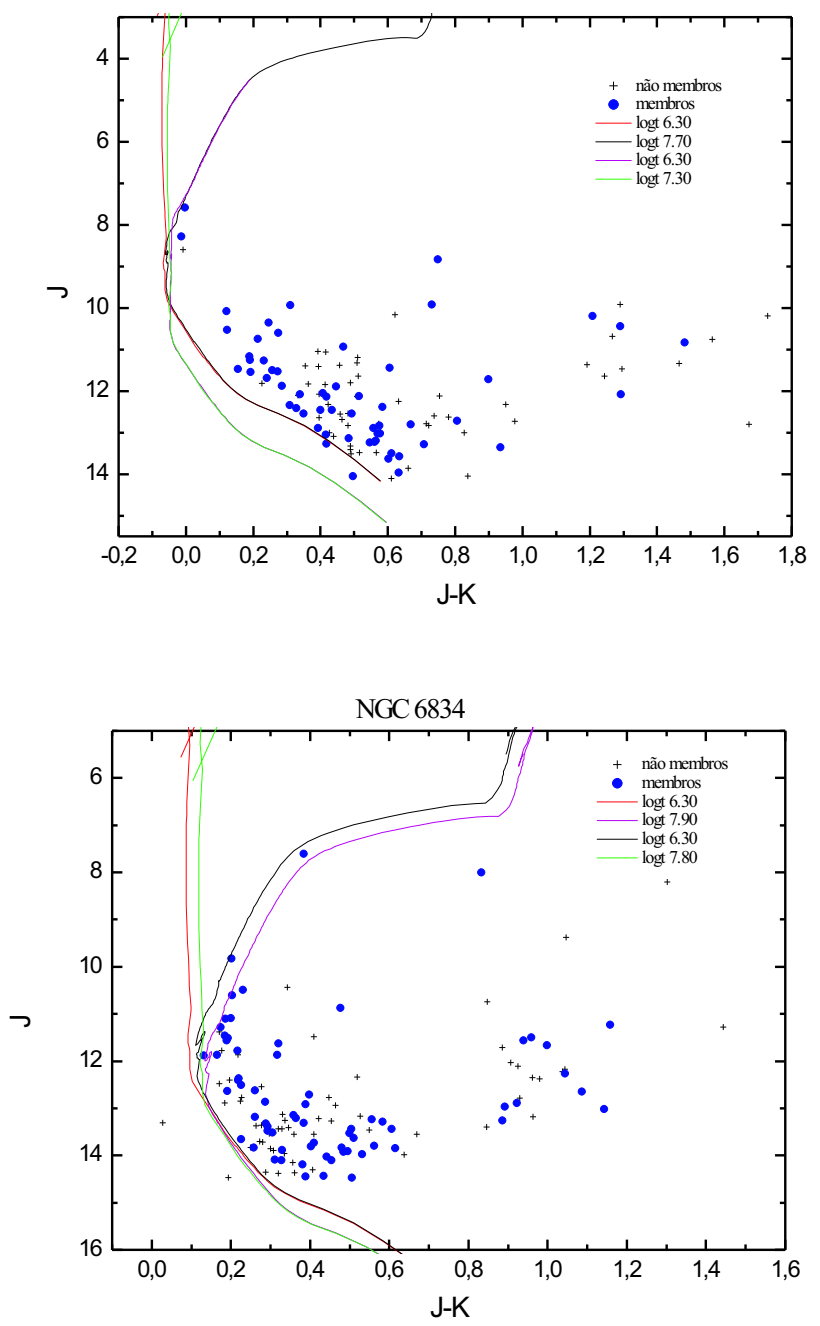

Bochum 10

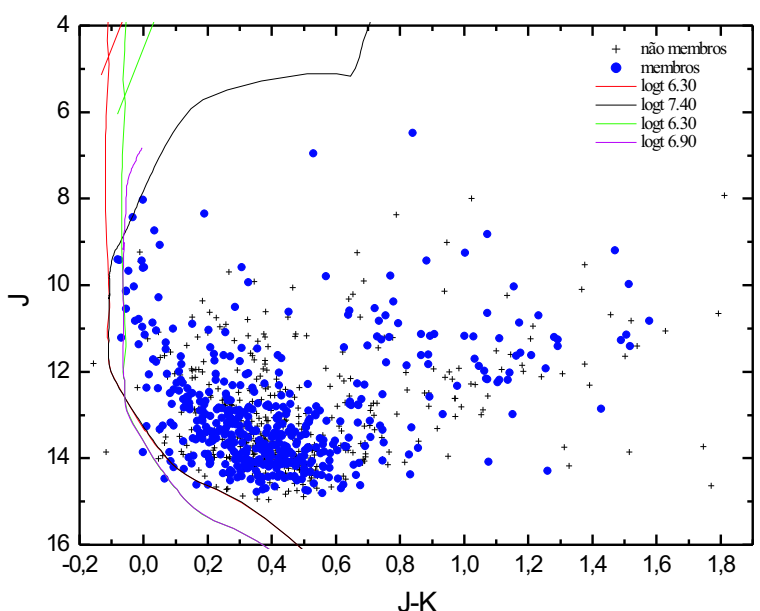

NGC 6405

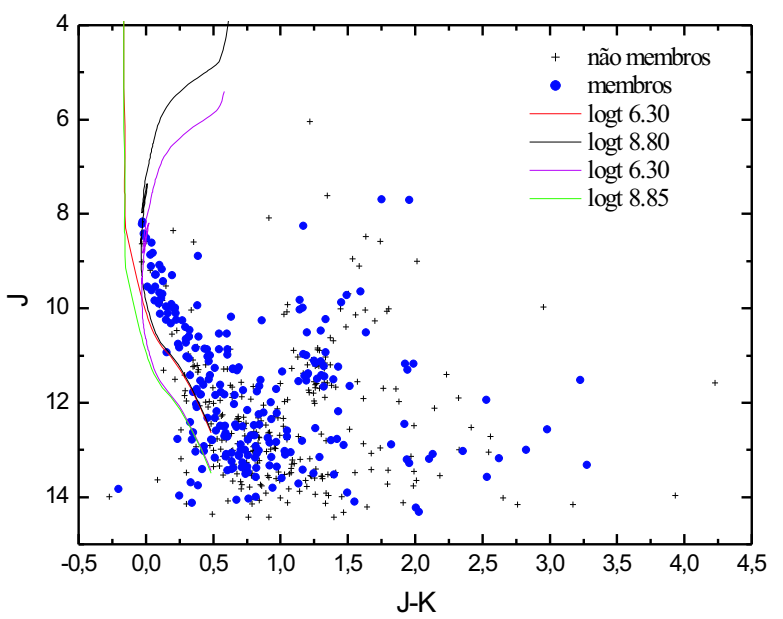

NGC 6871

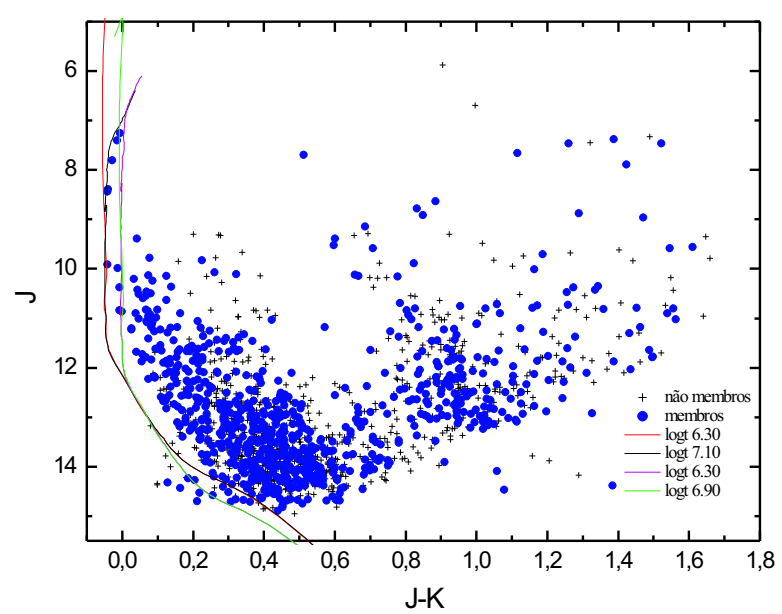

Collinder 394

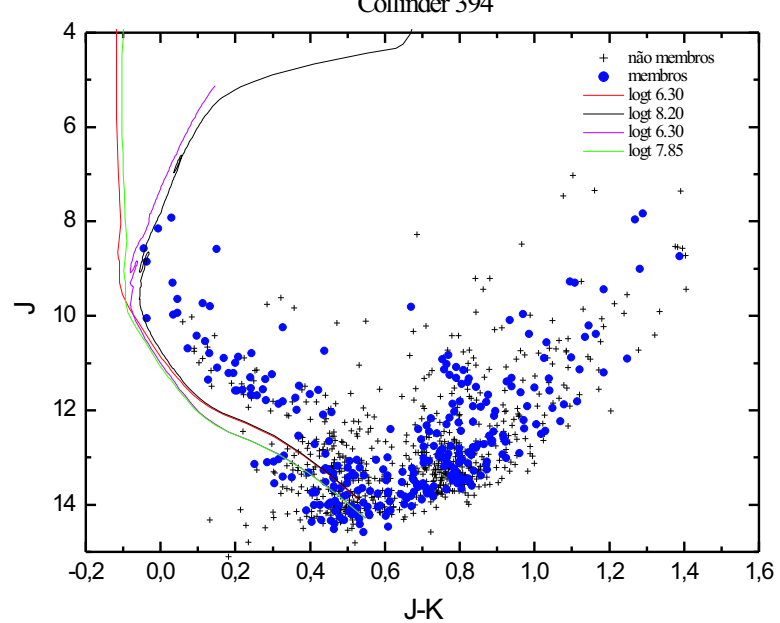

Figura 7.10: Casos em comum com Pauzen - Diagramas CMD dos aglomerados NGC 6250, NGC 6405, NGC 6834, NGC 6871, Bochum 10 e Collinder 394 nas bandas $J-K \times J$ do infravermelho. Nos diagramas dos aglomerados temos o ajuste das isócronas à sequência principal. Em vermelho e preto as isócronas cujo ajuste foi feito neste trabalho e em verde e rosa as isócronas ajustadas com os valores do Pauzen e Netopil[34] para os casos que possuem valores catalogados na literatura. As isócronas ajustadas possuem metalicidade solar de acordo com os modelos de Padova. 
Stock 14

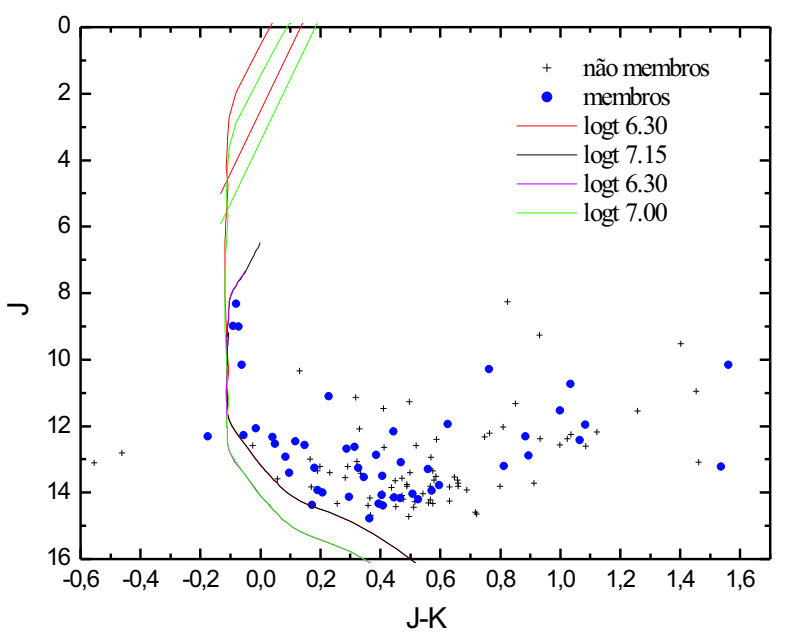

trumpler 10

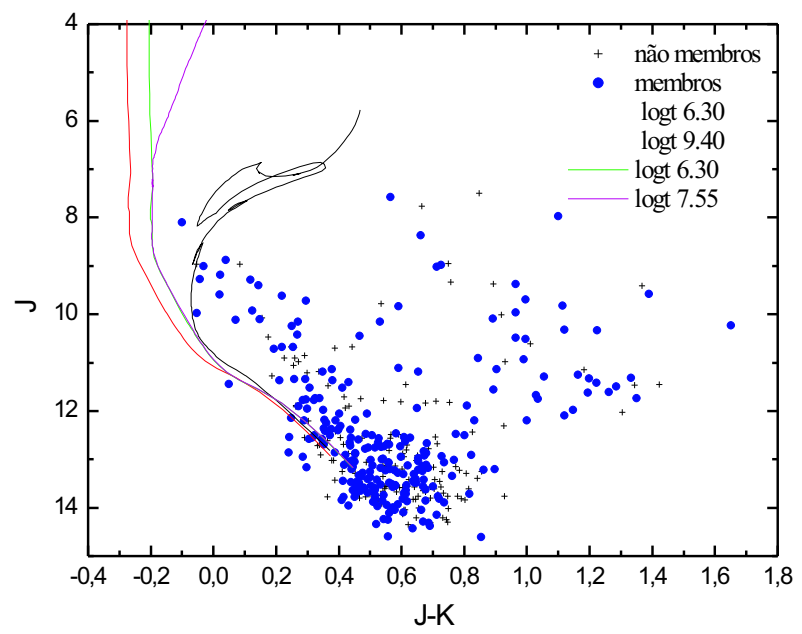

Figura 7.11: Casos em comum com Pauzen - Diagramas CMD dos aglomerados Trumpler 10 e Stock 14 nas bandas $J-K \times J$ do infravermelho. Nos diagramas dos aglomerados temos o ajuste das isócronas à sequência principal. Em vermelho e preto as isócronas cujo ajuste foi feito neste trabalho e em verde e rosa as isócronas ajustadas com os valores do Pauzen e Netopil[34] para os casos que possuem valores catalogados na literatura. As isócronas ajustadas possuem metalicidade solar de acordo com os modelos de Padova. 


\section{Tabela A}

Tabela A: Resultados das análises de movimentos próprios, onde $N_{e}$ é o número de estrelas do aglomerado, $N i$ o número de estrelas intrusas ao aglomerado, $\mu \alpha \cos \delta$ e $\mu \delta$ são as componentes do movimento próprio em milisegundos de arco (mas/yr), $\sigma$ é a dispersão do movimento próprio das estrelas do aglomerado (UCAC2), e $\sigma_{\mu \alpha \cos \delta}$ e $\sigma_{\mu \delta}$ são a dispersão das componentes de movimento próprio das estrelas de campo (DAML02).

\begin{tabular}{|l|c|c|c|c|c|c|c|c|c|}
\hline \multicolumn{1}{|c|}{ Aglomerado } & $\begin{array}{l}\mu \alpha \cos \delta \\
(\operatorname{mas} / y r)\end{array}$ & $\begin{array}{l}\mu \delta \\
(\operatorname{mas} / y r)\end{array}$ & $\sigma$ & $N e$ & $\begin{array}{l}\mu \alpha \cos \delta \\
(\operatorname{mas} / y r)\end{array}$ & $\sigma_{\mu \alpha \cos \delta}$ & $\begin{array}{l}\mu \delta \\
(m a s / y r)\end{array}$ & $\sigma_{\mu \delta}$ & $N f$ \\
\hline NGC 1520 & -1.11 & 4.18 & 2.46 & 7 & 7.32 & 6.58 & 0.18 & 17.84 & 7 \\
\hline NGC 1548 & 2.54 & -3.44 & 3.15 & 70 & 5.28 & 7.12 & -8.85 & 8.49 & 150 \\
\hline NGC 1662 & -0.74 & -1.87 & 2.03 & 70 & 1.56 & 5.77 & -8.14 & 6.98 & 72 \\
\hline NGC 1798 & -1.20 & -4.78 & 3.04 & 20 & -4.12 & 5.62 & -8.58 & 4.07 & 26 \\
\hline NGC 1802 & -0.88 & -3.01 & 2.69 & 140 & 0.50 & 7.97 & -5.65 & 8.12 & 211 \\
\hline NGC 1807 & 1.43 & -5.23 & 3.17 & 40 & 2.46 & 6.17 & -8.23 & 11.56 & 68 \\
\hline NGC 1857 & -3.59 & -4.93 & 2.92 & 60 & -2.71 & 5.84 & -5.87 & 5.11 & 81 \\
\hline NGC 1896 & 0.55 & -5.01 & 3.17 & 100 & 1.86 & 10.41 & -7.82 & 10.86 & 187 \\
\hline NGC 1996 & -0.33 & -2.47 & 2.46 & 110 & 1.59 & 5.57 & -5.56 & 6.77 & 122 \\
\hline NGC 2026 & -1.16 & -3.68 & 2.57 & 30 & 0.15 & 7.33 & -7.24 & 8.26 & 50 \\
\hline NGC 2061 & 0.54 & -2.14 & 2.80 & 15 & 1.11 & 5.70 & 10.51 & 8.82 & 17 \\
\hline NGC 2156 & -2.61 & -2.08 & 1.63 & 12 & -3.47 & 7.73 & 1.59 & 13.43 & 10 \\
\hline NGC 2192 & -0.69 & -5.52 & 3.05 & 20 & -0.98 & 18.23 & -6.01 & 17.40 & 34 \\
\hline NGC 2202 & 0.42 & -4.11 & 3.56 & 15 & 3.93 & 11.46 & -6.89 & 10.60 & 39 \\
\hline NGC 2224 & -0.94 & -1.39 & 3.30 & 120 & -1.76 & 6.61 & -2.50 & 6.53 & 160 \\
\hline NGC 2234 & -0.72 & -6.94 & 3.36 & 350 & -0.63 & 7.93 & -9.45 & 8.19 & 825 \\
\hline NGC 2252 & -1.08 & -1.57 & 3.05 & 75 & -2.95 & 6.02 & -3.72 & 8.94 & 198 \\
\hline NGC 2260 & -0.49 & -0.69 & 3.04 & 80 & 0.72 & 5.26 & -2.99 & 7.49 & 124 \\
\hline NGC 2262 & -1.31 & -0.79 & 3.17 & 7 & -5.00 & 15.87 & 0.12 & 11.57 & 37 \\
\hline NGC 2265 & -0.03 & -5.03 & 2.13 & 30 & 0.74 & 7.14 & -6.40 & 9.64 & 53 \\
\hline NGC 2306 & -5.19 & -1.72 & 3.17 & 31 & -1.05 & 7.33 & -3.41 & 5.79 & 120 \\
\hline NGC 2319 & -2.00 & -2.14 & 3.04 & 45 & -1.17 & 6.86 & -3.34 & 7.37 & 204 \\
\hline NGC 2331 & -0.71 & -1.89 & 2.69 & 60 & -2.08 & 5.32 & -5.92 & 7.12 & 85 \\
\hline NGC 2351 & -3.99 & 1.12 & 2.94 & 10 & -5.96 & 9.05 & 1.70 & 11.73 & 18 \\
\hline NGC 2352 & -2.25 & 3.03 & 3.04 & 30 & -4.56 & 6.33 & 1.35 & 8.22 & 40 \\
\hline NGC 2364 & -2.83 & 0.92 & 3.05 & 11 & -3.16 & 9.53 & -0.49 & 10.19 & 49 \\
\hline NGC 2447 & -5.12 & 3.62 & 2.80 & 90 & -6.50 & 10.20 & 0.67 & 10.88 & 172 \\
\hline NGC 2448 & -2.34 & 2.86 & 3.04 & 205 & -4.31 & 7.04 & 1.61 & 6.22 & 319 \\
\hline
\end{tabular}




\begin{tabular}{|c|c|c|c|c|c|c|c|c|c|}
\hline \multicolumn{5}{|c|}{ MEMBROS } & \multicolumn{5}{|c|}{ Campo } \\
\hline Aglomerado & $\begin{array}{l}\mu \alpha \cos \delta \\
(\operatorname{mas} / y r)\end{array}$ & $\begin{array}{c}\mu \delta \\
(\operatorname{mas} / y r)\end{array}$ & $\sigma$ & $\mathrm{Ne}$ & $\begin{array}{l}\mu \alpha \cos \delta \\
(\operatorname{mas} / y r)\end{array}$ & $\sigma_{\mu \alpha \cos \delta}$ & $\begin{array}{c}\mu \delta \\
(\operatorname{mas} / y r)\end{array}$ & $\sigma_{\mu \delta}$ & $\mathrm{Nf}$ \\
\hline NGC 2455 & -6.82 & 3.33 & 2.92 & 20 & -5.93 & 12.47 & 6.71 & 4.42 & 56 \\
\hline C 2849 & -6.74 & 4.85 & 2.71 & 12 & -11.92 & 11.43 & 5.34 & 10.28 & 50 \\
\hline NGC 2982 & -11.90 & 6.27 & 2.92 & 23 & -8.32 & 7.65 & 3.62 & 8.86 & 27 \\
\hline NGC 3036 & -4.82 & 4.62 & 2.80 & 20 & -10.35 & 7.15 & 0.92 & 5.43 & 28 \\
\hline NGC 3446 & -7.84 & 2.00 & 2.93 & 23 & -9.29 & 12.24 & 2.24 & 10.30 & 96 \\
\hline NGC 3909 & -8.54 & 2.76 & 2.92 & 32 & -9.85 & 10.56 & 1.95 & 8.16 & 100 \\
\hline NGC 4230 & -11.38 & -1.36 & 2.92 & 15 & -7.34 & 10.73 & -0.35 & 9.37 & 79 \\
\hline NGC 5043 & -6.54 & 0.91 & 2.94 & 46 & -7.81 & 10.36 & 0.50 & 10.61 & 285 \\
\hline NGC 5138 & -5.80 & 0.15 & 2.90 & 32 & -10.15 & 9.76 & -0.29 & 12.03 & 99 \\
\hline NGC 5269 & -7.94 & -2.32 & 2.69 & 10 & -7.87 & 10.94 & -2.02 & 11.97 & 32 \\
\hline NGC 5359 & -5.22 & -4.94 & 3.04 & 86 & -6.93 & 8.81 & -6.29 & 8.55 & 275 \\
\hline NGC5381 & -4.51 & -1.51 & 3.04 & 50 & -4.37 & 9.75 & -3.40 & 9.65 & 239 \\
\hline NGC 5715 & -4.79 & -2.77 & 3.04 & 30 & -7.37 & 10.52 & -5.23 & 10.45 & 130 \\
\hline NGC 5764 & -6.55 & -5.41 & 2.80 & 15 & -5.94 & 7.99 & -7.03 & 9.24 & 20 \\
\hline NGC 5800 & -8.47 & -2.56 & 3.02 & 40 & -7.60 & 8.42 & -5.66 & 7.30 & 149 \\
\hline NGC 5925 & -2.69 & -4.90 & 2.93 & 165 & -4.04 & 9.35 & -5.52 & 8.47 & 478 \\
\hline NGC 5998 & -0.86 & -0.16 & 3.20 & 8 & -1.50 & 8.48 & -5.68 & 6.68 & 11 \\
\hline NGC 6169 & -2.40 & -3.78 & 2.91 & 32 & -3.61 & 7.46 & -1.54 & 7.91 & 153 \\
\hline NGC 6334 & 0.02 & -1.89 & 2.81 & 130 & 0.001 & 5.75 & -5.11 & 6.14 & 144 \\
\hline NGC 6554 & -0.36 & -0.04 & 2.92 & 40 & 0.74 & 8.23 & 1.01 & 7.28 & 378 \\
\hline NGC 6639 & -0.86 & -0.53 & 2.92 & 16 & 0.55 & 14.24 & -3.94 & 15.58 & 57 \\
\hline NGC 6645 & 0.56 & -6.04 & 3.04 & 40 & 1.80 & 21.09 & -6.10 & 21.01 & 531 \\
\hline NGC 6698 & -1.36 & -4.02 & 3.04 & 21 & -6.77 & 9.28 & -5.28 & 8.28 & 157 \\
\hline NGC 6735 & -1.24 & -4.20 & 2.93 & 80 & -1.52 & 7.20 & -1.82 & 7.18 & 195 \\
\hline NGC 6738 & -0.22 & -4.38 & 2.93 & 51 & 2.01 & 6.70 & -3.60 & 8.56 & 225 \\
\hline NGC 6743 & 1.72 & -2.09 & 2.57 & 15 & -0.96 & 8.76 & -3.00 & 9.05 & 32 \\
\hline NGC 6773 & -2.98 & -6.55 & 2.80 & 18 & -1.24 & 9.49 & -3.20 & 8.98 & 73 \\
\hline NGC 6791 & -2.45 & -0.32 & 1.72 & 23 & 0.06 & 9.42 & -1.85 & 13.94 & 102 \\
\hline NGC 6827 & -3.18 & -4.10 & 2.80 & 14 & -5.30 & 3.47 & -10.25 & 8.90 & 13 \\
\hline NGC 6828 & -2.49 & -5.06 & 2.81 & 16 & -1.20 & 12.24 & -5.32 & 12.95 & 80 \\
\hline NGC 6839 & -1.18 & -4.75 & 2.81 & 20 & -0.92 & 10.63 & -4.73 & 10.06 & 58 \\
\hline NGC 6847 & -1.37 & -3.88 & 3.04 & 300 & -1.09 & 9.51 & -4.00 & 10.78 & 770 \\
\hline NGC 6858 & 1.16 & -2.91 & 2.92 & 21 & 1.42 & 6.64 & -4.85 & 6.57 & 102 \\
\hline NGC 6873 & -3.63 & -7.06 & 2.80 & 50 & -2.41 & 9.12 & -6.13 & 9.44 & 172 \\
\hline NGC 6904 & -2.16 & -7.82 & 3.04 & 10 & 0.81 & 9.82 & -3.55 & 6.25 & 74 \\
\hline NGC 6938 & -0.42 & -4.21 & 2.92 & 18 & 0.60 & 8.72 & -4.92 & 8.77 & 50 \\
\hline NGC 6950 & -3.34 & -4.01 & 3.05 & 21 & -0.81 & 6.68 & -3.22 & 6.09 & 120 \\
\hline NGC 7084 & -1.60 & -8.45 & 3.17 & 25 & 0.95 & 7.87 & -10.86 & 9.18 & 60 \\
\hline $\begin{array}{l}\text { NGC } 7093 \\
\end{array}$ & -2.29 & -4.15 & 3.06 & 45 & -2.57 & 9.34 & -4.74 & 7.84 & 108 \\
\hline NGC 7686 & 0.46 & -8.99 & 3.05 & 55 & 1.59 & 11.66 & -8.48 & 8.95 & 114 \\
\hline$N G C 7801$ & -2.88 & -6.05 & 3.04 & 20 & 0.82 & 8.21 & -4.37 & 5.28 & 47 \\
\hline
\end{tabular}




\begin{tabular}{|c|c|c|c|c|c|c|c|c|c|}
\hline \multicolumn{5}{|c|}{ MEMBROS } & \multicolumn{5}{|c|}{ Campo } \\
\hline Aglomerado & $\begin{array}{l}\mu \alpha \cos \delta \\
(\mathrm{mas} / \mathrm{yr})\end{array}$ & $\begin{array}{c}\mu \delta \\
(\operatorname{mas} / \gamma r)\end{array}$ & $\sigma$ & $\mathrm{Ne}$ & $\begin{array}{l}\mu \alpha \cos \delta \\
(\operatorname{mas} / y r)\end{array}$ & $\sigma_{\mu \alpha \cos \delta}$ & $\begin{array}{c}\mu \delta \\
(\mathrm{mas} / \mathrm{yr})\end{array}$ & $\sigma_{\mu \delta}$ & $\mathrm{Nf}$ \\
\hline H03 J2011+26.7 & -1.03 & -3.31 & 2.80 & 9 & 0.73 & 14.51 & -3.59 & 16.47 & 23 \\
\hline AH03 J0748-6.9 & -2.78 & 2.98 & 2.80 & 14 & -4.84 & 7.46 & 2.47 & 7.93 & 22 \\
\hline AH03 J0822-36.4 & -4.24 & 5.59 & 3.04 & 85 & -6.26 & 7.43 & 3.89 & 7.86 & 110 \\
\hline Alessi 10 & 0.84 & -3.97 & 3.17 & 40 & 3.42 & 7.83 & -8.45 & 5.45 & 109 \\
\hline Alessi 21 & -0.27 & -1.33 & 2.93 & 281 & 0.02 & 7.75 & -2.56 & 7.08 & 1249 \\
\hline Alessi 22 & 0.25 & -3.85 & 3.04 & 300 & 3.24 & 13.01 & -5.79 & 10.02 & 1096 \\
\hline Alessi 24 & -1.03 & -5.70 & 3.17 & 1000 & -0.80 & 6.39 & -7.70 & 7.91 & 5089 \\
\hline Alessi 34 & -3.28 & 4.51 & 2.93 & 370 & -5.06 & 8.54 & 5.46 & 9.72 & 972 \\
\hline Alessi 37 & 0.51 & -1.08 & 3.02 & 250 & 1.52 & 8.82 & -1.92 & 8.75 & 300 \\
\hline Alessi 44 & -0.86 & -5.95 & 3.06 & 600 & 0.89 & 5.40 & -4.65 & 5.37 & 1634 \\
\hline Alessi 62 & -1.04 & -1.09 & 2.81 & 125 & -1.94 & 10.00 & -4.93 & 10.38 & 407 \\
\hline Alessi 9 & 2.08 & -6.66 & 3.36 & 130 & 3.43 & 10.97 & -9.43 & 10.32 & 231 \\
\hline Alessi-Teutsch 8 & -4.80 & 1.14 & 3.05 & 125 & -6.63 & 9.25 & 0.06 & 8.22 & 451 \\
\hline Antalova 3 & 1.57 & -1.79 & 2.69 & 186 & 0.93 & 6.71 & -6.05 & 8.19 & 199 \\
\hline Alessi-Teutsch 12 & 0.48 & -2.99 & 3.07 & 250 & -0.11 & 8.19 & -5.03 & 9.80 & 1123 \\
\hline Alessi-Teutsch 3 & -2.19 & 6.60 & 2.92 & 110 & -3.06 & 6.67 & 6.82 & 8.94 & 373 \\
\hline Alessi-Teutsch 7 & -6.47 & 3.56 & 2.82 & 90 & -8.33 & 7.84 & 4.79 & 7.91 & 255 \\
\hline BH 150 & -5.14 & -3.33 & 2.80 & 33 & -7.81 & 9.69 & -3.32 & 5.99 & 72 \\
\hline BH 197 & -1.66 & -5.77 & 2.69 & 22 & -2.37 & 7.52 & -6.54 & 10.43 & 30 \\
\hline BH 202 & -3.32 & -2.47 & 2.93 & 17 & -0.36 & 15.53 & -4.01 & 14.98 & 109 \\
\hline BH 211 & -1.64 & -0.83 & 2.35 & 17 & -3.24 & 6.75 & -5.93 & 6.70 & 25 \\
\hline $\mathrm{BH} 223$ & -0.21 & -2.22 & 2.82 & 18 & -0.81 & 6.82 & -3.52 & 3.14 & 24 \\
\hline BH 231 & 0.40 & -4.37 & 2.69 & 13 & -0.79 & 13.76 & -6.25 & 12.99 & 14 \\
\hline Berkeley 15 & 1.09 & -6.44 & 2.93 & 40 & 0.53 & 6.45 & -7.19 & 3.53 & 37 \\
\hline Berkeley 25 & -2.60 & -0.65 & 3.17 & 7 & 8.59 & 33.13 & 17.96 & 26.62 & 8 \\
\hline Berkeley 77 & -6.79 & -0.07 & 2.80 & 20 & -6.17 & 5.32 & 0.79 & 6.03 & 49 \\
\hline Berkeley 80 & -2.18 & -6.09 & 2.69 & 13 & -1.00 & 11.09 & -1.68 & 13.66 & 13 \\
\hline Berkeley 85 & -0.75 & 0.29 & 2.80 & 65 & -2.29 & 13.01 & -3.42 & 10.54 & 122 \\
\hline BH54 & -5.27 & 6.59 & 2.92 & 13 & -5.94 & 9.36 & 0.33 & 18.48 & 18 \\
\hline $\mathrm{BH} 72$ & -6.73 & 4.42 & 2.92 & 7 & -4.30 & 9.64 & 3.51 & 10.48 & 12 \\
\hline $\mathrm{BH} 84$ & -7.88 & 6.88 & 2.80 & 23 & -5.01 & 14.16 & 8.05 & 15.28 & 28 \\
\hline BH 87 & -10.5 & 4.99 & 3.04 & 5 & -5.63 & 12.75 & 8.76 & 14.57 & 13 \\
\hline Brandt 1 & -1.98 & 4.26 & 3.05 & 120 & -2.84 & 6.51 & 5.52 & 7.75 & 223 \\
\hline Collinder 115 & -0.76 & -4.62 & 3.30 & 63 & -2.70 & 9.53 & -2.82 & 6.47 & 72 \\
\hline Collinder 121 & -3.67 & 1.52 & 2.81 & 800 & -4.83 & 5.78 & 1.94 & 6.78 & 1425 \\
\hline Collinder 187 & -2.36 & 2.64 & 2.31 & 11 & -4.41 & 9.29 & 5.77 & 4.77 & 20 \\
\hline Collinder 198 & -5.11 & -0.35 & 3.02 & 8 & -11.58 & 8.80 & 6.48 & 4.57 & 13 \\
\hline Collinder 213 & -6.36 & 4.99 & 3.04 & 130 & -6.39 & 11.52 & 4.85 & 11.31 & 200 \\
\hline Collinder 257 & -8.92 & 0.02 & 3.04 & 8 & -3.61 & 10.63 & -2.05 & 7.35 & 37 \\
\hline Collinder 269 & -6.31 & -3.90 & 2.80 & 105 & -6.96 & 10.38 & -3.66 & 10.54 & 364 \\
\hline Collinder 307 & -3.62 & -3.67 & 2.80 & 21 & -2.23 & 10.56 & -5.76 & 8.78 & 87 \\
\hline
\end{tabular}




\begin{tabular}{|c|c|c|c|c|c|c|c|c|c|}
\hline \multicolumn{5}{|c|}{ MEMBROS } & \multicolumn{5}{|c|}{ Campo } \\
\hline Aglomerado & $\begin{array}{l}\mu \alpha \cos \delta \\
(\mathrm{mas} / \mathrm{y})\end{array}$ & $\begin{array}{c}\mu \delta \\
(\text { mas } / y r)\end{array}$ & $\sigma$ & $\mathrm{Ne}$ & $\begin{array}{l}\mu \alpha \cos \delta \\
(\operatorname{mas} / y r)\end{array}$ & $\sigma_{\mu \alpha \cos \delta}$ & $\begin{array}{c}\mu \delta \\
(\mathrm{mas} / \mathrm{yr})\end{array}$ & $\sigma_{\mu \delta}$ & Nf \\
\hline Collinder 333 & -6.20 & 0.85 & 2.69 & 30 & -4.57 & 13.28 & -5.41 & 13.20 & 43 \\
\hline Collinder 345 & 0.40 & 0.28 & 2.80 & 20 & 0.74 & 9.34 & -3.93 & 8.67 & 50 \\
\hline Collinder 416 & -4.52 & -4.42 & 2.92 & 48 & -3.45 & 7.56 & -3.05 & 5.31 & 75 \\
\hline Collinder 466 & -2.82 & -7.16 & 2.13 & 10 & -1.72 & 9.26 & 0.27 & 10.12 & 34 \\
\hline Collinder 62 & -1.48 & -2.59 & 3.02 & 340 & -0.19 & 6.24 & -4.82 & 8.23 & 393 \\
\hline Czernik 23 & -0.27 & -4.47 & 2.59 & 18 & -0.36 & 4.50 & 3.66 & 7.92 & 17 \\
\hline Czernik 32 & -2.16 & -3.64 & 2.90 & 7 & -4.68 & 6.98 & -2.38 & 9.36 & 6 \\
\hline Czernik 39 & -1.87 & -7.98 & 2.57 & 27 & 2.19 & 5.23 & -4.90 & 3.97 & 55 \\
\hline Czernik 41 & -0.62 & -5.11 & 2.13 & 15 & 0.55 & 8.43 & 1.07 & 7.89 & 17 \\
\hline Dias 3 & 2.09 & -0.92 & 3.05 & 21 & 0.33 & 10.37 & -3.00 & 12.35 & 139 \\
\hline Dias 4 & -3.66 & -3.01 & 2.57 & 6 & -7.26 & 14.26 & -6.28 & 16.80 & 18 \\
\hline Dol-Dzim 11 & 0.08 & -3.99 & 2.80 & 12 & 4.05 & 14.95 & -3.70 & 13.93 & 16 \\
\hline Dolidze 11 & 1.24 & -3.05 & 2.46 & 7 & -5.86 & 10.66 & -7.31 & 10.07 & 11 \\
\hline Dolidze 15 & 2.05 & 0.01 & 2.57 & 50 & 3.86 & 6.99 & -4.65 & 6.81 & 71 \\
\hline Dolidze 16 & -1.29 & -2.88 & 3.17 & 10 & 4.02 & 11.54 & -8.03 & 7.20 & 18 \\
\hline Dolidze 19 & -4.44 & -3.83 & 3.17 & 35 & -0.64 & 8.02 & -7.32 & 8.42 & 84 \\
\hline Dolidze 20 & -0.24 & -7.56 & 2.81 & 18 & 1.44 & 14.66 & -9.38 & 14.60 & 35 \\
\hline Dolidze 24 & -1.70 & -1.45 & 3.04 & 50 & 0.45 & 9.94 & -3.59 & 10.79 & 134 \\
\hline Dolidze 26 & -2.89 & -1.61 & 2.69 & 140 & -3.22 & 7.57 & -4.23 & 5.53 & 155 \\
\hline Dolidze 36 & -2.19 & -2.31 & 2.92 & 55 & -0.96 & 7.76 & -2.01 & 8.85 & 156 \\
\hline Dolidze 37 & 3.01 & -2.65 & 2.69 & 12 & -4.01 & 10.90 & -5.27 & 9.89 & 50 \\
\hline Dolidze 38 & -1.59 & -2.05 & 3.02 & 130 & -1.42 & 8.61 & -2.61 & 7.88 & 278 \\
\hline Dolidze 40 & -1.12 & -0.73 & 2.90 & 95 & -0.71 & 9.09 & -2.11 & 8.53 & 160 \\
\hline Dolidze 41 & -0.87 & 0.19 & 2.80 & 65 & -1.78 & 12.94 & -2.88 & 9.92 & 123 \\
\hline Dolidze 44 & 1.46 & -0.01 & 2.93 & 15 & 2.17 & 12.54 & -0.71 & 10.43 & 49 \\
\hline Dolidze 45 & -0.73 & -2.32 & 2.80 & 70 & -0.29 & 12.74 & -3.36 & 11.01 & 266 \\
\hline Dolidze 47 & 1.53 & -0.19 & 2.35 & 8 & -2.53 & 11.74 & -2.35 & 9.10 & 25 \\
\hline Dolidze 9 & -0.49 & -2.40 & 2.80 & 55 & -1.11 & 8.76 & -1.93 & 11.92 & 219 \\
\hline Dolidze 1 & -2.40 & -3.88 & 2.94 & 9 & -3.90 & 15.06 & $-3.80^{-}$ & 15.99 & 38 \\
\hline Dolidze 12 & 3.69 & -4.72 & 2.57 & 22 & 1.21 & 6.48 & -9.51 & 10.58 & 21 \\
\hline Dolidze 14 & -0.46 & -4.91 & 2.92 & 80 & 0.50 & 4.15 & -6.92 & 9.65 & 142 \\
\hline Dolidze 2 & -0.58 & -0.60 & 2.92 & 11 & -1.11 & 7.11 & -0.69 & 8.15 & 43 \\
\hline Dolidze 3 & -0.58 & -3.13 & 2.80 & 22 & -1.47 & 9.69 & -1.68 & 8.19 & 59 \\
\hline Dolidze 4 & -2.26 & -3.54 & 2.92 & 5 & -4.84 & 14.21 & 1.93 & 10.99 & 9 \\
\hline Dolidze 5 & -4.06 & -6.11 & 2.92 & 20 & -0.70 & 11.19 & -4.44 & 11.77 & 90 \\
\hline ESO 008-06 & -6.60 & -4.56 & 3.04 & 7 & -6.12 & 12.37 & -3.60 & 12.42 & 45 \\
\hline ESO 021-06 & -6.72 & -2.85 & 3.05 & 10 & -2.82 & 11.74 & -4.74 & 11.41 & 61 \\
\hline ESO $065-03$ & -6.53 & -1.70 & 2.80 & 25 & -7.52 & 8.19 & -2.87 & 6.76 & 70 \\
\hline ESO $065-07$ & -6.84 & -0.84 & 2.93 & 13 & -10.90 & 11.34 & -8.21 & 11.19 & 36 \\
\hline ESO 092-05 & -8.41 & 4.63 & 3.02 & 16 & -5.13 & 6.16 & 2.49 & 10.97 & 39 \\
\hline
\end{tabular}




\begin{tabular}{|c|c|c|c|c|c|c|c|c|c|}
\hline \multicolumn{5}{|c|}{ MEMBROS } & \multicolumn{5}{|c|}{ Campo } \\
\hline Aglomerado & $\begin{array}{l}\mu \alpha \cos \delta \\
(\mathrm{mas} / \mathrm{yr})\end{array}$ & $\begin{array}{c}\mu \delta \\
(\mathrm{mas} / \mathrm{yr})\end{array}$ & $\sigma$ & $\mathrm{Ne}$ & $\begin{array}{l}\mu \alpha \cos \delta \\
(\operatorname{mas} / y r)\end{array}$ & $\sigma_{\mu x \cos \delta}$ & $\begin{array}{c}\mu \delta \\
(\operatorname{mas} / \gamma r)\end{array}$ & $\sigma_{\mu \delta}$ & $\mathrm{Nf}$ \\
\hline ESO 097-15 & -5.97 & -4.45 & 2.92 & 65 & -7.55 & 13.54 & -3.69 & 13.95 & 306 \\
\hline ESO 129-32 & -4.67 & -0.48 & 2.92 & 27 & -5.98 & 11.15 & 1.57 & 9.108 & 144 \\
\hline ESO 123-26 & -1.48 & 8.14 & 3.05 & 39 & -1.89 & 6.69 & 7.81 & 7.967 & 130 \\
\hline ESO 128-16 & -3.24 & 7.45 & 2.90 & 70 & -5.30 & 9.15 & 5.94 & 9.850 & 171 \\
\hline ESO 129-19 & -7.26 & 0.30 & 2.95 & 10 & -9.15 & 11.58 & -1.55 & 10.34 & 68 \\
\hline ESO 130-06 & -8.83 & 1.26 & 2.69 & 70 & -8.49 & 9.25 & 0.65 & 8.32 & 139 \\
\hline ESO 131-09 & -7.18 & -2.07 & 2.92 & 10 & -12.70 & 14.72 & -2.01 & 6.67 & 50 \\
\hline ESO 134-12 & -1.19 & -3.75 & 2.93 & 7 & -0.48 & 12.02 & -3.75 & 13.04 & 30 \\
\hline ESO 137-43 & -2.33 & -3.85 & 3.02 & 11 & -4.13 & 7.79 & -8.79 & 8.11 & 63 \\
\hline ESO 165-09 & -6.99 & 7.34 & 2.92 & 75 & -8.09 & 7.72 & 6.34 & 6.52 & 129 \\
\hline ESO 166-04 & -8.69 & 7.40 & 2.92 & 22 & -8.47 & 7.47 & 3.18 & 9.37 & 23 \\
\hline ESO 211-01 & -7.82 & 7.21 & 3.04 & 10 & -5.34 & 8.56 & 3.39 & 9.00 & 29 \\
\hline ESO 261-07 & -9.15 & 2.85 & 2.81 & 80 & -8.65 & 10.59 & 4.23 & 11.24 & 260 \\
\hline ESO 226-06 & -3.79 & -3.59 & 1.92 & 18 & -1.75 & 10.69 & -6.92 & 8.41 & 38 \\
\hline ESO 231-30 & 2.95 & -1.68 & 2.92 & 25 & 3.60 & 5.61 & -8.95 & 7.38 & 152 \\
\hline ESO 260-07 & -5.35 & 7.84 & 3.04 & 24 & -5.36 & 7.27 & 3.65 & 6.67 & 44 \\
\hline ESO 261-03 & -7.77 & 2.88 & 2.80 & 15 & -9.38 & 12.56 & 4.67 & 10.92 & 46 \\
\hline ESO 261-07 & -3.58 & 5.42 & 3.05 & 16 & -6.14 & 11.01 & 5.18 & 8.60 & 43 \\
\hline ESO 275-01 & -0.97 & -1.62 & 2.69 & 26 & -6.11 & 8.41 & -7.34 & 9.07 & 59 \\
\hline ESO 281-24 & 0.42 & -7.61 & 2.80 & 9 & 0.05 & 9.39 & -5.79 & 12.05 & 49 \\
\hline ESO 309-03 & -4.79 & 2.60 & 3.17 & 5 & 0.78 & 6.45 & -1.34 & 22.53 & 27 \\
\hline ESO 311-14 & -5.08 & 5.88 & 2.80 & 20 & -6.62 & 6.12 & 8.95 & 7.77 & 34 \\
\hline ESO 368-14 & -5.89 & 4.64 & 2.57 & 10 & -2.35 & 17.59 & 6.77 & 16.43 & 13 \\
\hline ESO 332-20 & 0.71 & -2.67 & 2.68 & 28 & -3.98 & 8.26 & -3.30 & 5.46 & 88 \\
\hline ESO 311-21 & -7.54 & 2.43 & 2.80 & 8 & -5.82 & 8.74 & 5.91 & 8.99 & 17 \\
\hline ESO $332-22$ & -0.68 & -2.79 & 2.92 & 33 & -1.78 & 6.61 & -3.51 & 7.21 & 93 \\
\hline ESO 329-02 & -7.42 & -4.54 & 2.58 & 10 & -7.01 & 13.40 & -6.26 & 14.04 & 67 \\
\hline ESO 332-08 & -1.69 & -2.53 & 2.92 & 50 & -2.52 & 10.33 & -6.06 & 14.46 & 145 \\
\hline ESO 332-13 & 0.03 & -3.42 & 2.69 & 12 & -1.74 & 13.23 & -6.93 & 13.54 & 31 \\
\hline ESO $337-23$ & -2.28 & -9.08 & 2.67 & 14 & 2.66 & 10.91 & -3.97 & 9.32 & 11 \\
\hline ESO 392-13 & -2.21 & -0.50 & 2.69 & 15 & -1.63 & 9.00 & -3.86 & 9.02 & 49 \\
\hline ESO 393-15 & -3.26 & -2.78 & 2.46 & 24 & -2.17 & 9.64 & -3.83 & 10.20 & 73 \\
\hline ESO 397-01 & 0.39 & -11.95 & 2.93 & 12 & 1.45 & 8.89 & -4.24 & 8.60 & 34 \\
\hline ESO 427-32 & -1.63 & 0.75 & 2.92 & 30 & -1.76 & 10.19 & 3.52 & 9.84 & 66 \\
\hline ESO 429-02 & -0.84 & 1.60 & 2.46 & 20 & -5.14 & 3.32 & -1.09 & 7.18 & 16 \\
\hline ESO 430-14 & -1.57 & 0.77 & 2.92 & 20 & -5.05 & 8.86 & -0.75 & 8.65 & 41 \\
\hline ESO 435-09 & -8.57 & 1.52 & 3.04 & 13 & -9.41 & 11.97 & 0.44 & 8.57 & 52 \\
\hline ESO 447-29 & -3.07 & -3.88 & 2.69 & 13 & -12.50 & 11.85 & -6.15 & 8.08 & 33 \\
\hline ESO 494-09 & -5.08 & 4.69 & 2.80 & 9 & -5.61 & 5.98 & 0.49 & 9.72 & 24 \\
\hline ESO 524-01 & -2.32 & -1.83 & 2.92 & 12 & 0.761 & 8.39 & -0.94 & 8.25 & 54 \\
\hline ESO 522-05 & 2.53 & -2.13 & 2.92 & 8 & -0.68 & 15.58 & 0.37 & 17.79 & 41 \\
\hline
\end{tabular}




\begin{tabular}{|c|c|c|c|c|c|c|c|c|c|}
\hline \multicolumn{5}{|c|}{ MEMBROS } & \multicolumn{5}{|c|}{ Campo } \\
\hline Aglomerado & $\begin{array}{l}\mu \alpha \cos \delta \\
(\mathrm{mas} / \mathrm{yr})\end{array}$ & $\begin{array}{c}\mu \delta \\
(\mathrm{mas} / \mathrm{yr})\end{array}$ & $\sigma$ & $\mathrm{Ne}$ & $\begin{array}{l}\mu \alpha \cos \delta \\
(\mathrm{mas} / \mathrm{y})\end{array}$ & $\sigma_{\mu \alpha \cos \delta}$ & $\begin{array}{c}\mu \delta \\
(\mathrm{mas} / \mathrm{yr})\end{array}$ & $\sigma_{\mu \delta}$ & $\mathrm{Nf}$ \\
\hline ESO 525-08 & 1.43 & 0.29 & 2.68 & 10 & 1.34 & 10.15 & -5.39 & 11.06 & 25 \\
\hline ESO 559-13 & -3.87 & 2.94 & 2.90 & 30 & -3.56 & 9.16 & 1.35 & 10.04 & 144 \\
\hline ESO 561-05 & -5.32 & -0.96 & 2.93 & 11 & -2.55 & 10.24 & 0.68 & 10.78 & 62 \\
\hline ESO 134-12 & -4.53 & -0.83 & 2.57 & 12 & -3.55 & 12.52 & 0.75 & 14.19 & 35 \\
\hline Harvard 13 & -3.26 & -7.60 & 2.69 & 23 & -5.05 & 7.68 & -3.21 & 10.25 & 48 \\
\hline Haffner 14 & -3.80 & 3.04 & 3.04 & 45 & -4.23 & 6.84 & 3.50 & 9.22 & 103 \\
\hline Harvard 16 & -0.65 & -0.94 & 2.92 & 85 & -1.38 & 9.40 & -3.37 & 9.08 & 260 \\
\hline Haffner 22 & -5.16 & 4.62 & 2.92 & 33 & -6.30 & 6.13 & 5.22 & 6.58 & 72 \\
\hline Haffner 3 & -6.37 & -3.94 & 3.05 & 4 & -2.34 & 11.59 & -1.44 & 11.44 & 47 \\
\hline Haffner 4 & 0.17 & -3.94 & 2.93 & 22 & -2.44 & 8.25 & -0.87 & 7.34 & 67 \\
\hline Haffner 5 & -0.37 & -4.62 & 2.67 & 35 & -3.21 & 2.93 & 0.26 & 4.64 & 60 \\
\hline Haffner 7 & -5.21 & 3.67 & 2.69 & 20 & -2.53 & 6.86 & 2.88 & 8.77 & 28 \\
\hline Hogg 13 & -7.87 & 3.05 & 2.92 & 7 & -1.93 & 8.99 & 1.54 & 5.18 & 24 \\
\hline Hogg 19 & -4.17 & -4.53 & 2.92 & 22 & -2.85 & 6.76 & -5.31 & 4.65 & 53 \\
\hline Hogg 20 & -3.39 & -3.87 & 3.05 & 16 & -4.99 & 11.94 & -5.96 & 8.86 & 75 \\
\hline Hogg 2 & -9.87 & 4.97 & 2.92 & 28 & -6.56 & 6.27 & 3.19 & 5.62 & 56 \\
\hline Hogg 3 & -7.09 & 2.56 & 2.67 & 9 & -4.18 & 12.16 & 4.02 & 11.61 & 41 \\
\hline Hogg 7 & -9.63 & 5.89 & 2.81 & 16 & -9.10 & 10.56 & 1.05 & 9.66 & 73 \\
\hline Juchert 12 & -3.12 & -2.47 & 3.04 & 7 & 2.46 & 8.68 & 5.59 & 15.37 & 6 \\
\hline King 17 & -0.74 & -1.40 & 3.17 & 25 & -3.40 & 7.80 & -2.96 & 5.037 & 28 \\
\hline King 23 & -5.27 & -2.48 & 3.05 & 14 & -3.05 & 13.14 & -2.32 & 13.46 & 35 \\
\hline Kronberger 18 & -2.56 & -5.55 & 3.15 & 30 & -3.30 & 7.11 & -7.95 & 2.14 & 30 \\
\hline Kronberger 52 & -0.38 & -3.98 & 2.69 & 10 & -5.48 & 14.29 & -8.25 & 19.38 & 10 \\
\hline Loden 1002 & -5.14 & -4.34 & 2.83 & 30 & -7.60 & 10.04 & -5.57 & 11.07 & 179 \\
\hline Loden 2045 & -5.83 & -3.41 & 2.93 & 60 & -8.64 & 6.99 & -5.46 & 7.55 & 264 \\
\hline Loden 1095 & -7.32 & -1.39 & 2.93 & 36 & -7.54 & 7.51 & -2.22 & 5.53 & 182 \\
\hline Loden 1101 & -8.63 & -3.88 & 2.92 & 50 & -9.07 & 8.75 & -5.47 & 9.58 & 160 \\
\hline Loden 153 & -8.56 & 4.17 & 2.69 & 19 & -11.62 & 12.52 & 2.34 & 12.46 & 21 \\
\hline Loden 2158 & -5.91 & -3.11 & 3.08 & 250 & -6.48 & 11.27 & -4.90 & 11.50 & 1241 \\
\hline Loden 2159 & -5.31 & -2.91 & 3.04 & 170 & -6.78 & 8.00 & -5.14 & 8.44 & 750 \\
\hline Loden 189 & -4.75 & 2.38 & 3.05 & 90 & -7.29 & 8.96 & 0.99 & 8.76 & 599 \\
\hline Loden 1194 & -4.30 & -1.52 & 2.93 & 57 & -6.49 & 10.06 & -3.85 & 9.81 & 263 \\
\hline Loden 213 & -3.00 & 4.03 & 3.05 & 100 & -3.72 & 9.87 & 2.38 & 9.09 & 569 \\
\hline Loden 280 & -4.97 & 1.94 & 3.04 & 13 & -6.06 & 8.53 & 0.15 & 8.89 & 75 \\
\hline Loden 306 & -5.04 & 1.72 & 2.94 & 340 & -5.97 & 10.77 & 1.63 & 10.81 & 1747 \\
\hline Loden 2313 & -3.34 & -2.53 & 2.93 & 120 & -6.22 & 7.73 & -5.61 & 8.37 & 375 \\
\hline Loden 372 & -7.94 & 1.00 & 2.92 & 40 & -9.071 & 9.84 & 1.28 & 6.38 & 95 \\
\hline Loden 1373 & -5.78 & -4.61 & 3.04 & 110 & -7.043 & 10.96 & -4.49 & 10.70 & 589 \\
\hline Loden 1375 & -7.50 & -6.22 & 3.04 & 55 & -8.774 & 7.49 & -5.94 & 7.00 & 291 \\
\hline Loden 1378 & -2.40 & -2.25 & 2.81 & 265 & -4.29 & 10.17 & -3.19 & 9.63 & 717 \\
\hline Loden 402 & -6.53 & 0.66 & 2.93 & 68 & -7.79 & 10.53 & 2.29 & 10.48 & 294 \\
\hline
\end{tabular}




\begin{tabular}{|c|c|c|c|c|c|c|c|c|c|}
\hline \multicolumn{5}{|c|}{ MEMBROS } & \multicolumn{5}{|c|}{ Campo } \\
\hline Aglomerado & $\begin{array}{l}\mu \alpha \cos \delta \\
(\mathrm{mas} / \mathrm{yr})\end{array}$ & $\begin{array}{c}\mu \delta \\
(\mathrm{mas} / \mathrm{yr})\end{array}$ & $\sigma$ & $\mathrm{Ne}$ & $\begin{array}{l}\mu \alpha \cos \delta \\
(\mathrm{mas} / \mathrm{yr})\end{array}$ & $\sigma_{\mu a \cos \delta}$ & $\begin{array}{c}\mu \delta \\
(\operatorname{mas} / y r)\end{array}$ & $\sigma_{\mu \delta}$ & $\mathrm{Nf}$ \\
\hline Loden 1409 & -4.83 & -2.78 & 3.06 & 190 & -6.49 & 11.48 & -4.71 & 11.51 & 1083 \\
\hline Loden 466 & -5.86 & -2.58 & 2.69 & 17 & -8.70 & 9.76 & -1.55 & 9.45 & 66 \\
\hline Loden 467 & -7.69 & -2.54 & 2.92 & 14 & -5.01 & 7.89 & -2.06 & 5.40 & 47 \\
\hline Loden 481 & -4.00 & 1.67 & 2.92 & 360 & -5.49 & 10.37 & 0.21 & 10.26 & 1316 \\
\hline Loden 624 & -3.96 & -1.25 & 3.02 & 830 & -5.51 & 8.00 & -2.73 & 7.55 & 1873 \\
\hline Loden 682 & -6.23 & -1.36 & 2.80 & 300 & -8.19 & 7.90 & -3.26 & 6.25 & 868 \\
\hline Loden 694 & -6.18 & -0.63 & 2.92 & 250 & -7.99 & 8.65 & -2.68 & 7.43 & 540 \\
\hline Loden 757 & -8.86 & -1.82 & 2.92 & 105 & -9.16 & 8.25 & -2.48 & 7.60 & 744 \\
\hline Loden 807 & -5.37 & -2.40 & 3.05 & 140 & -6.09 & 7.74 & -4.07 & 6.00 & 553 \\
\hline Loden 821 & -7.97 & 0.65 & 2.81 & 135 & -8.44 & 11.56 & 0.98 & 11.58 & 517 \\
\hline Loden 848 & -6.63 & -4.34 & 2.92 & 75 & -7.11 & 9.11 & -3.86 & 8.62 & 298 \\
\hline Loden 894 & -6.39 & -3.68 & 3.04 & 33 & -5.20 & 7.78 & -5.03 & 8.05 & 127 \\
\hline Loden 915 & -7.06 & 1.80 & 3.07 & 230 & -8.27 & 8.73 & 1.79 & 7.83 & 1259 \\
\hline Latham 1 & -3.51 & 0.57 & 2.92 & 10 & -6.67 & 16.37 & -4.78 & 14.39 & 197 \\
\hline Loden 28 & -7.97 & 7.02 & 3.04 & 130 & -8.43 & 10.89 & 7.08 & 9.82 & 448 \\
\hline Loden 46 & -7.02 & 3.41 & 2.94 & 750 & -8.47 & 8.70 & 4.52 & 7.69 & 1783 \\
\hline Lynga 11 & -2.97 & -2.97 & 2.69 & 16 & -4.29 & 8.49 & -4.56 & 8.84 & 63 \\
\hline Lynga 12 & -2.56 & -3.71 & 2.81 & 20 & -3.30 & 8.99 & -3.02 & 11.09 & 58 \\
\hline Lynga 15 & -6.17 & -2.59 & 2.92 & 48 & -7.77 & 8.89 & -1.73 & 8.19 & 155 \\
\hline Lynga 3 & -10.74 & -1.23 & 1.72 & 14 & -8.06 & 8.98 & -7.12 & 8.28 & 23 \\
\hline Lynga 5 & -4.44 & -4.24 & 2.80 & 37 & -4.98 & 10.17 & -6.35 & 9.46 & 110 \\
\hline Lynga 9 & -3.12 & -4.66 & 2.69 & 16 & -0.83 & 20.76 & -3.94 & 20.49 & 73 \\
\hline Muzzio 1 & -3.47 & 4.61 & 3.04 & 20 & -9.73 & 10.20 & 7.697 & 9.67 & 53 \\
\hline NGC 956 & -1.00 & -4.27 & 2.69 & 19 & 3.96 & 12.79 & -5.20 & 12.87 & 26 \\
\hline Pismis 12 & -8.97 & 4.07 & 2.80 & 24 & -10.78 & 9.45 & 1.80 & 10.50 & 40 \\
\hline Pismis 21 & -6.48 & -5.00 & 2.57 & 21 & -7.23 & 11.07 & -8.60 & 9.97 & 33 \\
\hline Poole J1855+10.8 & 0.24 & -4.72 & 3.04 & 9 & 1.74 & 5.82 & -4.35 & 9.04 & 45 \\
\hline Ruprecht 100 & -4.21 & -2.84 & 2.92 & 18 & -5.61 & 16.06 & -0.75 & 15.55 & 127 \\
\hline Ruprecht 101 & -6.99 & 0.04 & 2.93 & 70 & -7.12 & 12.03 & 0.79 & 11.33 & 366 \\
\hline Ruprecht 104 & -8.50 & -2.65 & 2.81 & 16 & -9.48 & 11.75 & -6.59 & 13.67 & 45 \\
\hline Ruprecht 105 & -4.11 & -1.63 & 2.80 & 80 & -4.46 & 8.45 & -3.05 & 7.99 & 291 \\
\hline Ruprecht 111 & -6.86 & -5.87 & 2.69 & 35 & -4.29 & 10.12 & -5.08 & 9.31 & 77 \\
\hline Ruprecht 112 & -1.26 & -1.91 & 3.05 & 38 & -1.27 & 7.88 & -3.38 & 4.97 & 199 \\
\hline Ruprecht 117 & -3.34 & -4.78 & 4.04 & 90 & -5.037 & 13.06 & -6.92 & 14.17 & 273 \\
\hline Ruprecht 112 & -5.02 & -6.30 & 2.80 & 50 & -4.596 & 10.29 & -5.24 & 10.27 & 145 \\
\hline Ruprecht 126 & -2.79 & -3.58 & 2.81 & 14 & -2.51 & 7.63 & -4.31 & 10.55 & 45 \\
\hline Ruprecht 131 & 0.17 & -1.11 & 2.92 & 16 & -1.00 & 13.52 & 3.65 & 14.11 & 49 \\
\hline Ruprecht 136 & -3.95 & -3.44 & 2.69 & 13 & 1.53 & 11.18 & -3.11 & 9.65 & 19 \\
\hline Ruprecht 138 & -3.28 & -4.28 & 2.95 & 16 & -2.12 & 12.98 & -3.37 & 13.35 & 108 \\
\hline Ruprecht 140 & -1.00 & -3.25 & 2.93 & 13 & 2.12 & 10.42 & -2.59 & 14.17 & 76 \\
\hline Ruprecht 149 & -2.49 & 2.53 & 3.17 & 12 & -6.44 & 8.92 & 3.08 & 8.90 & 23 \\
\hline
\end{tabular}




\begin{tabular}{|c|c|c|c|c|c|c|c|c|c|}
\hline \multicolumn{5}{|c|}{ MEMBROS } & \multicolumn{5}{|c|}{ Campo } \\
\hline Aglomerado & $\begin{array}{l}\mu \alpha \cos \delta \\
(\mathrm{mas} / \mathrm{yr})\end{array}$ & $\begin{array}{c}\mu \delta \\
(\operatorname{mas} / y r)\end{array}$ & $\sigma$ & $\mathrm{Ne}$ & $\begin{array}{l}\mu \alpha \cos \delta \\
(\operatorname{mas} / y r)\end{array}$ & $\sigma_{\mu \alpha \cos \delta}$ & $\begin{array}{c}\mu \delta \\
(\operatorname{mas} / y r)\end{array}$ & $\sigma_{\mu \delta}$ & $\mathrm{Nf}$ \\
\hline Ruprecht 150 & -1.23 & 0.79 & 1.82 & 9 & -1.48 & 11.10 & 0.60 & 13.22 & 10 \\
\hline Ruprecht 153 & -4.79 & 2.17 & 3.04 & 10 & -7.11 & 11.58 & -1.20 & 8.94 & 9 \\
\hline Ruprecht 155 & -2.35 & 3.81 & 3.04 & 7 & -5.99 & 5.75 & 2.16 & 10.52 & 15 \\
\hline Ruprecht 159 & -5.89 & 4.84 & 2.57 & 6 & -9.81 & 7.51 & 2.98 & 11.83 & 13 \\
\hline Remecht 162 & -4.52 & 4.45 & 2.92 & 17 & -6.46 & 12.52 & 3.82 & 8.65 & 70 \\
\hline Dermecht 163 & -9.25 & 3.98 & 2.92 & 8 & -26.81 & 23.26 & 7.00 & 18.40 & 9 \\
\hline Perrecht 165 & -11.21 & 0.86 & 2.92 & 80 & -13.53 & 8.06 & -0.85 & 4.46 & 276 \\
\hline Rerecht 168 & -1.13 & -3.57 & 2.81 & 11 & 1.40 & 13.49 & -5.11 & 13.99 & 64 \\
\hline Parrecht 170 & -5.45 & -5.22 & 2.92 & 20 & -1.77 & 5.95 & -2.92 & 3.79 & 99 \\
\hline Rernecht 172 & -1.46 & -2.12 & 3.05 & 60 & -1.77 & 7.98 & -2.10 & 9.77 & 374 \\
\hline Ruprecht 173 & 0.67 & -2.11 & 2.92 & 290 & 0.55 & 9.80 & -3.54 & 10.66 & 923 \\
\hline Ruprecht 174 & -1.26 & -2.26 & 3.06 & 34 & -0.17 & 9.27 & -4.90 & 9.76 & 64 \\
\hline Reprecht 175 & 1.20 & -2.34 & 2.92 & 70 & -0.06 & 8.12 & -1.97 & 8.16 & 251 \\
\hline Ruprecht 21 & -4.28 & 3.56 & 3.04 & 40 & -4.17 & 7.00 & 2.15 & 7.09 & 139 \\
\hline Ruprecht 23 & -2.43 & 0.47 & 2.69 & 30 & -0.40 & 4.74 & 1.72 & 5.46 & 29 \\
\hline Ruprecht 24 & 1.03 & 1.02 & 2.13 & 17 & 2.90 & 9.34 & 3.89 & 7.07 & 35 \\
\hline Ruprecht 28 & -4.07 & 4.94 & 3.04 & 10 & -0.62 & 16.80 & -4.55 & 18.14 & 15 \\
\hline Ruprecht 29 & -4.18 & 1.13 & 2.69 & 35 & -2.91 & 4.03 & 2.23 & 8.18 & 38 \\
\hline Ruprecht 30 & -3.62 & 7.21 & 2.80 & 24 & -5.89 & 6.39 & 2.93 & 5.99 & 34 \\
\hline Ruprecht 35 & -1.58 & 4.47 & 3.04 & 6 & -6.65 & 13.18 & -1.73 & 12.66 & 6 \\
\hline Ruprecht 40 & -6.25 & 9.28 & 3.05 & 6 & -6.92 & 9.66 & 6.27 & 7.34 & 18 \\
\hline Ruprecht 42 & -3.68 & 5.37 & 2.69 & 10 & -2.37 & 5.26 & 2.79 & 5.26 & 30 \\
\hline Ruprecht 51 & -4.73 & 1.78 & 2.46 & 21 & -4.08 & 4.21 & 1.43 & 6.46 & 34 \\
\hline Ruprecht 52 & -3.63 & 0.28 & 1.92 & 11 & -0.99 & 9.63 & -4.02 & 9.84 & 10 \\
\hline Ruprecht 58 & -2.74 & 2.14 & 3.05 & 30 & -3.14 & 6.20 & 2.71 & 3.55 & 32 \\
\hline Ruprecht 60 & -1.94 & 7.45 & 2.03 & 12 & -8.31 & 10.09 & 3.74 & 11.44 & 8 \\
\hline Ruprecht 61 & -4.49 & 5.93 & 3.04 & 6 & -4.38 & 9.18 & 3.07 & 15.89 & 25 \\
\hline Ruprecht 63 & -4.07 & 7.21 & 2.81 & 15 & -8.83 & 5.36 & 4.50 & 4.02 & 18 \\
\hline Ruprecht 70 & -0.82 & 5.59 & 2.03 & 21 & -8.11 & 8.69 & 3.71 & 8.94 & 21 \\
\hline Ruprecht 87 & -8.07 & 3.53 & 2.93 & 18 & -9.39 & 9.47 & 4.85 & 12.66 & 51 \\
\hline Ruprecht 95 & -6.61 & 0.58 & 3.05 & 17 & -5.34 & 12.59 & -0.05 & 12.30 & 90 \\
\hline stoff J0507+30.8 & -1.67 & -2.49 & 2.35 & 25 & -0.32 & 3.99 & -2.74 & 6.02 & 25 \\
\hline stiff J0619+18.5 & 1.13 & -2.42 & 3.05 & 139 & 2.17 & 9.75 & -6.35 & 12.67 & 184 \\
\hline Skoff J1942+38.6 & -4.20 & -2.54 & 2.93 & 22 & -3.33 & 12.66 & -2.84 & 14.09 & 162 \\
\hline skiff J0614+12.9 & 1.86 & -5.83 & 2.92 & 20 & -0.58 & 5.72 & -3.50 & 7.36 & 47 \\
\hline Stock 15 & -7.02 & -0.13 & 2.92 & 80 & -7.79 & 9.10 & 0.87 & 8.03 & 257 \\
\hline Teutsch 106 & -6.02 & 5.18 & 2.92 & 20 & -7.59 & 10.39 & 0.79 & 11.88 & 32 \\
\hline Teutsch 12 & -1.14 & -2.72 & 3.17 & 10 & -5.19 & 20.22 & -11.62 & 21.99 & 10 \\
\hline Teutsch 35 & -0.64 & -1.28 & 2.80 & 180 & -0.28 & 10.68 & -1.76 & 12.09 & 403 \\
\hline Teutsch 38 & -6.12 & 3.20 & 2.94 & 209 & -7.20 & 6.71 & 3.77 & 7.32 & 935 \\
\hline Teutsch 39 & -1.20 & -2.75 & 3.04 & 55 & 0.35 & 11.49 & -3.03 & 7.67 & 78 \\
\hline
\end{tabular}




\begin{tabular}{|l|c|c|c|c|c|c|c|c|c|}
\hline \multicolumn{1}{|c|}{ MEMBROS } & \multicolumn{6}{c|}{ Campo } \\
\hline Aglomerado & $\begin{array}{c}\mu \alpha \cos \delta \\
(\mathrm{mas} / \mathrm{yr})\end{array}$ & $\begin{array}{c}\mu \delta \\
(\mathrm{mas} / \mathrm{yr})\end{array}$ & $\sigma$ & Ne & $\begin{array}{c}\mu \alpha \cos \delta \\
(\operatorname{mas} / \mathrm{yr})\end{array}$ & $\sigma_{\mu \alpha \cos \delta}$ & $\begin{array}{c}\mu \delta \\
(\mathrm{mas} / \mathrm{yr})\end{array}$ & $\sigma_{\mu \delta}$ & $\mathrm{Nf}$ \\
\hline Teutsch 66 & -7.20 & 5.37 & 2.69 & 11 & -12.32 & 6.27 & 5.78 & 11.42 & 9 \\
\hline Teutsch 1 & -1.99 & -4.45 & 3.07 & 6 & 0.81 & 25.40 & -8.65 & 22.92 & 6 \\
\hline Trumpler 13 & -8.39 & 3.95 & 3.04 & 15 & -9.06 & 13.74 & 2.38 & 13.46 & 72 \\
\hline Trumpler 20 & -7.39 & -2.86 & 2.69 & 50 & -7.87 & 10.19 & -4.57 & 8.99 & 151 \\
\hline Trumpler 23 & -2.42 & -7.14 & 2.93 & 30 & -4.85 & 13.75 & -5.18 & 13.56 & 185 \\
\hline Trumpler 25 & -0.23 & -1.00 & 2.92 & 57 & 0.70 & 6.96 & -3.57 & 9.48 & 191 \\
\hline Trumpler 34 & -0.20 & -5.91 & 2.69 & 21 & -2.27 & 5.45 & -5.86 & 6.67 & 56 \\
\hline Trumpler 6 & -1.47 & 1.58 & 3.04 & 25 & -0.81 & 8.11 & 4.54 & 9.39 & 60 \\
\hline Waterloo 8 & -1.46 & 2.03 & 3.05 & 17 & -5.08 & 8.14 & 3.29 & 7.53 & 117 \\
\hline
\end{tabular}




\section{Tabela B}

Tabela B: Comparação entre os resultados UCAC2 e resultados extraídos da literatura DAML02. $\mathrm{Ne}$ é o número de estrelas do aglomerado, $\mathrm{Nd}$ o número de estrelas do aglomerado catalogadas no DAML02, $\mu \alpha \cos \delta$ e $\mu \delta$ são as componentes do movimento próprio em milisegundos de arco (maslyr), $\sigma$ é a dispersão do movimento próprio das estrelas do aglomerado (UCAC2), $\sigma_{\mu \alpha \cos \delta}$ e $\sigma_{\mu \delta}$ são a dispersão das componentes de movimento próprio das estrelas de campo (DAML02).

\begin{tabular}{|c|c|c|c|c|c|c|c|c|c|}
\hline \multicolumn{5}{|c|}{ AGLOMERADO } & \multicolumn{5}{|c|}{ DAML02 } \\
\hline Região & $\begin{array}{c}\mu \alpha \cos \delta \\
(\text { mas } / y r)\end{array}$ & $\underset{(\mathrm{mas} / \mathrm{yr})}{\mu \delta}$ & $\sigma$ & $\mathrm{Ne}$ & $\begin{array}{c}\mu \alpha \cos \delta \\
(\mathrm{mas} / \mathrm{yr})\end{array}$ & $\sigma \mu \alpha \cos \delta$ & $\begin{array}{c}\mu \delta \delta \\
(\mathrm{mas} / \mathrm{yr})\end{array}$ & $\sigma \mu \delta$ & Nd \\
\hline Alessi 21 & -0.27 & -1.33 & 2.93 & 281 & -04.00 & 1.30 & +02.80 & 1.30 & 11 \\
\hline Alessi 22 & 0.25 & -3.86 & 3.04 & 300 & -03.60 & 3.40 & -10.50 & 3.40 & 25 \\
\hline Alessi 10 & 0.84 & -3.98 & 3.17 & 40 & +02.32 & 1.08 & -08.57 & 1.08 & 17 \\
\hline Dias 4 & -3.66 & -3.02 & 2.57 & 6 & -04.87 & 0.78 & -04.13 & 0.78 & 19 \\
\hline NGC 2849 & -6.75 & 4.85 & 2.71 & 12 & -07.29 & 1.00 & +04.46 & 1.00 & 12 \\
\hline Loden 821 & -7.98 & 0.65 & 2.81 & 135 & -06.13 & 0.68 & +00.57 & 0.78 & 12 \\
\hline Loden 694 & -6.18 & -0.64 & 2.92 & 250 & -06.23 & 0.56 & +00.90 & 0.62 & 16 \\
\hline Loden 915 & -7.06 & 1.81 & 3.07 & 230 & -08.36 & 0.76 & +01.00 & 0.54 & 11 \\
\hline Loden 402 & -6.53 & 0.67 & 2.93 & 68 & -06.90 & 0.93 & -00.53 & 0.69 & 10 \\
\hline Loden 372 & -7.95 & 1.00 & 2.92 & 40 & -10.55 & 0.82 & -00.81 & 1.07 & 5 \\
\hline Loden 28 & -7.97 & 7.02 & 3.04 & 130 & -08.06 & 0.56 & +04.14 & 0.30 & 7 \\
\hline Loden 682 & -6.23 & -1.37 & 2.80 & 300 & -04.31 & 0.74 & -02.69 & 0.89 & 8 \\
\hline Loden 1194 & -4.31 & -1.53 & 2.93 & 57 & -10.07 & 0.93 & -03.29 & 1.01 & 7 \\
\hline Loden 189 & -4.76 & 2.38 & 3.05 & 90 & -05.73 & 0.74 & +02.91 & 0.84 & 14 \\
\hline Loden 306 & -5.05 & 1.73 & 2.94 & 340 & -06.95 & 0.67 & +00.34 & 0.55 & 14 \\
\hline Ruprecht 105 & -4.12 & -1.63 & 2.80 & 80 & -07.62 & 1.04 & -00.61 & 1.02 & 7 \\
\hline NGC 6738 & -0.23 & -4.38 & 2.93 & 51 & +04.42 & 1.11 & +02.65 & 0.86 & 4 \\
\hline NGC 1996 & -0.33 & -2.47 & 2.46 & 110 & +00.58 & 0.87 & -04.35 & 0.35 & 8 \\
\hline NGC 3036 & -4.82 & 4.63 & 2.80 & 20 & -07.43 & 0.92 & -01.25 & 1.05 & 8 \\
\hline Loden 153 & -8.56 & 4.17 & 2.69 & 19 & -05.85 & 0.68 & +04.13 & 0.75 & 5 \\
\hline NGC 2448 & -2.34 & 2.87 & 3.04 & 205 & -03.80 & 0.44 & +04.69 & 0.38 & 17 \\
\hline Loden 46 & -7.03 & 3.42 & 2.94 & 750 & -14.11 & 0.62 & +02.98 & 0.49 & 18 \\
\hline ESO 166-04 & -8.70 & $\overline{7.41}$ & 2.92 & 22 & -07.14 & 0.69 & +07.91 & 0.77 & 8 \\
\hline ESO 165-09 & -7.00 & 7.35 & 2.92 & 75 & -10.15 & 0.53 & +10.47 & 1.02 & 4 \\
\hline ESO 130-06 & -8.83 & 1.27 & 2.69 & 70 & -10.80 & 0.76 & -00.76 & 0.56 & 12 \\
\hline ESO 128-16 & -3.25 & 7.46 & 2.90 & 70 & -02.57 & 0.63 & +06.10 & 0.52 & 15 \\
\hline Ruprecht 131 & 0.17 & -1.11 & 2.92 & 16 & -00.40 & 1.41 & -02.41 & 1.04 & 4 \\
\hline Collinder 333 & -6.20 & 0.86 & 2.69 & 30 & +00.68 & 1.01 & -04.88 & 1.17 & 4 \\
\hline Collinder 269 & -6.31 & -3.90 & 2.80 & 105 & -07.95 & 0.79 & -03.93 & 0.64 & 10 \\
\hline Collinder 121 & -3.67 & 1.53 & 2.81 & 800 & -03.87 & 0.34 & +04.08 & 0.35 & 19 \\
\hline
\end{tabular}




\begin{tabular}{|c|c|c|c|c|c|c|c|c|c|}
\hline & \multicolumn{4}{|c|}{ AGLOMERADO } & \multicolumn{5}{|c|}{ DAML02 } \\
\hline Região & $\begin{array}{c}\mu \alpha \cos \delta \\
(\mathrm{mas} / \mathrm{yr})\end{array}$ & $\begin{array}{c}\mu \delta \\
(\mathrm{mas} / \mathrm{yr})\end{array}$ & $\bar{\sigma}$ & $\mathrm{Ne}$ & $\begin{array}{r}\mu \alpha \cos \delta \\
(\mathrm{mas} / \mathrm{yr})\end{array}$ & $\sigma \mu \alpha \cos \delta$ & $\begin{array}{c}\mu \delta \\
(\mathrm{mas} / \mathrm{yr})\end{array}$ & $\overline{\sigma \mu \delta}$ & $\mathrm{Ne}$ \\
\hline Alessi-Teutsch 7 & -6.48 & 3.56 & 2.82 & 90 & -08.94 & 0.45 & +05.20 & 0.33 & 17 \\
\hline Alessi-Teutsch 3 & -2.19 & 6.61 & 2.92 & 110 & -03.76 & 0.38 & +08.25 & 0.60 & 12 \\
\hline Alessi-Teutsch 12 & 0.49 & -2.99 & 3.07 & 250 & -03.34 & 0.54 & -07.79 & 0.47 & 17 \\
\hline Alessi-Teutsch 8 & -4.81 & 1.15 & 3.05 & 125 & -04.81 & 0.63 & +03.71 & 0.51 & 20 \\
\hline Alessi-Teutsch 9 & 2.08 & -6.67 & 3.36 & 130 & +11.59 & 0.31 & -09.72 & 0.31 & 26 \\
\hline Alessi 44 & -0.86 & -5.96 & 3.06 & 600 & -01.16 & 0.21 & -02.74 & 0.36 & 14 \\
\hline Alessi 37 & 0.52 & -1.09 & 3.02 & 250 & +01.34 & 0.45 & -02.41 & 0.42 & 9 \\
\hline Alessi 34 & -3.28 & 4.51 & 2.93 & 370 & -05.62 & 0.57 & +06.25 & 0.36 & 12 \\
\hline Alessi 24 & -1.03 & -5.70 & 3.17 & 0 & +01.35 & 0.45 & -08.31 & 0.44 & 46 \\
\hline NGC 2306 & -5.20 & -1.73 & 3.17 & 31 & -00.98 & 0.57 & +01.91 & 0.38 & 9 \\
\hline NGC 6828 & -2.49 & -5.06 & 2.81 & 16 & -01.12 & 1.06 & -02.90 & 0.75 & 4 \\
\hline NGC 2319 & -2.00 & -2.15 & 3.04 & 45 & -00.54 & 0.48 & -01.55 & 0.59 & 7 \\
\hline NGC 2202 & 0.42 & -4.11 & 3.56 & 15 & +00.42 & 0.66 & +01.52 & 0.36 & 5 \\
\hline NGC 6639 & -0.86 & -0.54 & 2.92 & 16 & +02.31 & 0.79 & -01.50 & 0.63 & 7 \\
\hline NGC 2252 & -1.08 & -1.57 & 3.05 & 75 & -01.26 & 0.68 & -04.11 & 0.47 & 6 \\
\hline NGC 5359 & -5.23 & -4.95 & 3.04 & 86 & -05.75 & 0.62 & -01.55 & 0.85 & 6 \\
\hline NGC 5043 & -6.55 & 0.92 & 2.94 & 46 & -10.60 & 0.59 & -00.86 & 0.87 & 13 \\
\hline NGC 1802 & -0.88 & -3.02 & 2.69 & 140 & -00.48 & 0.44 & -08.23 & 0.64 & 7 \\
\hline NGC 1896 & 0.55 & -5.01 & 3.17 & 100 & +00.54 & 0.77 & -08.03 & 0.59 & 6 \\
\hline ESO 332-08 & -1.69 & -2.54 & 2.92 & 50 & +00.05 & 0.30 & -04.10 & 0.37 & 8 \\
\hline Teutsch 38 & -6.13 & 3.20 & 2.94 & 209 & -07.47 & 0.73 & +03.34 & 0.52 & 10 \\
\hline Teutsch 35 & -0.64 & -1.28 & 2.80 & 180 & -01.35 & 0.68 & -02.30 & 0.81 & 17 \\
\hline ESO 559-13 & -3.88 & 2.95 & 2.90 & 30 & -04.15 & 0.81 & +01.91 & 0.81 & 13 \\
\hline Loden 807 & -5.38 & -2.40 & 3.05 & 140 & -06.57 & 0.83 & -02.51 & 0.83 & 17 \\
\hline Loden 481 & -4.00 & 1.67 & 2.92 & 360 & -02.77 & 0.37 & +01.53 & 0.37 & 25 \\
\hline Loden 1409 & -4.83 & -2.78 & 3.06 & 190 & -07.45 & 0.96 & -07.74 & 0.96 & 16 \\
\hline Loden 1378 & -2.41 & -2.25 & 2.81 & 265 & -02.82 & 0.89 & -02.44 & 0.89 & 20 \\
\hline Ruprecht 172 & -1.46 & -2.13 & 3.05 & 60 & -01.27 & 0.66 & -03.13 & 0.66 & 27 \\
\hline Ruprecht 117 & -3.34 & -4.78 & 4.04 & 90 & -03.30 & 0.89 & -05.21 & 0.89 & 17 \\
\hline ESO 427-32 & -1.63 & 0.75 & 2.92 & 30 & -02.09 & 1.18 & +05.03 & 1.18 & 12 \\
\hline NGC 2447 & -5.12 & 3.62 & 2.80 & 90 & -04.85 & 0.33 & +04.47 & 0.33 & 35 \\
\hline ESO 123-26 & -1.48 & 8.14 & 3.05 & 39 & +00.74 & 0.88 & +08.48 & 0.88 & 15 \\
\hline Dolidze 41 & -0.88 & 0.20 & 2.80 & 65 & -02.73 & 0.92 & -04.67 & 0.92 & 12 \\
\hline Ruprecht 175 & 1.21 & -2.34 & 2.92 & 70 & +00.54 & 0.83 & -03.09 & 0.83 & 24 \\
\hline Loden 2313 & -3.35 & -2.53 & 2.93 & 120 & -02.15 & 0.85 & -01.86 & 0.85 & 15 \\
\hline Dolidze 40 & -1.13 & -0.74 & 2.90 & 95 & -02.83 & 0.88 & -03.91 & 0.88 & 12 \\
\hline Dolidze 38 & -1.60 & -2.06 & 3.02 & 130 & -03.44 & 0.97 & -06.19 & 0.97 & 13 \\
\hline Dolidze 36 & -2.19 & -2.32 & 2.92 & 55 & +00.98 & 1.03 & -02.67 & 1.03 & 17 \\
\hline Dolidze 24 & -1.71 & -1.46 & 3.04 & 50 & +00.24 & 0.55 & -03.92 & 0.55 & 19 \\
\hline Collinder 62 & -1.48 & -2.60 & 3.02 & 340 & +00.81 & 1.14 & -06.44 & 1.14 & 18 \\
\hline Collinder 213 & -6.37 & 5.00 & 3.04 & 130 & -04.53 & 0.71 & +03.05 & 0.71 & 12 \\
\hline Collinder 115 & -0.77 & -4.62 & 3.30 & 63 & +00.44 & 0.56 & -03.81 & 0.56 & 17 \\
\hline NGC 6847 & -1.38 & -3.88 & 3.04 & 300 & -02.22 & 0.37 & -04.79 & 0.37 & 63 \\
\hline NGC 6791 & -2.45 & -0.32 & 1.72 & 23 & -03.17 & 1.40 & -07.74 & 1.40 & 15 \\
\hline NGC 5925 & -2.70 & -4.90 & 2.93 & 165 & -04.09 & 0.72 & -03.09 & 0.72 & 21 \\
\hline NGC 5138 & -5.80 & 0.16 & 2.90 & 32 & -03.65 & 0.64 & +01.23 & 0.64 & 20 \\
\hline NGC 1662 & -0.74 & -1.87 & 2.03 & 70 & -01.93 & 0.28 & -02.10 & 0.28 & 18 \\
\hline
\end{tabular}




\section{Tabela C}

Tabela C: Valores dos parâmetros fundamentais (distâncias, excessos de cor nas bandas do infravermelho e idades) para o nosso ajuste e os valores publicados por Pauzen e Netopil [32].

\begin{tabular}{|c|c|c|c|c|c|c|}
\hline \multirow{2}{*}{ NOME } & \multicolumn{3}{|c|}{ AJUSTE } & \multicolumn{3}{|c|}{ PAUZEN } \\
\hline & Distância & $\mathrm{E}(\mathrm{J}-\mathrm{H})$ & $\log t$ & Distância & $\mathrm{E}(\mathrm{J}-\mathrm{H})$ & $\log t$ \\
\hline NGC6834 & 1750 & 0,20 & 7,90 & 2147 & 0,23 & 7,81 \\
\hline NGC1647 & 480 & 0,10 & 8,40 & 492 & 0,11 & 8,11 \\
\hline Trumpler10 & 400 & $-0,03$ & 9,40 & 422 & 0,02 & 7,57 \\
\hline IC2395 & 530 & 0,03 & 8,90 & 792 & 0,03 & 7,18 \\
\hline Bochum10 & 1700 & 0,07 & 7,40 & 2472 & 0,10 & 6,90 \\
\hline Stock14 & 1600 & 0,07 & 7,15 & 2439 & 0,07 & 7,00 \\
\hline NGC5662 & 480 & 0,07 & 8,50 & 684 & 0,09 & 7,89 \\
\hline NGC5822 & 600 & 0,16 & 8,90 & 787 & 0,04 & 8,96 \\
\hline NGC6025 & 410 & 0,04 & 8,50 & 725 & 0,05 & 7,87 \\
\hline NGC6087 & 780 & 0,04 & 8,25 & 893 & 0,06 & 7,89 \\
\hline NGC6067 & 1350 & 0,13 & 7,70 & 1676 & 0,11 & 7,97 \\
\hline NGC6250 & 600 & 0,10 & 7,70 & 936 & 0,11 & 7,34 \\
\hline NGC6405 & 320 & 0,04 & 8,80 & 473 & 0,04 & 7,32 \\
\hline Collinder 394 & 550 & 0,07 & 8,90 & 648 & 0,08 & 7,87 \\
\hline NGC4756 & 380 & 0,12 & 9,20 & 415 & 0,06 & 8,83 \\
\hline NGC6871 & 1350 & 0,11 & 7,10 & 1675 & 0,14 & 6,95 \\
\hline NGC2682 & 1000 & 0,25 & 8,50 & 820 & 0,02 & 8,98 \\
\hline NGC1245 & 2380 & 0,15 & 8,80 & 2593 & 0,08 & 9,03 \\
\hline NGC2527 & 570 & 0,03 & 9.40 & 581 & 0,02 & 8,79 \\
\hline NGC2477 & 1037 & 0,09 & 8,75 & 1227 & 0,08 & 8,94 \\
\hline
\end{tabular}




\section{Tabela D}

Tabela D: Tabela dos 11 aglomerados conhecidos utilizados no cálculo de parâmetros fundamentais. São listadas algumas das estrelas extraídas do SIMBAD utilizadas nos cálculos e suas respectivas probabilidades de pertinência, bem como os ST de cada uma delas. Também são apresentados os resultados de saída do programa Stax; E(J-H) e d são o excesso de cor, obtidos a partir do cálculo no infravermelho; e $\mathrm{E}(\mathrm{J}-\mathrm{H})$ e d também obtidos a partir do cálculo no visível. Por fim os resultados para $\mathrm{E}(\mathrm{J}-\mathrm{H})$ e $\mathrm{d}$ obtidos no ajuste junto aos diagramas cor-magnitude.

\begin{tabular}{|c|c|c|c|c|c|c|c|c|c|}
\hline \multirow{3}{*}{ Aglo } & \multicolumn{2}{|l|}{ SIMBAD } & \multicolumn{4}{|c|}{ Programa } & \multicolumn{3}{|c|}{ Ajuste CMD } \\
\hline & & & Infrave & melho & Vis & & & 3 & Cक्य \\
\hline & Nome Estrela. & S.T. & $\mathrm{E}(\mathrm{J}-\mathrm{H})$ & dist & $\mathrm{E}(\mathrm{J}-\mathrm{H})$ & dist & $\mathrm{E}(\mathrm{J}-\mathrm{H})$ & dist & $\log t$ \\
\hline NGC 1907 & HD 281143 - 59\% & F2 & 0,07 & 186 & 0,02 & 176 & 0,13 & 1450 & 8,45 \\
\hline NGC 2099 & $\begin{array}{l}\mathrm{Cl}^{*} \text { NGC } 2099 \text { WEST B - } 94 \% \\
\mathrm{Cl}^{*} \text { NGC } 2099 \text { WEST I }-94 \% \\
\mathrm{Cl}^{*} \text { NGC } 2099 \text { WEST H-93\% } \\
\mathrm{Cl}^{*} \text { NGC } 2099 \text { WEST G- } 94 \% \\
\mathrm{Cl}^{*} \text { NGC } 2099 \text { BRON } 330-94 \% \\
\mathrm{BD}+321112-93 \% \\
\mathrm{Cl}^{*} \text { NGC } 2099 \text { BRON } 416-94 \% \\
\mathrm{HD} 248521-94 \%\end{array}$ & $\begin{array}{c}\text { A2 } \\
\text { A0 } \\
\text { A0 } \\
\text { A0 } \\
\text { G5 } \\
\text { K1III } \\
\text { F0 } \\
\text { F5 }\end{array}$ & 0,09 & 431 & 0,13 & 474 & 0,09 & 1250 & 8,65 \\
\hline NGC 2168 & $\begin{array}{c}\text { HD } 41940-88 \% \\
\text { HD } 252260-87 \% \\
\text { HD } 252176-88 \% \\
\text { HD } 252199-91 \% \\
\text { HD } 252198-89 \% \\
\text { HD } 252197-89 \%\end{array}$ & $\begin{array}{l}\text { B8 } \\
\text { K5 } \\
\text { A0 } \\
\text { A0 } \\
\text { B9 } \\
\text { K2 }\end{array}$ & 0,04 & 762 & 0,05 & 578 & 0,07 & 600 & 7,95 \\
\hline NGC 6793 & $\begin{array}{c}\text { HD 344329-70\% } \\
\text { HD 344326-76\% } \\
\text { HD 344330- } 76 \%\end{array}$ & $\begin{array}{l}\text { A0 } \\
\text { A0 } \\
\text { A3 }\end{array}$ & 0,08 & 651 & 0,07 & 717 & 0,17 & 1000 & 8,35 \\
\hline NGC 6834 & $* *$ WSI $13 \mathrm{AB}-80 \%$ & F0Iab & & & 0,17 & & 0,2 & 1750 & 7,65 \\
\hline & & & & & & & & & \\
\hline
\end{tabular}




\begin{tabular}{|c|c|c|c|c|c|c|c|c|c|}
\hline \multirow{3}{*}{ Aglo } & \multicolumn{2}{|l|}{ SIMBAD } & \multicolumn{4}{|c|}{ Programa } & \multicolumn{3}{|c|}{ A juste CMD } \\
\hline & & & Infrav & rmelho & Visíve & & & & \\
\hline & Nome Estrela & S.T. & $\mathrm{E}(\mathrm{J}-\mathrm{H}$ & dist & $\mathrm{E}(\mathrm{J}-\mathrm{H})$ & dist & $\mathrm{E}(\mathrm{J}-\mathrm{H})$ & dist & $\log t$ \\
\hline NGC 6940 & $\begin{array}{l}\mathrm{Cl} * \text { NGC } 6940 \text { HOAG } 10-79 \% \\
\text { HD 334751-78\% } \\
\text { Cl* NGC } 6940 \text { GN } 28-69 \%\end{array}$ & $\begin{array}{l}\text { F0 } \\
\text { A0 } \\
\text { F0 }\end{array}$ & 0,12 & 414 & 0,07 & 522 & 0,07 & 900 & 8,55 \\
\hline Trumpler 21 & $\begin{array}{l}\text { HD } 117513-80 \% \\
\text { CD-62 733-75\% }\end{array}$ & $\begin{array}{l}\text { B3 } \\
\text { B2 }\end{array}$ & 0,075 & 1789 & 0,075 & 1712 & 0,075 & 1100 & 7,75 \\
\hline NGC 1647 & $\begin{array}{l}\text { HD 285995-75\% } \\
\text { HD 285994-75\% } \\
\text { BD+18 708-79\% } \\
\text { HD 285996-75\% } \\
\text { HD 30170--79\% } \\
\text { HD 286004-77\% } \\
\text { BD+18 711-77\% } \\
\text { HD 286006-70\% } \\
\text { HD 284839-75\% } \\
\text { HD 284843-73\% } \\
\text { HD 286003-77\% } \\
\text { HD 285993-63\% } \\
\text { HD 285997-73\% } \\
\text { HD 286016-70\% } \\
\text { HD 284841-73\% } \\
\text { HD 284844-77\% } \\
\text { HD 286014-70\% } \\
\text { HD 285992-76\% } \\
\text { HD 284840-73\% } \\
\text { HD 286007-75\% } \\
\text { HD 285998-76\% } \\
\text { HD 30263 -76\% } \\
\text { HD 286008-78\% } \\
\text { HD 286017-71\% } \\
\text { HD 284735 -76\% }\end{array}$ & $\begin{array}{l}\text { B8 } \\
\text { B8 } \\
\text { A0 } \\
\text { A0 } \\
\text { B8 } \\
\text { A0 } \\
\text { A2 } \\
\text { A0 } \\
\text { A0 } \\
\text { B8 } \\
\text { B5 } \\
\text { B8 } \\
\text { A0 } \\
\text { B8 } \\
\text { B8 } \\
\text { A0 } \\
\text { B8 } \\
\text { B9 } \\
\text { A5 } \\
09 \\
\text { A0 } \\
\text { A0 } \\
\text { B9 } \\
\text { A0 }\end{array}$ & 0,10 & 655 & 0,09 & 621 & 0,1 & 480 & 8,4 \\
\hline NGC 1662 & $\begin{array}{c}\text { BD+10 639B-89\% } \\
\text { HD 30470-92\% } \\
\text { HD 30492-91\% } \\
\text { HD 287148-90\% } \\
\text { HD 287147-91\% } \\
\text { HD 287146-91\% } \\
\text { HD 287151-81\% } \\
\text { HD 287150-91\% } \\
\text { HD 287149-91\% } \\
\text { HD 287158-91\% } \\
\text { HD 287157-89\% }\end{array}$ & $\begin{array}{l}\text { G5 } \\
\text { A2 } \\
\text { A0 } \\
\text { K0 } \\
\text { A3 } \\
\text { A0 } \\
\text { A2 } \\
\text { A2 } \\
\text { A3 } \\
\text { A7 } \\
\text { A2 }\end{array}$ & 342 & 0,09 & 304 & 0.10 & 0,16 & 600 & 8,7 \\
\hline
\end{tabular}




\begin{tabular}{|c|c|c|c|c|c|c|c|c|c|}
\hline \multirow{3}{*}{ Aglo } & \multicolumn{2}{|l|}{ SIMBAD } & \multicolumn{4}{|c|}{ Programa } & \multicolumn{3}{|c|}{ Ajuste CMD } \\
\hline & \multirow[b]{2}{*}{ Nome Estrela } & \multirow[b]{2}{*}{ S.T. } & \multicolumn{2}{|c|}{ Infravermelho } & \multicolumn{2}{|c|}{ Visível } & & & \\
\hline & & & $\mathrm{E}(\mathrm{J}-\mathrm{H})$ & dist & $\mathrm{E}(\mathrm{J}-\mathrm{H})$ & dist & $\mathrm{E}(\mathrm{J}-\mathrm{H})$ & dist & $\log t$ \\
\hline NGC 1750 & $\begin{array}{l}\text { BD+23 821-89\% } \\
\text { HD 285125-89\% } \\
\text { HD 285126-89\% } \\
\text { V* RT Tau-90\% } \\
\text { HD 285127-90\% } \\
\text { AG }+23470-88 \% \\
\text { HD 285111-89\% } \\
\text { HD 285128-89\% }\end{array}$ & $\begin{array}{l}\text { A0 } \\
\text { A0 } \\
\text { B9 } \\
\text { B8 } \\
\text { A2 } \\
\text { B9 } \\
\text { A2 } \\
\text { B5 }\end{array}$ & 0,05 & 743 & 0,08 & 604 & 0,08 & 750 & 8,8 \\
\hline NGC 2447 & CPD-23 2776-80\% & A0 & 471 & 0,08 & 498 & 0,07 & 0,05 & 1000 & 9 \\
\hline
\end{tabular}




\section{Tabela E}

Tabela E: Apresentamos o nome das estrelas e seus ST's extraídos do SIMBAD bem como suas probabilidades de pertinência fornecidas através do Método descrito na seção 2.2 para o cálculo de movimentos próprios. As estrelas foram usadas nos cálculos dos valores iniciais de distâncias e excessos de cor feitos pelo programa Stax. Os parâmetros fundamentais estão dispostos nas colunas, sendo que exibimos distância, excesso de cor nas bandas do infravermelho $\mathrm{E}(\mathrm{J}-\mathrm{H})$. Nas colunas temos resultados de saída do programa, obtidos a partir da nossa análise, vide seção 3.3 e por fim os valores catalogados no DAML02. Os valores originalmente obtidos do DAML02 para excessos de cor estão nas bandas do visível, aqui utilizamos a relação de conversão: $\frac{E(J-H)}{E(J-K)}=0,309$. Os símbolos “*” indicam os aglomerados para os quais obtivemos como resultados de saída do programa apenas o excesso de cor $\mathrm{E}(\mathrm{J}-\mathrm{H})$ calculado nas bandas do visível ou $\mathrm{E}(\mathrm{J}-\mathrm{H})$ calculado nas bandas do infravermelho, respectivamente para Alessi-Teutsch 8 e Loden 28, conforme foi explanado na seção 3.3.

\begin{tabular}{|c|c|c|c|c|c|}
\hline \multirow{2}{*}{ Nome } & \multicolumn{2}{|l|}{ SIMBAD } & \multicolumn{3}{|c|}{ Valores dos parâmetros } \\
\hline & Nome Estrela-Probabilidade & ST & Programa & Ajuste & DAML02 \\
\hline Alessi 21 & $\begin{array}{c}\text { HD 55240-55\% } \\
\text { BD-08 1785-55\% }\end{array}$ & $\begin{array}{l}\text { A0 } \\
\text { A0 }\end{array}$ & $\begin{array}{c}647 \\
-0.01\end{array}$ & $\begin{array}{c}900 \\
0.04 \\
8.9\end{array}$ & $\begin{array}{c}500 \\
0.02 \\
7.5\end{array}$ \\
\hline Alessi 34 & $\begin{array}{c}\text { CD-50 3101-68\% } \\
\text { HD 66845-64\% } \\
\text { CD-50 3128-51\% } \\
\text { HD 66917-66\% } \\
\text { CD-50 3094-67\% } \\
\text { HD 66257-67\% }\end{array}$ & $\begin{array}{l}\text { A0 } \\
\text { B8 } \\
\text { A2 } \\
\text { B7 } \\
\text { G5 } \\
\text { B7 }\end{array}$ & $\begin{array}{l}810 \\
0.01\end{array}$ & $\begin{array}{l}900 \\
0.04 \\
8.05\end{array}$ & $\begin{array}{c}1100 \\
0.05 \\
7.9\end{array}$ \\
\hline
\end{tabular}




\begin{tabular}{|c|c|c|c|c|c|}
\hline \multirow{2}{*}{ Nome } & \multicolumn{2}{|l|}{ SIMBAD } & \multicolumn{3}{|c|}{ Valores dos parâmetros } \\
\hline & Nome Estrela--Probabilidade & ST & Programa & Ajuste & DAML02 \\
\hline Alessi 37 & $\begin{array}{l}\text { HD 215954- 76\% } \\
\text { HD 215856-74\% } \\
\text { HD 216037-78\% } \\
\text { HD 216002-77\% } \\
\text { HD 215926-78\% } \\
\text { HD 216015-77\% }\end{array}$ & $\begin{array}{l}\text { A0 } \\
\text { B9 } \\
\text { A0 } \\
\text { A2 } \\
\text { A0 } \\
\text { A0 }\end{array}$ & $\begin{array}{c}464 \\
-0.03\end{array}$ & $\begin{array}{r}480 \\
0.01 \\
8.5\end{array}$ & $\begin{array}{l}600 \\
0.05 \\
8.48\end{array}$ \\
\hline $\begin{array}{l}\text { Alessi- } \\
\text { Teustch } 3\end{array}$ & $\begin{array}{l}\text { CD-52 2165-51\% } \\
\text { CD-52 2161-51\% }\end{array}$ & $\begin{array}{l}\text { A0 } \\
\text { A0 }\end{array}$ & $\begin{array}{l}647 \\
0.05\end{array}$ & $\begin{array}{c}850 \\
0.07 \\
8.9\end{array}$ & $\begin{array}{c}800 \\
0.03 \\
8.6\end{array}$ \\
\hline $\begin{array}{c}\text { Alessi-Teutsch } \\
8 \\
\end{array}$ & HD $104617-65 \%$ & B9III & $-0.33^{*}$ & $\begin{array}{l}750 \\
0.1 \\
8.0\end{array}$ & $\begin{array}{l}650 \\
0.03 \\
8.65\end{array}$ \\
\hline $\begin{array}{c}\text { Collinder } \\
121\end{array}$ & $\begin{array}{c}\text { HD } 51285-64 \% \\
\text { CD-24 4637-69\% } \\
\text { HD } 51112-53 \% \\
\text { HD 51763-61\% } \\
\text { HD 51088-57\% } \\
\text { HD 51038-69\% }\end{array}$ & $\begin{array}{c}\text { B2 } \\
\text { F8 } \\
\text { A8 } \\
\text { A1 } \\
\text { B9 } \\
\text { B3 }\end{array}$ & $\begin{array}{l}779 \\
0.03\end{array}$ & $\begin{array}{c}1000 \\
0.04 \\
8.1\end{array}$ & $\begin{array}{c}1100 \\
0.01 \\
7.1\end{array}$ \\
\hline Eso $332-08$ & $\begin{array}{l}\text { HD 322282-80\% } \\
\text { HD 322279-82\% } \\
\text { HD 322275-82\% } \\
\text { HD 322277-75\% } \\
\text { HD 322278-82\% } \\
\text { HD 322274-75\% } \\
\text { NSV 20842-80\% }\end{array}$ & $\begin{array}{l}\text { B0 } \\
\text { B9 } \\
\text { B5 } \\
\text { B8 } \\
\text { B8 } \\
\text { B5 } \\
\text { B3 }\end{array}$ & $\begin{array}{l}954 \\
0.06\end{array}$ & $\begin{array}{c}350 \\
0.02 \\
9.1\end{array}$ & $\begin{array}{l}1200 \\
0.06 \\
8.17\end{array}$ \\
\hline
\end{tabular}




\begin{tabular}{|c|c|c|c|c|c|}
\hline \multirow{2}{*}{ Nome } & \multicolumn{2}{|l|}{ SIMBAD } & \multicolumn{3}{|c|}{ Valores dos parâmetros } \\
\hline & Nome Estrela--Probabilidade & ST & Programa & Ajuste & DAML02 \\
\hline Loden 28 & TYC8611-14601-63\% & A9 & $0.08^{*}$ & $\begin{array}{l}1400 \\
0.06 \\
9.35\end{array}$ & $\begin{array}{c}3950 \\
0.19 \\
7.3\end{array}$ \\
\hline Loden 46 & $\begin{array}{l}\text { HD 299106-56\% } \\
\text { HD 299110-61\% }\end{array}$ & $\begin{array}{l}\text { A0 } \\
\text { A0 }\end{array}$ & $\begin{array}{l}985 \\
0.03\end{array}$ & $\begin{array}{c}1000 \\
0.06 \\
8.9\end{array}$ & $\begin{array}{c}540 \\
0.04 \\
9.0\end{array}$ \\
\hline Loden 189 & $\begin{array}{l}\text { HD } 94063-74 \% \\
\text { HD } 301078-63 \% \\
\text { HD 301077-55\% } \\
V^{*} \text { AA Vel-44\% }\end{array}$ & $\begin{array}{l}\text { B6 } \\
\text { A0 } \\
\text { A0 } \\
\text { B5 }\end{array}$ & $\begin{array}{l}1225 \\
0.02\end{array}$ & $\begin{array}{c}600 \\
0.02 \\
8.7\end{array}$ & $\begin{array}{l}720 \\
0.04 \\
8.64\end{array}$ \\
\hline $\begin{array}{l}\text { Loden } \\
306\end{array}$ & $\begin{array}{c}\text { HD } 306001-55 \% \\
\text { HD } 306002-53 \% \\
\text { HD } 96264-54 \% \\
\text { CCDM J1 1049-6103B-56\% }\end{array}$ & $\begin{array}{l}\text { B5 } \\
\text { B5 } \\
\text { O9 } \\
\text { B4 }\end{array}$ & $\begin{array}{l}1521 \\
0.054\end{array}$ & $\begin{array}{l}1200 \\
0.03 \\
8.45\end{array}$ & $\begin{array}{l}2000 \\
0.07 \\
6.76\end{array}$ \\
\hline $\begin{array}{l}\text { Loden } \\
481\end{array}$ & $\begin{array}{l}\text { HD } 307231-72 \% \\
\text { HD } 307232-70 \% \\
\text { HD } 307230-67 \% \\
\text { HD } 102938-53 \%\end{array}$ & $\begin{array}{l}\text { A0 } \\
\text { A2 } \\
\text { A0 } \\
\text { A6 }\end{array}$ & $\begin{array}{l}831 \\
0.04\end{array}$ & $\begin{array}{c}1300 \\
-0.01 \\
8.7\end{array}$ & $\begin{array}{c}1520 \\
0.07 \\
8.2\end{array}$ \\
\hline $\begin{array}{l}\text { Loden } \\
694\end{array}$ & $\begin{array}{l}\text { HD } 312107-54 \% \\
\text { HD } 312117-67 \% \\
\text { HD } 312104-71 \% \\
\text { HD } 311941-71 \%\end{array}$ & $\begin{array}{l}\text { B5 } \\
\text { B8 } \\
\text { B5 } \\
\text { A0 }\end{array}$ & $\begin{array}{l}1298 \\
0.08\end{array}$ & $\begin{array}{c}750 \\
-0.03 \\
8.9\end{array}$ & $\begin{array}{l}1700 \\
0.07 \\
7.38\end{array}$ \\
\hline Loden 807 & HD $116314-55 \%$ & B5 & $\begin{array}{l}1418 \\
0.03\end{array}$ & $\begin{array}{c}600 \\
-0.015 \\
9.3\end{array}$ & $\begin{array}{c}925 \\
0.06 \\
8.3\end{array}$ \\
\hline & & & & & \\
\hline
\end{tabular}




\begin{tabular}{|c|c|c|c|c|c|}
\hline \multirow{2}{*}{ Nome } & \multicolumn{2}{|l|}{ SIMBAD } & \multicolumn{3}{|c|}{ Valores dos parâmetros } \\
\hline & Nome Estrela--Probabilidade & ST & Programa & Ajuste & DAML02 \\
\hline $\begin{array}{l}\text { Loden } \\
821\end{array}$ & HD $116300-57 \%$ & A0 & $\begin{array}{l}819 \\
0.03\end{array}$ & $\begin{array}{c}750 \\
0.08 \\
8.3\end{array}$ & $\begin{array}{c}2800 \\
0.15 \\
7.3\end{array}$ \\
\hline Loden 915 & $\begin{array}{c}\text { TYC 8675-58\% } \\
\text { TYC 8675- 57\% } \\
\text { GSC 08675-57\% } \\
\text { TYC 8675- 74\% } \\
\text { TYC 8675-65\% } \\
\text { TYC 8675-66\% } \\
\text { HD } 117723-60 \% \\
\text { TYC 8674-68\% } \\
\text { GSC 08675-03136-65\% } \\
\text { CD-58 5126-13 3-63\% } \\
\text { TYC 8675- 3182-1 -69\% } \\
\text { TYC 8675- 1712-1 -61\% } \\
\text { GSC 08675-00150-70\% } \\
\text { GSC 08675-03107-72\% } \\
\text { TYC } 8674-1485-1-57 \%\end{array}$ & $\begin{array}{l}\text { A0 } \\
\text { A4 } \\
\text { A4 } \\
\text { B5 } \\
\text { A5 } \\
\text { A0 } \\
\text { B9 } \\
\text { G0 } \\
\text { B9 } \\
\text { A2 } \\
\text { A5 } \\
\text { A2 } \\
\text { A2 } \\
\text { A2 } \\
\text { A3 }\end{array}$ & $\begin{array}{l}1324 \\
0.06\end{array}$ & $\begin{array}{c}950 \\
0.17 \\
9.1\end{array}$ & $\begin{array}{l}500 \\
0.05 \\
8.44\end{array}$ \\
\hline $\begin{array}{l}\text { Loden } \\
2313\end{array}$ & $\begin{array}{l}\text { CD-52 6906-60\% } \\
\text { CD-52 6903-55\% }\end{array}$ & $\begin{array}{l}\text { B8 } \\
\text { B8 }\end{array}$ & $\begin{array}{l}736 \\
0.14\end{array}$ & $\begin{array}{l}700 \\
0.1 \\
8.6\end{array}$ & $\begin{array}{c}1410 \\
0.015 \\
8.75\end{array}$ \\
\hline $\begin{array}{l}\text { NGC } \\
1896\end{array}$ & HD $243395-56 \%$ & A2 & $\begin{array}{l}440 \\
0.03\end{array}$ & $\begin{array}{c}1300 \\
0.13 \\
8.0\end{array}$ & $\begin{array}{c}820 \\
0.05 \\
8.8\end{array}$ \\
\hline NGC 2448 & CD-24 5872-65\% & B8 & $\begin{array}{l}1080 \\
0.033\end{array}$ & $\begin{array}{c}1000 \\
0.01 \\
8.3\end{array}$ & $\begin{array}{c}1040 \\
0.01 \\
7.2\end{array}$ \\
\hline $\begin{array}{l}\text { NGC } \\
2252\end{array}$ & TYC $154-713-1-61 \%$ & B0 & $\begin{array}{l}3300 \\
0.21\end{array}$ & $\begin{array}{l}900 \\
0.1 \\
8.9\end{array}$ & $\begin{array}{l}900 \\
0.03 \\
8.85\end{array}$ \\
\hline & & & & & \\
\hline
\end{tabular}




\begin{tabular}{|c|c|c|c|c|c|}
\hline \multirow{5}{*}{ Teutsch 35 } & HD 185015-77\% & A0 & & & \\
& CCDM J19363+3540AB -84\% & A0 & & & \\
& BD+35 3703C-81\% & A0 & 610 & 720 & 700 \\
& HD 185173-81\% & A0 & -0.03 & 0.01 & 0 \\
& BD+35 3701-81\% & A0 & & 8.25 & 8.37 \\
& HD $185172-84 \%$ & A0 & & & \\
& BD+35 3704-82\% & A0 & & & \\
& HD 184906-81\% & A0 & & & \\
& BD+35 3693-83\% & K5 & & & \\
\hline
\end{tabular}




\section{Tabela F}

Tabela F: Tabela dos 11 aglomerados inéditos cuja solução de parâmetros fundamentais foi obtida neste trabalho. Fornecemos posições (J2000), onde $\alpha$ é dada em (hh mm ss - hora minuto segundo) e ( $\delta$ - grau minuto segundo). São listadas algumas das estrelas extraídas do SIMBAD utilizadas nos cálculos de valores preliminares e suas respectivas probabilidades de pertinência, bem como os ST de cada uma delas. $\mathrm{E}(\mathrm{J}-\mathrm{H})$, d e logt denotam os resultados obtidos no ajuste junto aos diagramas cor-magnitude para os parâmetros fundamentais.

\begin{tabular}{|c|c|c|c|c|c|c|c|}
\hline \multirow{2}{*}{ Nome } & \multicolumn{2}{|c|}{ Posição } & \multicolumn{2}{|l|}{ SIMBAD } & \multicolumn{3}{|c|}{ Ajuste } \\
\hline & $\alpha$ & $\delta$ & Estrela - \% & ST & Distância & $\mathrm{E}(\mathrm{J}-\mathrm{H})$ & $\log t(t)$ \\
\hline Collinder 416 & 201135 & +263204 & $\begin{array}{l}\text { HD } 339670-71 \% \\
\text { BD+26 3824- } 75 \% \\
\text { V*V382 Vul - 74\% }\end{array}$ & $\begin{array}{l}\text { A0 } \\
\text { K5 } \\
\text { B8 }\end{array}$ & 700 & 0.08 & 9.25 \\
\hline Skiff J0619+18.5 & 061922 & +183230 & $\mathrm{AG}+18582-86 \%$ & $\mathrm{~A} 3$ & 1000 & 0.06 & 9.20 \\
\hline NGC 2260 & 063803 & -012824 & $\begin{array}{c}\text { HD 292016-59\% } \\
\text { HD 292011-75\% } \\
\text { HD 47316-66\% } \\
\text { HD 292009-72\% }\end{array}$ & $\begin{array}{l}\mathrm{A} 2 \\
\mathrm{~A} 0 \\
\mathrm{~A} 3 \\
\mathrm{~A} 0\end{array}$ & 750 & 0.10 & 8.50 \\
\hline Loden 373 & 144148 & -625200 & CPD-62 4234-55\% & B2 & 750 & 0.16 & 8.90 \\
\hline Loden 757 & 131224 & -651800 & $\mathrm{HD} 114517-71 \%$ & B8III & 600 & 0.03 & 8.20 \\
\hline Collinder 62 & 052253 & +410451 & $\begin{array}{l}\text { HD 278049-79\% } \\
\text { HD 278056-77\% } \\
\text { HD 278055-69\% } \\
\text { HD 278047-73\% } \\
\text { HD 278057-69\% }\end{array}$ & $\begin{array}{l}\mathrm{K} 2 \\
\mathrm{~B} 5 \\
\mathrm{~A} 0 \\
\mathrm{~B} 8 \\
\mathrm{~A} 2\end{array}$ & 750 & 0.06 & 8.70 \\
\hline
\end{tabular}


105

\begin{tabular}{|c|c|c|c|c|c|c|c|}
\hline \multirow{2}{*}{ Nome } & \multicolumn{2}{|c|}{ Posição } & \multicolumn{2}{|l|}{ SIMBAD } & \multicolumn{3}{|c|}{ Ajuste } \\
\hline & $\alpha$ & $\delta$ & Estrela - \% & ST & Distância & $E(J-H)$ & $\log t(t)$ \\
\hline Antalova 3 & 173034 & -321230 & $\begin{array}{l}\text { HD } 317721-84 \% \\
\text { HD } 317724-84 \%\end{array}$ & $\begin{array}{l}\mathrm{A} 2 \\
\mathrm{~A} 2\end{array}$ & 500 & 0.08 & 8.80 \\
\hline Alessi 62 & 185549 & +213449 & $\begin{array}{l}\text { HD } 343445-75 \% \\
\text { HD } 343429-74 \% \\
\text { HD } 343448-75 \%\end{array}$ & $\begin{array}{l}\text { G0 } \\
\text { K0 } \\
\text { A3 }\end{array}$ & 530 & 0.12 & 9.30 \\
\hline NGC 6847 & 195637 & +301248 & $\begin{array}{c}\text { HD 333101-80\% } \\
\text { HD 333121-79\% } \\
\text { HD 333107-79\% } \\
\text { V* V1356 Cyg-76\% } \\
\text { BD+30 3817-79\% } \\
\text { HD 332952-79\% } \\
\text { AN 201.1928-58\% }\end{array}$ & $\begin{array}{l}\text { K0 } \\
\text { A2 } \\
\text { A0 } \\
\text { B0 } \\
\text { M2 } \\
\text { B0 } \\
\text { G5 }\end{array}$ & 1000 & 0.05 & 8.65 \\
\hline Loden 624 & 122622 & -614300 & $\begin{array}{l}\text { HD108132-63\% } \\
\text { HD107944-64\% }\end{array}$ & $\begin{array}{l}\text { B7III } \\
\text { F2II }\end{array}$ & 850 & 0.08 & 8.20 \\
\hline
\end{tabular}




\section{Referências}

[1] DIAS, W.S., et. al., New catalogue of optically visible open clusters and candidates, Astronomy and Astrophysics, v. 389, n. 3, p. 871-873, julho 2002.

[2] DIAS, W.S., et. al., Proper motions of open clusters within $1 \mathrm{kpc}$ based on the TYCHO2 Catalogue, Astronomy and Astrophysics, v. 376, n. 2, p. 441-447, setembro 2001.

[3] DIAS, W.S., et. al., Proper motions of open clusters based on the TYCHO2 Catalogue - II. Clusters farther than $1 \mathrm{kpc}$, Astronomy and Astrophysics, v.388, n. 1, p. 168-171, junho 2002.

[4] DIAS, W.S., ASSAFIN, M., FLÓRIO, V., et. al., Proper motion determination of open clusters based on the UCAC2 catalogue, Astronomy and Astrophysics, v. 446 , n. 3 , p. $949-953$, fevereiro 2006 .

[5] ALESSI, B.S., et. al., Searching for unknown open clusters in the Tycho-2 catalog , Astronomy and Astrophysics, v. 410, n. 2, p. 565-U4, novembro 2003.

[6] VASILEVSKIS, S., RACH, R.A., Relative proper Motions of stars in the region of the open cluster NGC-6940, Astronomical Journal, v. 62, n. 6, p. 175-182, 1957.

[7] SANDERS, W.L., Improved method for computing membership probabilities in open clusters, Astronomy and Astrophysics, v. 14, n. 2, p. 226, 1971.

[8] ZHAO, J.L., HE, Y.P., An improved method for membership determination of stellar clusters with proper motions with different accuracies, Astronomy and Astrophysics, v. 237, n. 1, p. 54-60, outubro 1990.

[9] ZACHARIAS, N., et. al., The second US naval observatory CCD astrograph catalog (UCAC2), Astronomical Journal, v. 127, n. 5, p. 3043-3059, maio 2004. 
[10] SKRUTSKIE, M., SCHNEIDER, S. E., STIENING, R., et. al., In the impact of large scale IR sky surveys, ed. F. Garzon et. al., netherlands: Kluver, 1997.

[11] SARTORI, M.J., LEPINE, J.R.D., DIAS, W.S., Formation scenarios for the young stellar associations between galactic longitudes $I=280$ degrees-360 degrees, Astronomy and Astrophysics, v. 404, n. 3, p. 913-926, junho 2003.

[12] BERGHOFER, T.W., BREITSCHERDT, D., The origin of the young stellar population in the solar neighborhood - A link to the formation of the Local Bubble?, Astronomy and Astrophysics, v. 390, n. 1, p. 299-306, julho 2002.

[13] LÉPINE, J. R., ACHAROVA, Y., MISHUROV, I. A., Boletim da Sociedade Astronômica Brasileira, v. 23, n. 1, P. 28, 2003.

[14] DIAS, W.S., LÉPINE, J.R.D., Direct determination of the spiral pattern rotation speed of the galaxy, Astronomical Journal, v. 629, n. 2, p. 825-831, Part 1, agosto 2005 .

[15] ALTER, G., RUPRECHT, J., VANÝSEK, V., Review of Publications - Catalogue of Star Clusters and Associations, Journal of the Royal Astronomical Society of Canada, v. 52, p. 189, agosto 1958.

[16] LYNGA, G., Computer Based Catalogue of Open Cluster Data, Milky Way Galaxy : IAU Symposium, p.143, 1985.

[17] MERMILLIOD, J. C., In information and on-line data in astronomy, ed. D. Egret and M. A. Albrecht (Dordrecht:Kluwer), p. 127, 1995.

[18] DEMARQUE, P., IAU Symposium, n. 85, ed. J. E., Hesser, p. 281, 1980.

[19] LEEUWEN, V. F., IAU Symposium, p.579, 1985.

[20] VASILEVSKIS, S., KLEMOLA, A., PRESTON, G., Relative proper motions of stars in the region of the open cluster NGC-6633, Astronomical Journal, v. 63, n. 9 , p. $387-395,1958$.

[21] SLOVAK, M. H., Maximum-likelihood method for determination of membership in open clusters, Astronomical Journal, v. 82, n. 10, p. 818-823, 1977.

[22] DIAS, W. S., Aglomerados Abertos : Determinação de Movimentos Próprios e membership, 2000, 130p. Dissertação (Mestrado)em Astronomia - Instituto Astronômico e Geofísico, Universidade de São Paulo, São Paulo, 2000. 
[23] VASIlEVSKIS, S., SANDERS, W., L. and Van AlTEnA., W., F., Membership of the open cluster IC 1805, Astronomical Journal, v. 70, p. 806, 1965.

[24] HOG, E., FABRICIUS, C., MAKAROV, V.V., et. al., The Tycho-2 catalogue of the 2.5 million brightest stars, Astronomy and Astrophysics, v. 355, n. 2, p. L27-L30, março 2000.

[25] BESSEL, M. S., BRETT, M., JHKLM Photometry: Standards systems, passbands and intrinsic colors, Publications of the Astronomical Society of the Pacific, v.100, p. 1134-1151, setembro 1988.

[26] KOORNNEEF, J., 1.Near infrared photometry. 2. Intrinsic colors and the absolute calibration from onde to five micron, Astronomy and Astrophysics, v. 128 , n. 1, p. 84-93, 1983.

[27] MIHALAS, D., and BINNEY, J., Galactic Astronomy, 2 ed. ; San Francisco: Freeman, 1981.

[28] SCHLEGEL, D.J., FINKBEINER, D.P., DAVIS, M., Maps of dust infrared emission for use in estimation of reddening and cosmic microwave background radiation foregrounds, Astrophysical Journal, v. 500, n. 2, p. 525-553, Part 1, junho 1998.

[29] NEUGEBAUER, G. and LEIGHTON, R., B., Two-micron sky survey: A preliminary catalogue, tmss book, 1969.

[30] KHARCHENKO, N.V., et. al., Astrophysical parameters of Galactic open clusters, Astronomy and Astrophysics, v. 438 n. 3, p. 1163-1173, agosto 2005.

[31] MacNamara, B., J., et. al., Astronomy and Astrophysics, v. 27, p. 117, 1977.

[32] BICA, E., SANTIAGO, B.X., DUTRA, C.M., et. al., Dissolving star cluster candidates, Astronomy and Astrophysics, v. 366, n. 3, p. 827-833, fevereiro 2001.

[33] BONATTO, C., BICA, E., GIRARDI, L., Theoretical isochrones compared to 2MASS observations: Open clusters at nearly solar metallicity, Astronomy and Astrophysics, v. 415, n. 2, p. 571-576, fevereiro 2004.

[34] PAUZEN, M., NETOPIL, M., On the current status of open clusters parameters, Mon. Not. R. Astron. Soc. 000, 000000, julho 2006. 